\title{
THREE-DIMENSIONAL SELF-ASSEMBLED NANOCARBON FOR THERANOSTICS APPLICATIONS
}

\author{
by \\ A K M Rezaul Haque Chowdhury \\ Master of Engineering, Ryerson University (2014) \\ Master of Business Administration, University of Dhaka (1998) \\ Bachelor of Science, Bangladesh University of Engineering and Technology (1995)
}

\author{
A dissertation \\ presented to Ryerson University \\ in partial fulfilment of the \\ requirements for the degree of \\ Doctor of Philosophy \\ in the program of \\ Aerospace Engineering
}

Toronto, Ontario, Canada, 2018

(C)A K M Rezaul Haque Chowdhury, 2018 


\section{AUTHOR'S DECLARATION FOR ELECTRONIC SUBMISSION OF A DISSERTATION}

I hereby declare that I am the sole author of this dissertation. This is a true copy of the dissertation, including any required final revisions, as accepted by my examiners.

I authorize Ryerson University to lend this dissertation to other institutions or individuals for the purpose of scholarly research.

I further authorize Ryerson University to reproduce this dissertation by photocopying or by other means, in total or in part, at the request of other institutions or individuals for the purpose of scholarly research.

I understand that my dissertation may be made electronically available to the public. 


\title{
Three-dimensional self-assembled nanocarbon for theranostics applications
}

\author{
(C) A K M Rezaul Haque Chowdhury, \\ Doctor of Philosophy, Aerospace Engineering, Ryerson University, Toronto (2018)
}

\begin{abstract}
Carbon nanomaterials have been explored for biomedical applications such as scaffolds in tissue engineering, drug delivery carriers, cancer diagnostics and biological imaging. Due to their possible cytotoxicity and biological inertness, they need biological or chemical functionalization to attain biomedical applications. Current research trends are for the synthesis of biocompatible and self-functionalized nanocarbon with prospective application in therapy and diagnosis. The main objectives of this thesis are to synthesize 3D self-functionalized biocompatible nanocarbon for therapeutic and diagnostic applications. The synthesis of the unique three-dimensional carbon nanostructures has been done with ultrashort femtosecond laser processing mechanism, a versatile yet precise technique for nanoscale material generation.

First study deals with the synthesis of 3D nanocarbon network and its biocompatibility assessment. Quantitative and qualitative studies of the fibroblast cell response to this nano-network are performed. The findings from the in-vitro study indicate that the platform possesses excellent biocompatibility and promote cell adhesion and subsequent cell proliferation.

In next study, the synthesized nanocarbon network (CNRN) platform that possesses a variation in C-C and C-O bond architecture showed dual functionality i. e. cytophilic to fibroblasts but cytotoxic to HeLa cells. Two distict opposite responses like tissue generation for fibroblasts and
\end{abstract}


apoptosis like function for HeLa was observed after 48-hour of culture. The results have potentials for therapeutic appliations.

Third study focuses on the diagnostic applications of the nanocarbon. A unique non-plasmonic SERS based bio-sensing platform using 3D nanocarbon is introduced for in-vitro detection and differentiation of HeLa and fibroblast cells. Time based Raman spectroscopy of these cells seeded on nanocarbon revealed chemical fingerprints of intracellular components like DNA/RNA, protein and lipids. Their spectroscopic differences guide differentiation of each cell.

Finally, we have synthesized $\mathrm{N}$-enriched nanocarbon probe through nitrogen incorporationassisted ionization and demonstrate label free SERS based detection of transient variation of cell chemistry and thereby cancer cell diagnosis with $\mathrm{N}$-enriched 3D nanocarbon probe. The results suggested that the SERS functionality not only reveal the chemical fingerprint of the intracellular components (e. g. protein, DNA, RNA etc.) within a cell but also guide detection of cancerous HeLa cells.

The results obtained in this thesis point out multifunctional viability of biocompatible selffunctionalized nanocarbons for therapy and diagnosis. 


\section{Acknowledgements}

I would like to express my sincere appreciation for my supervisors Dr. Bo Tan and Dr. Krishnan Venkatakrishnan for allowing me the opportunity to pursue my academic interests through this research project. Their advice and boundless support has assisted me in becoming a better researcher and has given me the skills to be successful in future actions.

I would like ro thank my committee members Dr. Hekmat Alighanbari, Dr. Puren Ouyang and Dr. Siyuan He for their valuable guidance and careful advice to shape the direction of my research project and refining my thesis.

I would like to express my deepest gratitude to my wife and two kids for their sacrifices and support throughout my entire academic career. I would also like to thank my mother and my three sisters for their constant encouragement from far away. Without them I could not have done this.

I would like to thank all my lab-mates specially Sivaprasad Chinnakkannu Vijayakumar, Chandramouli Chinnakkannu Vijayakumar, Jeff Powell, Meysam Keshavarz and Pooja Thakur who have helped me with my research over the last four years.

Finally, I thank the Almighty for setting the stage for my success. 


\section{Dedication}

To my wife Zebunnessa, my children Rayna and Zuhayer and my mom Rokshana Khanum. 


\section{Table of Contents}

Author's declaration for electronic submission

Abstract

Acknowledgements $\quad$ V

Dedication vi vi vis vis

List of Tables $\quad$ xii

List of Figures $\quad$ xiii

List of Abbreviations $\quad$ xix

$\begin{array}{ll}\text { Chapter } 1 & 1\end{array}$

$\begin{array}{ll}\text { Introduction } & 1\end{array}$

1.1 Carbon nanostructures for biomedical applications 1

1.2 Carbon nanostructures in tissue engineering and therapeutic applications 1

1.3 Carbon nanostructures in bio-sensing and diagnostics applications 3

1.4 Prevailing synthesis trends for carbon nanostructures/nanomaterials 4

1.5 Summary 6

$\begin{array}{llr}1.6 & \text { Research objectives } & 6\end{array}$

$\begin{array}{lll}1.7 & \text { Organization of the thesis } & 7\end{array}$

$\begin{array}{lr}\text { Chapter } 2 & 9\end{array}$

Biofunctionalized 3-D Carbon Nano-Network Platform for Enhanced Fibroblast Cell $\begin{array}{lr}\text { Adhesion } & 9\end{array}$

$\begin{array}{ll}2.1 \text { Introduction } & 10\end{array}$

2.2 Experimental Method 13

2.2.1 Carbon nano-network platform fabrication 13

2.2.2 Morphological and physicochemical characterization of 3-D network 14

$\begin{array}{ll}\text { 2.2.3 Cell Culture } & 15\end{array}$

2.2.4 NIH-3T3 mouse fibroblast cells-material interaction 15

2.2.5 Fluorescent staining of cells 15

$\begin{array}{ll}\text { 2.2.6 Statistics } & 16\end{array}$

2.3 Results 16

2.3.1 Synthesis of 3-D nanofibrous network on Graphite substrate 16 
2.3.2 Cell interaction with carbon nano-network

$\begin{array}{ll}2.4 \text { Discussions } & 30\end{array}$

2.5 Summary 34

$\begin{array}{ll}\text { Chapter } 3 & 35\end{array}$

Fibroblast-Cytophilic and HeLa-Cytotoxic Dual Function Carbon Nanoribbon Network Platform 35

3.1 Introduction 36

3.2 Experimental Method 39

3.2.1. Bioactive CNRN Platform Fabrication. 39

3.2.2. Morphological and Physicochemical Characterization of the CNRN Platform. $\quad 40$

3.2.3. Cell Culture. 41

3.2.3.1. NIH-3T3 Mouse Fibroblast Cells and Cervical Cancer Cells (HeLa) seeding on CNRN Platform. 41

3.2.3.2. Fluorescent Staining of Cells. 42

3.2.4. Statistics 42

3.3 Results $\quad 42$

3.3.1. Fabrication and Characterization of the CNRN Platform 42

3.3.2 Fibroblasts and HeLa cell response to the bio active carbon nanoribbon network 49

3.3.2.1 Time dependent cell fate $\quad 49$

3.3.2.2 Effect of varying dosage of the CNRN and C-O concentration 50

3.3.2.3. Cell Morphology 51

3.3.2.4 Characteristics of Growing Cells $\quad 56$

3.3.2.5 CNRN Uptake $\quad 59$

3.4 Discussions $\quad 62$

3.4.1 CNRN Formation $\quad 62$

3.4.2 Cytophilic Behavior $\quad 63$

3.4.3 Cytotoxic Behavior $\quad 65$

$\begin{array}{ll}3.5 \text { Summary } & 65\end{array}$

$\begin{array}{ll}\text { Chapter } 4 & 68\end{array}$

SERS Active 3D Interconnected Nanocarbon Web Towards Non-plasmonic In-vitro $\begin{array}{lc}\text { Sensing of HeLa and Fibroblast Cells. } & 68\end{array}$ 
$\begin{array}{ll}4.1 \text { Introduction } & 69\end{array}$

4.2 Experimental Method $\quad 75$

4.2.1 Interconnected nanocarbon web platform fabrication 75

4.2.2 Morphological characterization of INW platform 76

4.2.3 Physicochemical characterization of Nanocarbon platform 76

$\begin{array}{ll}\text { 4.2.4 Cell culture } & 77\end{array}$

4.2.4.1. Fibroblast and HeLa Cells seeding on nanocarbon Platform 77

4.2.4.2 Fluorescence microscopy of Cells 78

$\begin{array}{ll}4.2 .5 \text { Statistics } & 78\end{array}$

$\begin{array}{ll}4.3 \text { Results } & 79\end{array}$

4.3.1 Fabrication of 3D interconnected nanocarbon web(INW) platform 79

4.3.2 Morphology and physicochemical characterization of 3D INW platform $\quad 81$

4.3.3 SERS efficiency of 3D INW platform and EF calculation 82

4.3.3.1Raman Enhancement factor(EF) calculation 84

4.3.4 INW cluster uptake $\quad 88$

4.3.5 SERS Raman spectra of the live fibroblast and Hela cells 89

4.3.5.1 SERS spectra of live fibroblast cells 90

4.3.5.2 SERS spectra of live HeLa cells 90

4.3.5.3 Identification of Fibroblast and HeLa cells from live SERS Raman spectra 92

4.3.5.4 SERS effect on live Fibroblast and HeLa cells 92

4.3.6 SERS Raman spectra of the immobilized fibroblast and Hela cells 94

4.3.7 SERS Raman spectra of the single fibroblast and Hela cell (immobilized) $\quad 100$

$\begin{array}{ll}\text { 4.4 Discussions } & 102\end{array}$

4.4.1 3D interconnected nanocarbon web formation 102

4.4.2 SERS active nanocarbon based detection of fibroblast and HeLa 103

$\begin{array}{ll}\text { 4.4.3 Cell health } & 104\end{array}$

$\begin{array}{ll}\text { 4.5 Summary } & 105\end{array}$

$\begin{array}{ll}\text { Chapter } 5 & 107\end{array}$

SERS active Nitrogen-Enriched 3-D Carbon Nanoprobe for Cancerous HeLa Cells

$\begin{array}{ll}\text { Diagnosis. } & 107\end{array}$

$\begin{array}{ll}5.1 \text { Introduction } & 108\end{array}$ 
5.2. Materials and Method

5.2.1 Nitrogen-enriched 3D carbon nanoprobe sensor fabrication

5.2.2 Morphological characterization

5.2.3 Physicochemical characterization

5.2.4 Cell culture

5.2.4.1. Fibroblast and HeLa Cells seeding on N-CNPs sensor Platform

114

5.2.4.2. Fluorescence microscopy of Cells.

5.2.5 Statistics

5.3. Results and Discussion

5.3.1 N-CNP sensor platform formation

5.3.2 Morphology and physicochemical characterization of N-CNP sensor platform

5.3.3 SERS efficiency of N-CNP sensor platform and EF calculation

5.3.4 Favorable micro environment by N-CNP sensor platform

5.3.4.1 Favorable micro environment

5.3.4.2 N-CNP unit structure uptake

5.3.5 SERS Raman spectra of the live Hela and fibroblast cells

5.3.5.1 SERS spectra of live fibroblast cells

5.3.5.2 SERS spectra of live HeLa cells

5.3.5.3 SERS Raman spectra of the immobilized fibroblast and Hela cells

\section{Chapter 6}

Summary and Future Research

6.1 Summary

6.2 Future Research 
References 


\section{List of Tables}

Page \#

Table 2-1. Crystallite size (La) calculation using Equation 1 and the Id and Ig intensities from

Micro-Raman spectra of nano-network platforms developed at different fluences.

Table 3-1: Calculation of CNRN Index of the created CNRN platform for change in ionization

energy from low to high 
Figure 1-1: Schematic diagram depicting the controlled cell growth on fibronectin-carbon nanotube (FN-CNT) hybrid nanostructures (courtesy [13])

Figure 1-2: A schematic diagram of HeLa cell with $\mathrm{C}-\mathrm{Co}-\mathrm{NPs}$ apoptosis process under RF excitation. (courtesy [19])

Figure 1-3: Schematic design of graphene oxide based biosensor. (courtesy [22])

Figure1-4: A schematic synthesis apparatus for classical laser ablation used for production of carbon nanotube. (courtesy [29])

Figure 2-1: Schematic of the laser ionization of graphite substrate and the synthesis of nanonetwork platform and its corresponding SEM micrographs.

Figure 2-2: The SEM and corresponding FE-SEM micrographs of (A) native graphite substrate and the nanonetwork platforms created at (B) low (C) medium, and (D) high laser fluence.

Figure 2-3: (A) A comparison of Micro-Raman spectra of native graphite and the nano-network platform synthesized at different laser fluences, (B) the relationships between crystallite size La and ID/IG ratio, and (C) the influence of the laser fluences on the crystallite size La. The ID/IG ratio is recorded from the Micro-Raman spectra, and it is used to calculate crystallite size La using Equation 1.

Figure 2-4: XPS spectra of (A) native graphite substrate and (B) the nano-network platform; (C) XPS quantitative analysis of major elements on both untreated graphite substrate and the nano-network platform created at high fluence.

Figure 2-5: (A) FSEM-EDX elemental mapping of the created nano-network platforms at different laser fluences and their corresponding oxygen $(O)$ and Carbon $(C)$ spectra; (B) Comparison of XRD patterns of native graphite substrate and the nano-network platform indicating the presence of standard crystalline nano-graphite pattern as of native graphite except the peak at $2 \theta \approx 32$ which is associated to crystalline carbon (C-00-046-0943).

Figure 2-6: Schematic of native graphite and the nano-network platforms fabricated at different laser fluences and the corresponding SEM micrographs of the substrates before and after fibroblast response after $24 \mathrm{~h}$. (Graphics of the Petri dish and the cells were adapted from Servier Medical Art - creativecommons.org). 
Figure 2-7: SEM micrographs of fibroblasts adhered on (A) native graphite and (B-D) the nanonetwork platforms created at low, medium and high fluences, respectively; corresponding fluorescence microscopy images of $(\mathrm{E}-\mathrm{G})$ nano-network platforms created at low, medium and high fluence, respectively after $24 \mathrm{~h}$ of culture; and SEM micrographs of fibroblasts cultured on (H-J) low, medium and high fluence mediated nanonetwork, respectively, after $48 \mathrm{~h}$.

Figure 2-8: Quantitative analysis of the number of NIH 3 T3 fibroblasts adhered on the untreated graphite substrate and the nano-network platforms created at different fluences.

Figure 2-9: SEM micrographs of the fibroblast cells adhered on low, medium and high fluence mediated nanonetwork platforms, respectively, after (A-C) $24 \mathrm{~h}$ and (D-F) $48 \mathrm{~h}$ of culture.

Figure 2-10: Fluorescence microscopy images and quantitative analysis of the surface area of the nucleus of fibroblasts attached on the platforms created at (A) low (B) medium, and (C) high laser fluence.

Figure 3-1: Graphical abstract showing bio activation of carbon nanoribbon network with multiphoton ionization and time dependent fibroblast and HeLa cell response on the created carbon nanoribbon network.

Figure 3-2: Schematic illustration showing the single-step synthesis of the CNRN platform. Change in ionization energy precisely modulated the physical morphology and the $\mathrm{C}-\mathrm{O}$ bond percentage of the CNRN platform.

Figure 3-3: (A) CNRN index of the synthesized CNRN platform with different $\mathrm{C}-\mathrm{O}$ percentage phases and density numbers at low, medium, and high ionization energies. (B) Nanoribbon width frequency histogram and nanovoid diameter frequency histogram of the created nanonetwork.

Figure 3-4: (A) XPS spectra of the carbon nanoribbon network having different C-O percentage phases with the (B) corresponding quantitative analysis of the major elements in the created carbon nanoribbon network; (C) deconvoluted O1s XPS spectra and (D) deconvoluted C1s XPS spectra for CNRN platform; (E) Micro-Raman spectra of the graphite substrate and the graphite substrate with the carbon nanoribbon network having different $\mathrm{C}-\mathrm{O}$ percentage phases and the (F) influence of different $\mathrm{C}-\mathrm{O}$ percentage phases on the $\mathrm{I}_{\mathrm{D}} / \mathrm{I}_{\mathrm{G}}$ ratio. 
Figure 3-5: Fibroblasts and HeLa cell responses on (A) native graphite substrate and the (B) bio active carbon nanoribbon network platform. The graphite substrate has no cell response for both fibroblasts and HeLa cell after $24 \mathrm{~h}$. The created CNRN platform has positive cell response with a cell selective cytophilic/cytotoxic response on fibroblasts and HeLa cells after $24 \mathrm{~h}$ and $48 \mathrm{~h}$, respectively.

Figure 3-6: fibroblast and HeLa cell adhesion and proliferation after $24 \mathrm{~h}$ and $48 \mathrm{~h}$, on varying dosage of the CNRN and C-O concentration; Experiments were repeated with $n=3$. Statistical significance is shown with $* \mathrm{p}<0.05, * * \mathrm{p}<0.01$.

Figure 3-7: SEM micrographs of the fibroblasts and HeLa cells adhered to the (A) $20 \% \mathrm{C}-\mathrm{O}$ phase, (B) $22 \% \mathrm{C}-\mathrm{O}$ phase, and (C) $23 \% \mathrm{C}-\mathrm{O}$ phase, along with the corresponding FM images after $24 \mathrm{~h}$ of culture.

Figure 3-8: Comparison of the morphology of individual fibroblasts and HeLa cells adhered on the various $\mathrm{C}-\mathrm{O}$ percentage phase $\mathrm{CNRN}$ platforms; SEM micrograph of (A) fibroblasts [A(i, ii)] and HeLa cells [A(iii, iv)] on the $20 \% \mathrm{C}-\mathrm{O}$ phase, (B) fibroblasts [B(i, ii)] and HeLa cells [B(iii, iv)] on the $22 \% \mathrm{C}-\mathrm{O}$ phase, and (C) fibroblasts [C(i,ii)] and HeLa cells [C(iii,iv)] on the $23 \% \mathrm{C}-\mathrm{O}$ phase after 24 and $48 \mathrm{~h}$ of culture, respectively.

Figure 3-9: FM images of the HeLa cell and fibroblast cytoskeletons stained in green and the nucleus stained in blue attached to the CNRN with (A) $20 \% \mathrm{C}-\mathrm{O}$ dominant phase, (B) $22 \%$ C-O dominant phase, and (C) $23 \% \mathrm{C}-\mathrm{O}$ dominant phase.

Figure 3-10: Quantitative analysis of the fibroblasts and HeLa cells on the CNRN with different percentage $\mathrm{C}-\mathrm{C}$ dominant phases. The error bars show the standard error of the mean; experiments were repeated with $\mathrm{n}=3$. Statistical significance is shown with $* \mathrm{p}<0.05$ and $* * \mathrm{p}$ $<0.01$.

Figure 3-11: Effect of CNRN cluster endocytosis of the HeLa cell and fibroblast cytoskeletons attached to the CNRN platform.

Figure 3-12: EDX mapping images of the HeLa and fibroblast cytoskeletons attached to the CNRN with (A) $20 \% \mathrm{C}-\mathrm{O}$, (B) $22 \% \mathrm{C}-\mathrm{O}$, and (C) $23 \% \mathrm{C}-\mathrm{O}$ dominant phases. 
Figure 4-1: Overall graphical abstract showing the formation of SERS active 3D nanocarbon web, SERS detection with CV and R6G dyes and subsequent SERS based in vitro sensing of live cells.

Figure 4-2: Schematic illustration depicting the one-step synthesis of the 3D interconnected nanocarbon web platform. The physical morphology and the $\mathrm{C}-\mathrm{O}$ bond ratio of the nanocarbon structure accurately modulated with the change in ionization energy.

Figure 4-3: (A) Synthesized 3D interconnected nanocarbon web platform with (B) different nanocarbon width and $\mathrm{C}-\mathrm{O}$ concentrations phases at low, medium, and high ionization energies. (C) Nanocarbon width frequency histogram (D) XPS spectra of the nanocarbon with different $\mathrm{C}-\mathrm{O}$ concentrations phases along with $(\mathrm{E})$ corresponding quantitative assessment of the major elements.

Figure 4-4: (A) Raman Spectra of the graphite substrate and the INW structure containing graphite substrate with different $\mathrm{C}-\mathrm{O}$ concentrations (B) ISERS/I $\mathrm{I}_{\text {substrate }}$ ratio at different nanocarbon width for $\mathrm{D}$ and $\mathrm{G}$ band

Figure 4-5: (A) Raman spectral enhancement with CV dye (B) Raman EF values with CV dye for $\mathrm{D}$ and $\mathrm{G}$ band at different nanocarbon width (C) Raman spectral enhancement with R6G dye (D) Raman EF values with R6G dye for D and $\mathrm{G}$ band at different nanocarbon width.

Figure 4-6: Schematic of the charge-transfer mechanism principle.

Figure 4-7: (A) INW cluster uptake by the fibroblast and HeLa cytoskeletons attached to the nanocarbon platform and (B) EDX mapping images of the Fibroblasts and HeLa cytoskeletons attached to the nanocarbon platform showed internalized INW clusters.

Figure 4-8: SERS Raman spectra of live fibroblast and HeLa cells seeded on different C-O concentrations INW platform for 24 hours incubation period with 6 hours intervals.

Figure 4-9: (A-D) Gradual changes in SERS Raman spectra for live Hela and Fibroblast cells on high C-O concentration INW platform acted as basis for cell differentiation (E) SERS based Raman signal enhancement of intracellular components of HeLa cells (F) SERS based Raman signal enhancement of intracellular components of Fibroblast cells

Figure 4-10: (A)Variation in different intracellular components peaks of immobilized fibroblast cells based on SERS Raman spectra obtained from 24 and 48 hours samples containing different C-O concentrations INW platforms along with SEM and fluorescence micrograph and (B) Variation in different intracellular components peaks of immobilized fibroblast cells based on 
SERS Raman spectra obtained from 24 and 48 hours samples containing different C-O concentrations INW platforms along with SEM and fluorescence micrograph.

Figure 4-11: Quantitative analysis of different types of fibroblast and HeLa cells on nanocarbon platform with varying C-O concentrations. The error bars show the standard error of the mean; experiments were repeated with $\mathrm{n}=3$. Statistical significance is shown with $* \mathrm{p}<0.05$ and $* * \mathrm{p}$ $<0.01$.

Figure 4-12: (A)SERS Raman spectra of immobilized single fibroblast cell along with confocal image for 24-hour sample containing different C-O concentrations nanocarbon platforms and (B) SERS Raman spectra of immobilized single HeLa cell along with confocal image for 24-hour sample containing different C-O concentrations nanocarbon platforms.

Figure 5-1: Graphical abstract showing formation of 3D interconnected Nitrogen enriched carbon nanoprobe sensor platform, SERS activation with CV dye and subsequent SERS based early detection of HeLa and Fibroblast cells

Figure 5-2: A) Schematic illustration depicting the one-step femtosecond laser synthesis of the $\mathrm{N}$-enriched 3D interconnected carbon nanoprobe B) FESEM and TEM images showing physical morphology of the created $\mathrm{N}$-enriched 3D interconnected carbon nanoprobe.

Figure 5-3: (A) FSEM-EDX elemental mapping of the created N-CNPs sensor platform (B) Nenriched carbon nanoprobe width frequency histogram; (C) XPS spectra of the N-CNP structures with different $\mathrm{C}-\mathrm{N}$ percentage phases along with the extended $\mathrm{C} 1 \mathrm{~s}, \mathrm{~N} 1 \mathrm{~s}$ and $\mathrm{O} 1 \mathrm{~s}$ spectra and the (D) corresponding quantitative analysis of the major elements in the created N-CNPs sensor platform.

Figure 5-4: (A) SERS Raman enhancement of N-CNP sensor platform with CV dye (B) Raman EF values with CV dye for D and G band at different N-CNP width (C) Schematic of the chargetransfer mechanism principle for SERS enhancement.

Figure 5-5: (A) Quantitative analysis of the fibroblasts and HeLa cells adhered on the N-CNP sensor platform with different percentage $\mathrm{C}-\mathrm{N}$ dominant phases indicating favorable microenvironment for SERS. The error bars show the standard error of the mean; experiments were repeated with $\mathrm{n}=3$. Statistical significance is shown with $* \mathrm{p}<0.05$ and $* * \mathrm{p}<0.01(\mathrm{~B}) \mathrm{N}$ CNP sensor platform showed higher microenvironment favorability for cell adhesion (C) Schematics showing N-CNP unit structure uptake by the fibroblast and HeLa cytoskeletons 
attached to the N-CNP sensor platform and (D) The favorability ratio showed an increasing trend with the increment of $\mathrm{C}-\mathrm{N}$ enrichment in the N-CNP sensor platform.

Figure 5-6: SERS Raman spectra of (A) live fibroblast cell and (B)HeLa cells seeded on different $\mathrm{C}-\mathrm{N}$ percentage N-CNP sensor platform for 24 hours incubation period with 6 hours intervals along with Raman signal enhancement factor variation within the said period (C)Variation in different intracellular components peaks of immobilized fibroblast cells based on SERS Raman spectra obtained from 24 hours samples containing different $\mathrm{C}-\mathrm{N}$ percentage N-CNP sensor platforms along with fluorescence micrograph and (D) Variation in different intracellular components peaks of immobilized HeLa cells based on SERS Raman spectra obtained from 24 hours samples containing different C-N percentage N-CNP sensor platforms along fluorescence micrograph with (E) Raman enhancement for DNA peak and protein peak.

Figure 5-7: Diagnosis of HeLa cells through relative intensity change of protein, DNA and RNA peaks of both HeLa and fibroblast cells from live Raman spectra analysis for 24 hours incubation period with 6 hours intervals. 


\section{List of Abbreviations}

3D - Three-Dimensional

CV - crystal violet

CNTs - Carbon Nanotubes

CVD - Chemical Vapor Deposition

DMEM - Dulbecco's Modified Eagle Medium

$\mathbf{E F}$ - enhancement factor

fs - Femtosecond

MHz -. Megahertz Pulse

MWCNTs - Multi Walled Carbon Nanotube

nm - Nanometer

NIH 3 T3 - Mouse Embryonic Fibroblasts

PLV - Pulsed Laser Vaporization

R6G - Rhodamine 6G

SWCNTs - Single-Walled Carbon Nanotubes

SEM - Scanning Electron Microscope

SERS - Surface Enhanced Raman Scattering

TEM - Transmission Electron Microscopy

XRD - X-Ray Diffraction

XPS - X-ray Photoelectron Spectroscopy

$\boldsymbol{\mu m}$ - Micrometer 


\section{Chapter 1}

\section{Introduction}

\subsection{Carbon nanostructures for biomedical applications}

There are astounding variety of natural and artificial carbon allotropes and at the nanoscale many of these carbon structural formations have noteworthy mechanical and physicochemical properties. These promising nano-based properties have excited the scientists to exploit these wonderful structures in prospective biomedical research. Carbon-based nanomaterials, such as carbon nanotubes $(\mathrm{CNTs})^{[1-1]}$, carbon nanowalls $(\mathrm{CNWs})^{[1-2]}$, graphene and its derivatives ${ }^{[1-3,1-4]}$, have been actively explored for different biomedical applications in the area of therapy and diagnosis. They have received considerable attention by researchers for various biomedical applications such as scaffolds in tissue engineering ${ }^{[1-5]}$, substrates for stem cell differentiation ${ }^{[1-4]}$, components of implant devices ${ }^{[1-6]}$, diagnostic tools and chips ${ }^{[1-7]}$, biological imaging ${ }^{[1-8]}$, drug delivery carriers ${ }^{[1-}$ 9], and antibacterial materials ${ }^{[1-10]}$. Carbon based nanomaterials due to their unique physiochemical properties, such as surface area, superior electrical and thermal conductivity and biological behavior, including high chemical inertness or mechanical stability ${ }^{[1-11,1-12]}$ showed increased exploration into the biomedical filed.

\subsection{Carbon nanostructures in tissue engineering and therapeutic applications}

A scaffold for tissue engineering requires attraction, adhesion and proliferation of cells. Recently carbon nanostructures/nanomaterials gain attention in tissue engineering. However, cytotoxicity or/and chemical inertness of these nanomaterials is a barrier to positive cell response. Conjugation with bio-molecules/chemicals/proteins etc. provide required biocompatibility and attained 
favorable cues towards attraction, adhesion and proliferation of cells. Figure 1-1 shows the use of carbon nanostructure e.g. carbon nanotubes (CNTs) for control cell growth ${ }^{[1-13]}$. Graphene showed positive response in the focal adhesion and proliferation of fibroblast and osteoblast cells ${ }^{[1-14,1-15]}$.

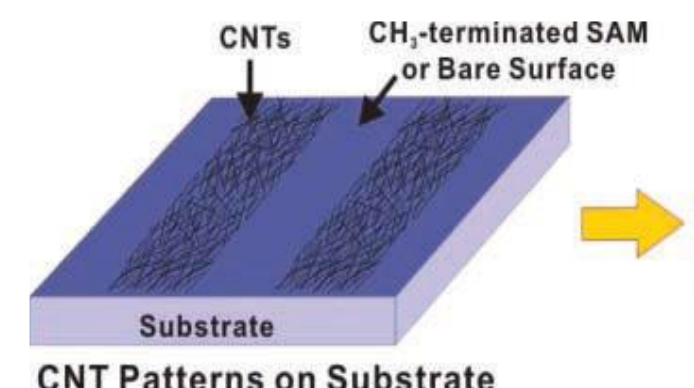

CNT Patterns on Substrate

( $\mathrm{Au}$, glass, or $\mathrm{SiO}_{2}$ )

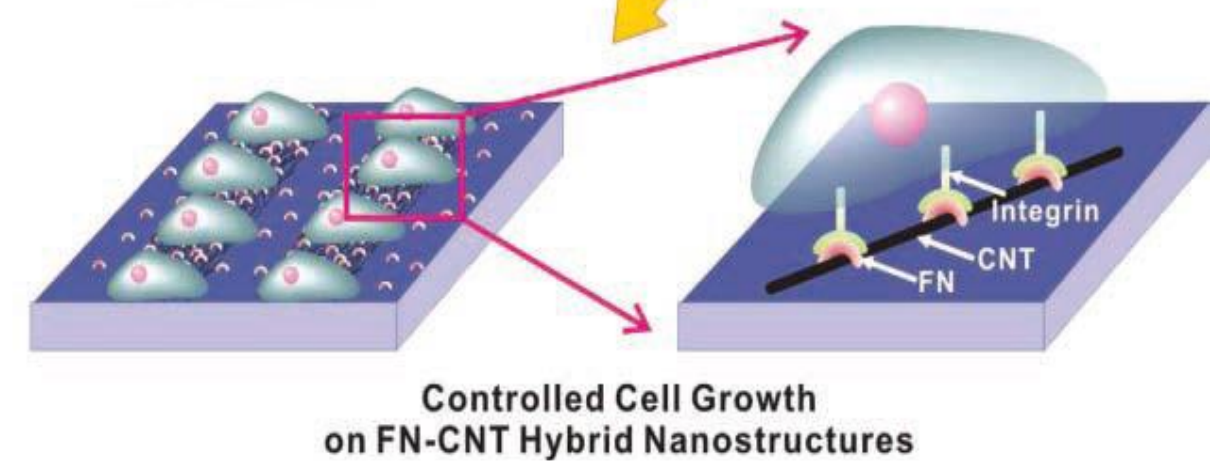

Figure 1-1: Schematic diagram depicting the controlled cell growth on fibronectin-carbon nanotube (FN-CNT) hybrid nanostructures (courtesy [1-13])

Researchers also actively explored carbon nanostructures as supportive substrates as well as excipients for multi-functional drug delivery systems. Their physicochemical properties offer both covalent and non-covalent functionalization with different functional groups. At the same time, they can carry several moieties and can also passively cross the membranes of many different types of cells ${ }^{[1-16,1-17]}$. All these properties make carbon nanostructures a good contender for therapeutic applications. Different research groups have explored CNTs for diverse biomedical application especially in cancer treatment ${ }^{[1-1]}$, where CNTs act as nanocarriers functionalized with a variety of biomolecules and drugs. There has been very limited reporting on the direct interaction of CNTs with cancer cells because CNTs contain amorphous carbon impurities or the remains of catalytic 
particles of prepared materials that induce cytotoxicity, which can influence cell culture efficacy ${ }^{[1-18]}$. Figure 1-2 shows apoptosis of HeLa cell under RF excitation created by graphitic carboncoated ferromagnetic cobalt nanoparticles $(\mathrm{C}-\mathrm{Co}-\mathrm{NPs})^{[1-19]}$.

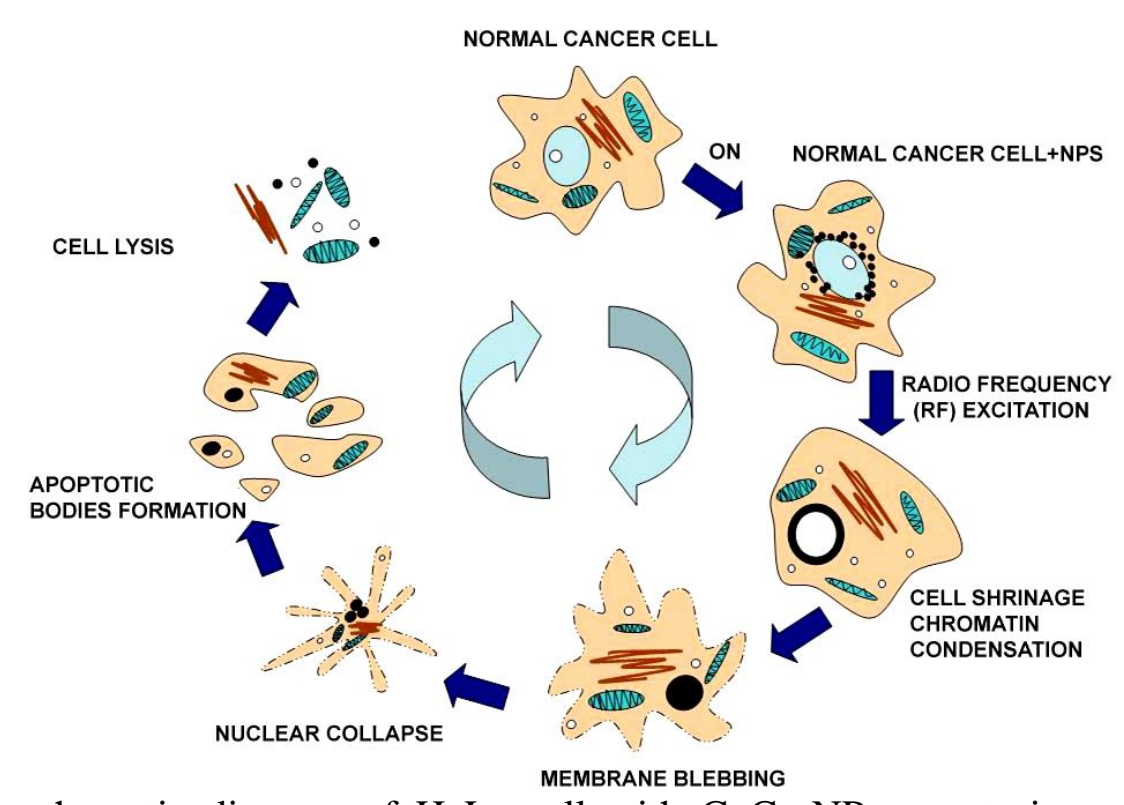

Figure 1-2 A schematic diagram of HeLa cell with C-Co-NPs apoptosis process under RF excitation. (courtesy [1-19])

\subsection{Carbon nanostructures in bio-sensing and diagnostics applications}

Carbonaceous nanostructures due to their different anisotropies and surface properties are good candidate to act as a bio-sensor. With the continuous progress of nanotechnology in material science, carbon nanomaterials, especially carbon nanotubes $(\mathrm{CNTs})^{[1-20]}$, graphene ${ }^{[1-21]}$ and graphene oxides ${ }^{[1-22]}$ have been actively explored by the researchers in bio-sensing applications. Graphene and its derivatives (specially graphene oxide) conjugated with numerous materials such as conducting polymers, proteins, noble metals and act as bio-sensing platform for detection of platelet derived microparticles ${ }^{[1-22]}$, Escherichia coli (E. Coli) ${ }^{[1-23,1-24]}$ and the human papillomavirus DNA ${ }^{[1-25]}$. Figure 1-3 shows schematic design of graphene based biosensors. 


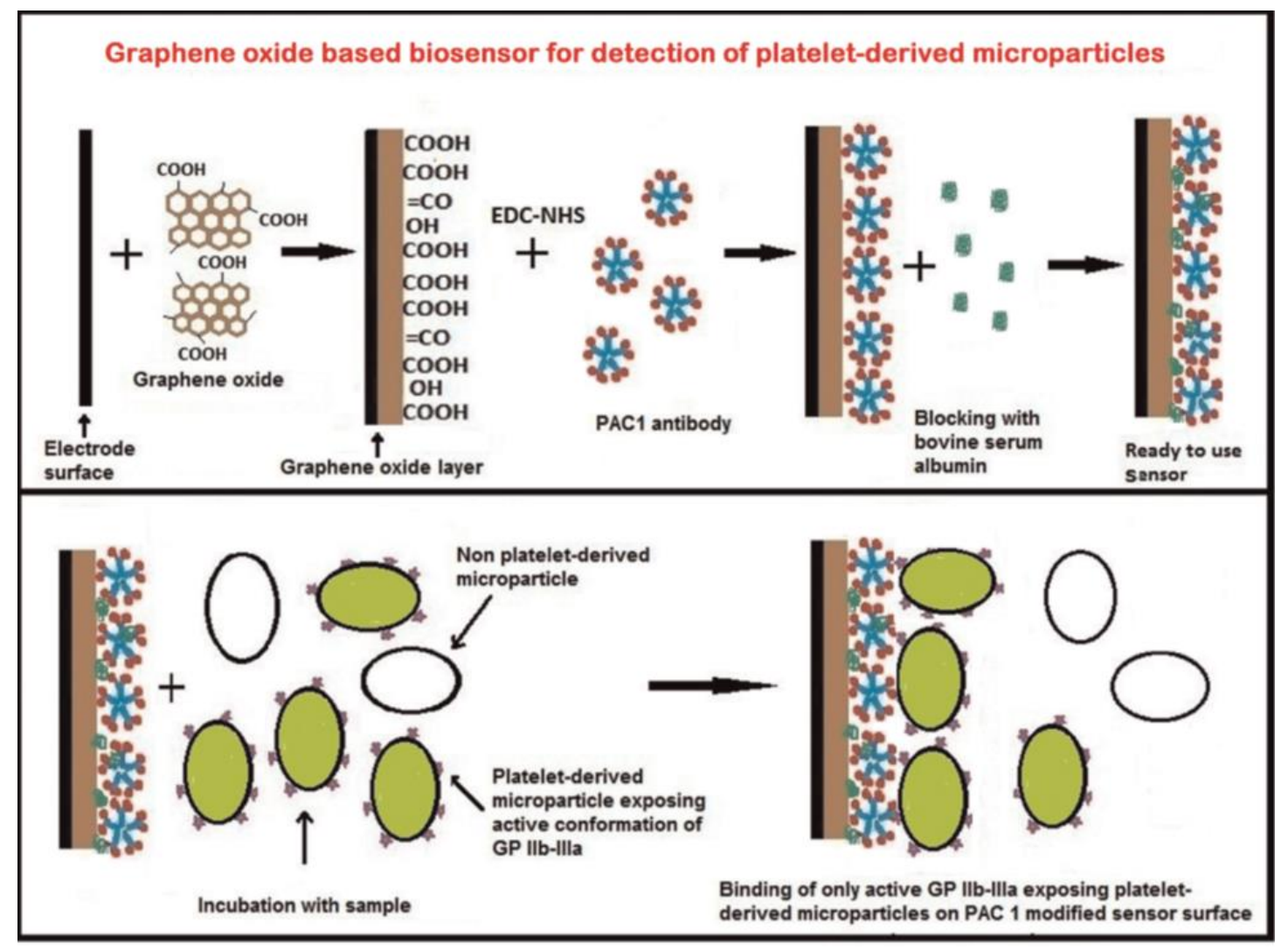

Figure 1-3 Schematic design of graphene oxide based biosensor. (courtesy [1-22])

Recently, different researchers also used carbon based nanostructures/nanomaterials for cancer diagnostics. With noble metal (Au, Ag) nanoparticle conjugation, CNTs achieved detection, differentiation and therapy of cancer cells ${ }^{[1-26]}$. Graphene was conjugated with gold nanoparticles and differentiation of human breast normal, cancer and cancer stem cells was achieved ${ }^{[1-27]}$.

\subsection{Prevailing synthesis trends for carbon nanostructures/nanomaterials}

Different multi step approaches with vacuum or controlled environment were so far employed to fabricate carbon based nanomaterials/nanostructures. The arc discharge method and different types of chemical vapour deposition (CVD) techniques are most commonly utilized techniques for large scale production of carbon nanotubes and other related type of carbon nanomaterials. Among these two methods, the arc discharge method is the oldest one used for carbon nanotube production. The same method was used to produce fullerenes and later improved for synthesis of multiwall 
(MWNT) and single wall (SWNT) carbon nanotubes. Carbon nanotubes (CNTs) fabrication process requires transition metal catalysts such as $\mathrm{Fe}$ or $\mathrm{Co}$ and Mo which subsequently need to be separated from the produced raw CNTs and complex purification process is essential to get pure CNTs appropriate for biological applications. Exfoliation is usually employed to modify the surface of graphite to produce graphene ${ }^{[1-28]}$.

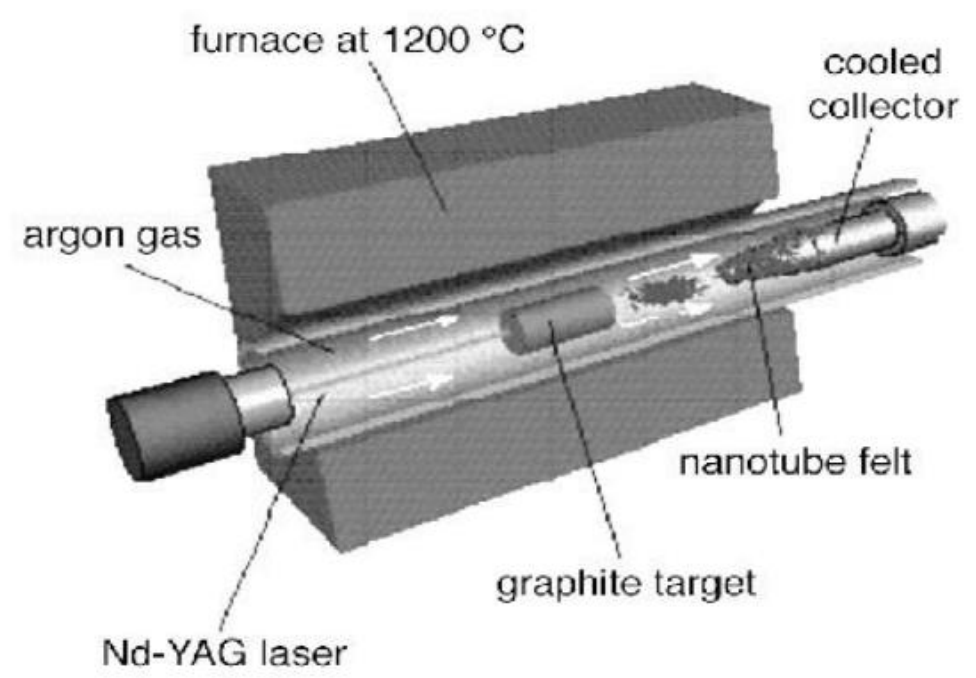

Figure1-4 A schematic synthesis apparatus for classical laser ablation used for production of carbon nanotube ${ }^{[1-29]}$. (courtesy [1-29])

The pulsed laser vaporization (PLV), or laser ablation, is a well-known method currently being used to produce Carbon nanomaterials. Creation of different type of carbon nanomaterials (like one layer/multi-layer nanotubes, nanohorns, nanofibers, nanoclusters etc. With different size) depends on the experimental conditions (like vacuum or buffer gas, presence of catalyst) and laser sources. Different researchers have employed different type of laser like Nd: YAG Laser, KrF laser, $\mathrm{CO}_{2}$ Laser etc. for ablation ${ }^{[1-30,1-31,1-32]}$. These methods vaporize the carbon and later deposit it onto a substrate.

The ultrashort pulsed laser synthesis has an important advantage over the other pulsed laser is that there is little or no collateral damage in dielectric materials. The pulse duration in the Ultrashort 
laser interaction with a solid target appears to be shorter than all distinctive relaxation times: the electron heat conduction time, the electron to ion energy transfer time and consequently the hydrodynamic or, the expansion time.

\subsection{Summary}

Carbonaceous nanomaterials have showed numerous inspiring developments concerning cancer research especially in diagnosis and therapy. In most cases, however, these nanomaterials must be functionalized biologically or chemically due to their biological inertness or possible cytotoxicity. So far research emphasis was concentrated on treating C-C molecular structure based carbon nanomaterials/nanostructures as a carrier/skeleton and with the aid of further surface modification through functionalization with chemical agents or biomolecules tried to attain different therapeutic/diagnostic applications. There is a research potential to create an inherently functionalized bio-active carbon nanostructure that can stimulate affirmative cell response and have prospective biomedical applications in terms of therapy and diagnosis.

\subsection{Research objectives}

Ultrafast pulsed laser ionization assisted synthesis mechanism selectively modify the nonresponsive $\mathrm{C}-\mathrm{C}$ phase of graphite and offers promise in the generation of new bioactive carbonaceous nanostructures/nanomaterials with unique shape, size and physicochemical properties. The main objectives of this research are given below:

- To synthesize 3D self assembled nanocarbon network using ultrafast pulsed laser ionization assisted synthesis

- To explore the therapeutic functionality of the created nanocarbon using HeLa and Fibroblast cells. 
- To identify the diagnostic capability of the created nanocarbon through SERS based label free non-plasmonic in vitro sensing and early detection of HeLa and fibroblast cells.

\subsection{Organization of the thesis}

Manuscript based style has been followed to organize the thesis.

Chapter 2 describes the self-biofunctionalization of non-responsive carbon phase and development of a new biocompatible 3D carbon nano-network with a bioactive phase that significantly promotes fibroblast cell adhesion. This cytophilic bio-nanocarbon does not require any subsequent biological/chemical treatments and considerably stimulates fibroblast cell adhesion and subsequent cell proliferation.

Chapter 3 reveals the dual functionality i.e. cytophilic to fibroblasts but cytotoxic to HeLa cells of carbon nanoribbon network platform having a combination of $\mathrm{C}-\mathrm{C}$ and $\mathrm{C}-\mathrm{O}$ bond architecture. The said platform acts as a favorable attractor and a proliferation-promoting substrate for both fibroblasts and HeLa cells. With the passage of time it induces cell-specific anticancer cytotoxicity on HeLa cells and lead to cell death (apoptosis) but no harm on mammalian fibroblast cells and develops tissue like structures.

Chapter 4 Introduces a label free unique non-plasmonic SERS based bio-sensing platform using SERS active 3D interconnected nanocarbon web for in-vitro detection and differentiation of HeLa and fibroblast cells. The sub-10 nanometer structure of the said nanocarbon has self-internalization capacity which results in live cell Raman sensing. Individual detection of HeLa and fibroblast cells from chemical fingerprints of intracellular components like DNA/RNA, protein and lipids of both cell lines have been revealed through Raman spectroscopic peak differences. Additionally, there is a guideline for identification of each cell health from spectral responses. 
Chapter 5 delineates a nitrogen incorporation-assisted approach to create a new nitrogen enriched 3D carbon nanoprobe sensor platform and demonstrate a label free non-plasmonic SERS based unique technique of early detection of HeLa cancer cells by intracellular sensing. This platform demonstrates favorable microenvironment for cell adhesion and SERS hot spots is created inside the HeLa and fibroblast cells through internalization of nitrogen enriched 3D carbon nanoprobe unit and thereby achieves SERS based live cells Raman sensing. The revealed fingerprints of the intracellular components (protein, DNA, RNA etc.) of HeLa and fibroblasts cells guide early detection of HeLa cells and thereby cancer diagnosis.

Chapter 6 summarizes the main results and proposes future direction for this research. 


\section{Chapter 2}

\section{Biofunctionalized 3-D Carbon Nano-Network Platform for Enhanced Fibroblast Cell Adhesion}

Published in Nature Scientific Reports, March 2017

Carbon nanomaterials have been investigated for various biomedical applications. In most cases, however, these nanomaterials must be functionalized biologically or chemically due to their biological inertness or possible cytotoxicity. Here, we report the development of a new carbon nanomaterial with a bioactive phase that significantly promotes cell adhesion. We synthesize the bioactive phase by introducing self-assembled nanotopography and altered nano-chemistry to graphite substrates using ultrafast laser. To the best of our knowledge, this is the first time that such a cytophilic bio-carbon is developed in a single step without requiring subsequent biological/chemical treatments. By controlling the nano-network concentration and chemistry, we develop platforms with different degrees of cell cytophilicity. We study quantitatively and qualitatively the cell response to nano-network platforms with NIH-3T3 fibroblasts. The findings from the in vitro study indicate that the platforms possess excellent biocompatibility and promote cell adhesion considerably. The study of the cell morphology shows a healthy attachment of cells with a well-spread shape, overextended actin filaments, and morphological symmetry, which is indicative of a high cellular interaction with the nano-network. The developed nanomaterial possesses great biocompatibility and considerably stimulates cell adhesion and subsequent cell proliferation, thus offering a promising path toward engineering various biomedical devices. 


\subsection{Introduction}

Carbon based nanomaterials have received considerable attention by researchers for various biomedical applications such as scaffolds in tissue engineering ${ }^{[2-1]}$, substrates for stem cell differentiation ${ }^{[2-2]}$, components of implant devices ${ }^{[2-3]}$, diagnostic tools and chips ${ }^{[2-4]}$, biological imaging ${ }^{[2-5]}$, drug delivery carriers ${ }^{[2-6]}$, and antibacterial materials ${ }^{[2-7]}$. To date, numerous research groups have investigated the response of mammalian cells to carbon nanomaterials in terms of cell viability, adhesion, and proliferation, with most of the recent works have been concentrated on carbon nanotubes $(\mathrm{CNTs})^{[2-8]}$ and graphene ${ }^{[2-9]}$. Though the carbon nanomaterials have shown some satisfactory results for different cell lines in terms of biocompatibility, their health and safety issues have been a concern, so they have been widely examined for their toxicity. There are still contradictory reports for example for CNTs that show strong cytotoxic activities in some cases and ability to cell growth in some other cases ${ }^{[2-10,2-11]}$. Therefore, these nanomaterials usually should be purified or/and functionalized with chemical/biological molecule for positive cell response owing to their possible cytotoxicity or/and chemical inertness.

In general, the purity of materials influences their cytotoxicity and thus cell responses. As for carbon nanomaterials, the purity particularly differs in materials obtained from carbon nanocompounds ${ }^{[2-12]}$. Great number of research works have pointed out that one of the most important factors contributing to the CNT toxicity is impurities that are introduced during synthesis and purification procedures. For instance, CNTs can be produced by several techniques, one of which for large-scale production is chemical vapor deposition (CVD). During CVD, contamination of CNTs by catalyst residues is unavoidable ${ }^{[2-13]}$. These impurities lead to cytotoxicity of CNTs. In addition, amorphous carbon is considered an impurity that might result in the toxicity of carbon nanomaterials ${ }^{[2-12,2-13,2-14]}$. The impurities thus affect cell responses to the surfaces and eventually 
influence the cell cultures efficiency. Therefore, effective functionalization processes are usually necessary to purify CNTs while sustaining their original structures ${ }^{[2-13]}$. For instance, Mottaghitalab et al. ${ }^{[2-15]}$ showed that treating single-walled carbon nanotubes (SWCNTs) substrate with the extracellular matrix component fibronectin could reduce these unfavorable characteristics, thus enhancing cellular attachment. In another study, Correa-Duarte et al ${ }^{[2-16]}$ showed that a 3-D network of interconnected multi-walled carbon nanotube (MWCNTs) functionalized in an acid solution to generate carboxylic groups was favorable for adhesion and growth of mouse fibroblast cell line L929. Nevertheless, even after the treatment processes, contaminations that increase cytotoxicity may still remain in the resulting product. Besides, the purification method itself may introduce unwanted toxic substances to the nanotubes ${ }^{[2-12]}$. In addition, the biofunctionalization stage would add another step to the multistep process of CNT synthesis.

Unlike CNTs, graphene has a much simpler structure and can also be synthesized as a relatively pure layer, which makes it more suitable substrate for the cell growth and differentiation. Indeed, previous studies involving adherent cells have reported the positive impact of graphene on the cell functions ${ }^{[2-12]}$. Ryoo et al. ${ }^{[2-17]}$ employed a glass cover slip coated with a thin film of graphene and/or MWCNTs to adhere NIH-3T3 mouse fibroblasts cells. They showed that graphene improved gene efficiency and focal adhesion and proliferation of fibroblast cells. Aryaei et al. ${ }^{[2-}$ ${ }^{18]}$ concluded that graphene did not possess any toxic effect on osteoblasts and that the cell adhesion was improved with graphene-coated substrate. In orthopedic applications, graphene polymer composites have also been designed and evaluated ${ }^{[2-19,2-20]}$. Depan et al. reported that the presence of graphene oxide in chitosan-graphene oxide scaffolds showed enhanced biological responses of osteoblasts, such as cell adhesion, proliferation, growth, and proved its influence in cell scaffold interactions ${ }^{[2-19]}$. Shan et al. synthesized poly-L-lysine-functionalized graphene a biocompatible 
and relatively friendly environment due to a large number of free active amino groups ${ }^{[2-21]}$. Yet, several researchers have reported that graphene can induce oxidative stress in cultured cells due to the formation of reactive oxygen species on its surface ${ }^{[2-22]}$. The induced oxidative stress is believed to contribute to the toxicity of graphene as nanomaterial, so despite positive cell adhesion for particular cell lines, their cytotoxic behavior originated from oxidative stress requires further functionalization ${ }^{[2-12,2-23]}$. For instance, several studies have showed that biocompatible graphene could be achieved by chemical functionalization using polymers such as poly ( $\varepsilon$-caprolactone $)^{[2-}$ ${ }^{24]}$ or poly-L-lysine ${ }^{[2-21]}$.

All in all, most carbon nanomaterials/nanostructures synthesized so far require further treatments to increase biocompatibility and to attract cell response. Hence, there is a need for a bioactive carbon with natural functionalization that can trigger cell adhesion and subsequent cell proliferation without the necessity for additional chemical or biological surface treatments.

In this work, we have introduced a novel approach to transform the non-responsive carbon phase of graphite substrate to bio-active carbon phase without the use of post-biological or -chemical treatments. We have utilized a self-assembled bottom-up approach using femtosecond laser ionization of graphite to bio-functionalize graphite substrate through creation of 3-D bio-carbon nano-network platforms. The nano-network is considered 3-D because it is composed of 10-20- $\mu$ $\mathrm{m}$ thick multilayer interwoven nanofiber materials. Laser ionization of graphite, which is performed at ambient condition without the need for vacuum settings, leads to graphite ionization and later formation of nano-network platform, which concurrently alters chemistry and nanotopography of the surfaces. The proposed laser ionization technique is performed in ambient atmosphere and requires no catalyst or background gas (and no chamber). Therefore, no impurity is introduced to the carbon nanomaterial, and thus no post-purification step will be needed. The 
laser processing parameters can be tuned to create 3-D bio-carbon nano-network platform with desired density and chemistry. The morphology and physicochemical properties of the 3-D biocarbon nano-network platform have been analyzed using scanning electron microscopy (SEM), field-emission scanning electron microscopy (FE-SEM), energy-dispersive X-ray spectroscopy (EDX), X-ray diffractometer (XRD), X-ray photoelectron spectroscopy (XPS), and micro-Raman spectroscopy. Cell-material interaction and biocompatibility of these 3-D bio-carbon nanonetwork platform have been investigated with NIH-3T3 fibroblasts (primary cells of connective tissue). Adhesion and morphology of the fibroblast cells have been studied quantitatively and qualitatively using SEM analysis and fluorescence microscopy (FM). Since cell adhesion is a key factor in the subsequent cell activity such as proliferation, protein synthesis, and tissue generation, the significant cell-adhesion-promotor property of the developed nano-network platform suggests considerable outlook for developing numerous carbon-based biomedical devices. Moreover, the simplicity and versatility of the proposed laser technique would make it a promising single-step approach to create biocompatible carbon nano-network platforms through functionalizing the nanotopography and chemistry of graphite substrates.

\subsection{Experimental Method}

\subsubsection{Carbon nano-network platform fabrication}

Isomolded, very fine grain, high strength graphite plate (Graphtek LLC, USA) with $3 \mathrm{~mm}$ thickness was cut into 4- $\mathrm{cm}^{2}$ squares. These squares were polished with sand papers (3 M Canada, 1000 grit, 2000 grit and 3000 grit) and then ultrasonically cleaned (Cole-Parmer 8890 ultrasonic cleaner) with acetone and ethanol for five $\mathrm{min} / \mathrm{step}$ and then dried at room temperature. To create a 3-D bio-carbon nano-network platform on the top of these polished surface, these substrates were irradiated with a diode-pumped, Yb-doped femtosecond laser system (Clark-MXR, Inc.; 
IMPULSE Series ultrashort pulse laser) at laser pulse repetitions of 4,8 and $26 \mathrm{MHz}$ and atmospheric condition. A fixed stage was used to mount the sample and a computer controlled high precision 2-D translation scanner guided the incident laser beam. An array of lines with a variation of spaces in between was machined to create the 3-D nano-network. The pulse energy transferred to the substrates per unit area -laser fluence (pulse energy/effective focal spot area)varied at $4.43,2.22$, and $0.68 \mathrm{~J} / \mathrm{cm}^{2}$, referred to as high, medium, and low fluences throughout this study, in order to alter the chemistry and nanotopography the developed nano-network. The laser wavelength, pulse width, power of the incident laser beam, the laser beam scanning speed, and irradiation focal spot area were maintained constant at $1030 \mathrm{~nm}, 214 \mathrm{fs}, 15 \mathrm{~W}, 1 \mathrm{~mm} / \mathrm{s}$, and 84.62 $\times 10^{-8} \mathrm{~cm}^{2}$, respectively, for all laser pulse. The laser source parameters such as wavelength, pulse width, pulse repetition, and power were controlled and monitored real-time through a computer. The power output was measured before each laser machining to ensure accuracy. The machining parameters, such as laser beam speed or beam dwell time and machining path, were also controlled and monitored through a separate computer, which was connected to the scanner. The functionality and accuracy of the scanner and laser machining parameters were tested before each experiment. All parameters were recorded for each experiment to ensure repeatability and reproducibility.

\subsubsection{Morphological and physicochemical characterization of 3-D network}

The morphology of the 3-D bio-carbon nano-network platform was examined by SEM (Hitachi SU-1500) and FE-SEM (Hitachi, SU-8200). Following SEM, EDX was carried out to determine the elemental composition of irradiated graphite. Micro-Raman spectroscopy (Bruker Senterra) (the laser excitation wavelength is at $532 \mathrm{~nm}$ ), XRD (Rigaku Miniflex 600), and XPS (Thermo Fisher Scientific K-Alpha) were employed to analyze the surface chemistry and material composition of the 3-D bio-carbon nano-network platform. 


\subsubsection{Cell Culture}

NIH-3T3 mouse fibroblasts cells were grown in a tissue culture flask with Dulbecco's Modified Eagle Medium: Nutrient Mixture F-12 (DMEM/F12), 10\% fetal bovine serum (FBS) and 1\% penicillin/streptomycin maintained at $37{ }^{\circ} \mathrm{C}$ in $5 \% \mathrm{CO}_{2}$. Passages between three and six were used for cell studies.

\subsubsection{NIH-3T3 mouse fibroblast cells-material interaction}

Prior to seed the cells, the ablated samples were kept under UV light for 20 minutes. The samples then placed inside the Petri dishes containing $3 \mathrm{ml}$ of DMEM/ F12 medium and 10\% FBS per dish and NIH-3T3 mouse fibroblasts cells were seeded at a density of $10^{5}$ cells $/ \mathrm{ml}$. The Petri dishes were incubated for 24 and 48 hours. The spent medium was removed, and the samples were fixed with glutaraldehyde after incubation. Subsequently, the sample was washed twice with $1 \%$ sodium cacodylate buffer $\left(\mathrm{P}^{\mathrm{H}}\right.$ of 7.3$)$ at $4{ }^{\circ} \mathrm{C}$. Then, the cells were dehydrated through a graded ethanol series (from $10 \%$ to $100 \%$ ) for $15 \mathrm{~min}$. Then, the samples were critical point dried and before the SEM examination, samples were sputtered with a gold layer.

\subsubsection{Fluorescent staining of cells}

In the first step, paraformaldehyde was used to fix the samples. Then it was followed by incubation in skimmed milk powder to prevent non-specific binding. The samples are then incubated with Alexa Fluor 488 phalloidin (Life Technologies) to stain the actin and cytoskeleton followed by DAPI (4', 6'-diamidino-2-phenylindole, Life Technologies) to stain the nucleus. An epifluorescent Nikon E-400 microscope with FITC and DAPI filter was used, and the data were recorded using a DS-5M-U1 color digital camera (Nikon, Canada). 


\subsubsection{Statistics}

All experiments were done in triplicate, and the data represented the mean \pm standard deviation unless otherwise mentioned. The cell counting was made using SEM micrographs and image processing software.

\subsection{Results}

\subsubsection{Synthesis of 3-D nanofibrous network on Graphite substrate}

The 3-D bio-carbon nano-network platform was synthesized by ultrashort femtosecond laser processing of graphite plates at ambient condition. The schematic of the bottom-up fabrication process is represented in Fig.2-1. In general, the formation of nano-network is a function of ionization energy, which directly correlates to laser parameters, such as laser power, ultrashort pulse laser fluence, laser repetition, and scanning speed, background gas (air), and the material properties. In this study the ultrashort pulse laser fluence, which is defined as laser pulse energy per beam area, were tweaked to achieve different nanotopography and chemistry. The change in substrate chemistry is mainly due to the ionization of the graphite by high-energy femtosecond laser pulse. The concentration of created carbon nano-network $\left(\rho_{n s}\right)$ is proportionate to the number of ionized carbon nanoparticles $\left(N_{n p}\right)$ per pulse, which is directly proportional to the ultrashort pulse laser fluence, $f\left[J / \mathrm{cm}^{2}\right]=E_{p}[J] / A_{f}\left[\mathrm{~cm}^{2}\right]$, where $E_{p}$ is laser pulse energy and $A_{f}$ is effective laser beam area, and is inversely proportional to laser machining speed ${ }^{[2-25]}, \rho_{n s} \propto$ $\frac{1}{S}(f)^{1 / 2}$ The number of pulses arriving in the same area and thus the transported energy to the substrate can also be controlled with the combination of beam scanning speed and pulse repetition rate. In this study, the ultrashort pulse laser fluence of $4.43,2.22$, and $0.68 \mathrm{~J} / \mathrm{cm}^{2}$, high, medium, and low fluence, 


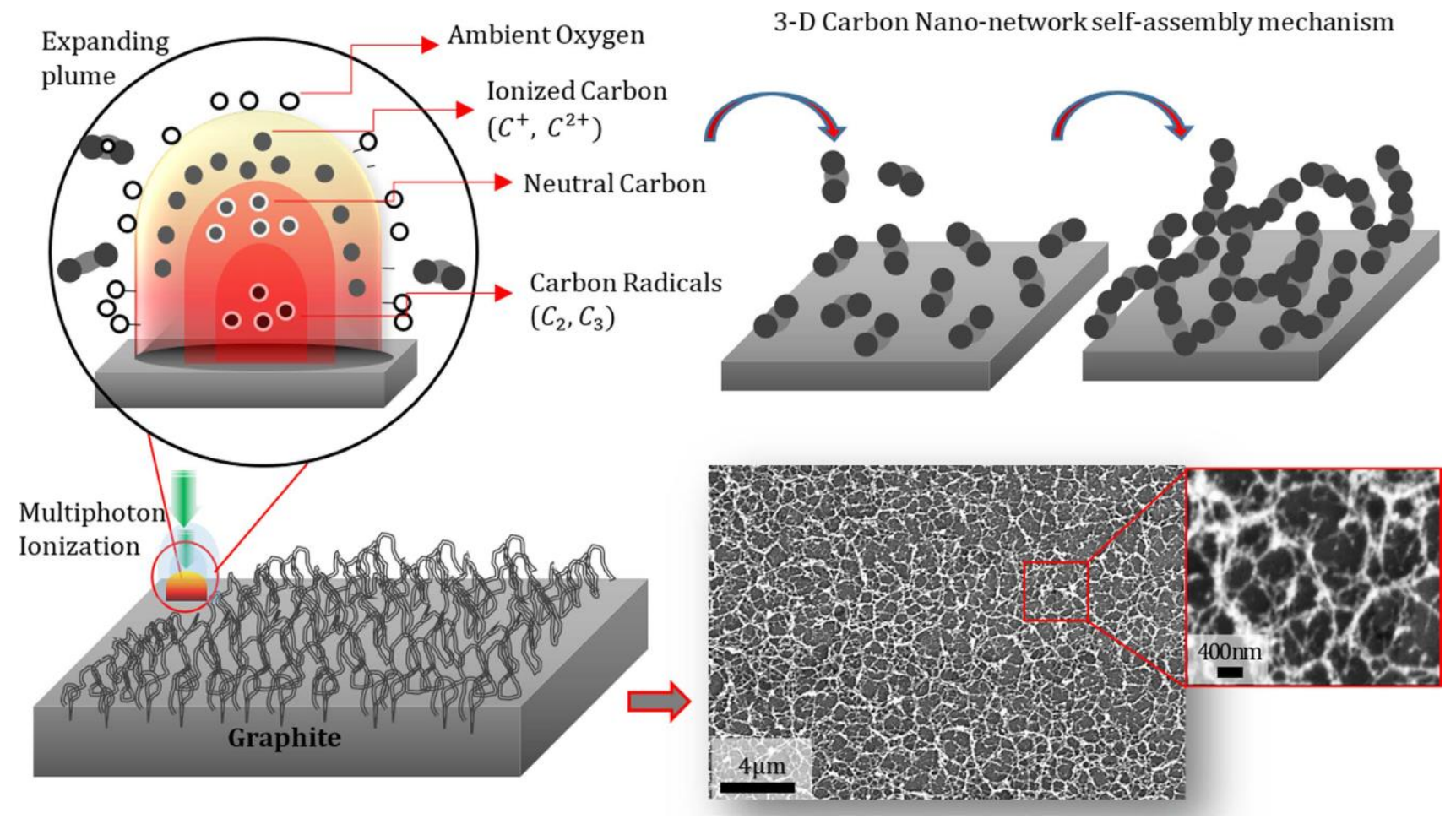

Figure 2-1: Schematic of the laser ionization of graphite substrate and the synthesis of nanonetwork platform and its corresponding SEM micrographs.

were employed to create carbon nano-network with three different density and altered chemistry.

Figure 2-2 compares SEM micrographs of a native graphite substrate and the morphology of the nano-network platforms created at low, medium and high fluence with their corresponding FESEM micrographs of their constituent nanoparticles. The SEM micrographs in Fig. 2-2 (A-D) illustrate the evolution of web-like interlinked nano-network on the native graphite surface from low to high ultrashort pulse laser fluence irradiation. In respect to surface morphology, processing with low ultrashort pulse laser fluence resulted in a less dense nanofibrous network with some degree of porosity within it (Fig. 2-2 B). With the increase of ultrashort pulse laser fluence, denser packed nanofibrous structures were synthesized as high energy per pulse led to higher surface ionization, greater nanoparticle generation in the plume, and thus denser aggregation thereof. The FE-SEM micrographs of the fabricated nano-network shown in Fig.2-2 also confirmed the 
interconnected nature of nanoparticles. Careful adjustment of laser processing parameters enabled us to achieve, in addition to nanotopography changes, desired chemistry alteration of the nanostructures.
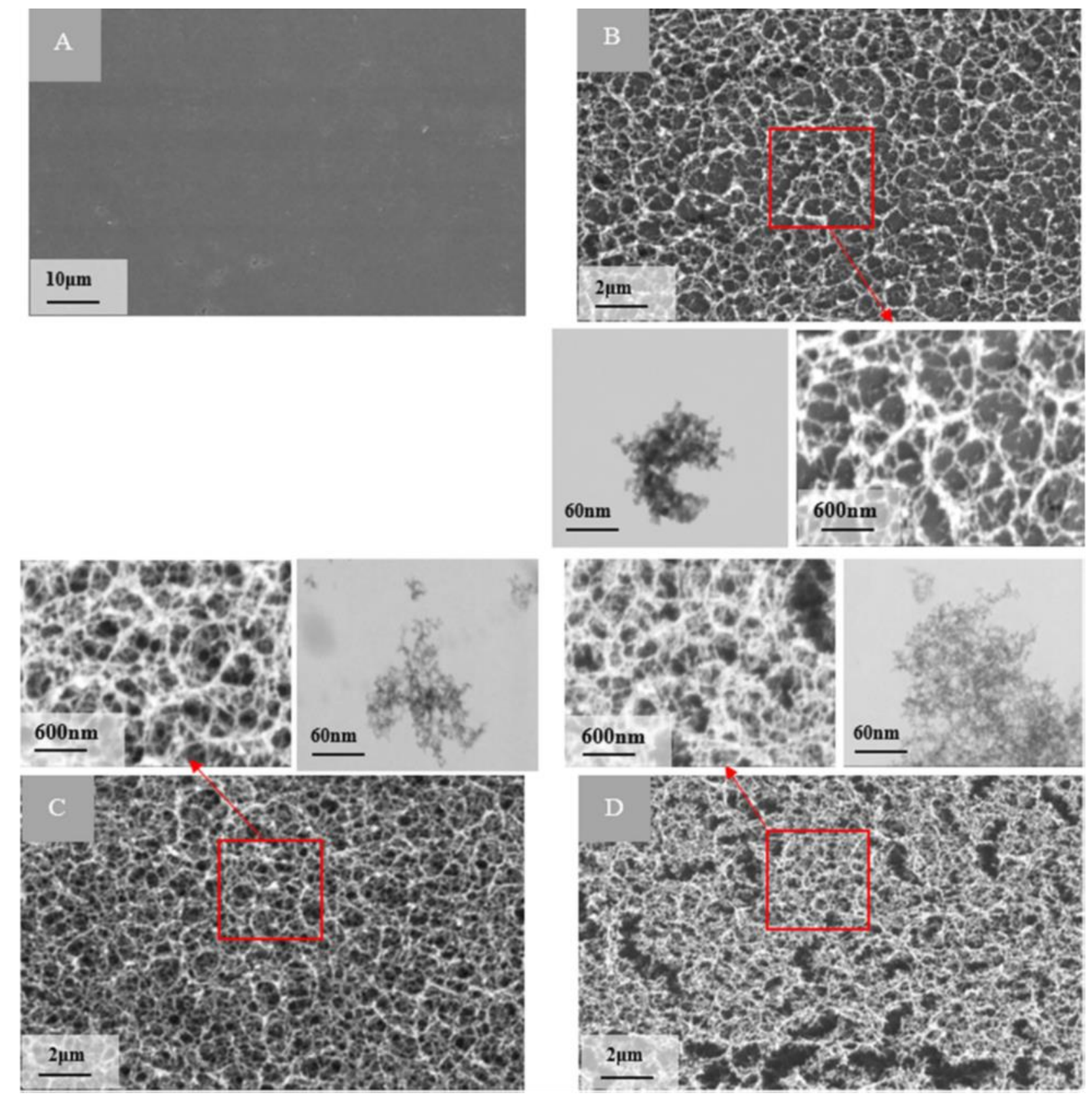

Figure 2-2: The SEM and corresponding FE-SEM micrographs of (A) native graphite substrate and the nanonetwork platforms created at (B) low (C) medium, and (D) high laser fluence. 
Micro-Raman, XPS, XRD, and EDX analyses were conducted to examine the material chemistry of the nano-networks. Micro-Raman spectra of graphite are characterized by two zone center optic phonons that propagate along the crystalline $\mathrm{c}$ axis ${ }^{[2-26,2-27]}$. The configuration of the $\mathrm{sp}^{2}$ sites in the $\mathrm{sp}^{2}$ bonded clusters evaluates the dependency of the peaks in the spectrum. $\mathrm{E}_{2 \mathrm{~g}}$, the doubly degenerate in-plane optical vibration, is Raman active. For graphite, there are two different $E_{2 g}$ modes. The difference in energy between the two $\mathrm{E}_{2 \mathrm{~g}}$ modes has assigned the Raman Bands that is observed in the single crystal of graphite ${ }^{[2-28,2-29]}$. $\mathrm{G}$ band arises due to the high energy phonon $\left(E_{2 g}\right)$ which corresponds to in-plane, covalent carbon- carbon bond stretching. The low-energy phonon $\left(\mathrm{E}_{2 \mathrm{~g}}\right)$ corresponds to a shearing motion of the weak, inter-planar van der Waals bonds, which shows D band. Figure 2-3A compares the Raman spectra of a native graphite substrate and the nanofibrous network created with different ultra-short pulse laser fluence. For both native surface and nano network surface, D band peak and G band peak appeared in the range of 1330 $\mathrm{cm}^{-1}$ to $1365 \mathrm{~cm}^{-1}$ and $1555 \mathrm{~cm}^{-1}$ to $1565 \mathrm{~cm}^{-1}$, respectively ${ }^{[2-30]}$. Two other prominent peaks also appeared at $2314 \mathrm{~cm}^{-1}$ and about $2700 \mathrm{~cm}^{-1}$, which are known as $\mathrm{D}^{\prime}$ and $\mathrm{G}^{\prime}$, and have been previously reported by other researchers ${ }^{[2-28]}$. For characterizing nano graphitic structures, the determination of the in-plane crystallite size, which is referred to as $L a$, is widely used. An empirical relationship relating $L a$ and $\mathrm{Id} / \mathrm{Ig}$ have been used where $\mathrm{I}_{\mathrm{D}}$ and $\mathrm{I}_{\mathrm{G}}$ are the intensities of D and G bands. Earlier, Tuinstra and Koenig ${ }^{[2-29]}$ performed Raman and XRD studies on different graphitic samples with different in-plane crystallite sizes $L a$ and concluded that the ratio of the intensities of $\mathrm{D}$ and $\mathrm{G}$ bands, $\mathrm{Id} / \mathrm{Ig}$, was inversely proportional to the crystallite sizes $L a$. Based on the experiment of Tuinstra and Koenig ${ }^{[2-29]}$, later L. G. Cancado et al. ${ }^{[2-31]}$ developed a general equation of the in-plane crystallite size $L a$ of crystalline graphite as follows: 


$$
\mathrm{L}_{\mathrm{a}}(\mathrm{nm})=\left(2.4 \times 10^{-10}\right) \lambda_{l}^{4}\left(\frac{\mathrm{I}_{D}}{I_{G}}\right)^{-1}
$$

Where, $\lambda_{1}$ is the laser excitation wavelength, $\mathrm{I}_{\mathrm{D}}$ and $\mathrm{I}_{\mathrm{G}}$ are the intensities of $\mathrm{D}$ and $\mathrm{G}$ band. Using $I_{d}$ and $I_{g}$ intensities of $D$ and $G$ band and Equation 1, the in-plane crystallite sizes $L a$ were calculated, as detailed in Table 2-1. The dependence of the intensities ratio on the crystallite size and effect of the ultrashort pulse laser fluence on the nano-network crystallite formation was graphically presented in Fig.2-3B and C.

A

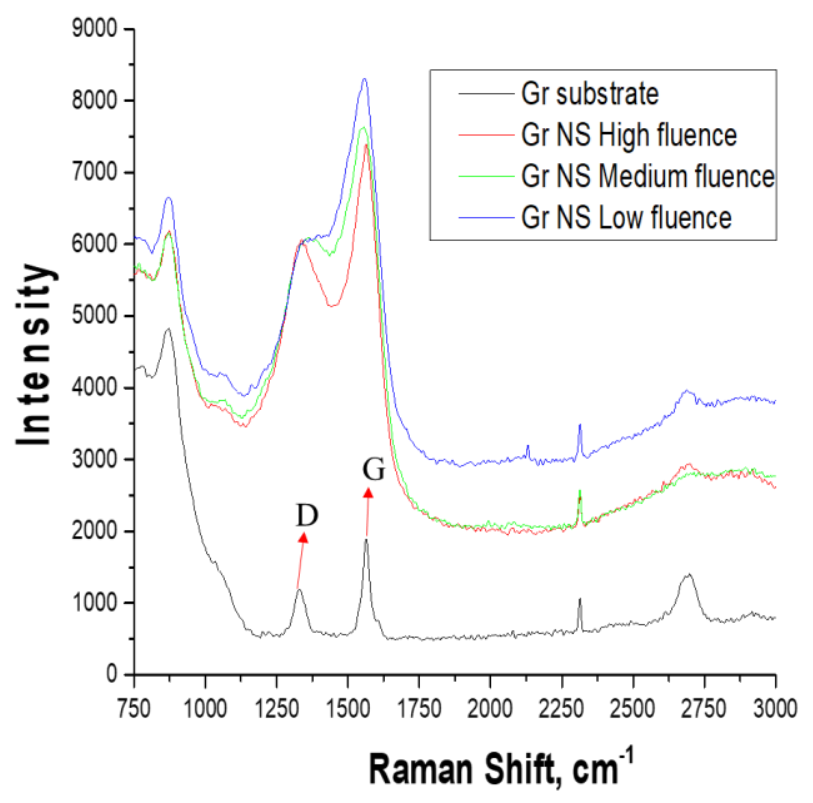

B

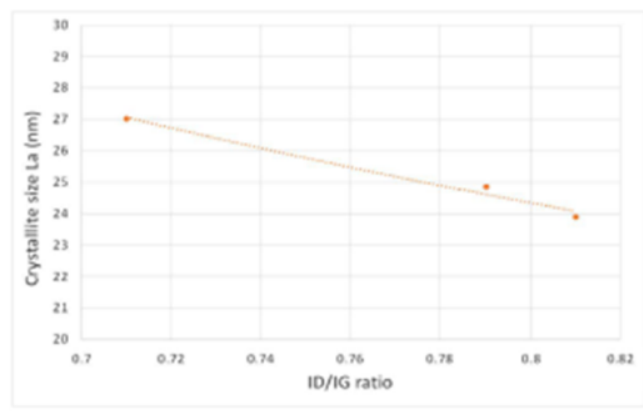

C

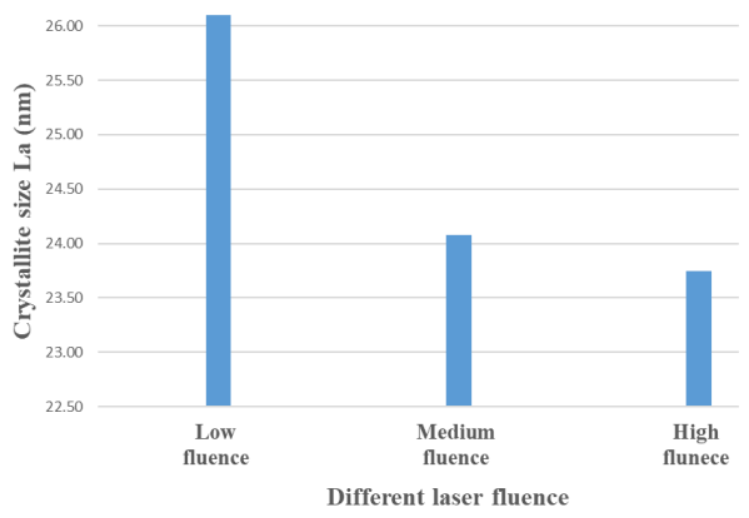

Figure 2-3: (A) A comparison of Micro-Raman spectra of native graphite and the nano-network platform synthesized at different laser fluences, (B) the relationships between crystallite size La and $\mathrm{I}_{\mathrm{D}} / \mathrm{I}_{\mathrm{G}}$ ratio, and $(\mathrm{C})$ the influence of the laser fluences on the crystallite size La. The $\mathrm{I}_{\mathrm{D}} / \mathrm{I}_{\mathrm{G}}$ ratio is recorded from the Micro-Raman spectra, and it is used to calculate crystallite size La using Equation 1. 
Table 2-1. Crystallite size (La) calculation using Equation 1 and the Id and Ig intensities from Micro-Raman spectra of nano-network platforms developed at different fluences.

\begin{tabular}{|l|c|c|c|c|c|}
\hline & $\mathbf{I}_{\mathrm{d}}$ & $\mathrm{I}_{\mathrm{B}}$ & $\mathrm{I}_{\mathrm{d}_{\mathrm{B}}}$ & $\mathbf{\lambda}$ & $\mathbf{L}_{\mathbf{a}}$ \\
\hline Low & 6339.16 & 8913.18 & 0.71 & 532 & 27.01 \\
\hline Medium & 6549.79 & 8342.73 & 0.79 & 532 & 24.85 \\
\hline High & 5975.28 & 7412.35 & 0.81 & 532 & 23.89 \\
\hline
\end{tabular}

XPS, FE-SEM-EDX, and XRD analyses were conducted to characterize the surface chemistry and crystal structure of the synthesized 3-D bio-carbon nano-network platform. Figure 2-4 presents the characteristic XPS spectra of carbon nano-network platform synthesized at high fluence and native graphite substrate, which compare the chemical state of carbon atoms in these two different positions. Two characteristic peaks were observed at $285.08 \mathrm{eV}$ and $533.08 \mathrm{eV}$ for both the carbon nano-network platform and the native graphite substrate. The peak positions $285.08 \mathrm{eV}$ and 533.08 $\mathrm{eV}$ in both the carbon nano-network platform and the native graphite substrate can be ascribed to $\mathrm{C} 1 \mathrm{~s}$ and $\mathrm{O} 1 \mathrm{~s}$, respectively ${ }^{[2-32,2-33]}$. The quantitative analysis of the major elements as depicted from the graph (Fig.2-4 C) indicates the increased percentage of oxygen in the synthesized carbon nano-network platform in comparison with native substrate, which is the result of surface ionization in ambient atmosphere. There was a slight, insignificant variance in oxygen content in the created nano-network platforms with the medium and low fluence.

As shown in the Fig.2-5A, the FE-SEM-EDX analysis of the 3-D bio-carbon nano-network platform patterns confirmed they mainly consisted of carbon with a small trace of oxygen. Figure 2-5B compares the XRD patterns of the native graphite substrate and the 3-D bio-carbon nanonetwork platform developed on graphite substrates. XRD patterns revealed the presence of standard nano-graphite crystalline orientations as mentioned in Fig.2-5B with the exception peak 
at $2 \theta \approx 32$, which is attributed to crystalline carbon (C-00-046-0943). However, A Marcu et al. ${ }^{[2-}$ ${ }^{34]}$ reported a similar peak with an almost identical angular position corresponding to the diamond maximal intensity peak (Miller indices - 022).

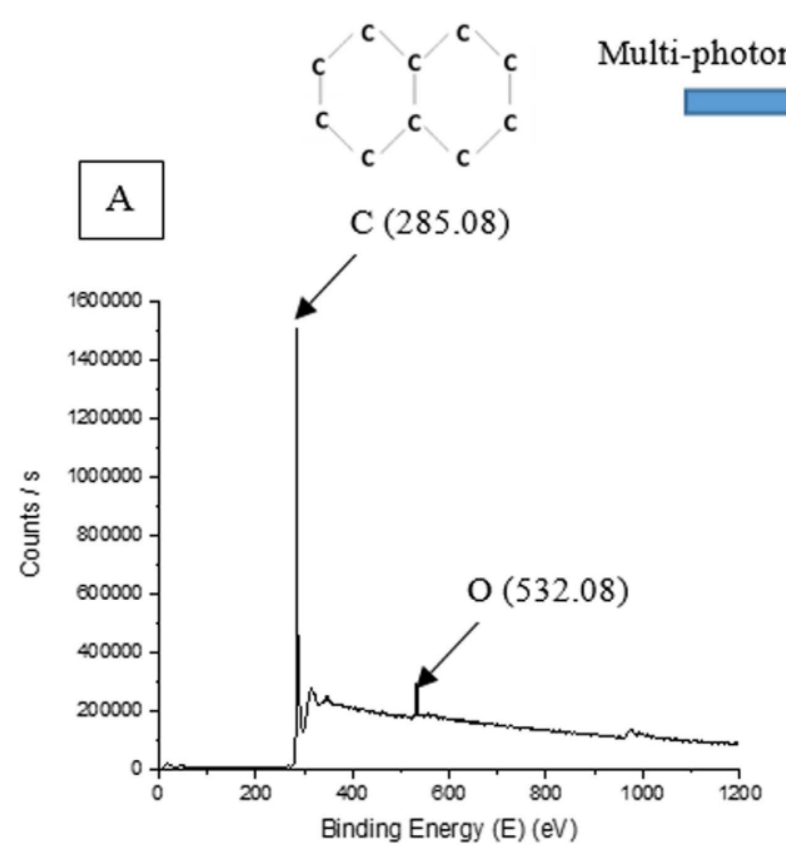

Native Graphite Substrate
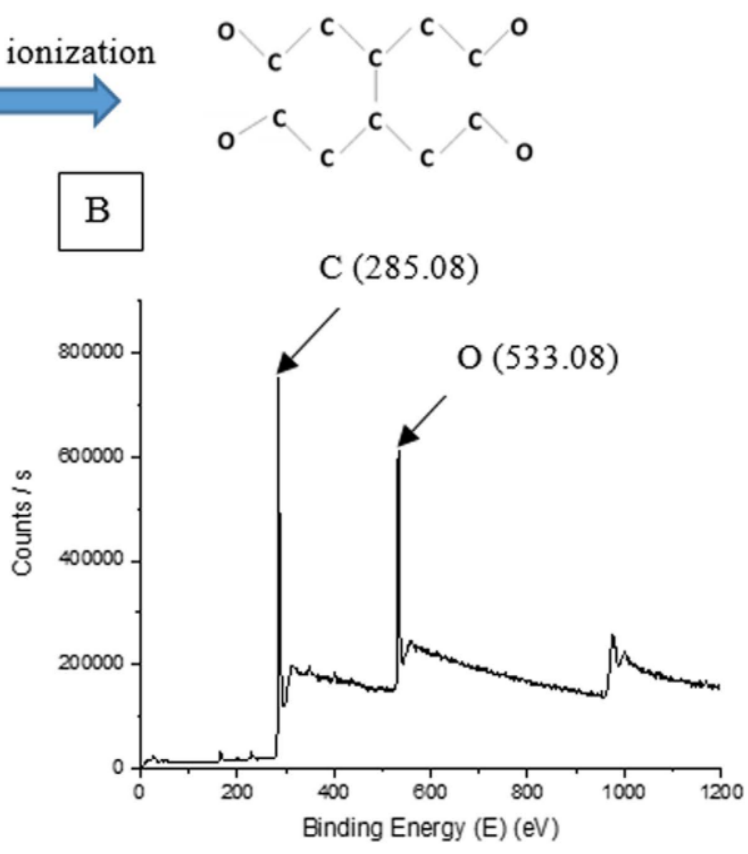

Carbon nano-network Platform

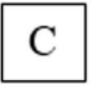

Qantitative analysis of major elements
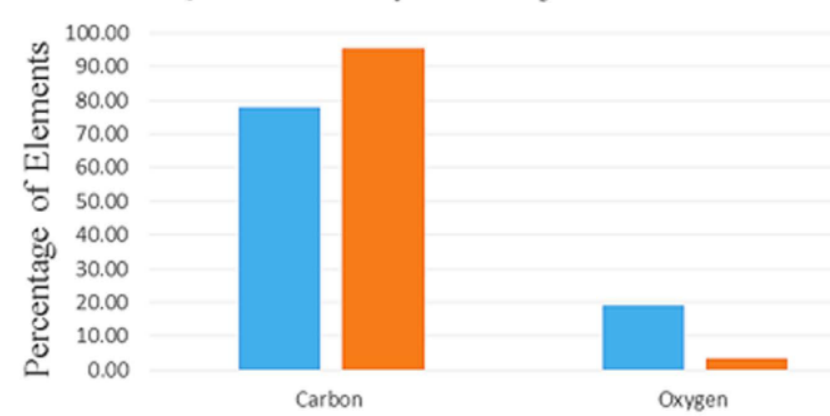

॥ Carbon nano-network Platform $=$ Native Graphite Substrate

Figure 2-4: XPS spectra of (A) native graphite substrate and (B) the nano-network platform; (C) XPS quantitative analysis of major elements on both untreated graphite substrate and the nanonetwork platform created at high fluence. 

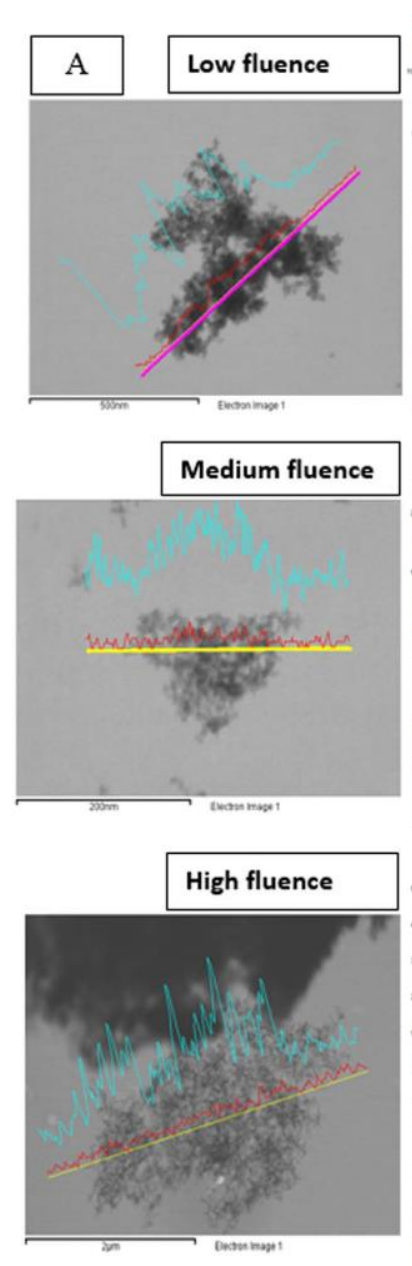
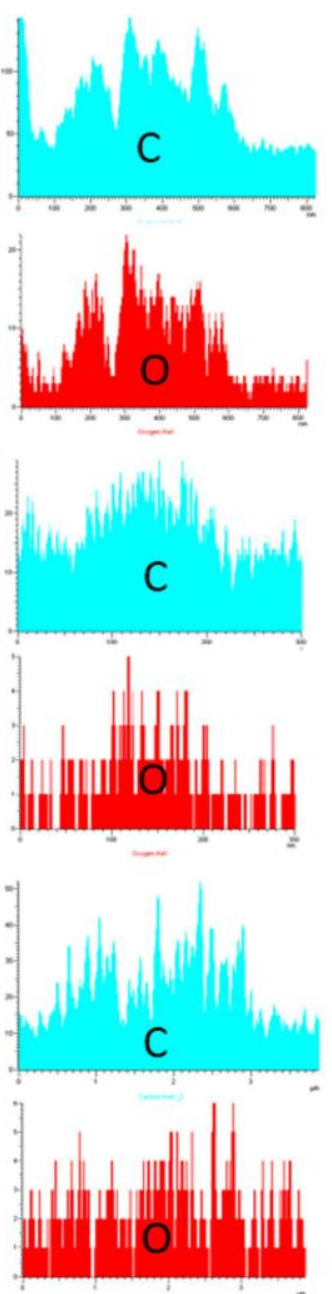

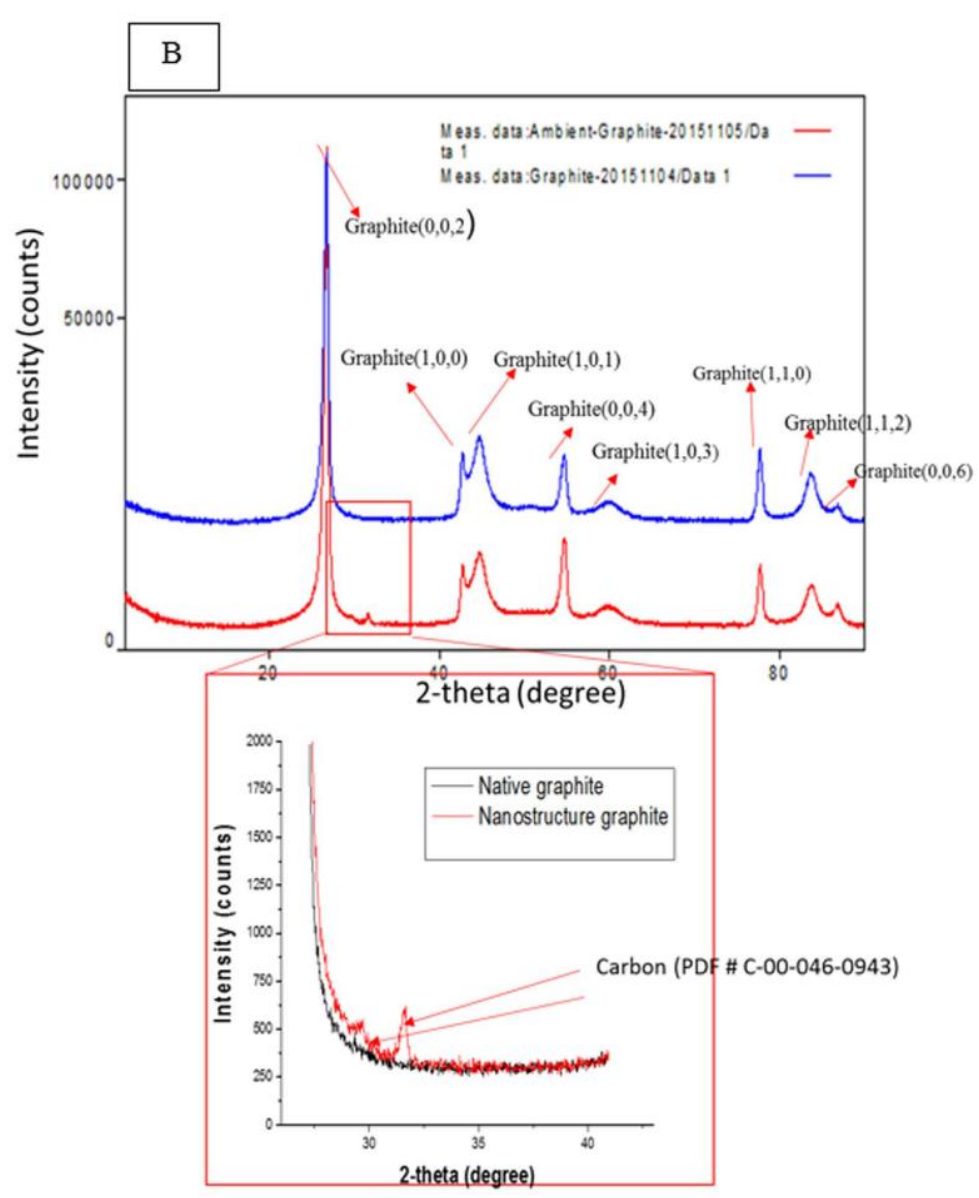

Figure 2-5: (A) FSEM-EDX elemental mapping of the created nano-network platforms at different laser fluences and their corresponding oxygen $(O)$ and Carbon $(C)$ spectra; (B) Comparison of XRD patterns of native graphite substrate and the nano-network platform indicating the presence of standard crystalline nano-graphite pattern as of native graphite except the peak at $2 \theta \approx 32$ which is associated to crystalline carbon (C-00-046-0943). 


\subsubsection{Cell interaction with carbon nano-network}

Fibroblast cells were used to study the cell adhesion to examine the biofunctionality of the synthesized 3-D bio-carbon nano-network platform. Fibroblasts were seeded on a native graphite substrate and 3-D bio-carbon nano-network platforms with low, medium, and high nano-network concentration, which were created on graphite substrates at different ultrashort pulse laser fluences, as illustrated in Fig.2-6.

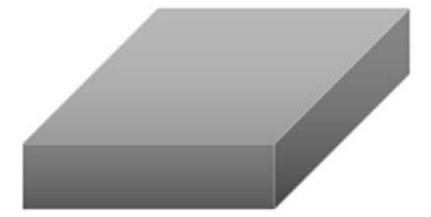

Native Graphite
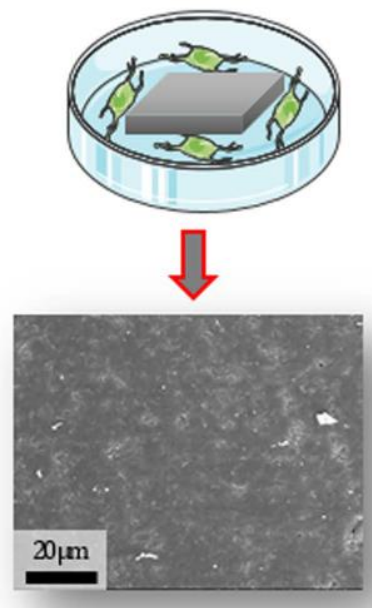
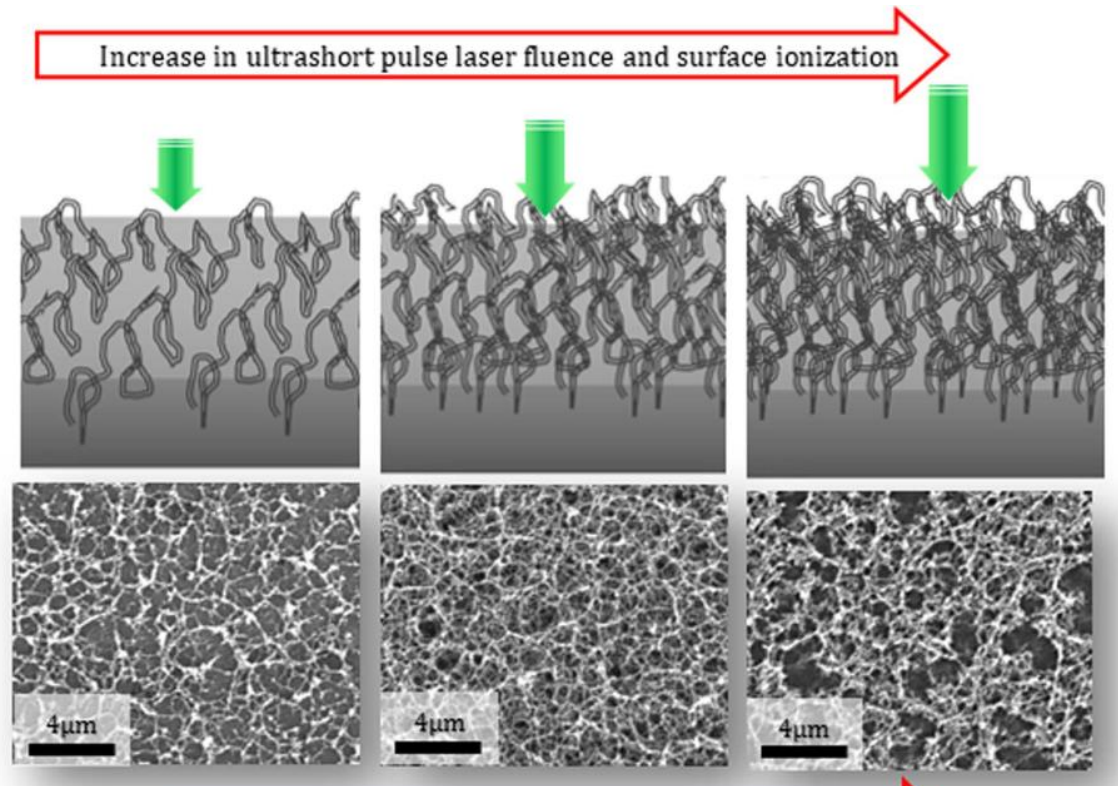

Increase in nano-network concentration
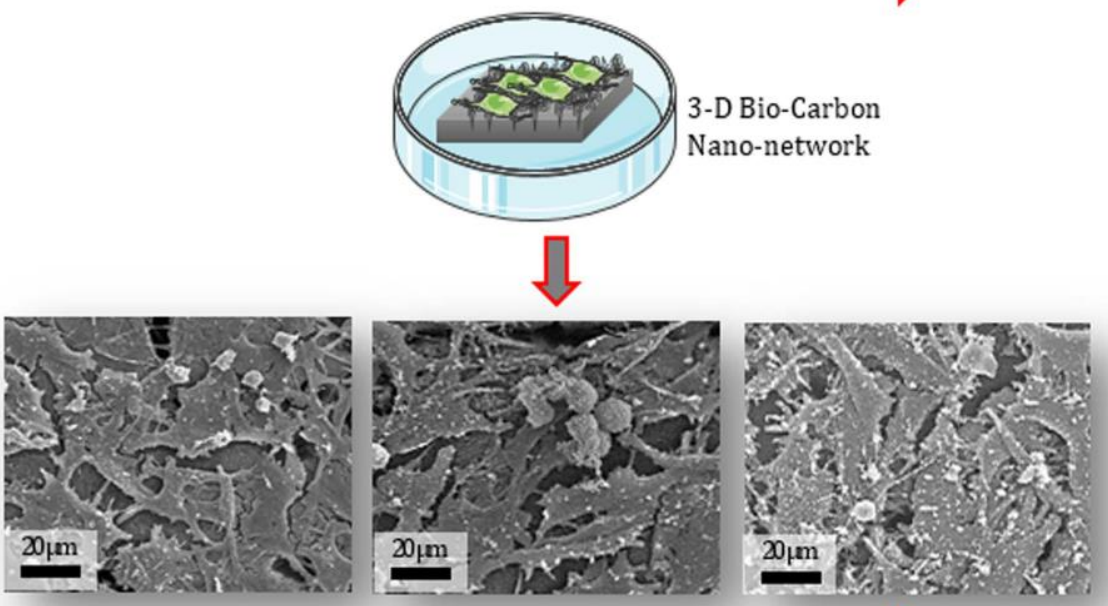

Increase in cell proliferation 
Figure 2-6: Schematic of native graphite and the nano-network platforms fabricated at different laser fluences and the corresponding SEM micrographs of the substrates before and after fibroblast response after $24 \mathrm{~h}$. (Graphics of the Petri dish and the cells were adapted from Servier Medical Art - creativecommons.org).

SEM (Fig.2-7(A-D) and (H-J)) micrographs of adhered cells were used to assess the cell adhesion and the cell morphological characteristics.

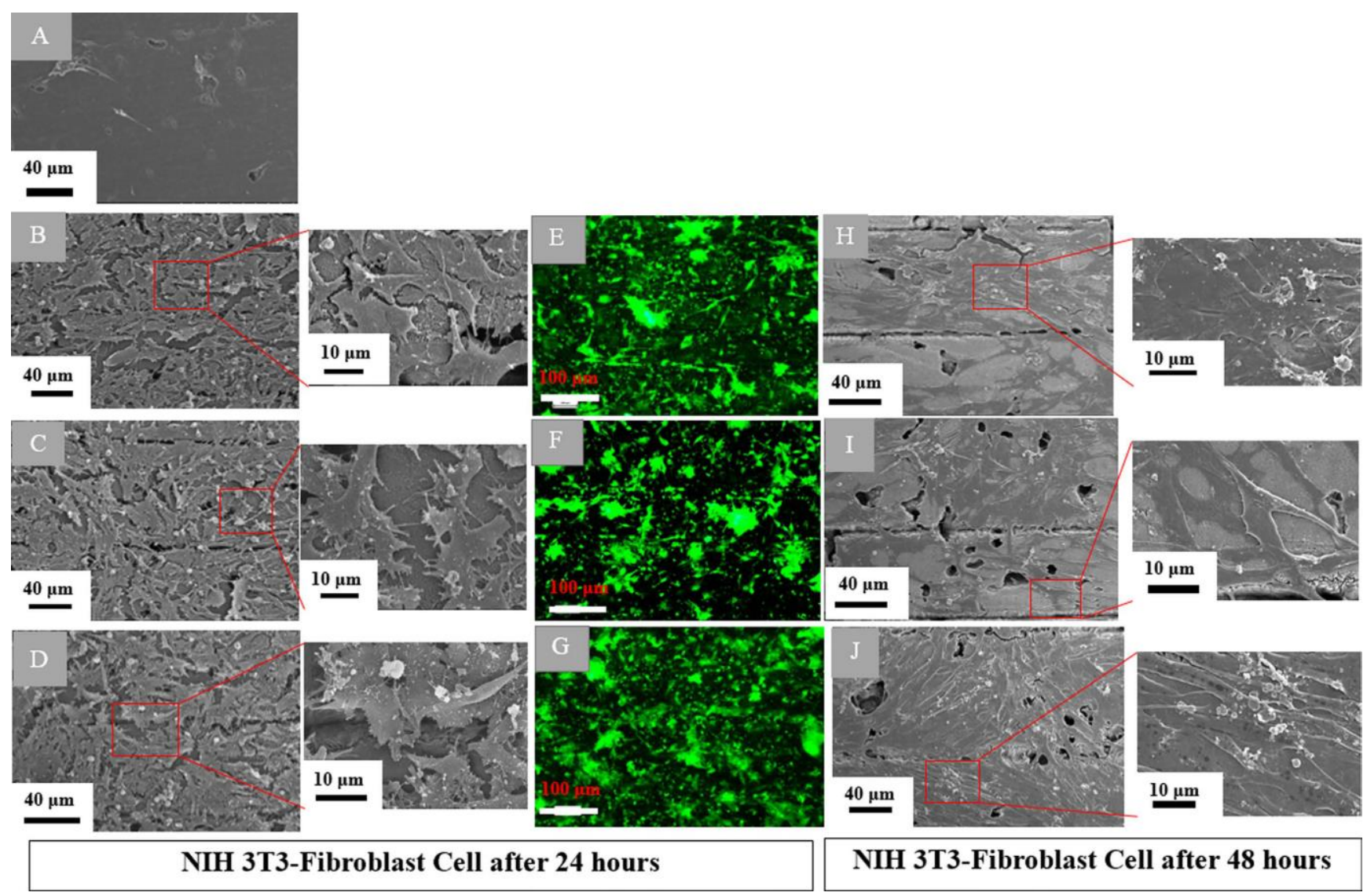

Figure 2-7: SEM micrographs of fibroblasts adhered on (A) native graphite and (B-D) the nanonetwork platforms created at low, medium and high fluences, respectively; corresponding fluorescence microscopy images of $(\mathrm{E}-\mathrm{G})$ nano-network platforms created at low, medium and high fluence, respectively after $24 \mathrm{~h}$ of culture; and SEM micrographs of fibroblasts cultured on (H-J) low, medium and high fluence mediated nanonetwork, respectively, after $48 \mathrm{~h}$. 
Studying the SEM micrographs of the native graphite substrate (Fig.2-7 (A)) revealed that no cell was attached to the substrate surface. Whereas, the fibroblasts cultured for $24 \mathrm{~h}$ on the 3-D biocarbon nano-network platform synthesized by low, medium and high ultrashort pulse laser fluence (Fig.2-7(B-D)) were attached perfectly. The number of attached cells varied on different substrates with a rising trend from low to high fluence. There was also a morphological symmetry of the cells among those adhered to nano-network. The cells were well adhered to the substrate, flats, proliferative, overlapping each other and covering the entire nano-network platforms for all the cases. The fibroblasts attached to the 3-D bio-carbon nano-network platform was well spread and flattened on the substrate with a morphological symmetry and their filopodia and actin filaments overextended along the nano-network zones, which is suggestive of high cellular interaction with a substrate. Cell area after $48 \mathrm{~h}$ of culture for cells adhered on nano-network platforms was significantly greater compared to that after $24 \mathrm{~h}$ in vitro. Fluorescence images (Fig.2-7(E-G)) were coherent with the SEM micrographs. According to the SEM micrographs of cells cultured for 48 h (Fig.2-7(H-J)), the density and size of the fibroblasts increased dramatically. The fibroblast attached to 3-D bio-carbon nano-network platform was organized intimately, sometimes overlapping each other. After $48 \mathrm{~h}$ of culture, the cells interlinked with each other and formed tissue-like structures. The trend is more prominent on the nano-network created at high laser fluence. In the next part of the study, we counted the number of cells that adhered on nano-network platforms fabricated at different ultrashort pulse laser fluences in comparison with the native control surface (see Fig.2-8). The number of cells attached to the native surface was negligible compared to that of the nano-network areas. Cell morphology could be a good indication of cell response to the substrates, e.g., well-spread polygon shape with extended filopodia is suggestive of healthy cells and increased cellular interaction with a substrate, and round shape is mainly 
indicative of non-proliferating/apoptotic like cells ${ }^{[2-35,2-36]}$. Therefore, we separately screened the number of attached flat cells and round cell to realize the proliferation of healthy cells on nanonetwork. As observed from the bar graph in Fig.2-8, there was an increasing trend in the number of flat cells adhered on the low fluence platform to high fluence one. While, the number of rounded cells showed a steady trend over different platforms.

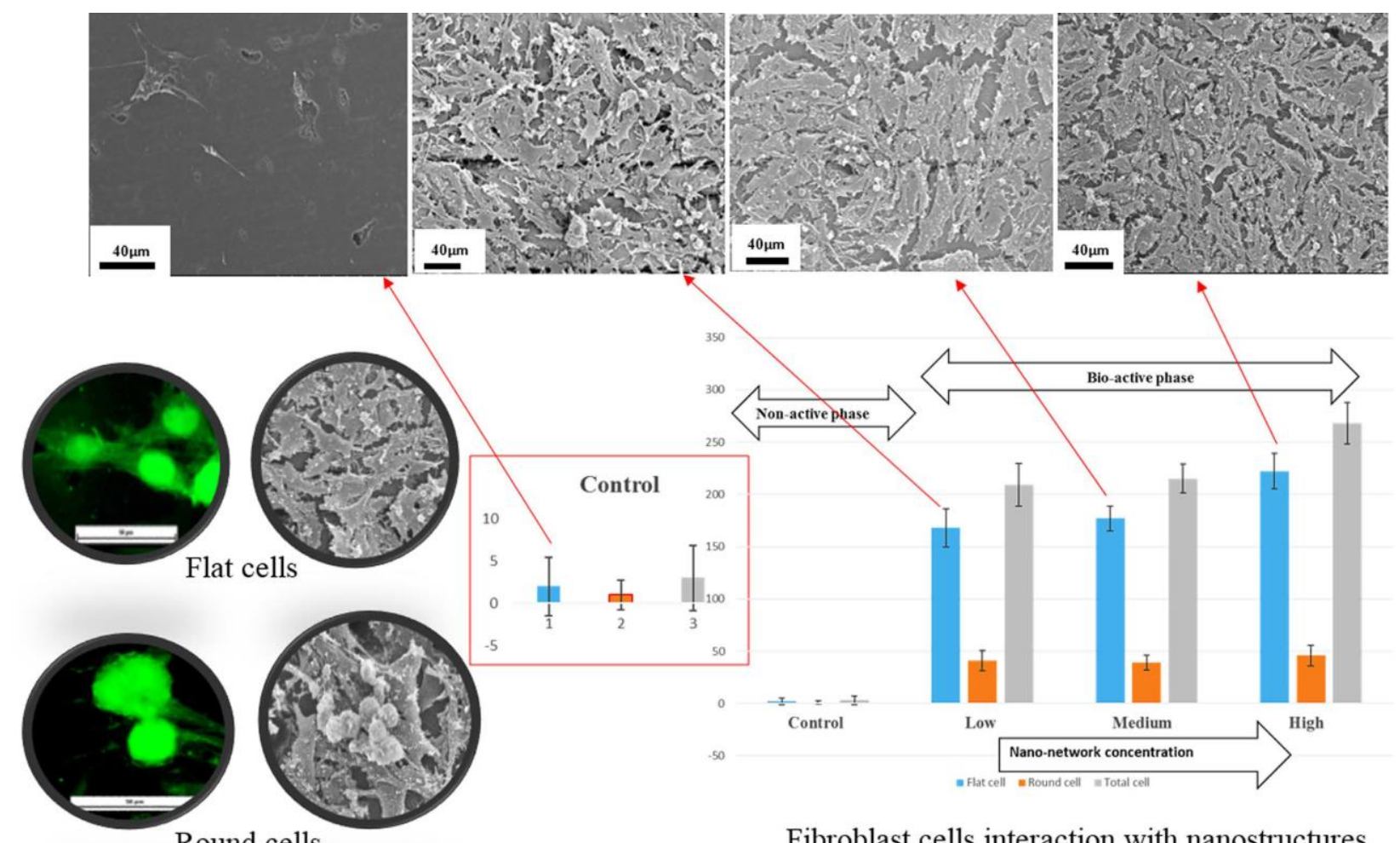

Figure2-8: Quantitative analysis of the number of NIH $3 \mathrm{~T} 3$ fibroblasts adhered on the untreated graphite substrate and the nano-network platforms created at different fluences.

SEM micrographs in Fig.2-9 depict the response of individual cells with carbon nano-network after 24 and $48 \mathrm{~h}$ of growth. The cell adhesion and spreading pattern were almost the same for all the nano-network areas created at different ultrashort pulse laser fluences. The cells were welladhered with flat shaped, and horizontally extended filopodia over the nano-network, which suggested strong adhesion. The filopodial extensions and anchoring points were shorter for the cells attached on low fluence mediated nano-network after $24 \mathrm{~h}$ and not prominent in $48 \mathrm{~h}$ (Fig.2- 
9(D)) compared to those adhered on high fluence platform. On the other hand, the long and strong filopodia extensions were observed for medium and high fluence mediated nano-network after both 24 and $48 \mathrm{~h}$.
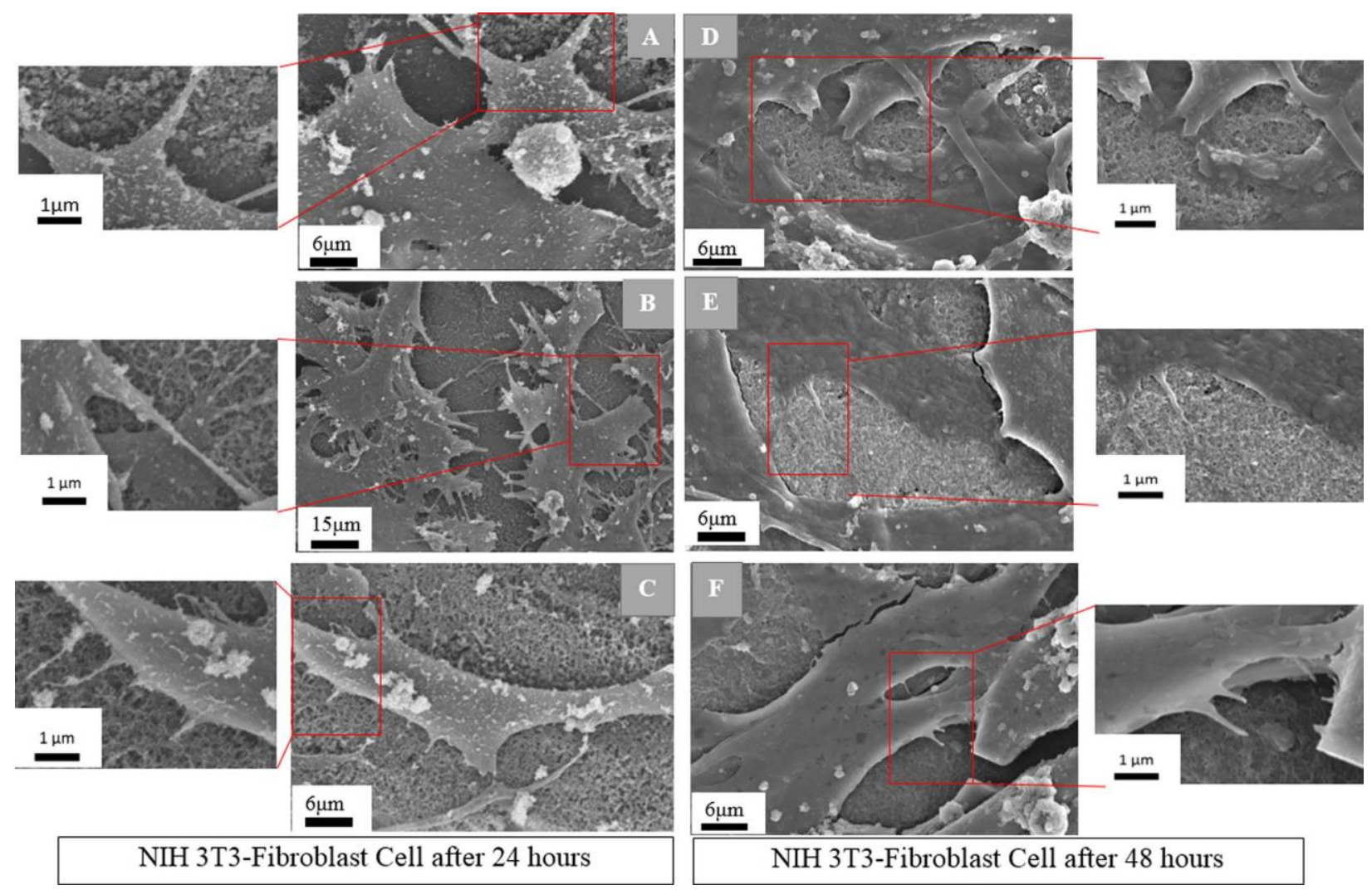

Figure 2-9: SEM micrographs of the fibroblast cells adhered on low, medium and high fluence mediated nanonetwork platforms, respectively, after (A-C) $24 \mathrm{~h}$ and (D-F) $48 \mathrm{~h}$ of culture. To further analyze cell phenotype and nucleus, fluorescence microscopy was employed where actin cytoskeleton and the nucleus of cells were stained in green and blue, respectively. The results were consistent with the SEM observations. The fluorescence data from Fig.2-10(A-C) revealed the activity of both cytoskeleton and nucleus of fibroblasts on nano-network synthesized under varying fluence. The fibroblast cells were nicely adhered and grew by spreading over the nanostructured area for all three fluence mediated nano-network. In comparison to low fluence mediated area, the cells were well spread and flat in the other two nano-network (Fig.2-10 (B, C). 
Contractile stress fibers, which have been shown to play an important role in cell adhesion and migration $^{[2-37]}$, were also observed for cells on all three platforms cultured both for 24 and $48 \mathrm{~h}$, as depicted in Fig.2-10. The area of nuclei of the cells for different platforms was also measured and

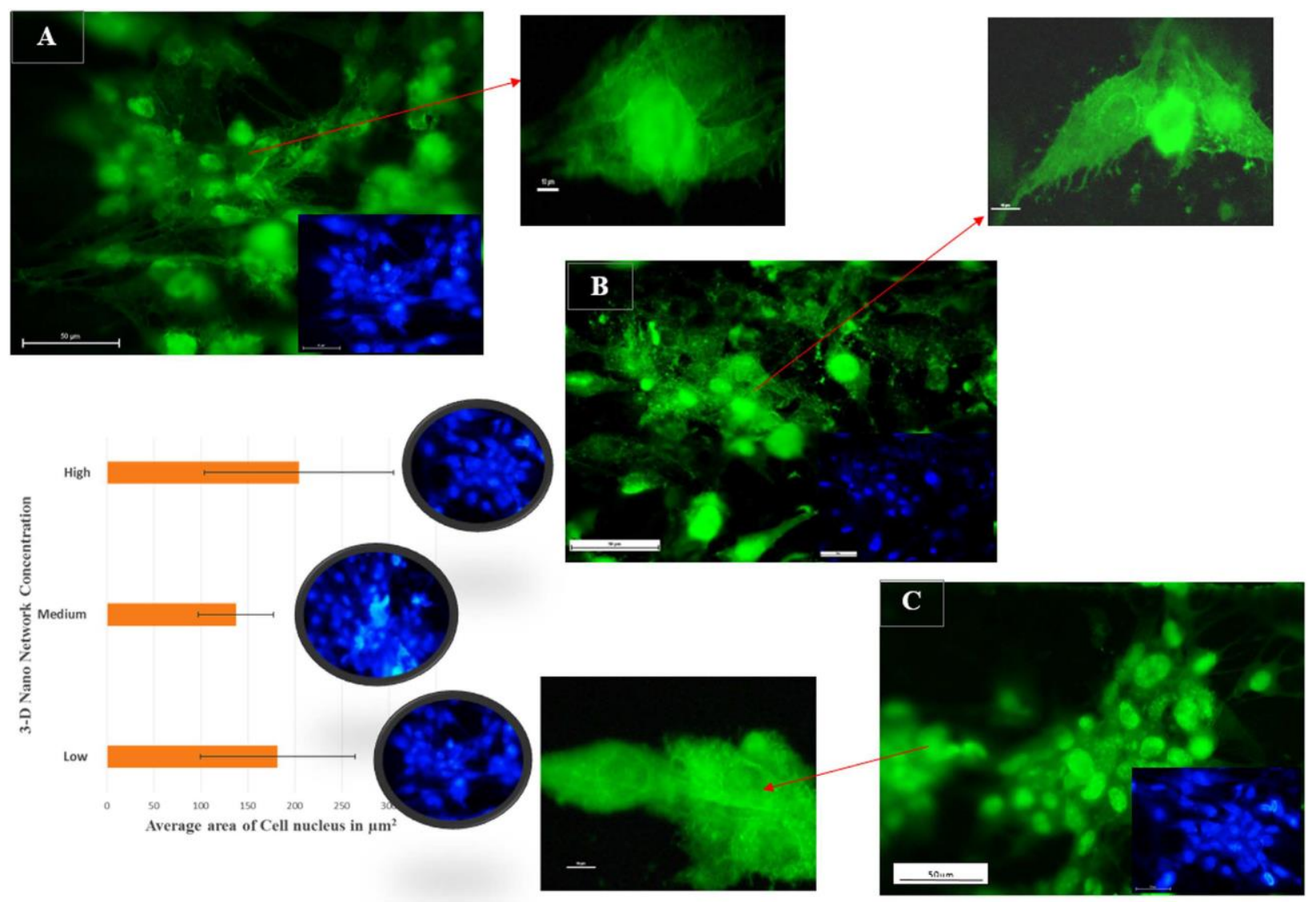

Figure 2-10: Fluorescence microscopy images and quantitative analysis of the surface area of the nucleus of fibroblasts attached on the platforms created at (A) low (B) medium, and (C) high laser fluence.

plotted in Fig.2-10 for comparing the size of the cells. Since, the cells adhered on the nano-network platforms grew very well and overlapped each other, distinguishing and counting an individual cell was deemed very difficult, and counting the area of cells might lead to inaccurate results. Therefore, the quantitative analysis of the area of the nuclei was performed instead to obtain the 
most accurate results and correlated those to the cell size, which were in agreement with qualitative results from SEM observation of cells. There is a direct relationship between cell size and nuclear size, and for a given cell type the nuclear/cell volume ratio (karyoplasmic ratio ${ }^{[2-38]}$ ) is said to be constant $^{[2-38,2-39,2-40]}$. Though there was a variation in the area of nucleus in the three platforms, the morphological character was the same for all the platforms.

\subsection{Discussions}

In this study, bio-functionalization of graphite surface were achieved through the synthesis of 3-D bio-carbon nano-network platform on graphite substrates by ultrashort femtosecond laser processing at ambient atmosphere. At the time of interaction between laser pulses with substrate surface on femtosecond time scale, energy deposited into the material faster than needed for the system to react and lead to ion ejection, i.e. ablation from the target. Unlike nanosecond/picosecond laser ablation, femtosecond laser processing has the advantage of having little or no collateral damage due to shock waves and heat conduction produced in the material being processed ${ }^{[2-41]}$. The primary mechanism leading to ablation by femtosecond laser pulses were mechanical fragmentation, homogeneous nucleation and vaporization ${ }^{[2-42]}$. A high temperature reactive multimodal ablation plasma plume containing neutral carbon $(\mathrm{C})$, carbon radicals $\left(\mathrm{C}_{2}, \mathrm{C}_{3}\right)$ and carbon ions $\left(\mathrm{C}^{+}, \mathrm{C}^{2+}\right)$ was generated due to the femtosecond laser irradiation of graphite substrate ${ }^{[2-43]}$. The faster-moving carbon ions situate at the top of the expanding plume while heavier radicals and neutral carbon molecules reside at the bottom. The change of fluence from low to high controls the ratios of the plume components and has a significant effect on the growth of nanofibers. With the increment of ultrashort pulse laser fluence, more energy is induced into the plume, which in turn enhances the ratio of carbon ion to other components in the plasma plume. This results in higher level of carbon ionization $\left(\mathrm{C}^{+}, \mathrm{C}^{2+}\right)$ and increases the number of 
carbon nano-species in the plasma plume, which will eventually condense and aggregate to form densely packed interwoven nano-network. There was an ascending trend in web-like interlinked 3-D nano-network created in and around to laser irradiated lines with the increase in ultrashort pulse laser fluence due to the indicated reasons, which was evident from the SEM micrographs of the substrates (Fig.2-2(B-D)). When plasma plume got outward expansion in presence of ambient atmospheric gas, there was a heat transfer between them and subsequent condensation took place. The oxygen atom that present in the ambient gas and came in contact with the boundary of the plume had a chemical interaction with the carbon ion and there was some trace of $\mathrm{C}-\mathrm{O}$ molecular bonding presence in the created nano-network, as evidenced by the FE-SEM-EDX analysis in Fig. 2-5(A). Micro-Raman spectra of both native graphite substrate as well as the nano-network areas (Fig.2-3(A)) showed the graphite specific spectrum with graphite G- Peak and 'disorder induced' D peak as referred to elsewhere ${ }^{[2-44]}$, although there was a slight deviation of positions of the peaks. The graphite crystallite size $L a$ gave a focus on the characteristic changes by femtosecond irradiation during recrystallization process, as shown in Fig.2-3(B). The nanocrystallite size of the nano-network formed reduces with the increase of laser fluence. The high fluence with more ionization energy resulted in more carbon ions in the plume, which when condensed created widespread nano-network branches of reduced crystallite size, evidenced from the FE-SEM (Fig.22(D)). Several factors, such as material physical properties, degree of ionization, and the ambient gas characteristics, contribute a remarkable influence on the crystalline structure of the products after laser ablation ${ }^{[2-41,2-45]}$. However, since in this study laser ablation is performed in ambient air at atmospheric pressure, the plume expansion, condensation, nucleation, and crystallization are mostly governed by diffusion to the background air rather than adiabatic expansion as in laser ablation in vacuum. Therefore, the characteristics of ambient gas play a much more important role 
on the crystalline structure of the nanostructures than the degree of ionization/plume temperature, which are slightly affected by laser fluence ${ }^{[2-41]}$. This is why the changes in crystal size was only about $10 \%$. Our results from micro-Raman and XPS analyses revealed that there were changes in surface chemistry due to the presence of $\mathrm{C}-\mathrm{O}$ molecular bond along with the $\mathrm{C}-\mathrm{C}$ bonds in the created nano-network. Surface of carbon nano-network platform had higher amount of oxygen in comparison with native substrate, which was the result of surface ionization at very high temperature in ambient atmosphere. As our findings showed, the nano-network carbon consisted of crystalline carbon with larger crystalline size compared to native graphite. XRD analysis also confirmed the dominance of $\mathrm{C}-\mathrm{C}$ bonds in the resulted 3-D bio-carbon nano-network platform with the presence of standard nano-graphite crystalline orientations which was in line with standard graphite diffraction pattern.

Our studies on fibroblast cell response to the synthesized 3-D bio-carbon nano-network platform showed that the interwoven nano-network were effectively affirmative in adhering the cells compared to the native graphite surface where the same cells were seeded for $24 \mathrm{~h}$. The native graphite surface has failed to generate enough favorable cues for initial attachment of the fibroblast cells. According to recent studies, the adhesion of cells to a substrate can be divided into two basic steps: initial attachment and adhesion ${ }^{[2-46]}$. The change in surface nanotopography resulted from 3-D nano-network platform and surface chemistry because of the presence of $\mathrm{C}-\mathrm{O}$ bond in the nano-network provided stimulation for cell adhesion. The resulted nano-network surface is believed to improve adsorption of different extracellular matrix proteins such as fibronectin, vitronectin and collagen, which ultimately helps cell adhesion ${ }^{[2-47]}$. These molecules might be adsorbed from the serum of the culture medium in such an appropriate amount with favorable conformation that cell adhesion receptors, e.g., integrins, are able to access specific amino acid 
sequences for affirmative cell adhesion ${ }^{[2-48,2-49,2-50]}$. Again, in the biological interactions of nanomaterials, surface area plays a key role ${ }^{[2-51,2-52]}$. The bio-carbon nano-network platform synthesized at high fluence posse greater degree of interconnected nanofibers, which results in a higher surface area with reduced crystallite size compare to that of low fluence-mediated nanonetwork. This might give a significant amount of surface exposure for protein adsorption and eventually more sites for cells adhesion ${ }^{[2-53]}$. The extracellular matrix (ECM)-like morphology of the nano-network, which provides the tissue-like micro-environment for the cells, promote subsequent cell adhesion and proliferation. In order to evaluate the biocompatibility of bio-carbon nano-network platform, a quantitative analysis of the number of NIH 3T3 fibroblast cell adhered on nano-network platforms at different fluence conditions (low, medium and high) was performed (Fig.2-8). The cells adhered on the nano-network areas were flat and well adhered. The number of rounded cells was very less compare to the flat cells. Normal morphological characteristics of flat cells with well-formed cytoskeleton and filopodia indicated the biocompatibility of bio-carbon nano-network platform. Also, the fact that all the cell that adhered on the nano-network platform synthesized with low, medium and high fluence formed stress fibers, as evident from Fig.2-10, implied that the resulted nano-network provided favorable cues for initiating stress fibers. On the other hand, the rounded cells that were counted mostly resided on the top of the other flat cells and in agglomerated form. These cells might not have found a proper site or contact to start proliferation. Therefore, the findings suggested the positive proliferative nature of the developed nano-network platform and confirmed that it promoted robust fibroblast growth. It is expected that the proposed approach to bio-functionalize carbon surface advance the development of new biomedical devices for diverse biomedical applications, such as tissue engineering, orthopedic and dental implants, and drug delivery devices. 


\subsection{Summary}

In this work, we reported the developing a new bio-carbon nano-network platform with significant proliferative property on fibroblast in a single step without the use of post-biological or -chemical treatments. We applied a bottom-up approach based on ultrafast laser ionization to biofunctionalize graphite substrate via the creation of self-assembled 3-D carbon nano-networks. Ultrafast laser ionization of graphite resulted in graphite ionization and generation of 3-D biocarbon nano-network platform with modified nano-chemistry and nanotopography. Different degrees of cell proliferations were achieved by controlling the transferred ultrashort pulse laser energy to the surface, which directly altered both the concentration and nano-chemistry of the nano-network. The in vitro biocompatibility of the fabricated nano-network was studied quantitatively and qualitatively with fibroblasts. The findings from SEM and FM analyses confirmed strong attachment and growth of healthy fibroblast cells on the graphitic carbon nanonetwork compared to the untreated counterpart. The cell morphology and adhesion patterns were nearly the same for all the nano-network areas fabricated at different ultrashort pulse laser fluences. The number of cells adhered on the high fluence-fabricated platform in $24 \mathrm{~h}$ was 91 -fold more compared to untreated graphite, which indicated the high proliferative nature of the developed nano-network. Interlinked tissue-like structures were observed after $48 \mathrm{~h}$ of culture, where no single cell was clearly identifiable. The results of this study suggest that the developed nanonetwork platform not only is biocompatible but also encourages fibroblast proliferation vigorously. The simplicity of the proposed technique to rapidly transform biologically-inactive graphite to biocompatible cell-adhesive substrates will make it a unique approach for developing biofunctionalized carbon-based platforms for the use in biosensing, bioimaging, cancer therapy, and tissue engineering. 


\section{Chapter 3}

\section{Fibroblast-Cytophilic and HeLa-Cytotoxic Dual Function Carbon Nanoribbon Network Platform}

Published in ACS Applied Materials and Interface, May 2017

Carbon nanomaterials have emerged as a promising material in cancer diagnosis and therapy. Carbon nanomaterials/nanostructures (C-C molecular structure) act as a carrier/skeleton and require further surface modification through functionalization with chemicals or biomolecules to attain a cell response. We report the synthesis of a novel carbon nanoribbon network (CNRN) platform that possesses a combination of $\mathrm{C}-\mathrm{C}$ and $\mathrm{C}-\mathrm{O}$ bond architecture. The bioactive CNRN showed enhanced ability for cell adhesion. Most importantly, it induced opposite cell responses from healthy cells and cancerous cells, cytophilic to fibroblasts but cytotoxic to HeLa cells. Ultrafast laser ionization in ambient conditions transforms nonbioresponsive C-C bond of graphite to $\mathrm{C}-\mathrm{C}$ and $\mathrm{C}-\mathrm{O}$ bonds, forming self-assembled CNRN platform. The morphology, nanochemistry and functionality on modulating fibroblast and HeLa adhesion and proliferation of the fabricated CNRN platforms were investigated. The results of in vitro studies suggested that the CNRN platforms not only attracted but also actively accelerated the adhesion and proliferation of both fibroblasts and HeLa cells. The proliferation rate of fibroblasts and HeLa cells is 91 and 98 times greater compared with that of a native graphite substrate, respectively. The morphology of the cells over a period of 24 to $48 \mathrm{~h}$ revealed that the CNRN platform induced an apoptosis-like cytotoxic function on HeLa cells, whereas fibroblasts experienced a cytophilic effect and formed a tissuelike structure. The degree of cytotoxic or cytophilic effect can be further enhanced by adjusting parameters such as the ratio of $\mathrm{C}-\mathrm{C}$ bonds to $\mathrm{C}-\mathrm{O}$ bonds, the nanoribbon width, and the nanovoid 
porosity of the CNRN platforms, which could be tuned by careful control of laser ionization. In a nutshell, for the first-time pristine carbon nanostructure free from biochemical functionalization demonstrate dual function, cytophilic to fibroblast cells and cytotoxic to HeLa cells.

\subsection{Introduction}

Nanomaterials with a proven track record in biomaterial research have shown substantial promise in cancer diagnosis and therapy. Carbon-based nanomaterials, such as carbon nanotubes (CNTs), ${ }^{[3-1]}$ carbon nanowalls $(\mathrm{CNW}),{ }^{[3-2]}$ and graphene and its derivatives, ${ }^{[3-3,3-4]}$ have been actively explored for cancer research, which includes early detection of cancer, ${ }^{[3-5]}$ chemo-photothermal therapy, ${ }^{[3-6]}$ and controlled drug delivery. ${ }^{[3-7]}$ When used as an agent for cancer treatment and diagnosis, ${ }^{[3-1,3-8]} \mathrm{CNTs}$ act as nanocarriers loaded with a variety of biomolecules and drugs. CNWs provide a skeleton for the conjugation of chemical molecules that help in the attachment of cells. The current studies of CNWs for cell adhesion and proliferation behavior, mammalian or cancer, have been limited to chemical surface alterations of CNWs through surface plasma functionalization. ${ }^{[3-2,3-9,3-10]}$

Pristine CNTs are generally cytotoxic. As-synthesized CNTs contain amorphous carbon and residue catalytic particles. Postsynthesis purification cannot completely remove these impurities. The cytotoxicity of these impurities can influence cell culture efficacy. ${ }^{[3-11]}$ As a result, pristine CNTs are seldom used alone as an anticancer agent. Hence, there are limited reports on the direct interaction of CNTs with cancer cells. Zhang et al. ${ }^{[3-12]}$ prepared five types of CNT scaffolds and investigated their influence on the viability of HeLa cells. They concluded that the viability of HeLa cells cultured on these scaffolds decreased greatly on single-wall carbon nanotubes (SWCNTs) compared with amylose-wrapped SWCNTs. 
Graphene is another branch of carbon nanomaterials that has gained much attention for cancer diagnosis and treatment. It has aided in the focal adhesion and proliferation of mammalian cell lines, such as fibroblasts and osteoblasts, with improved gene efficiency, without showing any toxic effects. ${ }^{[3-13,3-14]}$ On the other hand, some researchers reported the induction of oxidative stress in cultured cells because of the formation of reactive oxygen species on the graphene surface $[3-15,3-16]$. The cellular interactions, specifically the cellular proliferation, spreading area, and cytotoxicity, of graphene and its derivatives with different cancer cell lines have also been actively investigated by different research groups. ${ }^{[3-17,3-18,3-19]}$ Markovic et al. ${ }^{[3-20]}$ with their experiment on the photothermal anticancer activity of near infrared (NIR)-excited graphene suggested that graphene nanoparticles performed significantly better than CNTs in inducing the photothermal death of human glioma (U251) cells in vitro. However, it requires an external source to induce anticancer activity.

Graphite exists as a layered material in its bulk form. Exfoliation is usually employed to modify the surface of graphite to produce graphene. ${ }^{[3-21]}$ A variety of physiochemical strategies have been developed for graphite exfoliation. Throckmorton and Palmese ${ }^{[3-22]}$ presented an alternative path for direct exfoliation of graphite, which is capable of single-pot preparation of nanocomposites of graphene. Bai et al. ${ }^{[3-23]}$ developed a sonochemical approach and synthesized graphene- gold nanocomposites from graphite flakes. Regardless of synthesis routes, similar to CNTs, carbon nanomaterials/ nanostructures with $\mathrm{C}-\mathrm{C}$ bonds as their core structure have primarily acted as carriers/skeletons and have required further functionalization to attain biocompatibility and cell response. The multistep functionalization conjugates therapeutic molecules or ligands onto the graphene surface to render its anticancer property. 


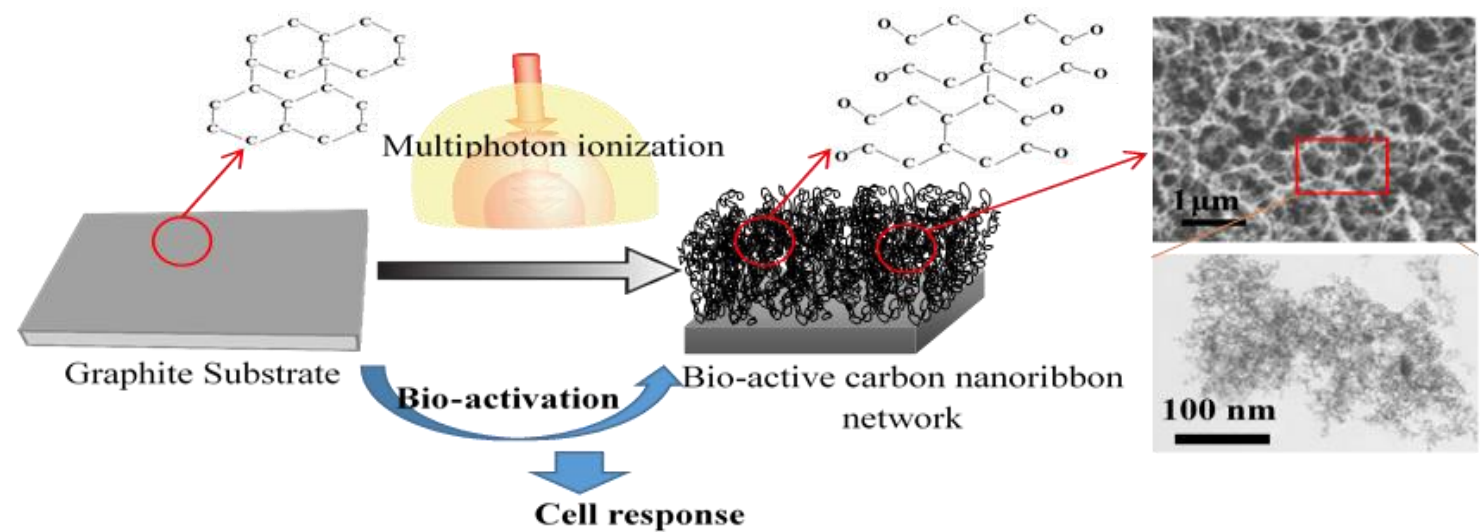

Before bio-activation

After bio-activation

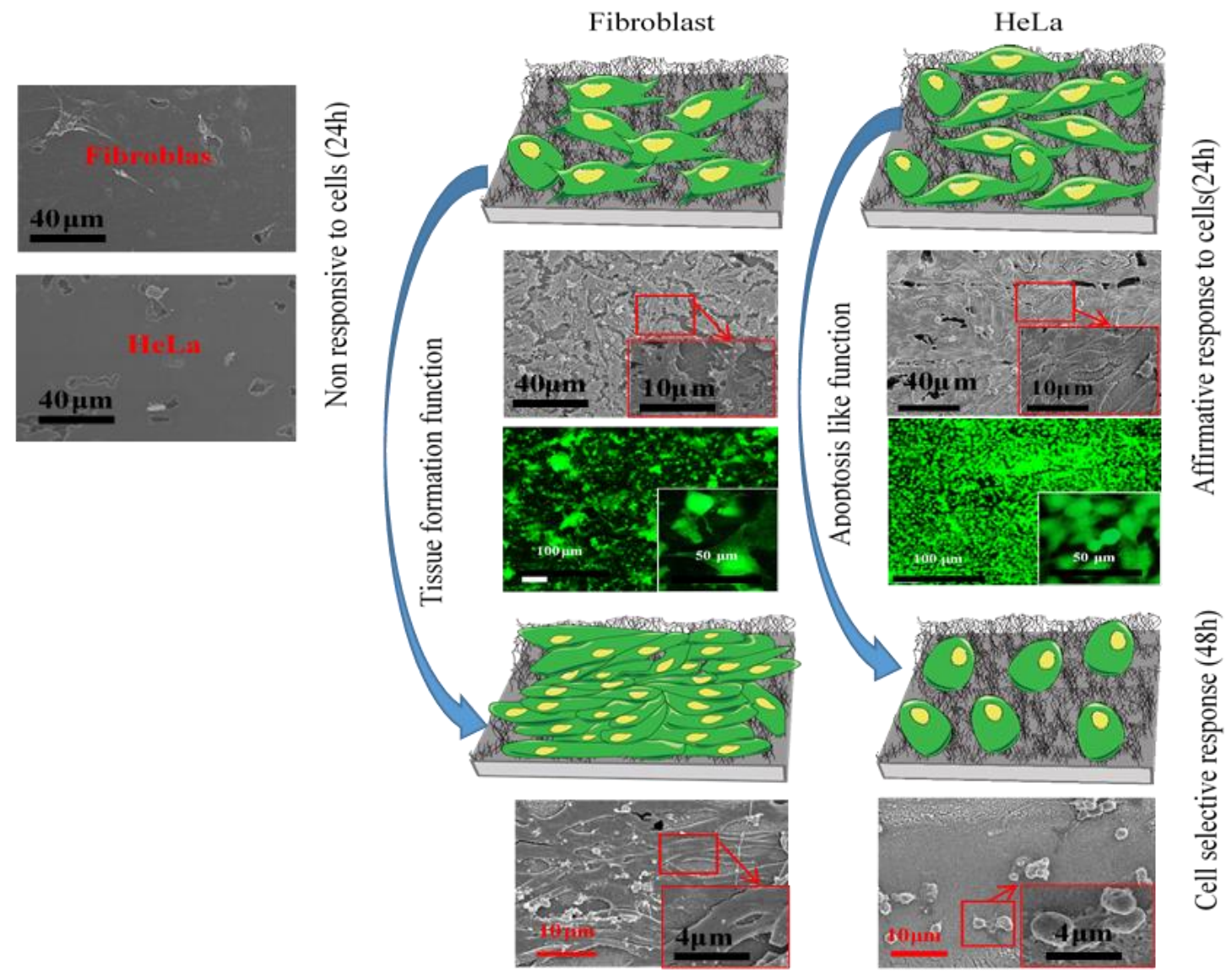

Figure 3-1: Graphical abstract showing bio activation of carbon nanoribbon network with multiphoton ionization and time dependent fibroblast and HeLa cell response on the created carbon nanoribbon network. 
In the present investigation, we report the synthesis of a unique carbon nanoribbon network $(\mathrm{CNRN})$ platform that comprises a mixture of $\mathrm{C}-\mathrm{C}$ and $\mathrm{C}-\mathrm{O}$ molecular bonds. The platform was developed by adopting a self-assembled bottomup approach using femtosecond laser ionization that converted the non-bioresponsive graphite substrates to a bioactive interwoven CNRN platform. This process modified both the nanotopography and the nanochemistry of the carbon structure. The adjustment of laser ionization parameters resulted in variations in physical morphology, such as the nanoribbon width and nanovoid density, and in chemical composition, such as the ratio of the $\mathrm{C}-\mathrm{O}$ bond, which plays a major role in creating variance in attracting, adhering, and proliferating both fibroblast and HeLa cells. Initially, the created nanoribbon network acts as a favorable attractor and a proliferation-promoting substrate for both fibroblasts and HeLa cells. In time, the same platform acted more favorable for the proliferation of fibroblasts but apoptosis for HeLa cells. In other words, the CNRN platform induced cell-specific anticancer cytotoxicity with the passage of time with no harm on mammalian cells. These results indicate the possibility of pristine carbon nanostructure as an anticancer agent with minimal side effects.

\subsection{Experimental Method}

\subsubsection{Bioactive CNRN Platform Fabrication.}

Square samples $(2 \times 2 \mathrm{~cm})$ were cut from $3 \mathrm{~mm}$ thick isomolded graphite plates (Graphtek LLC, USA) with extremely fine grain and high strength. The surface of the samples was polished with sand paper (3M Canada, 1000, 2000, and 3000 grit) and then ultrasonically cleaned (Cole-Parmer 8890 ultrasonic cleaner) with acetone and ethanol for $5 \mathrm{~min}$, followed by ringing and drying at room temperature. Multiphoton ionization was created with a diode-pump, an Yb-doped femtosecond laser system (Clark-MXR, Inc.; IMPULSE Series ultrashort pulse laser), to synthesize the bioactive CNRN platform on the top polished surface of the square graphite 
substrate plate. Three different laser pulse repetitions of 4, 8, and $26 \mathrm{MHz}$ and two laser beam scanning speeds of 1 and $7.5 \mathrm{~mm} / \mathrm{s}$ under atmospheric conditions were used to alter the nanotopography and nanochemistry of the developed nanonetwork. The pulse energy transferred to the substrates per unit area (ultrashort pulse laser fluence) was varied at 4.43, 2.22, and 0.68 $\mathrm{J} / \mathrm{cm}^{2}$, which are referred to as high, medium, and low influence throughout this study. The sample was mounted on a fixed stage, and a computer-controlled high-precision two-dimensional (2D) translation scanner guided the incident laser beam. To create the bioactive CNRN platform, an array of lines with variable line spacing was ablated. For all laser pulses, the laser pulse width, the power of the incident laser beam, and the irradiation focal spot area were maintained constant at $214 \mathrm{fs}, 15 \mathrm{~W}$, and $84.62 \times 10^{-8} \mathrm{~cm}^{2}$, respectively. A computer-based monitoring system assured the consistency and accuracy of these parameters.

\subsubsection{Morphological and Physicochemical Characterization of the CNRN Platform.}

Scanning electron microscopy (SEM) (Hitachi SU-1500) was employed to describe the surface morphology of the self-assembled bioactive CNRN platform. Field emission SEM (FESEM, Hitachi, SU-8200) was used to determine the nanoribbon width distribution and nanovoid area distribution for each created CNRN platform. Analysis of the images from FE-SEM, using Image J (image processing software), revealed three categorizations of low, medium, and high dense structural characteristic indices.

Micro-Raman spectroscopy and X-ray photoelectron spectroscopy (XPS) were used to analyze the chemical composition of the bioactive CNRN platform. Micro-Raman spectroscopy also deduced crystallinity information of the created CNRN platform. A Bruker SENTERRA dispersive Raman Microscope (laser excitation wavelength was $532 \mathrm{~nm}$ ) was used to get the Raman spectra of the CNRN platform. 
To get the XPS data, $1 \mathrm{~cm}^{2}$ samples were irradiated with the laser system. The XPS data were collected using a Thermo Fisher K-Alpha XPS system using a monochromated Al Ka X-ray source with a 2:1 ellipse spot size of $400 \mathrm{~mm}$ as the major axis. Regional scans were performed at $50 \mathrm{eV}$ pass energy and $0.1 \mathrm{eV}$ point spacing to give better energy resolution and peak shape definition. Using the Avantage software, the quantification of $\mathrm{C}$ and $\mathrm{O}$ was obtained.

\subsubsection{Cell Culture.}

The functional assessment of the fabricated bioactive CNRN platform was accomplished using mouse embryonic fibroblasts (NIH-3T3, ATCC, USA) and cervical cancer cells (HeLa, ATCC, USA). These cells were grown in Dulbecco's modified Eagle's medium (DMEM)/F12 supplemented with $10 \%$ fetal bovine serum (FBS) and 1\% penicillin/streptomycin, and the cultures were incubated at $37{ }^{\circ} \mathrm{C}$ in a humidified $5 \% \mathrm{CO}_{2}-95 \%$ air atmosphere.

\subsubsection{NIH-3T3 Mouse Fibroblast Cells and Cervical Cancer Cells (HeLa) seeding on CNRN}

\section{Platform.}

Prior to in vitro studies, CNRN platforms were exposed to UV light for $20 \mathrm{~min}$ for sterilization. Subsequently, the platforms were placed inside Petri dishes containing $3 \mathrm{~mL}$ of DMEM/F12 medium and $10 \%$ FBS per dish, and the cells were seeded at a density of $10^{5}$ cells $/ \mathrm{mL}$. The Petri dishes were placed in an incubator for 24 or $48 \mathrm{~h}$. The samples were fixed with $2 \%$ glutaraldehyde after removing the spent medium. Subsequently, the samples were washed twice with $1 \%$ sodium cacodylate buffer $(\mathrm{pH} 7.3)$ at $4{ }^{\circ} \mathrm{C}$. Then, the cells were dehydrated through a graded ethanol series (from 10 to $100 \%$ ) for 15 min and were critical point dried. The samples were then sputtered with a gold layer before SEM examination. With a Hitachi SU1510 scanning electron microscope, the cellular morphology of the cells seeded on the samples was observed. The energy-dispersive X- 
ray (EDX) mapping of these samples was carried out using Oxford EDX to characterize CNRN uptakes caused by endocytosis.

\subsubsection{Fluorescent Staining of Cells.}

To perform fluorescence microscopy (FM), the samples were first fixed in methanol-free paraformaldehyde. Then, incubation in milk was performed to prevent nonspecific binding. Further incubation of the samples was then performed using Alexa Fluor 488 phalloidin (Life Technologies) to stain the actin and the cytoskeleton, followed by 4',6'-diamidino-2- phenylindole (DAPI, Life Technologies) to stain the nucleus. The samples were studied using an epifluorescent Nikon E-400 microscope with a fluorescein isothiocyanate (FITC) and DAPI filter. The data were recorded using a DS-5M-U1 color digital camera (Nikon, Canada).

\subsubsection{Statistics.}

All experiments were carried out in triplicate, and the data represented the mean \pm standard deviation, unless otherwise mentioned. The cell counting was carried out using SEM images and image processing software. One-way analysis of variance (ANOVA) was used to evaluate the statistical significance, with $* p<0.05$ and $* * p<0.01$ suggesting significant difference.

\subsection{Results}

\subsubsection{Fabrication and Characterization of the CNRN Platform.}

The bioactive CNRN platforms were synthesized by multiphoton ionization induced by one-step femtosecond laser processing on the surface of a graphite plate under atmospheric conditions, as illustrated in the schematic in Figure 3-2. A predesigned array of lines was transferred onto the surface of the graphite sample with $2 \mathrm{D}(\mathrm{X}-\mathrm{Y})$ movement of laser beam that was precisely controlled by a computerized Galvano scanner. Ultrashort laser processing of the graphite plate 


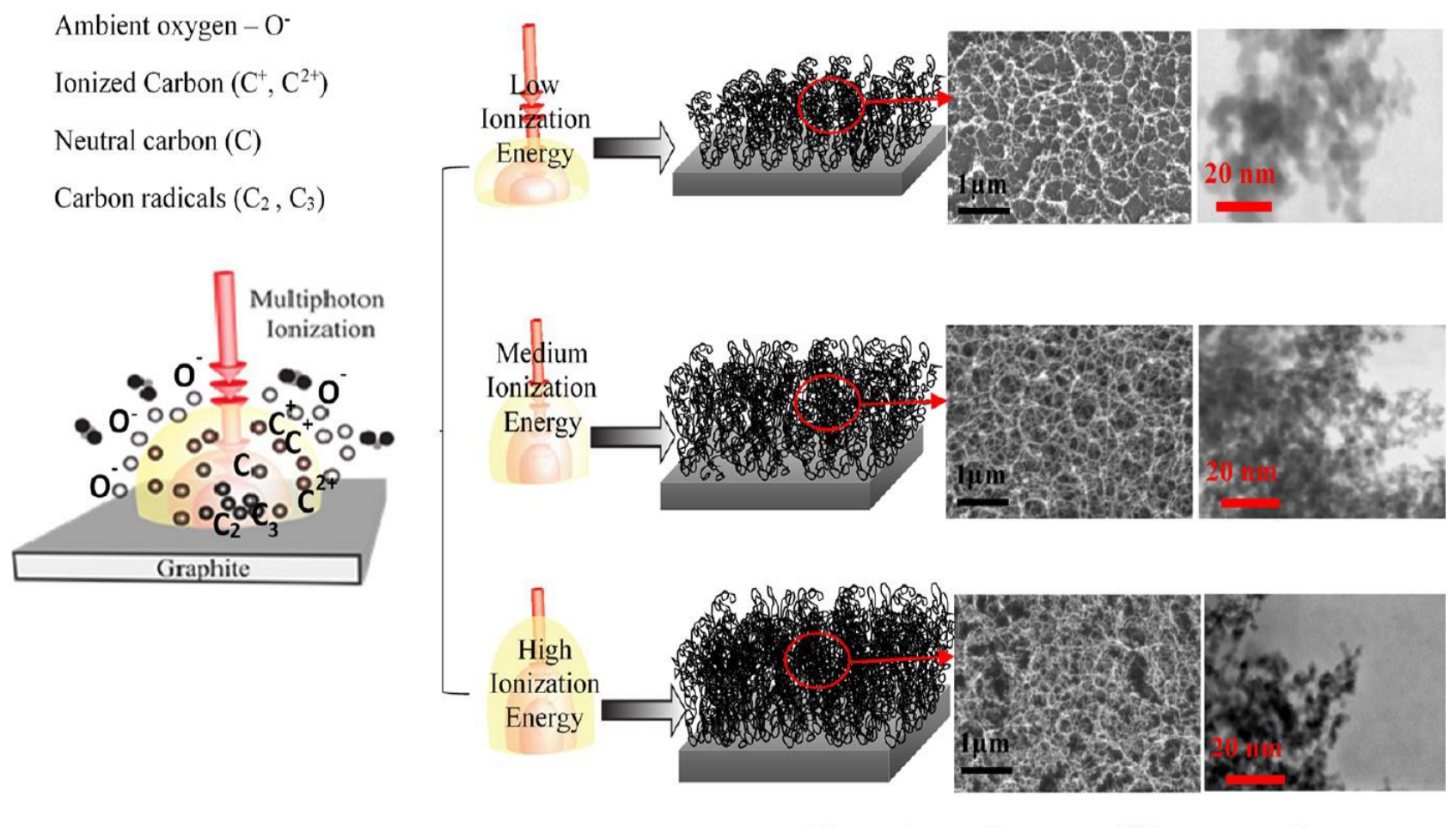

Bio active carbon nanoribbon network

Figure 3-2: Schematic illustration showing the single-step synthesis of the CNRN platform. Change in ionization energy precisely modulated the physical morphology and the $\mathrm{C}-\mathrm{O}$ bond percentage of the CNRN platform. using a megahertz pulse repetition rate resulted in surface ablation, nanoparticle evaporation, and formation of the vapor plume.

The dense vapor plume comprised different forms of carbon (carbon ions, carbon radicals, and neutral carbon) ${ }^{[3-24]}$ and air molecules. The temperature of the plume dissipated as the plume expanded into the surrounding air, and there was nucleation and growth of self-assembled carbon nanoparticles in the plume. These nanoparticles later collided and aggregated into interwoven nanostructures to form a CNRN. The nanotopography and the altered nanochemistry of the fabricated nanonetwork is a function of substrate properties, ambient air vapor density, ionization energy, and complex dynamics of plume expansion. The ionization energy directly correlated with the laser parameters, such as the laser power, ultrashort pulse laser fluence, laser repetition, and beam scanning speed. 
The carbon nano-network dosage $\left(\rho_{n s}\right)$ is proportional to the number of ionized carbon nanoparticles $\left(N_{n p}\right)$ per pulse, which is directly proportional to the ultrashort pulse laser fluence, $f\left[J / \mathrm{cm}^{2}\right]=E_{p}[J] / A_{f}\left[\mathrm{~cm}^{2}\right]$, where $E_{p}$ is the laser pulse energy and $A_{f}$ is the effective laser beam area, which is inversely proportional to laser machining speed ${ }^{[3-25]}, \rho_{n s} \propto \frac{1}{S}(f)^{1 / 2}$. In this study, we used three different ultrashort pulse laser fluence of 4.43, 2.22 and $0.68 \mathrm{~J} / \mathrm{cm}^{2}$, and two different beam scanning speeds of 1 and $7.5 \mathrm{~mm} / \mathrm{s}$ to achieve variation in the nano-topography, chemistry and the yield of the created nano-network.

Both nanoribbon width and CNRN Index describe the physical morphology of the created CNRN platform. Table 3-1 showed the CNRN Index calculation. In calculating the CNRN Index, a dimensionless density number, representing the compactness of the arrangement of the CNRN clusters in the platform, was calculated using the nanovoid diameter of the created CNRN platform.

Table 3-1: Calculation of CNRN Index of the created CNRN platform for change in ionization energy from low to high

\begin{tabular}{|c|c|c|c|c|}
\hline $\begin{array}{c}\text { Ionization } \\
\text { Energy }\end{array}$ & $\begin{array}{c}\text { Nanovoid diameter } \\
(\mathbf{n m})\end{array}$ & $\begin{array}{c}\text { C-O bond } \\
\text { percentage }\end{array}$ & $\begin{array}{c}\text { Density } \\
\text { number }\end{array}$ & CNRN Index \\
\hline & $(\mathrm{a})$ & $(\mathrm{b})$ & $(\mathrm{c}=1000 / \mathrm{a})$ & $(\mathrm{d}=\mathrm{c} \mathrm{X} \mathrm{b})$ \\
\hline Low & 400 & 20 & 2.50 & 50 \\
\hline Medium & 250 & 22 & 4.00 & 88 \\
\hline High & 175 & 23 & 5.70 & 131 \\
\hline
\end{tabular}


A

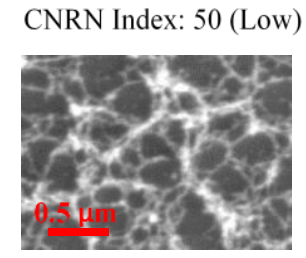

Density \# 2.5, C-O bond percentage 20

Low Energy

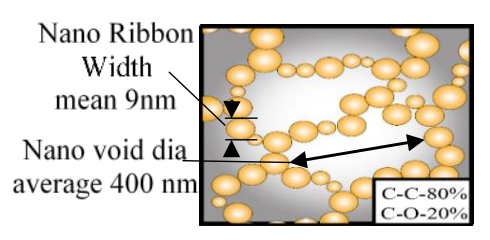

CNRN Index: 88 (Medium)

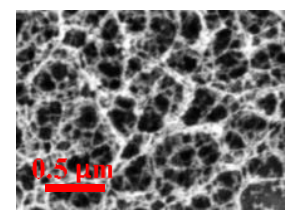

Density \# 4, C-O bond percentage 22
CNRN Index: 131 (High)

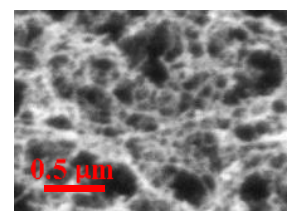

Density \# 6, C-O bond percentage 23 CNRN INDEX $\longrightarrow$ High Energy
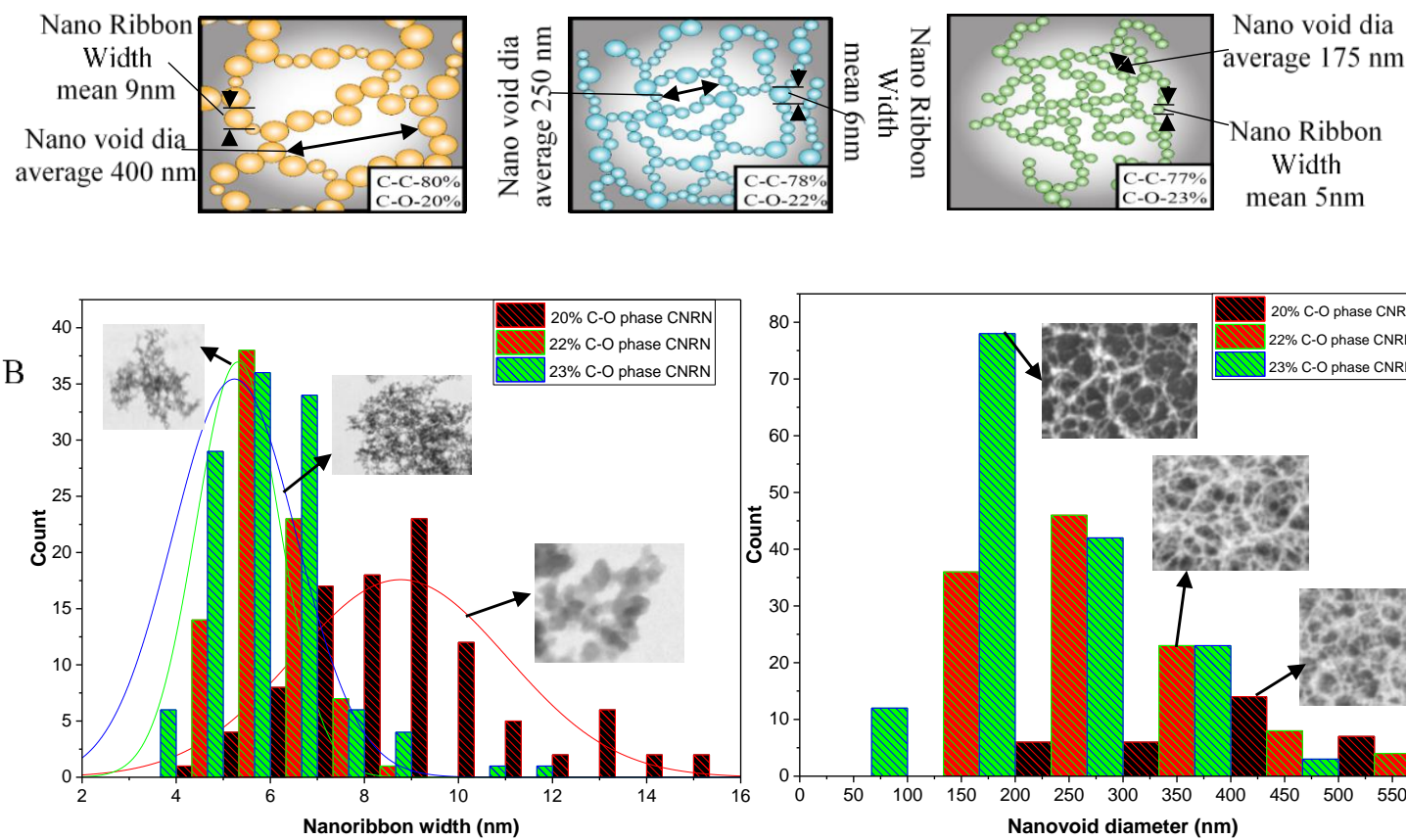

Nano void dia mean $5 \mathrm{~nm}$

Figure 3-3: (A) CNRN index of the synthesized $\mathrm{CNRN}$ platform with different $\mathrm{C}-\mathrm{O}$ percentage phases and density numbers at low, medium, and high ionization energies. (B) Nanoribbon width frequency histogram and nanovoid diameter frequency histogram of the created nanonetwork.

$\mathrm{CNRN}$ index is a combined representation of the $\mathrm{C}-\mathrm{O}$ bond percentage and the density number. The calculation of the CNRN index is based on the created CNRN platform that contains $\mathrm{C}-\mathrm{O}$ bonds of varying percentages. The untreated graphite contains only $\mathrm{C}-\mathrm{C}$ bond. Therefore, the CNRN index for untreated graphite as control might be assumed to be zero. CNRN index showed 
a positive increment with the increment of ionization energy from low to high. With the increase in the ionization energy, there is a gradual reduction in both the nanoribbon width and the nanovoid diameter, along with an increase in the yield, as evident in the SEM micrographs (Figure 3-3 A). Higher ionization energy tends to generate a denser plume and leads to a denser carbon nanostructure. The average size of the carbon nanoribbon width and the corresponding average nanovoid diameter varied from 9 to $5 \mathrm{~nm}$ and 400 to $175 \mathrm{~nm}$ with the increase in the ionization energy from 0.68 to $4.43 \mathrm{~J} / \mathrm{cm}^{2}$. The CNRN indices also change from 50 to 131 with the change in the ionization energy and therefore termed low, medium, and high CNRN index. The frequency histograms of the nanoribbon width distribution and the nanovoid diameter distribution of the CNRN platform (Figure 3-3B) show that higher ionization energy tends to have a narrower width distribution but has little effect on the void diameter distribution. This observation agrees with our previous observation of the femtosecond laser synthesis of the Si nanostructure. ${ }^{[3-26]}$

The characteristic XPS spectra of the graphite substrate and the created CNRN for different $\mathrm{C}-\mathrm{O}$ percentage phases on the graphite substrate along with a quantitative analysis of the major elements are presented in Figure 3-4 A-D. XPS spectra show two characteristic peaks at 285.08 and $533.08 \mathrm{eV}$ for the $\mathrm{CNRN}$ platform and the native graphite substrate, which can be ascribed to $\mathrm{C} 1 \mathrm{~s}$ and $\mathrm{O}$ 1s, respectively (Figure 3-4A). During the interaction of laser-mater in the presence of the surrounding atmosphere, there are neutral and reactive gases, such as nitrogen and oxygen. Hence, reactive oxygen takes part in the chemical transformation with the carbon ion and forms $\mathrm{C}-\mathrm{O}$ bonds. Therefore, the major chemical bonds present in the nanonetwork are $\mathrm{C}-\mathrm{C}$ and $\mathrm{C}-\mathrm{O}$ bonds. The percentage of oxygen in the CNRN platform increases with the increase in the ionization energy. Quantitative analysis of the major elements (Figure 3-4 B) shows that the percentage of $\mathrm{C}-\mathrm{O}$ bond is measured as 20,22 , and $23 \%$ at ionization energies of $0.68,2.22$, and 
$4.43 \mathrm{~J} / \mathrm{cm}^{2}$, respectively. Deconvolution of the XPS spectra was used to evaluate the $\mathrm{sp}^{2}$ and $\mathrm{sp}^{3}$ contents. Figure 3D shows the fittings of the deconvoluted XPS spectra of the C 1s peaks. The first peak position, which is at approximately $284.7 \mathrm{eV}$, corresponds to the $\mathrm{sp}^{2}$ carbon, ${ }^{[3-27]}$ whereas the peak centered near $285.5 \mathrm{eV}$ is interpreted as the $\mathrm{sp}^{3}$ hybridized carbon. ${ }^{[3-28]}$ Conversely, the fitting of the deconvoluted XPS spectra of the O 1s peaks (Figure 3-4 C) shows the presence of different oxygen-containing functional groups. The peak position at approximately $531.8 \mathrm{eV}$ corresponds to $\mathrm{C}=\mathrm{O}$ bonds, with the peaks centered near 532.88 and $533.32 \mathrm{eV}$ interpreted as the $\mathrm{C}-\mathrm{O}$ bonds that correspond to the chemisorbed oxygen. ${ }^{[3-29]}$

Figure 3-4 E shows the Raman spectra of the graphite substrate along with CNRNs synthesized at three levels of ionization energy. The native graphite shows a $\mathrm{G}$ band $\left(\sim 1575 \mathrm{~cm}^{-1}\right)^{[3-30]}$ and a D band $\left(\sim 1350 \mathrm{~cm}^{-1}\right)$, the two known bands of graphite. ${ }^{[3-31]}$ The presence of these two bands indicates that native graphite has a crystalline lattice structure containing a defect on the graphene surface. For the nanoribbon networks, the band peak positions shifted to the range of 1330 to 1365 $\mathrm{cm}^{-1}$ and 1555 to $1565 \mathrm{~cm}^{-1}$. The addition of $\mathrm{C}-\mathrm{O}$ bonds to the native graphite could be responsible for such peak shift. Two other prominent peaks also appeared at $2314 \mathrm{~cm}^{-1}$ and approximately $2700 \mathrm{~cm}^{-1}$. According to other researchers, ${ }^{[3-32,3-33]}$ these peaks are designated as $\mathrm{D}^{\prime}$ and $\mathrm{G}^{\prime}$, where $\mathrm{D}^{\prime}$ is a defect induced Raman band and G'-band appeared in the second order Raman spectra of crystalline graphite (without any kind of disorder) and corresponded to the overtone of the D-band. The $\mathrm{I}_{\mathrm{D}} / \mathrm{I}_{\mathrm{G}}$ ratio is the measure of the $\mathrm{sp}^{2}$ domain size in a graphitic structure including the $\mathrm{sp}^{3}$ and $\mathrm{sp}^{2}$ bonds ${ }^{[3-31]}$ and is also inversely proportional to the crystallite size. ${ }^{[3-34]}$ The $\mathrm{I}_{\mathrm{D}} / \mathrm{I}_{\mathrm{G}}$ ratio of graphite is 0.60 . The $\mathrm{I}_{\mathrm{D}} / \mathrm{I}_{\mathrm{G}}$ ratio showed an increasing trend ranging from 0.74 to 0.81 when the $\mathrm{C}-\mathrm{O}$ phases changes from 20 to $23 \%$ (Figure 3-4D). This shows that the nanonetwork created at a higher ionization energy has a smaller crystallite size. 
$\Lambda$

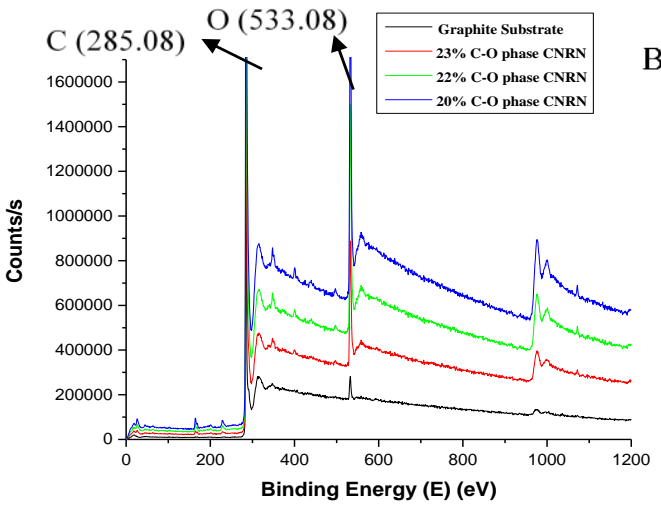

$\mathrm{C}$

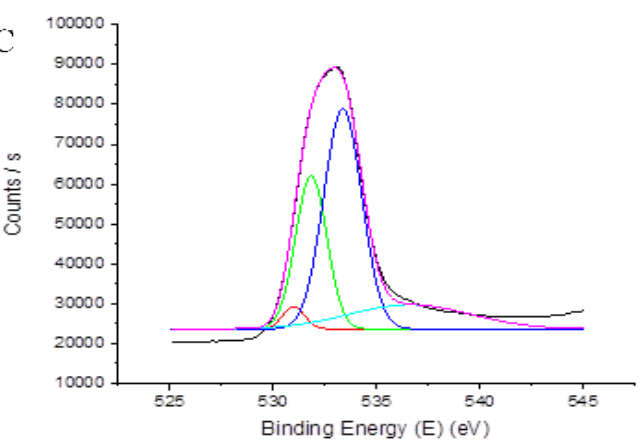

Deconvoluted O1s XPS spectra of CNRN
B

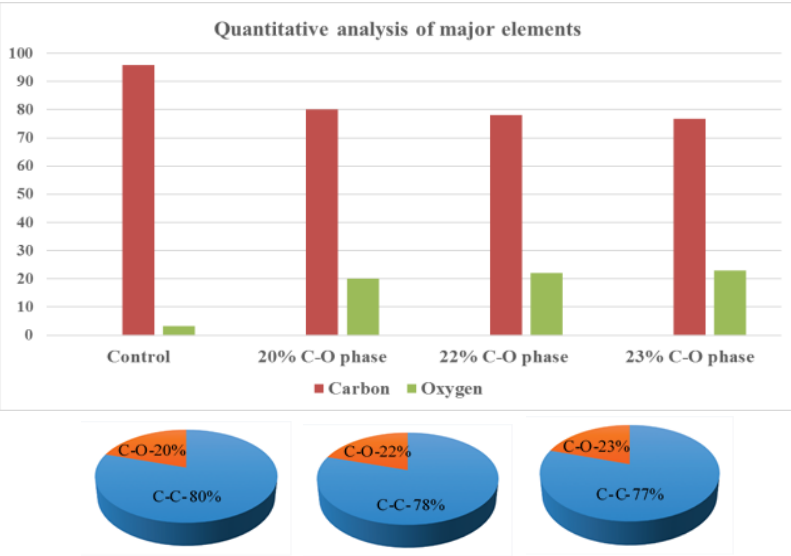

D

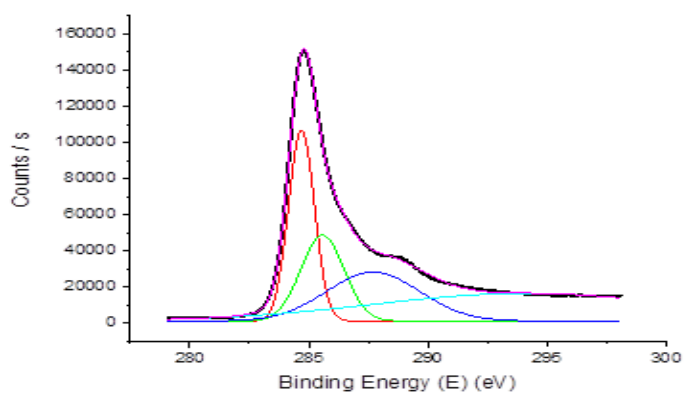

Deconvoluted C1s XPS spectra of CNRN
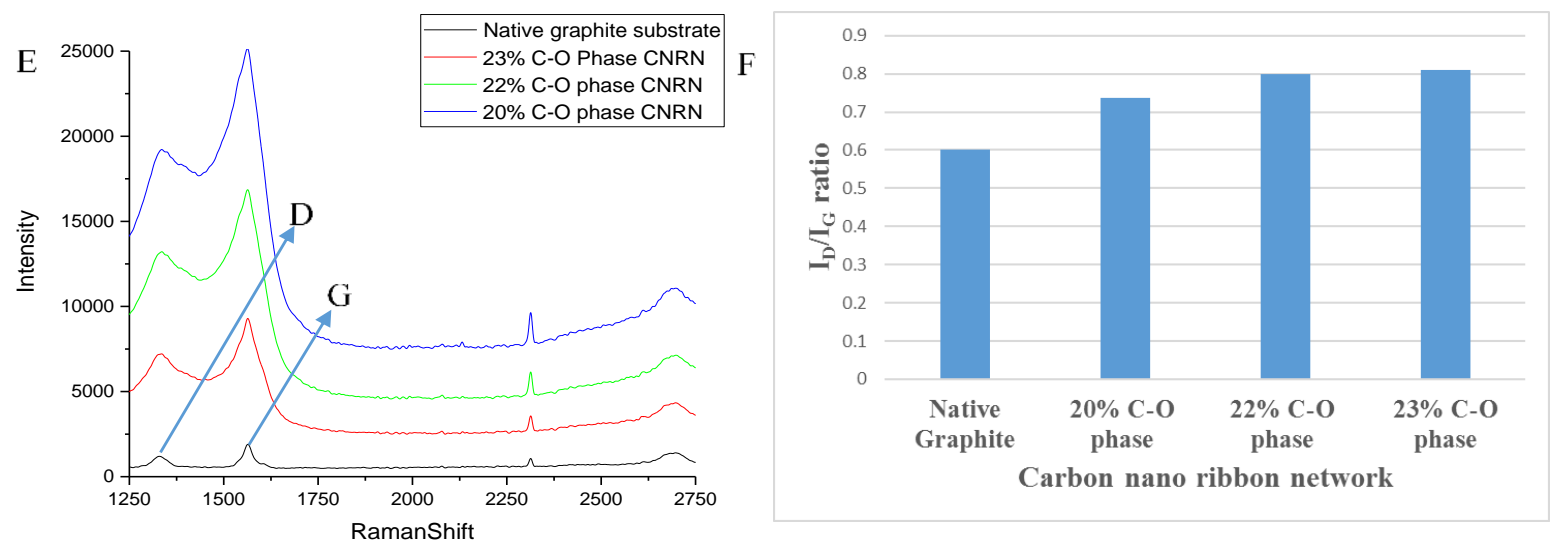

Figure 3-4: (A) XPS spectra of the carbon nanoribbon network having different C-O percentage phases with the (B) corresponding quantitative analysis of the major elements in the created carbon nanoribbon network; (C) deconvoluted O1s XPS spectra and (D) deconvoluted C1s XPS spectra for CNRN platform; (E) Micro-Raman spectra of the graphite substrate and the graphite substrate with the carbon nanoribbon network having different $\mathrm{C}-\mathrm{O}$ percentage phases and the $(\mathrm{F})$ influence of different $\mathrm{C}-\mathrm{O}$ percentage phases on the $\mathrm{I}_{\mathrm{D}} / \mathrm{I}_{\mathrm{G}}$ ratio. 


\subsubsection{Fibroblasts and HeLa cell response to the bio active carbon nanoribbon network}

Fibroblasts and HeLa cells were cultured for 24 and $48 \mathrm{~h}$ on both the graphite substrate (experimental control) and the CNRN platforms to study the attraction, adhesion, and proliferation behavior of these cell lines. To analyze the experimental outcomes, the results of the control have been taken as the reference. The efficiency of the CNRN platform to attract cells, their viability, and morphology were examined in terms of the nanonetwork density and the $\mathrm{C}-\mathrm{O}$ bond concentration.

\subsubsection{Time dependent cell fate}

Figure 3-5 demonstrates the overall cellular responses of fibroblasts and HeLa cells after 24 and $48 \mathrm{~h}$. A distinct difference was observed in terms of cell attraction on CNRN platforms in comparison to the graphite substrate. For graphite substrate, no or few cells were attached, fibroblasts or HeLa cells. On the other hand, affirmative attraction, adhesion, and growth were observed on the CNRN platform after $24 \mathrm{~h}$. The fibroblast showed overextended filopodia and actin filament, whereas HeLa exhibited only a minor filopodia extension.

The fate of the cells takes different directions after 48 hours on CNRN. Fibroblasts showed tissuelike structure because of growth enhancement. On the contrary, elongated HeLa cells stopped growing and seemingly decreased in size, formed round shape cocoon-like structure. 


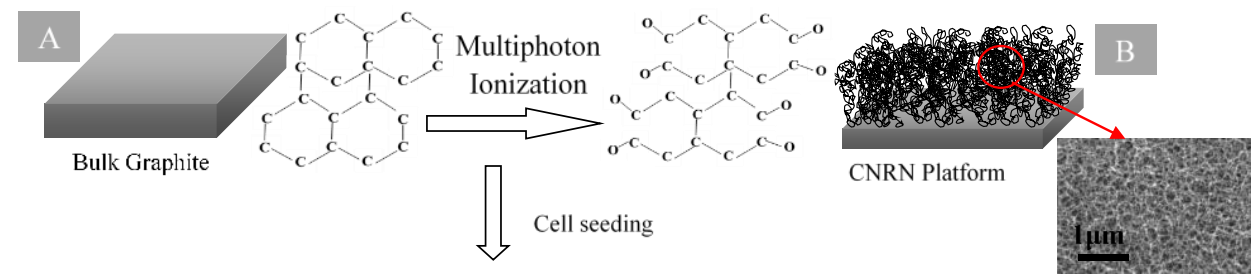

No response

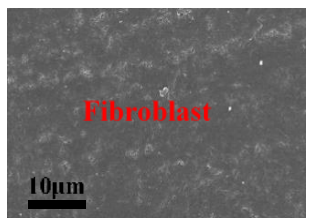

Affirmative adhesion and growth $(24 \mathrm{~h})$
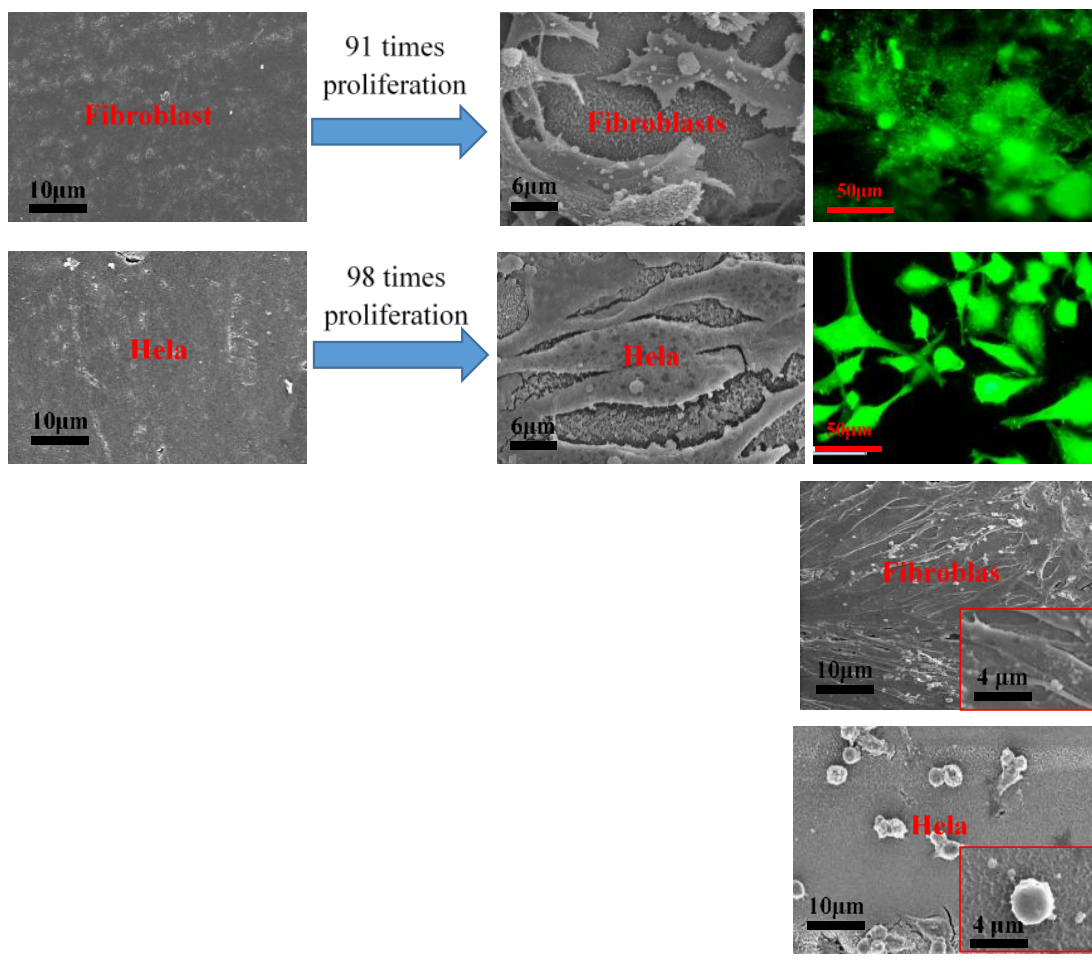

Figure 3-5: Fibroblasts and HeLa cell responses on (A) native graphite substrate and the (B) bio active carbon nanoribbon network platform. The graphite substrate has no cell response for both fibroblasts and HeLa cell after $24 \mathrm{~h}$. The created CNRN platform has positive cell response with a cell selective cytophilic/cytotoxic response on fibroblasts and HeLa cells after $24 \mathrm{~h}$ and $48 \mathrm{~h}$, respectively.

\subsubsection{Effect of varying dosage of the CNRN and C-O concentration}

Subsequently, the adhesion behavior of fibroblasts and HeLa cells with a varying dosage of CNRN and changing $\mathrm{C}-\mathrm{O}$ concentration of bioactive $\mathrm{CNRN}$ on the substrate surface was observed. The number of cells that adhered to the $\mathrm{CNRN}$ platforms with varying $\mathrm{C}-\mathrm{O}$ concentrations and differing CNRN dosages were counted. The thickness of the deposited fibrous nanostructure layer for different laser beam scanning speeds (for this experiment 1 and $7.5 \mathrm{~mm} / \mathrm{s}$ ) has been designated 
as the dosage of CNRN used for cell experiments. The layer thickness varied from a maximum of $0.060 \mu \mathrm{m}$ to a minimum of $0.002 \mu \mathrm{m}$ for the previously mentioned two scanning speeds. This result is in agreement with our previous observation of femtosecond laser synthesis of $\mathrm{Si}$ nanostructure. ${ }^{[3-35]}$ High CNRN dosage corresponds to maximum thickness of the deposited fibrous nanostructure, whereas low CNRN dosage has minimum thickness of the deposited fibrous nanostructure layer. As observed from the graph presented in Figure 3-6, the effect of a change in the CNRN dosage was more dominant in the fibroblasts compared with the HeLa cells after $24 \mathrm{~h}$ of culture. With the change in the CNRN dosage from low to high, the number of fibroblast cells adhered to the CNRN platform almost doubled in the case of the high CNRN dosage. However, the same change in the CNRN dosage did not significantly affect HeLa cell adhesion. There was a distinct positive increase in fibroblast cell adhesion with the $\mathrm{C}-\mathrm{O}$ concentration increment for high CNRN dosage platform. Because of the formation of a tissuelike structure in the case of fibroblast cells after $48 \mathrm{~h}$ of culture, we were merely differentiating individual fibroblast cells on the $\mathrm{CNRN}$ platform with different $\mathrm{C}-\mathrm{O}$ percentage phases with varying dosage, which restricted the counting of the fibroblast cells. By contrast, after $48 \mathrm{~h}$ of culture, there was a great detachment of the HeLa cells (almost half) compared with the earlier adhesion after $24 \mathrm{~h}$ of culture. There was a decreasing trend of the adhered $\mathrm{HeLa}$ cells in terms of $\mathrm{C}-\mathrm{O}$ concentrations irrespective of $\mathrm{CNRN}$ dosage.

\subsubsection{Cell Morphology.}

An in-depth analysis of the effect of CNRN characteristics, namely, nanoribbon width and CNRN index, on the cell morphology was carried out using SEM and FM micrographs. Both fibroblasts and HeLa showed highest affinity of cellular attraction with the CNRN platform of high CNRN 


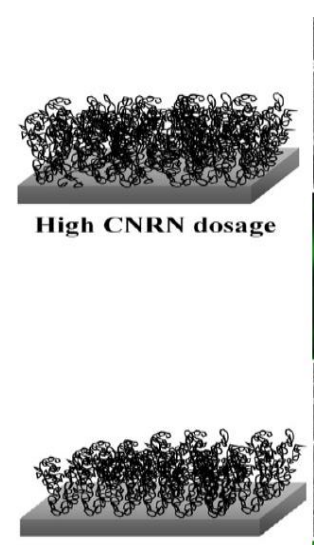

Low CNRN dosage
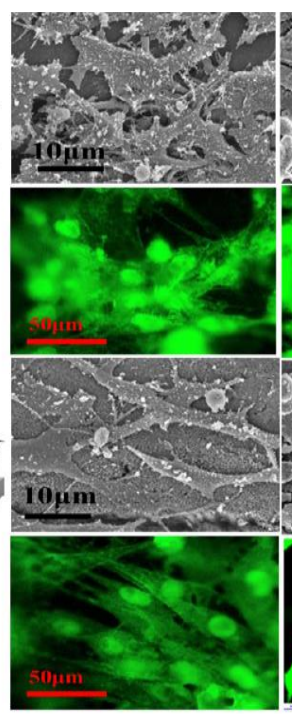

Fibroblast

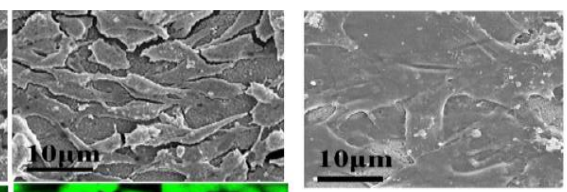

$10 \mu \mathrm{m}$
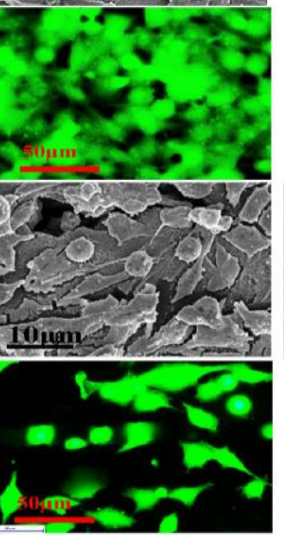

HeLa

24 hours

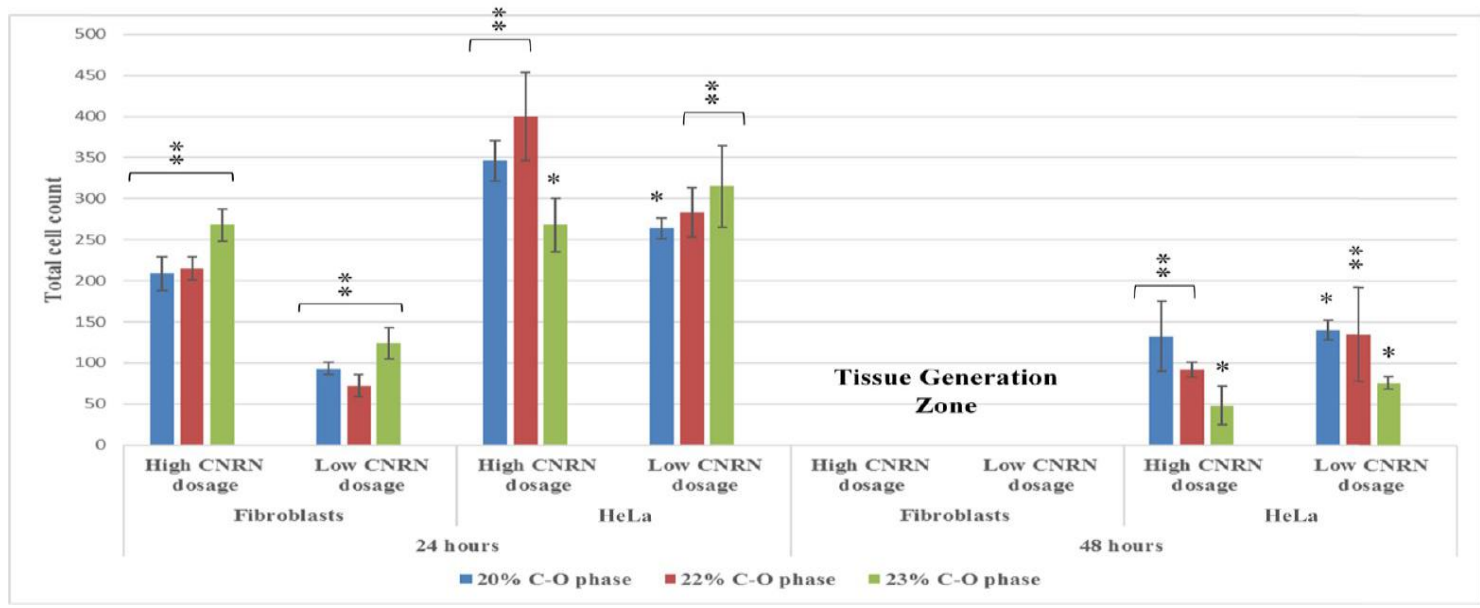

Figure 3-6: fibroblast and HeLa cell adhesion and proliferation after $24 \mathrm{~h}$ and $48 \mathrm{~h}$, on varying dosage of the CNRN and C-O concentration; Experiments were repeated with $n=3$. Statistical significance is shown with $* \mathrm{p}<0.05, * * \mathrm{p}<0.01$.

index with smaller nanoribbon width. There was a gradual increment in the cellular attraction of both the cells with CNRN platforms having gradual increment of CNRN indices. Well-adhered, stretched, and an extended cellular morphology of both cell lines was observed after $24 \mathrm{~h}$ of culture. The fibroblasts were well-spread and flattened, and they overlapped each other, covering the nanonetwork platform; their filopodia and actin filaments overextended along the nanonetwork 
zones. The HeLa cells were also well-spread and elongated, covering the nanonetwork with short filopodial extensions along the nanonetwork zones. It is remarkable to mention that an analogous tendency of cell adhesion on all types of CNRN platforms, regardless of their crystallinity, was observed in both the cell lines. The findings from the fluorescence study [Figure 3-7 A (v, vi), $\mathrm{B}(\mathrm{v}, \mathrm{vi}), \mathrm{C}(\mathrm{v}, \mathrm{vi})]$ were in agreement with the SEM micrographs for both fibroblasts and HeLa cells. The analysis of the SEM micrographs of the cells cultured for $48 \mathrm{~h}$ [Figure 3-7A (ii, iv), B(ii,iv),C(ii,iv)] showed a contrary result for the HeLa cells. The fibroblasts that adhered to the CNRN platform were more intimately organized. The overlapped fibroblast cells that interlinked with each other showed a tissue like morphology. The prominence of this trend was more visible on the CNRN platform with a high CNRN index. Conversely, the HeLa cells stopped their proliferative behavior and contracted to form a round, cocoon like morphology. The same trend was observed for all three different CNRN indices. For both fibroblasts and HeLa cells, the wellspread elongated shape with extended filopodia is suggestive of healthy cells that have increased cellular interaction with the CNRN network, whereas, round-shaped HeLa is indicative of nonproliferating/apoptotis-like cells.

Furthermore, a detailed comparison of the morphological change of the individual fibroblasts and HeLa cells that adhered and expanded on the CNRN platform of different $\mathrm{CNRN}$ indices and $\mathrm{C}-\mathrm{O}$ bond percentages after 24 and $48 \mathrm{~h}$ of growth was carried out, as depicted in Figure 3-8. 


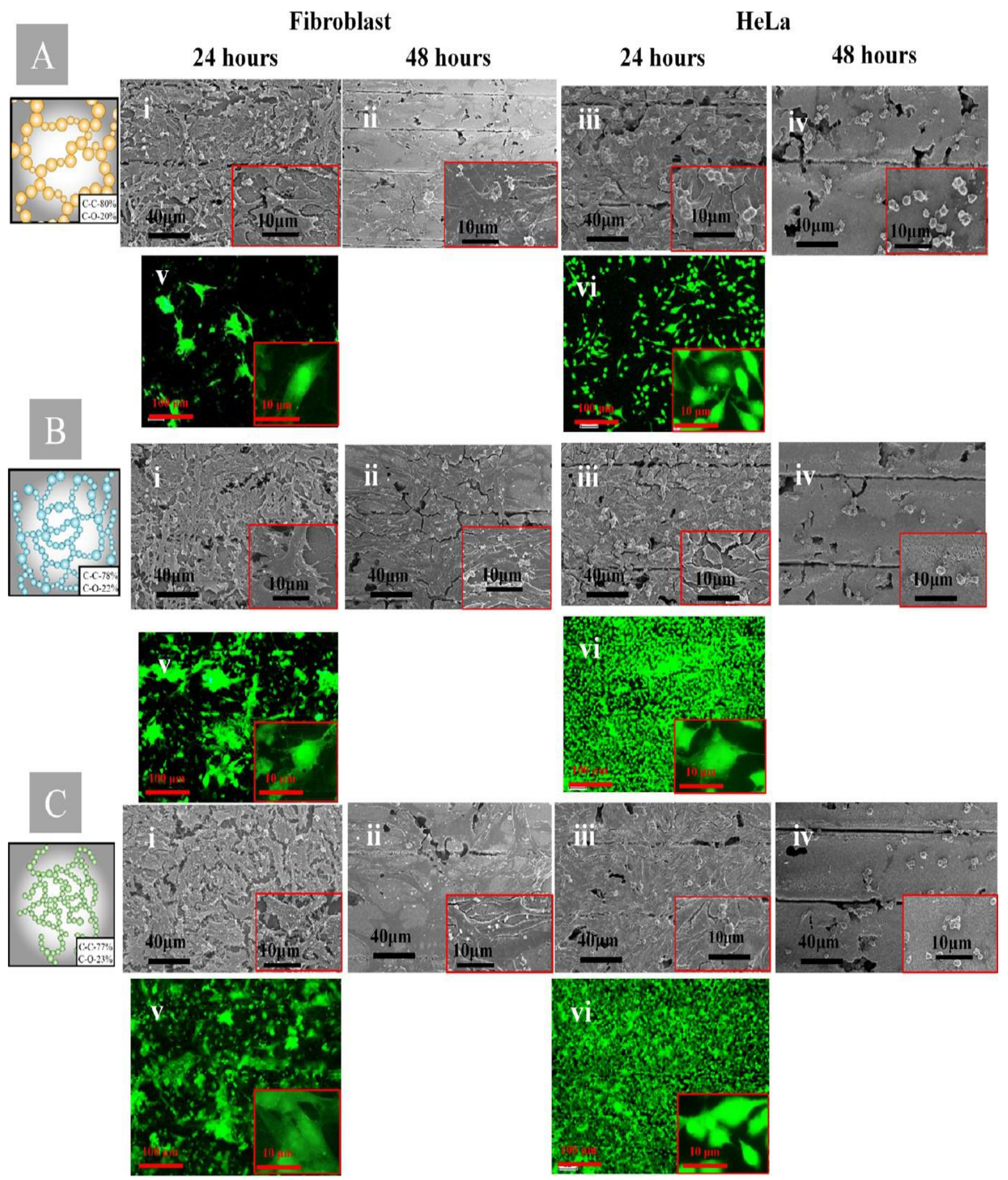

Figure 3-7. SEM micrographs of the fibroblasts and HeLa cells adhered to the (A) $20 \% \mathrm{C}-\mathrm{O}$ phase, (B) $22 \% \mathrm{C}-\mathrm{O}$ phase, and (C) $23 \% \mathrm{C}-\mathrm{O}$ phase, along with the corresponding $\mathrm{FM}$ images after $24 \mathrm{~h}$ of culture. 


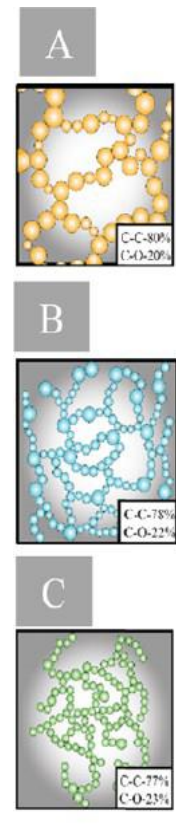

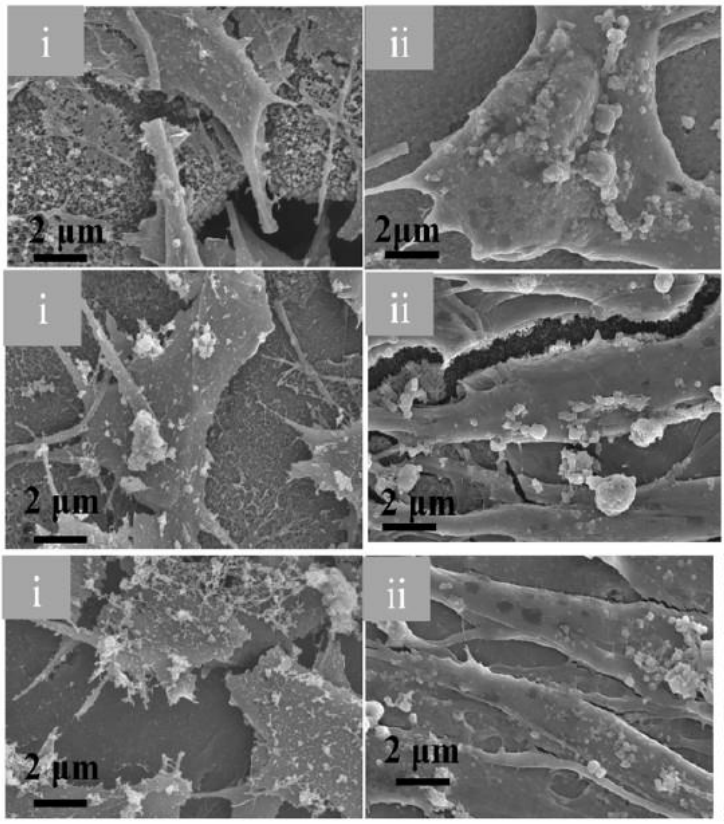

24 hours
48 hours

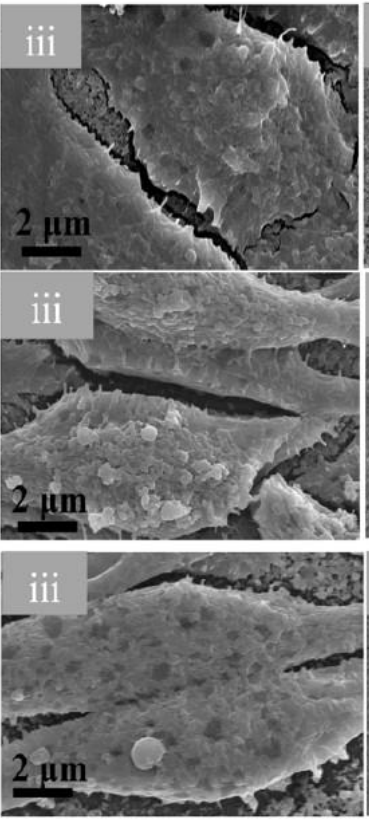

24 hours
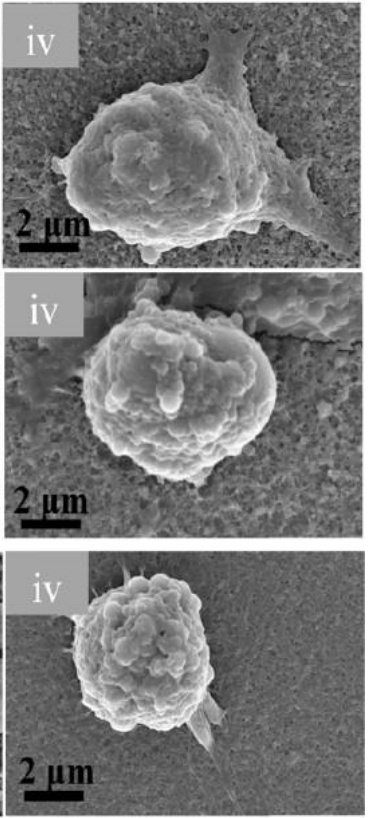

48 hours

Fibroblast

HeLa

Figure 3-8. Comparison of the morphology of individual fibroblasts and HeLa cells adhered on the various $\mathrm{C}-\mathrm{O}$ percentage phase $\mathrm{CNRN}$ platforms; SEM micrograph of (A) fibroblasts [A(i, ii)] and HeLa cells [A(iii, iv)] on the $20 \% \mathrm{C}-\mathrm{O}$ phase, (B) fibroblasts [B(i, ii)] and HeLa cells [B(iii, iv)] on the $22 \% \mathrm{C}-\mathrm{O}$ phase, and (C) fibroblasts [C(i,ii)] and HeLa cells [C(iii,iv)] on the $23 \% \mathrm{C}-\mathrm{O}$ phase after 24 and $48 \mathrm{~h}$ of culture, respectively.

The morphology of the fibroblast seeded on a low CNRN index having $20 \% \mathrm{C}-\mathrm{O}$ bond percentage phase [Figure 3-8A(i)] showed random orientation and a well-defined flattened cellular organism with short filopodia extension. With increase in the CNRN index, the flattened cellular organisms were stretched, and there were numerous overextended filopodia and focal adhesion points, that is, the cell-nanostructure interaction point increased (Figure 3-8 B(i), C(i)). On the other hand, HeLa showed organized orientation with almost the same type of flattened cellular organisms and short filopodia on all three different CNRN platforms, regardless of the change in the CNRN index [Figure 3-8A(iii), B(iii),C(iii)]. With time, the fibroblasts were organized, interlinked with each 
other, and formed a tissue-like morphology [Figure 3-8A(ii), B(ii), C(ii)]. Conversely, after $48 \mathrm{~h}$ of growth, the flat, elongated HeLa cells seemingly decreased in size and formed a round-shaped cocoon, although their short length filopodial extension strongly adhered to the surface of the nanonetwork [Figure 3-8A(iv), B(iv), C(iv)].

Finally, FM was performed to investigate the cell phenotype and nucleus of the adhered fibroblasts and HeLa cells on the CNRN platform having different CNRN indices of varying $\mathrm{C}-\mathrm{O}$ bond percentage phases. Figure 3-9 presents the FM images of the actin cytoskeleton and nucleus of the HeLa and fibroblast cells stained in green and blue, respectively. The results obtained from FM were consistent with the SEM observations of the cell morphology and the adhesion mechanism. Contractile stress fibers, which play an important role in cell adhesion and migration, ${ }^{[3-36]}$ were observed for cells on all three platforms [Figure 3-9 A (ii, v), B(ii,v),C(ii,v)]. The nucleus of both type of cells adhered to the three different $\mathrm{C}-\mathrm{O}$ percentage phases of the CNRN showed a consistent trend and indicated the same morphological characteristic of those cells.

\subsubsection{Characteristics of Growing Cells.}

Additionally, a comparative quantitative analysis of the dissimilar shape of the fibroblasts and HeLa cells on the CNRN platforms (Figure 3-10) was performed. The cell shape could be treated as a good indicator of the cell response to the nanonetwork, for example, a well-spread polygonal cell shape with extended filopodia was indicative of healthy cells and had increased cellular interaction with the CNRN platforms, and a nonflattened round cell shape revealed

nonproliferating cells. ${ }^{[3-37,3-38]}$ The number of attached elongated cells and round cells were separately marked for both fibroblasts and the HeLa cells to understand the proliferation of healthy cells on the CNRN platforms. As observed from the graph in Figure 3-10, the number of attached elongated fibroblast 


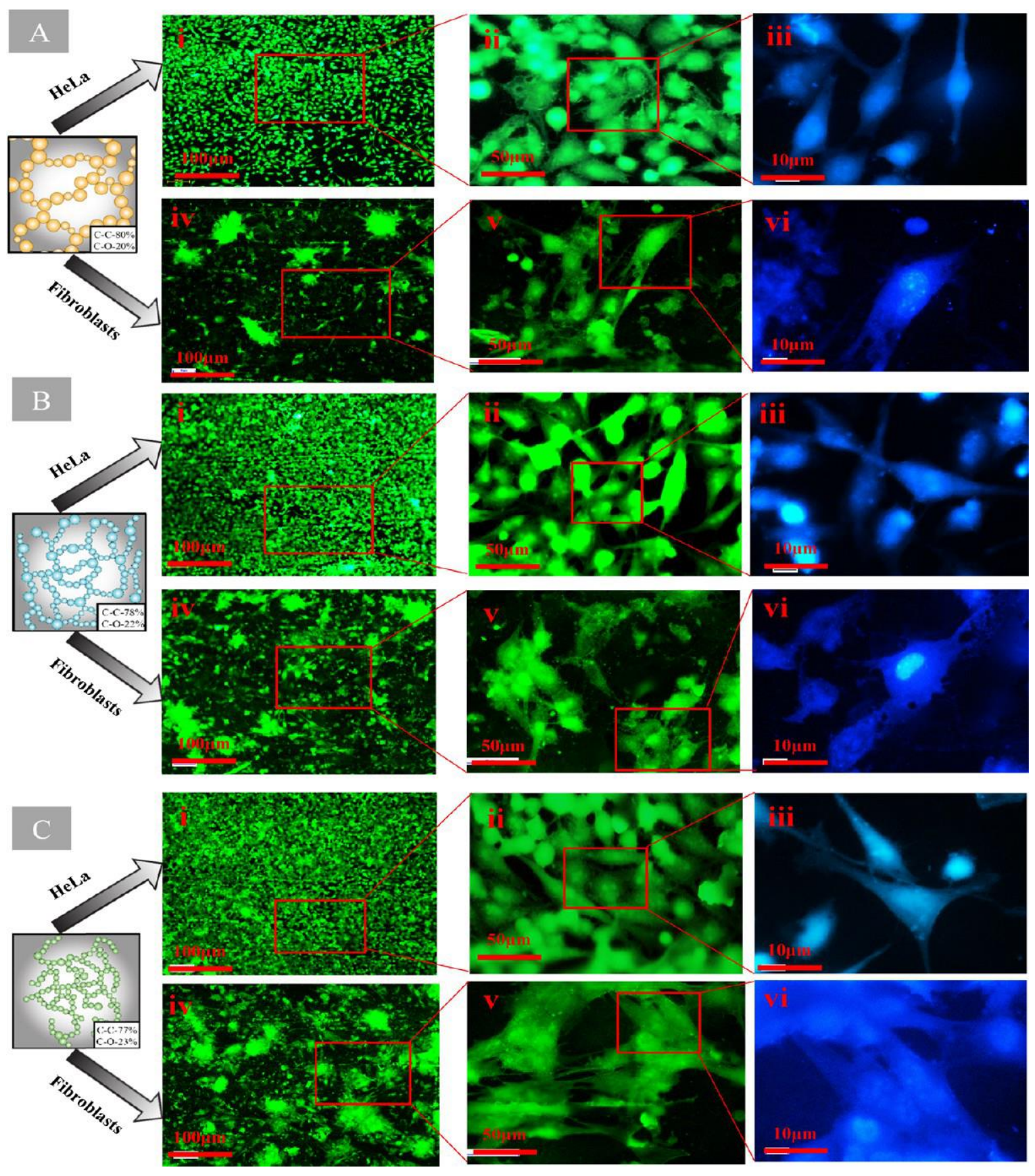

Figure 3-9. FM images of the HeLa cell and fibroblast cytoskeletons stained in green and the nucleus stained in blue attached to the $\mathrm{CNRN}$ with (A) $20 \% \mathrm{C}-\mathrm{O}$ dominant phase, (B) $22 \% \mathrm{C}-\mathrm{O}$ dominant phase, and (C) $23 \% \mathrm{C}-\mathrm{O}$ dominant phase. 
cells had a positive increase with the change in the CNRN index from low to high. However, the number of round cells had an almost steady trend over the said platforms. After $48 \mathrm{~h}$ of culture, the individual counting of fibroblasts was not possible because of the formation of the tissuelike structure. On the other hand, for CNRN platforms with a low CNRN index, more elongated HeLa cells were attached in comparison to that with a high CNRN index. Rounded HeLa cells had an exactly opposite trend. After 48 h of culture, greater than $90 \%$ of the attached HeLa cells were round compared with the elongated cells.

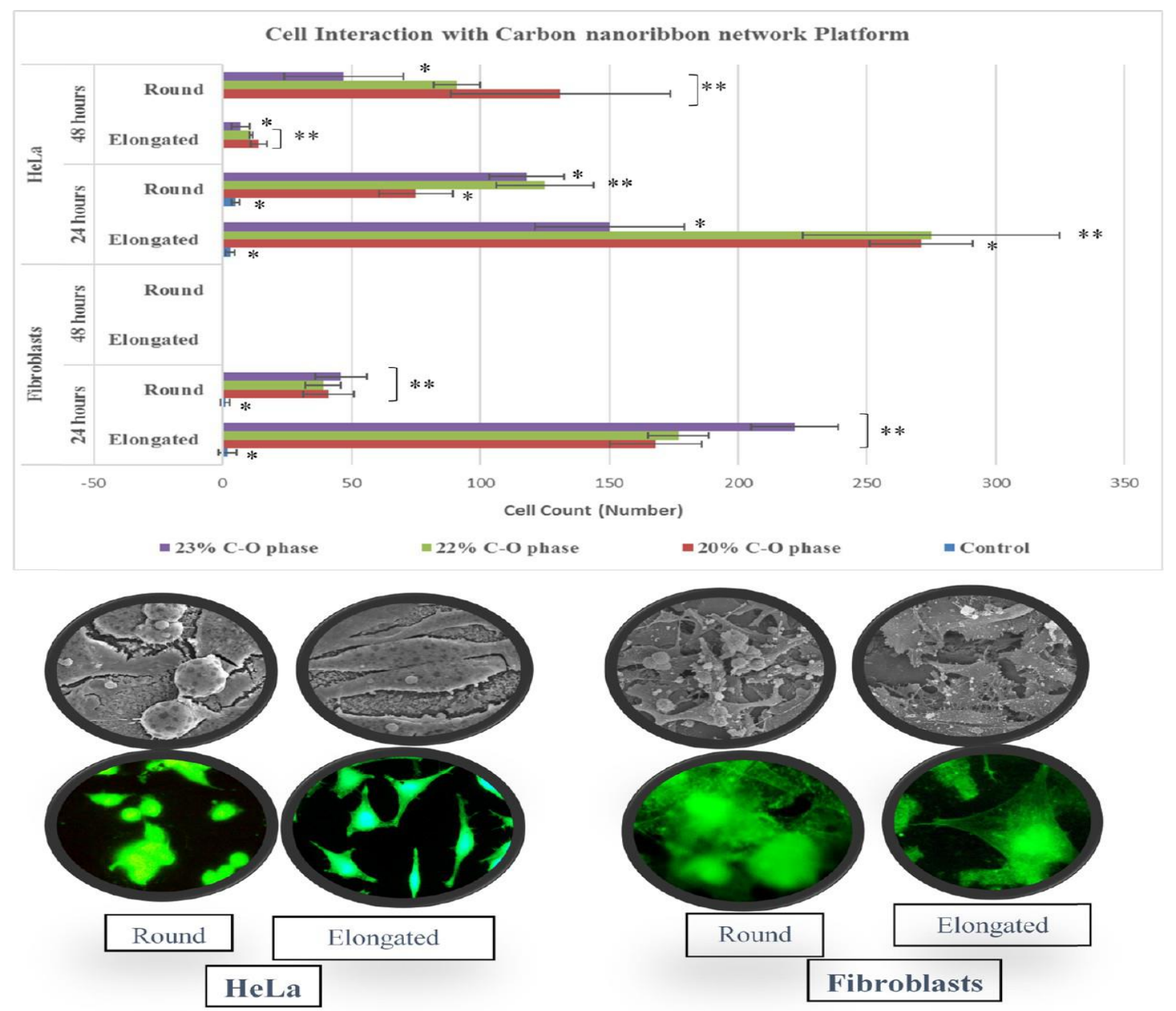

Figure 3-10. Quantitative analysis of the fibroblasts and HeLa cells on the CNRN with different percentage $\mathrm{C}-\mathrm{C}$ dominant phases. The error bars show the standard error of the mean; experiments were repeated with $\mathrm{n}=3$. Statistical significance is shown with $* \mathrm{p}<0.05$ and $* * \mathrm{p}<0.01$. 


\subsubsection{CNRN Uptake.}

Cellular uptake might be a mechanism that is involved in the cytophilic/cytotoxic behavior of the fibroblasts and HeLa cells in the presence of the CNRN platform over time. At the time of cell adhesion, the cells preferentially attach to the areas of higher protein adsorption, that is, CNRN platforms. ${ }^{[3-39,3-40,3-41]}$ After initial attachment, it is hypothesized that the contiguity of cells to the CNRN platform causes the uptake of constructive components of the CNRN platform, namely, CNRN clusters. The cell type and the nanoparticle size, shape, and surface charge determine the endocytic process through which nanoparticles are taken up by the cells once they are adsorbed onto the cell surface, ${ }^{[3-42,3-43]}$ and endocytosis is the major process in this regard. ${ }^{[3-44,3-45]}$ Figure 3-11 explains the effect of CNRN clusters uptake caused by endocytosis on both cell lines.
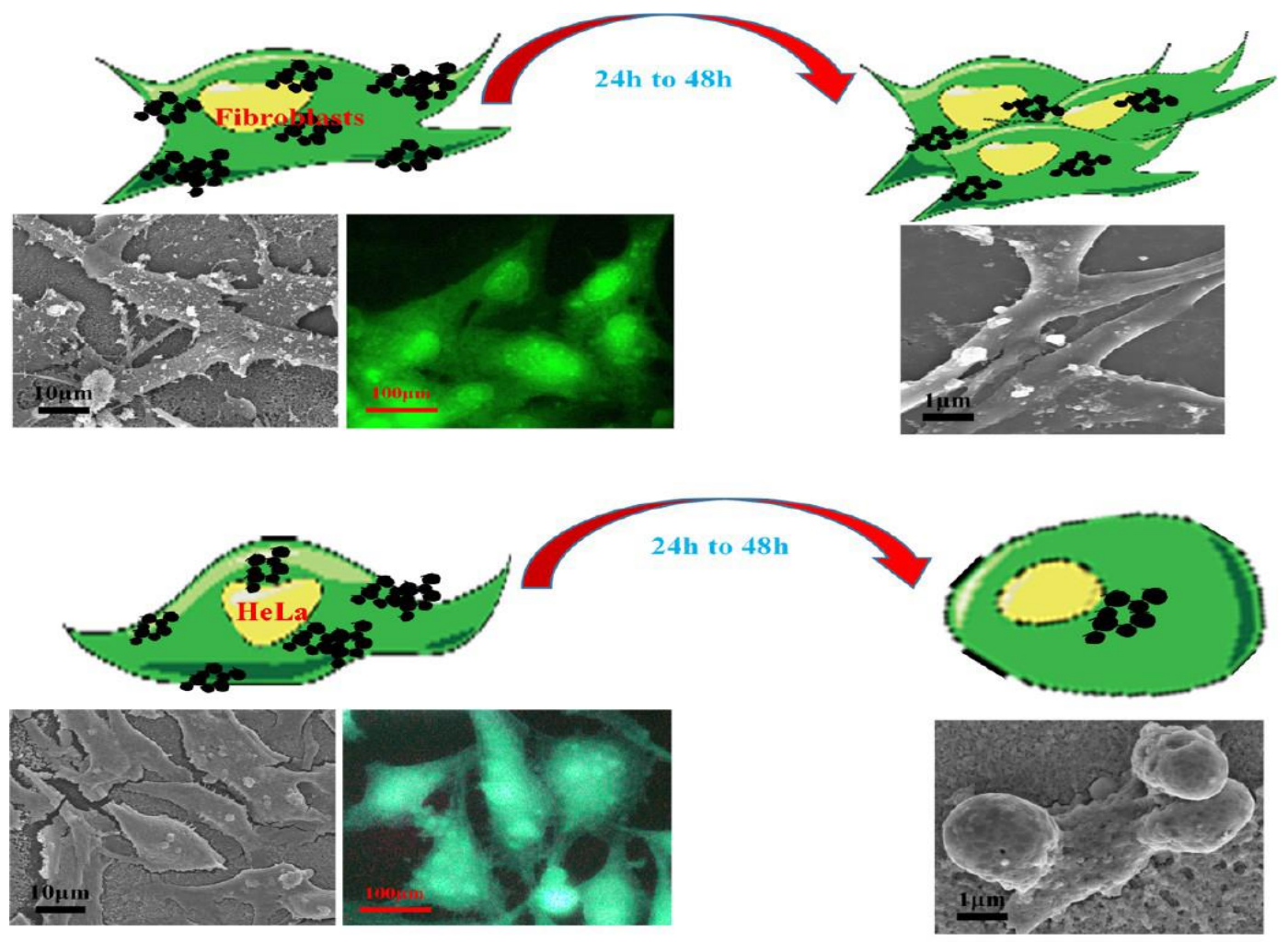

CNRN Cluster Endocytosis

Figure 3-11. Effect of CNRN cluster endocytosis of the HeLa cell and fibroblast cytoskeletons attached to the CNRN platform. 
Both SEM and FM micrographs evidenced internalized CNRN clusters because of endocytosis in the fibroblasts and HeLa cells, and consecutive results after $48 \mathrm{~h}$ showed the contrary cytophilic/cytotoxic effect of the CNRN clusters on fibroblasts and HeLa cells. Fibroblasts showed transformation to a tissue like structure, whereas HeLa resulted in rounded apoptotis-like cells. Detection of the trace of existence of the CNRN clusters in the cell cytoskeleton that was activated upon the uptake of CNRN clusters into the cell membrane was identified using EDX. The elemental mapping (Figure 3-11) might further elucidate the cytophilic/cytotoxic behavior of the CNRN platform over time on fibroblasts and HeLa cells.

It is evident from the elemental mapping that both the fibroblasts and HeLa cells have an increasing trend in adhesion and proliferation with respect to the spreading areas for increasing CNRN indices with varying $\mathrm{C}-\mathrm{O}$ bond percentages in a $24 \mathrm{~h}$ time frame. However, over time, the $\mathrm{CNRN}$ clusters induced apoptotis-like behavior on HeLa, leaving no effect on fibroblasts. From the EDX analysis, it is evident that there was internalization of CNRN clusters in both the fibroblasts and HeLa cells. The spreading pattern of oxygen mapping on fibroblasts, as shown in Figure 3-12 (A5, C5, and E5), indicated no hindering effect of oxygen, which was because of the presence of $\mathrm{C}-\mathrm{O}$ bond in the CNRN cluster, on fibroblast cells in tissue generation over time. On the other hand, the abundance of single area specific accumulation of oxygen in Figure 3-12 (B5, D5, and F5) might explain the apoptotis-like function of the CNRN clusters on HeLa cells. It is hypothesized that the contiguity of the cells to the CNRN platform causes the uptake of CNRN clusters. This interaction leads to a loss of cellular integrity, and there is a potential for growth impediment. ${ }^{[3-46-3-49]}$ According to previous studies, cell adhesion to nanostructures varies depending on the cell line, and for instance, fibroblasts are less sensitive to the surface properties compared with HeLa cells and are known to adhere and grow well on those surfaces. ${ }^{[3-39]}$ At the same time, fibroblasts show 
greater cytoskeleton stiffness compared with HeLa cells. ${ }^{[3-50,3-51]}$ It might be hypothesized that the $\mathrm{C}-\mathrm{O}$ bond structure over time induced some cytotoxic effect on the HeLa cells. Although surviving the early stage of adhesion, HeLa cells, because of the internalized CNRN clusters containing the $\mathrm{C}-\mathrm{O}$ bond might result in cell shrinkage and apoptosis-like functions over time.
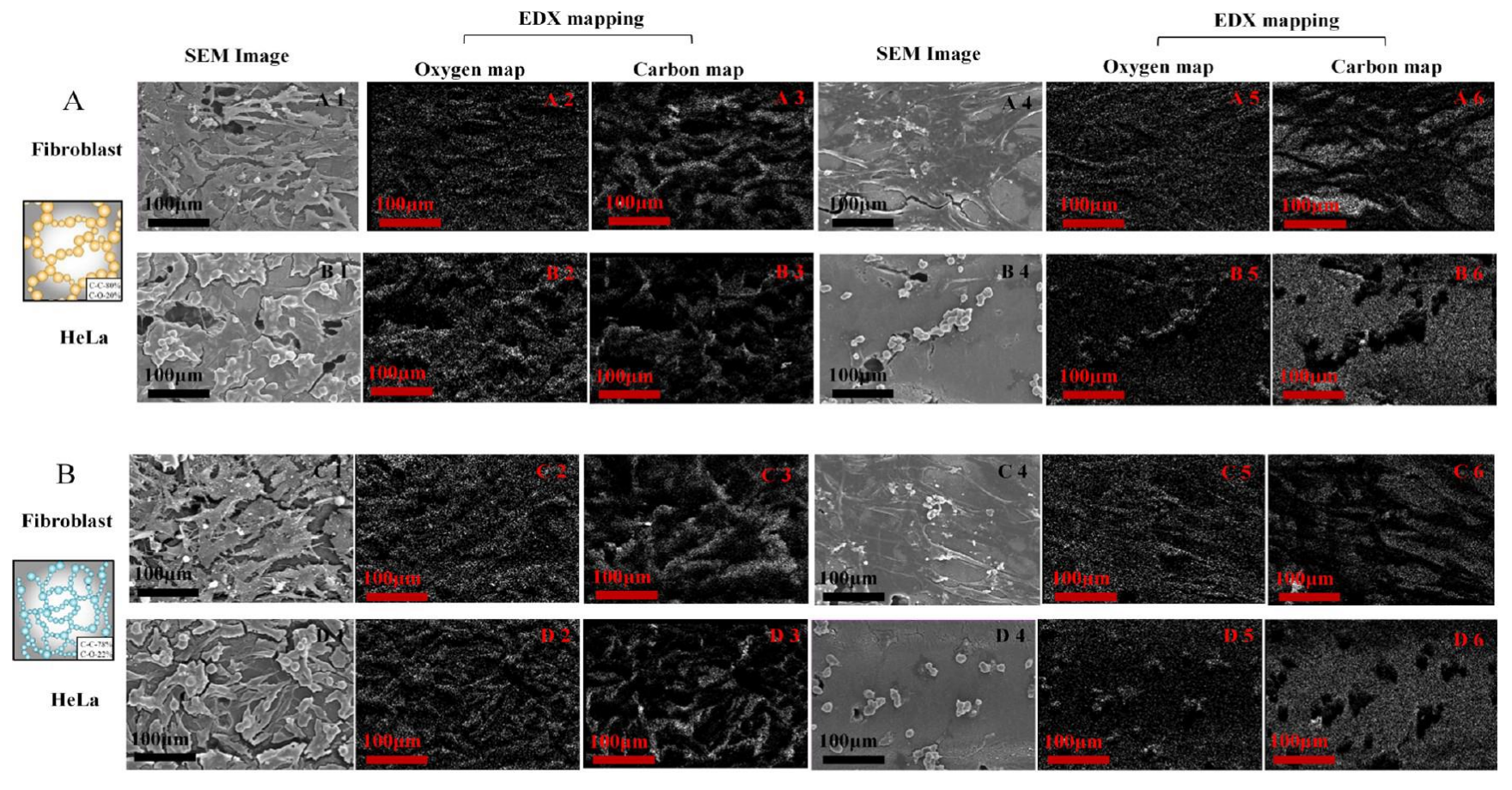

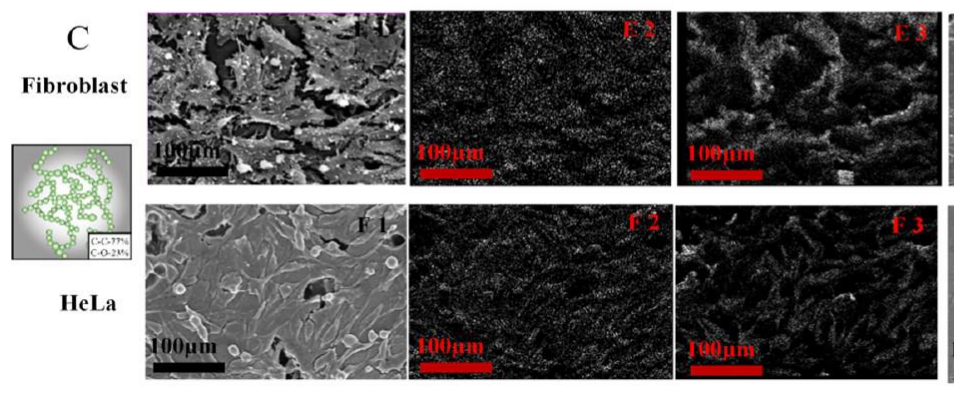

24 Hour

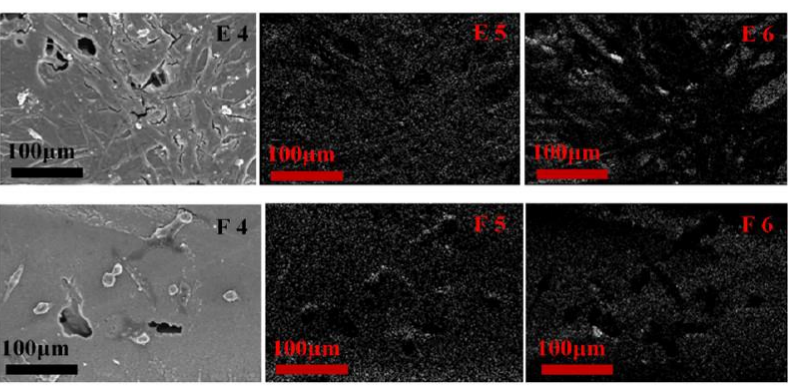

48 Hour

Figure 3-12. EDX mapping images of the HeLa and fibroblast cytoskeletons attached to the CNRN with (A) 20\% C-O, (B) 22\% C-O, and (C) 23\% C-O dominant phases. 


\subsection{Discussions}

\subsubsection{CNRN Formation.}

In biomaterial-cell interactions, the cell response to the material is significantly governed by the physicochemical properties of the surface. ${ }^{[3-52]}$ The incorporation of suitable topographical and chemical properties in the nanonetwork containing the substrate is important in biomaterial research to regulate the required cellular function for a biomedical application. In this study, the bioactivation of the graphite surface was achieved with the aid of ultrashort femtosecond laser processing in an ambient atmosphere. Mechanical fragmentation, homogeneous nucleation, and vaporization were the primary steps that led to the ablation of the graphite surface, which was irradiated using femtosecond laser pulses. ${ }^{[3-53]}$ A reactive high-temperature plasma plume created a combination of neutral carbon $(\mathrm{C})$, carbon radicals $(\mathrm{C} 2, \mathrm{C} 3)$, and carbon ions $(\mathrm{C}+, \mathrm{C} 2+)^{[3-54]}$ The heavier radicals and the neutral carbon reside at the bottom of the expanding plasma plume, whereas the carbon ions localize at the top of the plume. The change in the ionization energy from low to high controls the component mixture of the plume and has a significant impact on the topography variation and nanochemistry of the created nanoribbon network. The carbon nanospecies present in the plasma plume, while condensed and aggregated, formed an interwoven nanonetwork. With an increase in the ionization energy from low to high, the CNRN index of the created weblike interlinked CNRN changed from low to high. The ambient atmospheric gas containing oxygen atoms encountered the boundary of the expanding plume and had a chemical interaction with the carbon ions, resulting in the formation of $\mathrm{C}-\mathrm{O}$ molecular bonds that were deposited along with the $\mathrm{C}-\mathrm{C}$ molecules when condensation took place. The nanochemistry of the created $\mathrm{CNRN}$ platform comprised two major molecules, $\mathrm{C}-\mathrm{C}$ and $\mathrm{C}-\mathrm{O}$, and the mixture of this combination varies with the change in the ionization energy. The percentage of the $\mathrm{C}-\mathrm{O}$ and or 
$\mathrm{C}=\mathrm{O}$ molecules increased with an increase in the ionization energy. The FE-SEM micrograph analysis (Figure 3-3B), micro-Raman spectra (Figure 3-4 E), and XPS analysis (Figure 3-4 A-D) showed the variation in the topography and chemical properties of the created CNRN platform. Furthermore, the change in the scanning speed contributes to the variation in CNRN dosage on the substrate surface.

\subsubsection{Cytophilic Behavior.}

The graphite substrate consisting of stacks of parallel 2D graphene sheets (a single carbon layer in the crystalline honeycomb graphite lattice) has elemental carbon in the lowest energy state at an ambient temperature and pressure. ${ }^{[3-21]}$ The plain, smooth topography with the $\mathrm{C}-\mathrm{C}$ bond chemistry failed to generate enough favorable cues for the initial attraction to the cells, and hence the fibroblasts and HeLa cells did not attach to the substrate surface, as evident from the in vitro study of the fibroblast and HeLa cell seeding on the pure graphite surface (Figure 3-5). It was observed that the change in the CNRN indices of CNRN platforms has a significant impact on the modulation of cellular attraction of both fibroblasts and HeLa cells. As the CNRN index of the nanonetwork increases, both the cell lines showed an increasing trend in cellular attraction (Figure 3-6). Cell affinity to the CNRN platform might be due to a change in the physical and chemical aspects of the nanonetwork. Surface roughness always influences the cells, and at the same time, some type of cells might sense a change in topography well into the nanometer range. ${ }^{[3-55-3-57]}$ Again, the cell adhesion, viability, and morphological changes of HeLa and fibroblast cells are dependent on the level of protein adsorption on a modified surface. ${ }^{[3-39]}$ The results of previous studies showed that the surface of graphene was attractive to cells even in the absence ofFBS. ${ }^{[3-}$

${ }^{58]}$ The surface area plays a key role in the biological interaction with the nanomaterials. ${ }^{[3-59]}$ With a variation in the CNRN indices, the surface area exposure changes, and for the created CNRN 
platform, for a change in the CNRN index from low to high, there is an increase in the surface area exposure. With the expansion of the surface area, adsorption of protein increases, and hence, the CNRN platform as a nanostructured platform with an extended surface area showed favorable fate for both the cell lines to adhere and proliferate. ${ }^{[3-39-3-41]}$ A widely expressed family of transmembrane adhesion receptors, integrins, act as primary mediators in cell adhesion. ${ }^{[3-60]}$ Before cell adhesion begins, the nanostructured surface quickly adsorbs the serum proteins when it interacts with the FBS-containing culture medium. ${ }^{[3-61]}$ The type of extracellular matrix proteins (e.g., fibronectin, vitronectin, and albumin) that were adsorbed on the nanostructure and the strength of adherence to the surface modulate the subsequent cell adhesion. ${ }^{[3-62]}$ The CNRN platform might adsorb these cell-adhesive serum proteins in such an appropriate amount with a favorable conformation such that cell adhesion receptor integrins are able to form focal adhesions or multiprotein complexes, resulting in positive cell adhesion. ${ }^{[3-63,3-64]}$ Depending on the physicochemical property of absorbance, in this case of the CNRN platform, with the increase in the CNRN indices from low to high, more protein adsorbed resulted in an increment in cell adhesion and proliferation. One of the focal adhesion proteins, vinculin, helps in creating linkages between integrins (cell receptors) and actin filaments (cell cytoskeleton) and plays an important role in initiating and establishing cell adhesion, cell shape formation, and development of the cytoskeleton. ${ }^{[3-65]}$ The presence of the $\mathrm{C}-\mathrm{O}$ chemical bond along with $\mathrm{C}-\mathrm{C}$ in the created $\mathrm{CNRN}$ platform might play a contributive role in determining the cellular responses of both fibroblasts and HeLa cells toward the CNRN platform. Recent studies have confirmed that, in an oxygenated environment, there is an enhancement in cell survival and proliferation. ${ }^{[3-66]}$ Another research group showed that a high density of oxygenated groups on the surface of a graphene oxide film could potentially promote cellular proliferation and spreading with minimal cytotoxicity. ${ }^{[3-17]}$ 


\subsubsection{Cytotoxic Behavior.}

Recent literature data demonstrated that the exposure to graphene family materials such as graphene oxide could produce cytotoxicity in dose- and time dependent means. ${ }^{[3-67,3-68]}$ Again, the exposure environment (i.e., whether or not aggregation occurs) and the mode of interaction with cells are also factors for the toxicity of graphene and graphene oxide. ${ }^{[3-69]}$ According to our results, we might suggest the possible cytotoxic behavior mechanism of CNRN clusters on HeLa cells as follows: CNRN clusters when attached to the surface of HeLa cells provide an impetus to the cells that transduce inside of the cells and create de-regulation of adhesion-associated genes and corresponding adhesive proteins. Thus, the cells might detach and shrink in size. At the same time, CNRN clusters that enter into the cytoplasm by endocytosis pathway (Figure 3-11) might affect the energy metabolism of the cells. Again, as time elapses, the $\mathrm{C}-\mathrm{O}$ bond might create some sort of stress to the cells, which might disturb the gene transcription and translation and finally result in cell rounding, that is, apoptosis-like function.

\subsection{Summary}

Carbon nanomaterials/nanostructures have recently emerged as a new potential biomaterial for use in cancer research. These promising nanostructured platforms offer extensive drug delivery and cancer therapy applications. In this paper, we report the synthesis of a novel interwoven, bioactive CNRN platform that has a nano $\mathrm{C}-\mathrm{C}$ and $\mathrm{C}-\mathrm{O}$ molecular bond architecture that can attract fibroblasts and HeLa cells for adhesion and proliferation, with a time-dependent apoptosis-like function on HeLa cells and a cytophilic effect on fibroblasts without further chemical or biological functionalization. Single step ultrafast laser processing of the non-bio responsive $\mathrm{C}-\mathrm{C}$ bond containing a graphite plate under ambient conditions resulted in the surface ionization of graphite and the formation of a self-assembled bioactive CNRN with altered nanotopography and 
chemistry. The CNRN Index of the created CNRN platform varies from low to high with the increment of ionization energy from low to high. The nanochemistry of the created platform was composed of $\mathrm{C}-\mathrm{C}$ and $\mathrm{C}-\mathrm{O}$ bonds, and the variation in their percentage was achieved by controlling the ionization energy of the incident laser beam. Variation in the CNRN concentration on the graphite substrate surface was attained with the change in the scanning speed of the ultrashort laser processing of the substrate surface. Upon examination of the performance of the bioactive CNRN platform by exploring the response of normal fibroblasts and cervical cancer HeLa cells quantitatively and qualitatively, the results indicated that both fibroblasts and HeLa cells were attracted to the CNRN platform, and healthy attachment with robust growth was observed from the SEM and FM results compared with that of the native graphite substrate. The bioactive CNRN platforms actively accelerated the adhesion and proliferation with increased cellspreading areas for both the fibroblasts and HeLa cells. The number of cells that adhered and proliferated on the nanoplatform was 91 times more for the fibroblasts and 98 times more for the HeLa cells compared with the bulk graphite substrate, which is an indicator of the high proliferative nature of the created nanonetwork for both cell lines. Conversely, over time, the fibroblasts interlinked and developed tissuelike structures, whereas the adhered HeLa cells showed decreasing spreading areas, and the cells became round, developing a cocoonlike structure that had an apoptotis-like function, which might have led to cell death.

This in vitro study identified two distinct simultaneous functionalities of CNRN platform as the attraction characteristics of the extra cellular matrix (ECM) and the therapeutic attributes without the need of conjugation of any biomolecules/ drugs. CNRN platforms helped in the enhancement of cellular attraction and selective functionality through which fibroblasts exhibited tissuelike behavior and HeLa cells showed an apoptosis-like function. In this study, one mammalian and one 
cancer cell line have been used. Our future works will cover other fibroblast cell lines along with other cancer cells and corroborate the findings with them. 


\section{Chapter 4}

\section{SERS Active 3D Interconnected Nanocarbon Web Towards}

\section{Non-plasmonic In-vitro Sensing of HeLa and Fibroblast}

\section{Cells.}

Approved Pending Revisions in ACS Applied Materials and Interface, January 2018

Noninvasive technique of intracellular component analysis is important in early diagnosis and treatment of cancers. Carbon nanomaterials/nanostructures like CNTs, Graphene have low/no surface enhanced Raman scattering (SERS) ability. Due to low Raman response, they were conjugated with noble metals like gold/silver and attained SERS active detection of normal and cancer cells. Though carbon nanomaterials showed effectiveness in bio-molecule sensing through noble metal conjugation, their effectiveness was not explored individually as non-plasmonic SERS active platform for in-vitro cancer/normal cells detection. Here, for the $1^{\text {st }}$ time we introduce a unique non-plasmonic SERS based bio-sensing platform that uses biocompatible self-assembled 3D interconnected nanocarbon web (INW) for in-vitro detection and differentiation of HeLa and fibroblast cells. Self-internalization capacity resulted from sub-10 nanometer structure of bioactive INW along with label-free non-plasmonic SERS functionality attribute introduced live cell

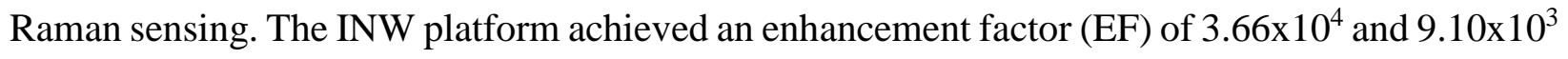
with crystal violet $(\mathrm{CV})$ and Rhodamine 6G (R6G) dyes respectively and it was significant in comparison to Raman enhancement factors on the surface of monolayer graphene (2 to 17). The results of time based Raman spectroscopy of both live HeLa and fibroblasts seeded on INW 
platform revealed chemical fingerprints of intracellular components like DNA/RNA, protein and lipids and their spectroscopic differences elucidate a guidance to specify each cell. The highest Raman signal enhancement achieved for intracellular components for fibroblasts was 4-fold and for HeLa was more than 5-fold. Furthermore, the spectral differences of SERS signal of the immobilized cell after 24 and 48 hours might shed light on cell health in presence of internalized INW clusters inside cells. The said unique attributes of INW platform were incorporated by a photon energy induced ionization brought with femtosecond laser that transform C-C bond of graphite to $\mathrm{C}-\mathrm{C}$ and $\mathrm{C}-\mathrm{O}$ bonds, and thus fabricated biocompatible INW platform. This simple label free, in-vitro diagnosis approach of HeLa and fibroblast have a huge potential for cancer research for medical bio-sensing.

\subsection{Introduction}

Carbon based nanomaterials gained considerable interest in the field of diagnostics and bio-sensing ${ }^{[4-1,4-2]}$. It is worth understanding the behavior of living cells and microorganisms immobilized on substrate while designing the biosensors ${ }^{[4-3]}$. Thus far, several optical bio-compatible methods such as infrared spectroscopy ${ }^{[4-4]}$, surface plasmon resonance ${ }^{[4-5]}$, bioluminescence imaging ${ }^{[4-6]}$ etc. have been applied for the investigation of cellular behavior in culture.

Researchers widely used Raman Spectroscopy which is a powerful, quick, label free and nondestructive investigation technique for biological analysis ${ }^{[4-7,4-8]}$. Raman analysis of live cells could provide useful biochemical information of cells. This information played an important role in identification of diseases, cell death, interactions of toxic agents or drugs with cells after treatment ${ }^{[4-9,4-10,4-11]}$. Despite several advantages the efficacies of Raman scattering process suffer, as it exhibits weak intensity ${ }^{[4-12]}$. The use of intense light sources, for example laser might be a solution however, it may have a negative consequence in cell investigations due to the degradation 
of the analyzed specimen. Thus, surface enhanced Raman scattering (SERS) technique utilizing nanostructured surfaces ${ }^{[4-13,4-14]}$ improve the sensitivity of traditional Raman spectroscopy while reducing the laser power and collection time ${ }^{[4-15]}$. With single molecular sensitivity SERS can be widely applied to chemical molecule/ion detection, clinical discrimination of cancer tissues etc. ${ }^{[4-}$ $16,4-17]$.

Noble metal nanostructures/nanomaterials such as gold, silver etc. have been mostly used as SERS materials because of their property of exhibiting localized surface plasmon resonance (LSPR) in the visible and near infrared (NIR) spectrum ${ }^{[4-18]}$. Both semiconductor hybrid nanostructures coated with monolayer noble metals ${ }^{[4-19,4-20,4-21]}$ and semiconductor-based nanomaterials, such as $\mathrm{TiO}_{2}{ }^{[4-15]}, \mathrm{ZnO}$, Graphene ${ }^{[4-22]}$ and $\mathrm{CuTe}^{[4-23]}$ have been reported to be SERS platform for various bio-sensing applications. Most current research is devoted to developing either noble metals or noble metal-coated semiconductor nanomaterials as SERS active substrates as noble-metal-based nanomaterials have demonstrated increased SERS sensitivity ${ }^{[4-24]}$. However, synthesis process such as chemical reduction, oxidation, or physical deposition used for creating noble metal and noble-metal-semiconductor hybrid nanomaterials have led to poor biocompatibility and making them undesirable for a variety of biomedical applications like biomolecular sensing materials ${ }^{[4-25]}$. Non-metallic carbon-based nanomaterials, such as carbon nanotubes $(\mathrm{CNTs})^{[4-26]}$, graphene ${ }^{[4-27]}$ and graphene oxides ${ }^{[4-28]}$ have been actively explored by the researchers in bio-sensing applications. Carbon based nanomaterials is capable of doing a direct electron transfer between the functionalized nanomaterials and active site of bioreceptor without involvement of any mediator, therefore it amplifies the signal as well as provide label free sensing ${ }^{[4-29]}$. Bio-sensing efficacy of pristine CNTs are influenced by its cytotoxicity as synthesized CNTs contain amorphous carbon and residue catalytic particles ${ }^{[4-30]}$. Covalent and non- covalent binding 
with different chemical groups completed the functionalization of CNT and makes CNTs biocompatible for conjugation with biomolecules and hence making them a suitable candidate for bio-sensing ${ }^{[4-29]}$. A limited/no effort was taken to utilize pristine CNT as SERS active substrate. Sun et. al. ${ }^{[4-31]}$ reported fabrication of SERS substrate through loaded the super aligned CNTs grid with densely packed Ag nanoparticles. They showed that in presence of R6G dyes the CNT grid alone did not depict any Raman enhancement. Lee et. al. ${ }^{[4-32]}$ also described the fabrication of a structurally tunable 3D SERS substrate based on vertically aligned CNTs and a high-density of gold nanoparticles on the walls of the vertically aligned CNTs. Ashwinkumar et. al. ${ }^{[4-33]}$ reported a technique for combining CNTs and portable Raman probes to effect simultaneous detection and photothermal ablation of ovarian cancer cells. Lipid-polymer coated Single-walled carbon nanotubes (SWCNTs) and multi-walled carbon nanotubes (MWCNTs) were used in this regard. So far, the graphene based biosensors are presented for sensing different molecules like enzymes, hydrogen peroxides, dopamine, and reduced b-nicotinamide adenine dinucleotide (NADH) ${ }^{[4-27,4-}$ ${ }^{34]}$. M. Manikandan et al. ${ }^{[4-13]}$ prepared two different SERS substrates by in situ synthesis of Au nanohexagons on Graphene templates and ornamentation of Au nanoparticles on the graphene sheets via electrostatic interaction using ultrasonication. These were applied to enhance Raman scattering and to differentiate human breast normal, cancer and cancer stem cells. Shijiang He et. al. ${ }^{[4-35]}$ developed a SERS-active substrate based on gold nanoparticle decorated chemical vapor deposition (CVD)-growth graphene and used it for multiplexing detection of DNA. Besides, Graphene and its derivatives (specially graphene oxide) conjugated with numerous materials such as conducting polymers, proteins, noble metals and act as bio-sensing platform for detection of platelet derived microparticles ${ }^{[4-28]}$, Escherichia coli $\left(\right.$ E. Coli) ${ }^{[4-36,4-37]}$ and the human papillomavirus DNA ${ }^{[4-38]}$. 
Thus far, the researchers used carbon nanomaterials as a component of hybridized bio-sensing platform along with noble metal nanoparticles such as $\mathrm{Ag}$ and $\mathrm{Au}$ and attained SERS active detection of different normal and cancer cells. However, carbon nanomaterials have not been reported as an individual biocompatible non-plasmonic SERS active bio-sensing platform for detection of normal and cancer cells in-vitro. Overall, carbon nanomaterials/nanostructures employed so far as SERS active bio-sensing platforms are in supportive role with noble metal nanoparticles. To the best of our knowledge carbon nanomaterials/nanostructures were not individually applied as a SERS based platform for in-vitro live cell diagnosis and differentiation due to their low/no SERS ability and/or toxicity. There is a need for biocompatible SERS active carbon basic platform without plasmonic nanoparticle addition which is being uptake by cells and help localized Raman detection of intracellular components and thus the differences of the Raman signature aid the detection of cells (whether cancerous like HeLa or mammalian like fibroblast). In the present research, we developed a SERS active, biocompatible 3D interconnected nanocarbon web structure and introduced a label free non-plasmonic SERS based bio-sensing platform for in-vitro detection and differentiation of HeLa (cancerous) and fibroblast (mammalian) cells. In our previous study, ${ }^{[4-39]}$ it was observed that the created nanocarbon platform possesses great biocompatibility and cell adhesion capacity. SERS activeness of the created INW platform was recognized with both crystal violet (CV) and Rhodamine 6G (R6G) chemical dyes. An enhancement factor of $3.66 \times 10^{4}$ and $9.10 \times 10^{3}$ were achieved for CV and R6G dyes respectively for the created INW structure in comparison to a bulk graphite substrate. This was significant in comparison to Raman enhancement factors on the surface of monolayer graphene (2 to 17) obtained in other literature ${ }^{[4-40]}$. Both SERS activeness of the created INW platform and endocytic uptake of INW clusters by both HeLa and fibroblast cells (due to sub-10 nanometer structure of 
the INW) provided SERS based Raman spectroscopy of live cells. The Raman SERS spectra collected from the live fibroblast and HeLa cells seeded on the INW platform containing samples during the 24 hours incubation period at a 6-hour interval depicted peaks related to intracellular biochemical components like proteins, DNA/RNA and lipids. Dominance of specific peaks in the Raman spectra profile and the significant spectral differences obtained for fibroblast and HeLa for live condition suggested a guideline in detecting and differentiating the fibroblast and HeLa cells. The highest Raman signal enhancement achieved for intracellular molecules for fibroblasts was 4fold and for HeLa was more than 5-fold. The differences in the spectral profile obtained for immobilized cells adhered on INW platform for 24 and 48 hours shed light on the cell health. The Raman spectra of immobilized fibroblast after 24 hours with a lot of DNA, protein and lipid peaks with moderately stronger signals might be an indication of healthy cells. The corresponding SEM and fluorescence micrograph confirmed the healthy well stretched cellular morphology with overextended filopodia and actin filament of fibroblast cells. A more dominant Raman response with strong DNA, protein and lipid peaks were obtained after 48 hours. The supporting SEM and fluorescence micrograph depicted an interconnected overlapped tissue like body of fibroblast and indicated that there was no adverse effect of internalized INW clusters on the fibroblast cells. On the other hand, the 24-hour spectra of HeLa cells had prominent peaks of DNA/RNA, proteins and lipids. Corresponding SEM micrograph showed healthy well spread HeLa cells with short filopodial extension. But there was a huge difference in spectral profile for 48-hour sample with absence of some of the DNA/RNA, proteins and lipids peaks. SEM and fluorescence micrograph supported these change as it depicted that the number of cells on the nanocarbon platform was reduced and the elongated HeLa cells stopped growing and seemingly decreased in size, forming a round shaped cocoonlike structure. Therefore, the nanocarbon platform induced cytotoxicity with 
the passage of time on Hela cells. The unique attributes of self-assembled 3D INW structure were introduced by adopting bottom up approach using photon energy induced ionization of the high energy femtosecond laser.

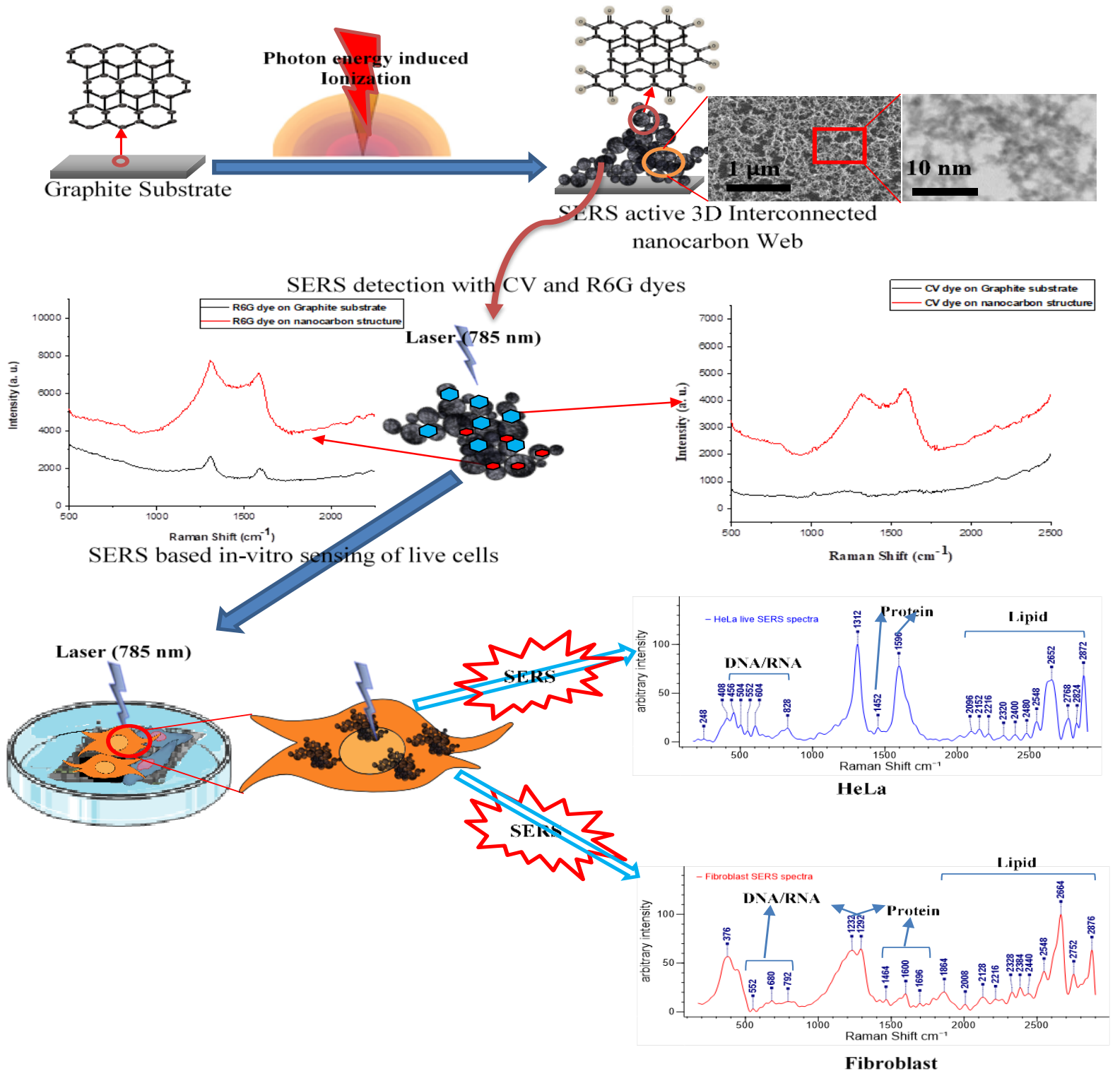

Figure 4-1: Overall graphical abstract showing the formation of SERS active 3D nanocarbon web, SERS detection with CV and R6G dyes and subsequent SERS based in vitro sensing of live cells. 
The ultrafast pulse laser ion-plume converted the $\mathrm{C}-\mathrm{C}$ bond of graphite to SERS active $\mathrm{C}-\mathrm{C}$ and C-O bonds bearing interwoven INW structure. The change in nanomorphology and nanochemistry of the created nanostructure is a function of adjustment of laser ionization parameters. The simplicity and versatility of the proposed SERS based INW aided Raman bio-sensing technique for HeLa (cancer) and fibroblast (mammalian) cell detection can be used for cancer diagnostics and or as a tool for evaluating new cancer therapy.

\subsection{Experimental Method}

\subsubsection{Interconnected nanocarbon web platform fabrication}

The 3D interconnected nanocarbon web platform was created on an isomolded, very fine grain, high strength graphite plate substrate (3 mm thickness) (Graphtek LLC, USA) through ionization with a diode-pumped, Yb-doped femtosecond laser system (Clark-MXR, Inc.; IMPULSE Series ultrashort pulse laser) at laser pulse repetitions of 4,8 and $26 \mathrm{MHz}$ and atmospheric condition. Before photon energy induced ionization processing, the plates were cut in to $4 \mathrm{~cm}^{2}$ squares, polished with sand papers (3M Canada, 1000 grit, 2000 grit and 3000 grit), ultrasonically cleaned (Cole-Parmer 8890 ultrasonic cleaner) with acetone and ethanol and then dried. An array of lines with varying space was machined on the samples mounted on a fixed stage with computer controlled high precision 2-D translation scanner guided the incident laser beam. Ultrashort pulse laser fluence (the pulse energy transferred to the substrate per unit area) varied at 4.43, 2.22 and $0.68 \mathrm{~J} / \mathrm{cm}^{2}$ (referred to as high, medium and low fluence) to alter the nano-topography and chemistry of the created interwoven nanocarbon network. However, the laser pulse width (214 fs) power of the incident laser beam $(15 \mathrm{~W})$, the laser beam scanning speed $(1 \mathrm{~mm} / \mathrm{s})$ and irradiation focal spot area $\left(84.62 \times 10^{-8} \mathrm{~cm}^{2}\right)$ were remained constant. 


\subsubsection{Morphological characterization of INW platform}

The surface morphology of the self-assembled 3D INW platform was evaluated initially with Scanning electron microscopy (SEM) (Hitachi SU-1500). The effects induced by the photon energy induced ionization on the fabricated 3D nanonetwork were studied and evaluated by the Field emission SEM (FESEM), Hitachi, SU-8200). Nanocarbon width distribution was determined through the analysis of images obtained from the FE-SEM, using Image $\mathrm{J}$ (image processing software).

\subsubsection{Physicochemical characterization of Nanocarbon platform}

Physicochemical properties of the fabricated bio-sensitive 3D INW platform were determined through the application of Micro-Raman spectroscopy and X-ray photoelectron spectroscopy (XPS). Both Micro-Raman spectroscopy and XPS analysis deduced the chemical composition of the INW platform. In addition, Micro-Raman spectroscopy also inferred crystallinity information of the created nanostructure.

A B\&W Tek, Inc NanoRam ${ }^{\circledR}$ handheld Raman system (wavelength $785 \mathrm{~nm}$ at a power of $350 \mathrm{~m}$ W) was used to examine both graphite substrate and developed interwoven 3D INW structure to identify how the created nanostructures changes the Raman spectra compared to the unablated substrate in terms of Raman intensity as well as any structural/compositional changes due to ionization process. The SERS analysis of the 3D INW platform was carried out with two dyes namely Rhodamine $6 \mathrm{G}$ and Crystal Violet to identify the SERS enhancement factor of the created INW network. These dyes due to their large Raman cross-section are popular for SERS analysis. Each dye was coated onto individual nanostructure areas at concentration of $1 \times 10^{-3} \mathrm{M}$ prior to Raman analysis. Each resultant Raman spectra was obtained at 3s collection time and repeated in triplicate then averaged. 
A Thermo Fisher K-Alpha XPS system using a monochromated Al Ka X-ray source with a 2:1 ellipse spot size of $400 \mathrm{~mm}$ as the major axis was employed to collect the XPS data. $1 \mathrm{~cm}^{2}$ samples were irradiated with the laser system and used for XPS analysis. To give better energy resolution and peak shape definition regional scans were performed at $50 \mathrm{eV}$ pass energy and $0.1 \mathrm{eV}$ point spacing. The Avantage software was used for elemental quantification of the created nanocarbon structure.

\subsubsection{Cell culture}

The functionality of the fabricated 3D INW platform in sensing different biological cells with the aid of micro Raman system was accomplished using mouse embryonic fibroblasts (NIH-3T3, ATCC, USA) and cervical cancer cells (HeLa, ATCC, USA). These cells were grown in Dulbecco's modified Eagle's medium (DMEM)/F12 supplemented with 10\% fetal bovine serum (FBS) and $1 \%$ penicillin/streptomycin, and the cultures were incubated at $37{ }^{\circ} \mathrm{C}$ in a humidified $5 \% \mathrm{CO}_{2}-95 \%$ air atmosphere.

\subsubsection{Fibroblast and HeLa Cells seeding on nanocarbon Platform}

Prior to in-vitro investigation, INW platforms were exposed to UV light for $20 \mathrm{~min}$ for sterilization. Subsequently, the platforms were placed inside Petri dishes containing $3 \mathrm{ml}$ of DMEM/F12 medium and $10 \%$ FBS per dish, and the cells were seeded at a density of $10^{5}$ cells $/ \mathrm{ml}$. The Petri dishes were placed in an incubator for 24 or $48 \mathrm{~h}$. During the incubation period, Raman spectra of the live cells along with the nanostructures were collected at the interval of every 6 hours $(6,12$, 18 and 24 hours) using the B\&W Tek, Inc NanoRam® handheld Raman system. The samples were then fixed with $2 \%$ glutaraldehyde after removing the spent medium. Subsequently, the samples were washed twice with $1 \%$ sodium cacodylate buffer $(\mathrm{pH} 7.3)$ at $4{ }^{\circ} \mathrm{C}$. Then, the cells were dehydrated through a graded ethanol series (from 10 to $100 \%$ ) for $15 \mathrm{~min}$ and were critical point 
dried. Raman spectrum of fixed samples were also collected for 24 and 48 hours. The Raman spectra at single cell (fixed) level was collected with Renishaw Raman microscope system (785 nm wavelength, 40X objective lenses). For SEM examination, the samples were sputtered with a gold layer. With a Hitachi SU1510 scanning electron microscope, the cellular morphology of the cells seeded on the samples was observed. To characterize nanocarbon uptakes caused by endocytosis, the energy-dispersive X-ray (EDX) mapping of these samples was carried out using Oxford EDX.

\subsubsection{Fluorescence microscopy of Cells.}

The samples were first fixed in methanol-free paraformaldehyde and then, incubated in skimmed milk to prevent nonspecific binding. Further incubation of the samples was then performed using Alexa Fluor 488 phalloidin (Life Technologies) to stain the actin and the cytoskeleton, followed by DAPI (Life Technologies) to stain the nucleus. The samples were studied using an epifluorescent Nikon E-400 microscope with a fluorescein isothiocyanate (FITC) and DAPI filter. The data were recorded using a DS-5M-U1 color digital camera (Nikon, Canada).

\subsubsection{Statistics}

Experiments were carried out in triplicate, and the data represented the mean \pm standard deviation, unless otherwise mentioned. The cell counting was carried out using SEM images and image processing software. One-way analysis of variance (ANOVA) was used to evaluate the statistical significance, with $* p<0.05$ and $* * p<0.01$ suggesting significant difference. 


\subsection{Results}

\subsubsection{Fabrication of 3D interconnected nanocarbon web(INW) platform}

The schematics in Figure 4-2 illustrates the synthesis of 3D interconnected nanocarbon web platform. The photon energy induced ionization which was brought by one step femtosecond laser processing on the surface of a graphite plate under ambient atmosphere synthesized the selffunctionalized 3D INW structure. With the precise guidance of a computerized galvano scanner the $2 \mathrm{D}(\mathrm{X}-\mathrm{Y})$ movement of laser beam transferred a predesigned array of lines onto the surface of the graphite sample. Femtosecond laser processing of the graphite sample assisted with megahertz pulse repetition rate caused surface ablation, nanoparticle evaporation and formation of vapor plume. In the dense vapor plume, there are different forms of carbon namely carbon ions, carbon radicals and neutral carbon ${ }^{[4-41]}$ and air molecules containing oxygen ions. The plume expanded into the surrounding air and its temperature dissipated and thus process of nucleation occurred and created self-assembled carbon nanoparticles containing both $\mathrm{C}-\mathrm{C}$ and $\mathrm{C}-\mathrm{O}$ bonds in the plume. Later upon collided and aggregated of these nanoparticles interwoven nanostructures of 3D interconnected nanocarbon web was formed. Complex dynamics of plume expansion as well as substrate property, ambient air vapor density and ionization energy influence the nanotopography and the altered nanochemistry of the fabricated platform.

There is a direct correlation between the ionization energy and the different laser parameters such as the laser power, ultrashort pulse laser fluence, laser repetition, and beam scanning speed. The nanocarbon dosage $\left(\rho_{n s}\right)$ is proportional to the number of ionized carbon nanoparticles $\left(N_{n p}\right)$ per pulse and it is directly proportional to the ultrashort pulse laser fluence, $f\left[\mathrm{~J} / \mathrm{cm}^{2}\right]=$ $E_{p}[J] / A_{f}\left[\mathrm{~cm}^{2}\right]$, where $E_{p}$ is the laser pulse energy and $A_{f}$ is the effective laser beam area. Again the said dosage is inversely proportional to laser machining speed ${ }^{[4-42]}, \rho_{n s} \propto \frac{1}{S}(f)^{1 / 2}$. For this 
study, we used three different ultrashort pulse laser fluence of 4.43, 2.22 and $0.68 \mathrm{~J} / \mathrm{cm}^{2}$, along with beam scanning speed of $1 \mathrm{~mm} / \mathrm{s}$ to achieve variation in the nano-topography and chemistry of the created nanocarbon structure.

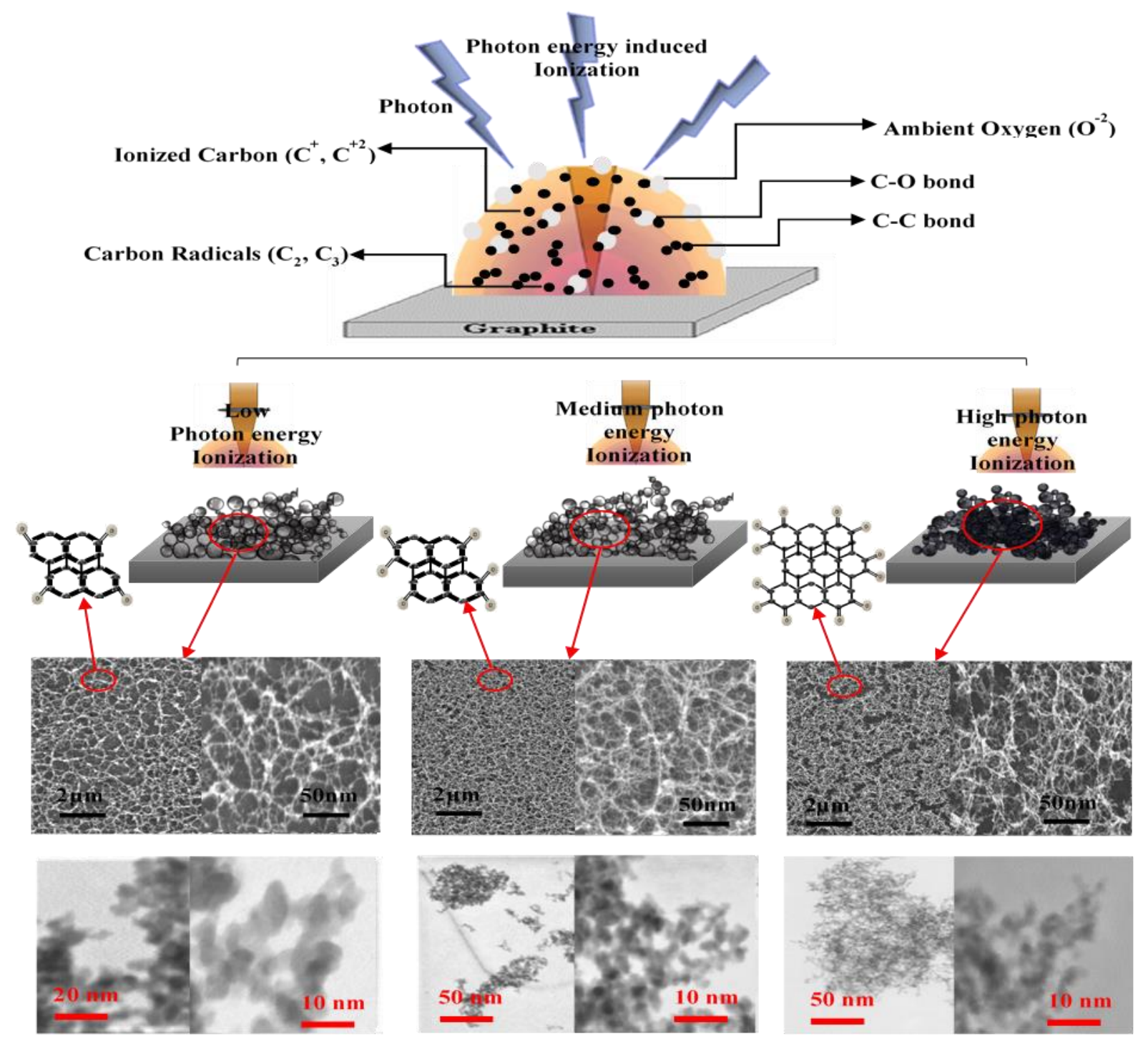

3D interconnected nanocarbon web Platform

Figure 4-2. Schematic illustration depicting the one-step synthesis of the 3D interconnected nanocarbon web platform. The physical morphology and the $\mathrm{C}-\mathrm{O}$ bond ratio of the nanocarbon structure accurately modulated with the change in ionization energy. 


\subsubsection{Morphology and physicochemical characterization of 3D INW platform}

With the help of ionization energy attained through femtosecond laser fluence irradiation, there is an evaluation of 3D web-like interconnected nanocarbon structure on the native graphite surface (Figure 4-3 A). With the increase in ionization energy from low to high, less to more compact nanofibrous structures were synthesized which was evident in the SEM micrographs (Figure 43A). The FE-SEM micrographs of the fabricated INW platform (Figure 4-2) also confirmed the interconnected nature of the nanoparticles. The graphite substrate contains basically $\mathrm{C}-\mathrm{C}$ bonds. Both physical morphology and alteration of chemical structure of the created nanostructure have been achieved through careful adjustment of laser processing parameters. The nanocarbon width and the $\mathrm{C}-\mathrm{O}$ to $\mathrm{C}-\mathrm{C}$ bond ratio describe the physical morphology and nano-chemistry of the 3D bio-sensitive nanocarbon platform (Figure 4-3 B). There is an opposite trend for these two parameters. With the increment of ionization energy from low to high, there is a gradual reduction of the nanocarbon width. However, the $\mathrm{C}-\mathrm{O}$ to $\mathrm{C}-\mathrm{C}$ bond ratio increases for the same gradual increment of ionization energy (Figure 4-3 B). A denser plume generated from higher ionization energy leads to a more compact nanocarbon structure.

The average size of the nanocarbon width varied from 6.67 to $5.27 \mathrm{~nm}$ with the increase in the ionization energy from 0.68 to $4.43 \mathrm{~J} / \mathrm{cm}^{2}$. the $\mathrm{C}-\mathrm{O}$ to $\mathrm{C}-\mathrm{C}$ bond ratios also change from 0.23 to 0.33 with the same increment of ionization energy and therefore termed as low, medium and high C-O concentrations. The frequency histograms of the nanocarbon width distribution (Figure 4-3 C) show that higher ionization energy tends to have a narrower width distribution. This observation agrees with our previous observation of the femtosecond laser synthesis of the Si nanostructure ${ }^{[4-}$

43]. The characteristic XPS spectra of the graphite substrate and the created 3D interconnected nanocarbon web platform for different $\mathrm{C}-\mathrm{O}$ concentration phases on the graphite substrate along 
with a quantitative assessment of the major elements are presented in Figure 2D. XPS spectra show two characteristic peaks at 285.08 and $533.08 \mathrm{eV}$ for the nanocarbon platform and the native graphite substrate, which can be ascribed to $\mathrm{C} 1 \mathrm{~s}$ and $\mathrm{O}$ 1s, respectively (Figure 4-3D). Both neutral and reactive gases, such as nitrogen and oxygen were present during the interaction of laser-mater in the presence of the surrounding atmosphere. Hence, $\mathrm{C}-\mathrm{O}$ bonds formed as reactive oxygen takes part in the chemical transformation with the carbon ions. Therefore, $\mathrm{C}-\mathrm{C}$ and $\mathrm{C}-\mathrm{O}$ bonds, two major chemical bonds were present in the created nano platform. The presence of oxygen in the nanocarbon platform increases with the increase in the ionization energy. From the quantitative assessment of the major elements (Figure 4-3 E) the $\mathrm{C}-\mathrm{O}$ to $\mathrm{C}-\mathrm{C}$ bond ratios are measured as 0.23 , 0.32 , and 0.33 at ionization energies of $0.68,2.22$, and $4.43 \mathrm{~J} / \mathrm{cm}^{2}$, respectively. The first peak position, which is at $285.08 \mathrm{eV}$ is interpreted as the $\mathrm{sp}^{3}$ hybridized carbon ${ }^{[4-44]}$. On the other hand, the peak position at $533.08 \mathrm{eV}$ interpreted as the $\mathrm{C}-\mathrm{O}$ bonds that correspond to the chemisorbed oxygen $^{[4-45]}$.

\subsubsection{SERS efficiency of 3D INW platform and EF calculation}

The Raman spectra of the graphite substrate along with the INW platform synthesized at three different ionization energies is depicted in Figure 4-4 A. The graphite substrate shows a G band $\left(\sim 1575 \mathrm{~cm}^{-1}\right)^{[4-46]}$ and a D band $\left(\sim 1350 \mathrm{~cm}^{-1}\right)$, the two known bands of graphite ${ }^{[4-47]}$. The bare graphite substrate has a crystalline lattice structure containing a defect on the graphene surface has been deduced from the presence of these two bands. The band peak position shifted to the range of 1578 to $1588 \mathrm{~cm}^{-1}$ and 1312 to $1320 \mathrm{~cm}^{-1}$ for the created INW platform. This peak shift could be the result of the addition of $\mathrm{C}-\mathrm{O}$ bond along with the $\mathrm{C}-\mathrm{C}$ bond in the created nanostructure. Another prominent peak was appeared approximately near $2700 \mathrm{~cm}^{-1}$. According to other

researchers, ${ }^{[4-48]}$ this peak is designated as $\mathrm{G}^{\prime}$, where $\mathrm{G}^{\prime}$-band appeared in the second order Raman 
spectra of crystalline graphite (without any kind of disorder) and corresponded to the overtone of the D-band. Based on the D and G band the intensity ratio of SERS active nanocarbon to graphite substrate were calculated and for both the Raman shift, there is a declining trend of these ratios with the increment of nanocarbon width (Figure 4-4B).
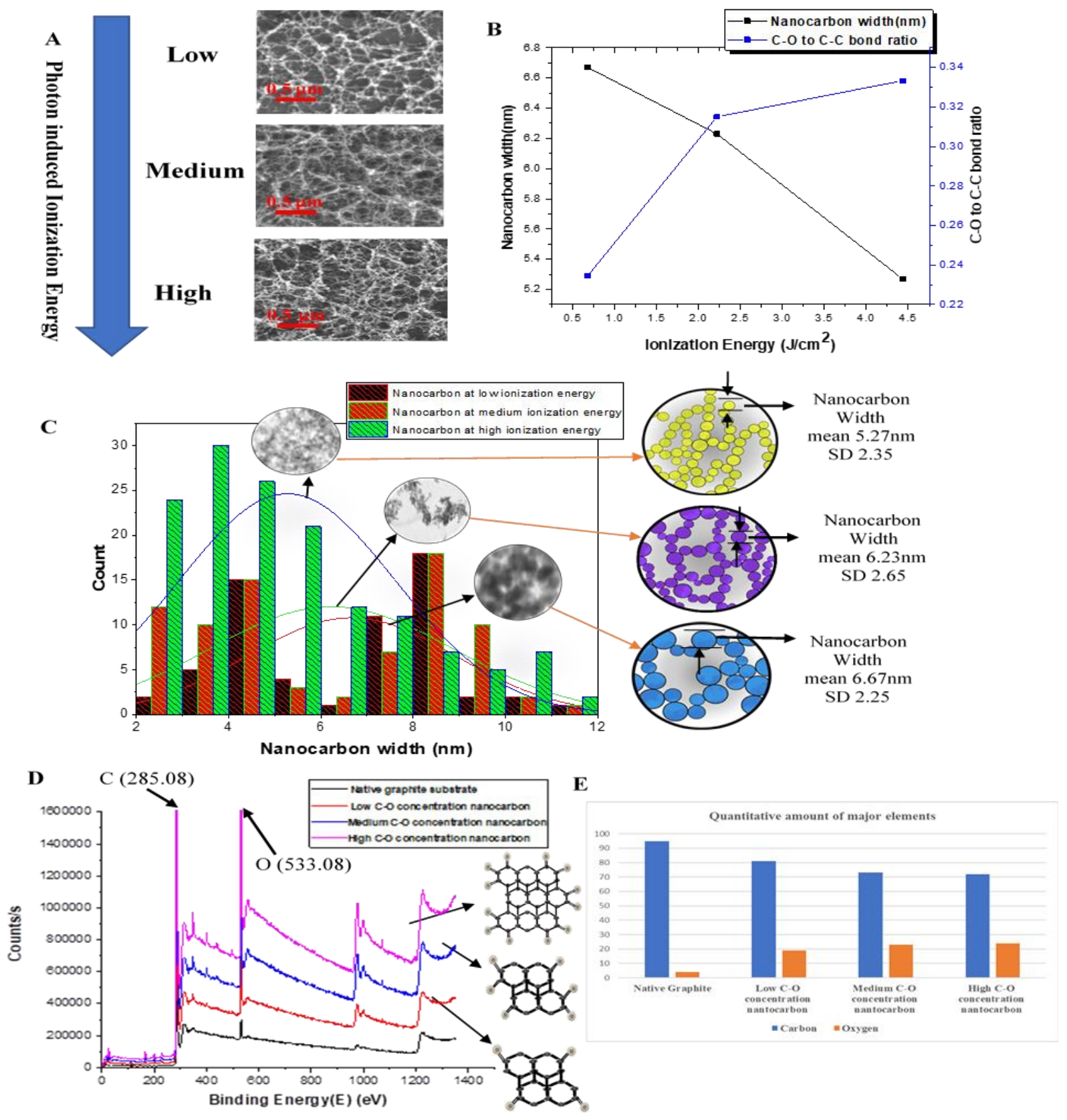
Figure 4-3 (A) Synthesized 3D interconnected nanocarbon web platform with (B) different nanocarbon width and $\mathrm{C}-\mathrm{O}$ concentrations phases at low, medium, and high ionization energies. (C) Nanocarbon width frequency histogram (D) XPS spectra of the nanocarbon with different C-O concentrations phases along with (E) corresponding quantitative assessment of the major elements.
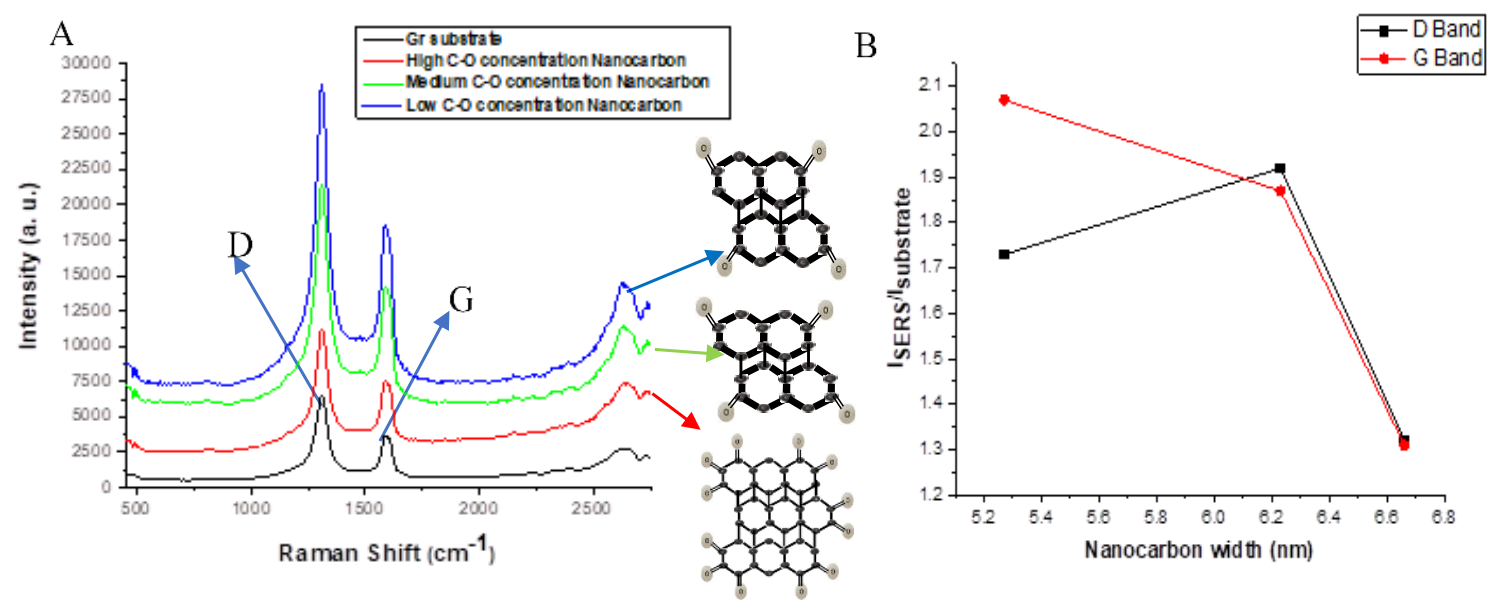

Figure 4-4. (A) Raman Spectra of the graphite substrate and the INW structure containing graphite substrate with different $\mathrm{C}-\mathrm{O}$ concentrations (B) $\mathrm{I}_{\mathrm{SERS}} / \mathrm{I}_{\text {substrate }}$ ratio at different nanocarbon width for $\mathrm{D}$ and $\mathrm{G}$ band

\subsubsection{Raman Enhancement factor $(\mathrm{EF})$ calculation}

Both crystal violet and Rhodamine $6 \mathrm{G}$ dyes were used to recognize the SERS enhancement factor of the 3D INW platform (Figure 4-5). These are popular dyes for SERS analysis due to their large cross section.

The details of the procedure used to determine the enhancement factor (EF) value of the nanocarbon platform is described in the Apendix 2. A significant enhancement in the intensity of the characteristic $\mathrm{G}$ band and D band Raman peaks of both dyes on the nanocarbon structure containing substrates of varies $\mathrm{C}-\mathrm{O}$ concentrations in comparison to that of the bulk graphite substrate was observed, which was evident from both the Raman spectra and EF values (Figure 45 A-D). It is detected that the bulk graphite substrate spectra have no or minimum response to the 
presence of a dye when they are coated with either the CV dye or the R6G dye. However, when a dye is coated onto the nanocarbon structure containing substrates, the characteristic peaks are clear and well defined.
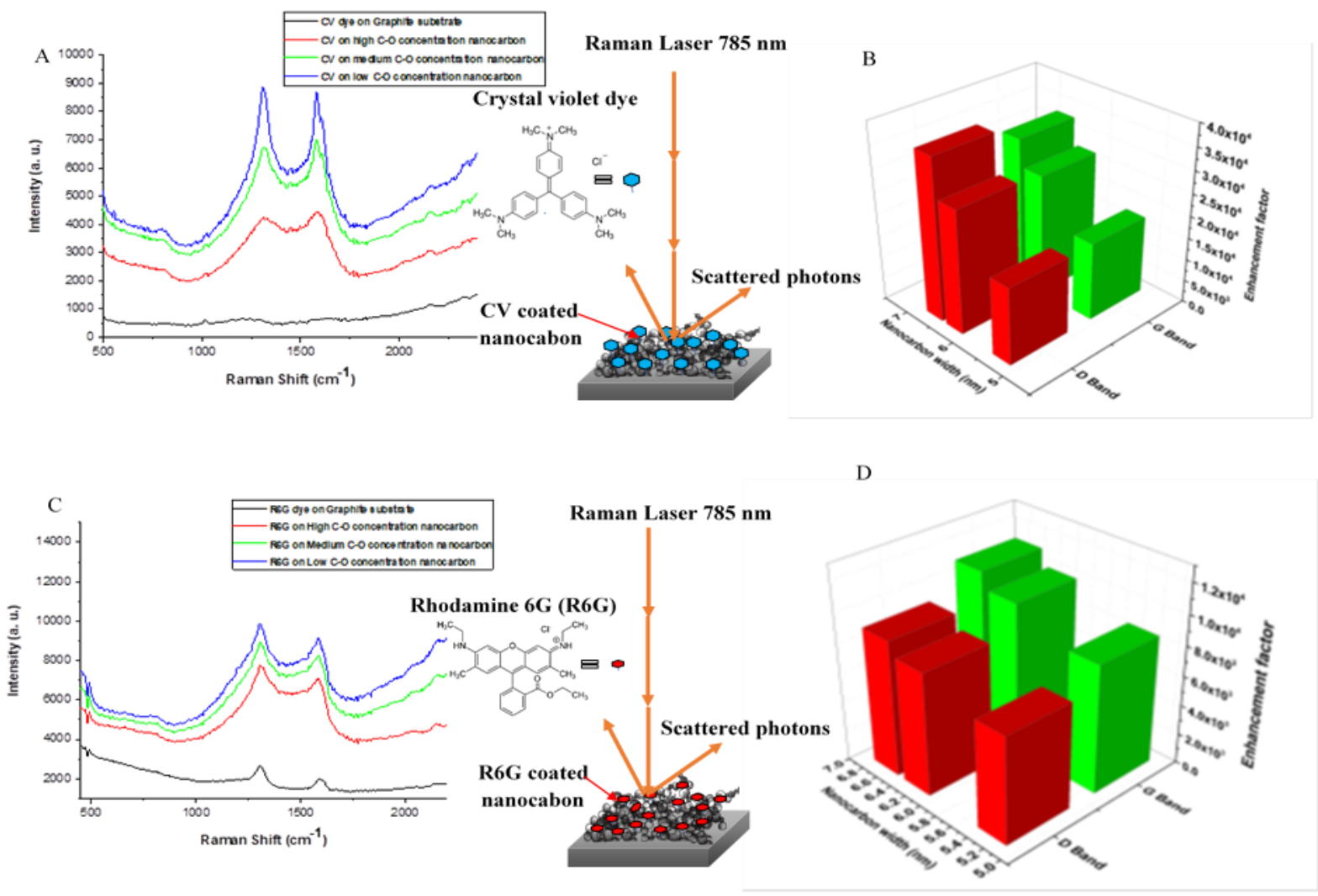

Figure 4-5. (A) Raman spectral enhancement with CV dye (B) Raman EF values with CV dye for $\mathrm{D}$ and $\mathrm{G}$ band at different nanocarbon width (C) Raman spectral enhancement with R6G dye (D) Raman EF values with R6G dye for D and G band at different nanocarbon width.

We observed a maximum enhancement of $3.66 \times 10^{4}$ and $9.10 \times 10^{3}$ for both $\mathrm{CV}$ and $\mathrm{R} 6 \mathrm{G}$ dyes respectively at $10^{-3} \mathrm{M}$ concentration. Highest $\mathrm{EF}$ values were achieved for higher nanocarbon width for both the cases which might be due to higher cross section area associated with higher nanocarbon width in comparison to lower nanocarbon width. Two mechanisms are considered for SERS enhancement phenomena ${ }^{[4-13,4-49]}$. These are electromagnetic mechanism (EM) and 
Chemical mechanism $(\mathrm{CM})$. The basis for EM is the enhancement of the local electromagnetic filed that results in a significant increase in the Raman scattering cross section. The surface plasmons excited by the incident light is the main contributor to the electromagnetic enhancement ${ }^{[4-50]}$. The enhancement factor for the EM enhancement can reach to $10^{10}$ or more ${ }^{[4-51]}$ In CM enhancement ${ }^{[4-52]}$, a new resonance state is generated through a charge transfer between the substrate and absorber. Here the cross section of the Raman scattering increases as the positive and negative charge in the molecule become more separated. CM enhancement offer an enhancement factor of 10 to $10^{2[4-40]}$. In our study, it is presumed that CM mechanism employed for Raman signal enhancement with an enhancement factor of $10^{3}$ to $10^{4}$ which is in the higher side in comparison to Raman enhancement factors on the surface of monolayer graphene (2 to 17) obtained in other literature ${ }^{[4-40]}$. Therefore, the nanocarbon platform is highly SERS active and can detect an analyte much more readily than the bulk graphite. 

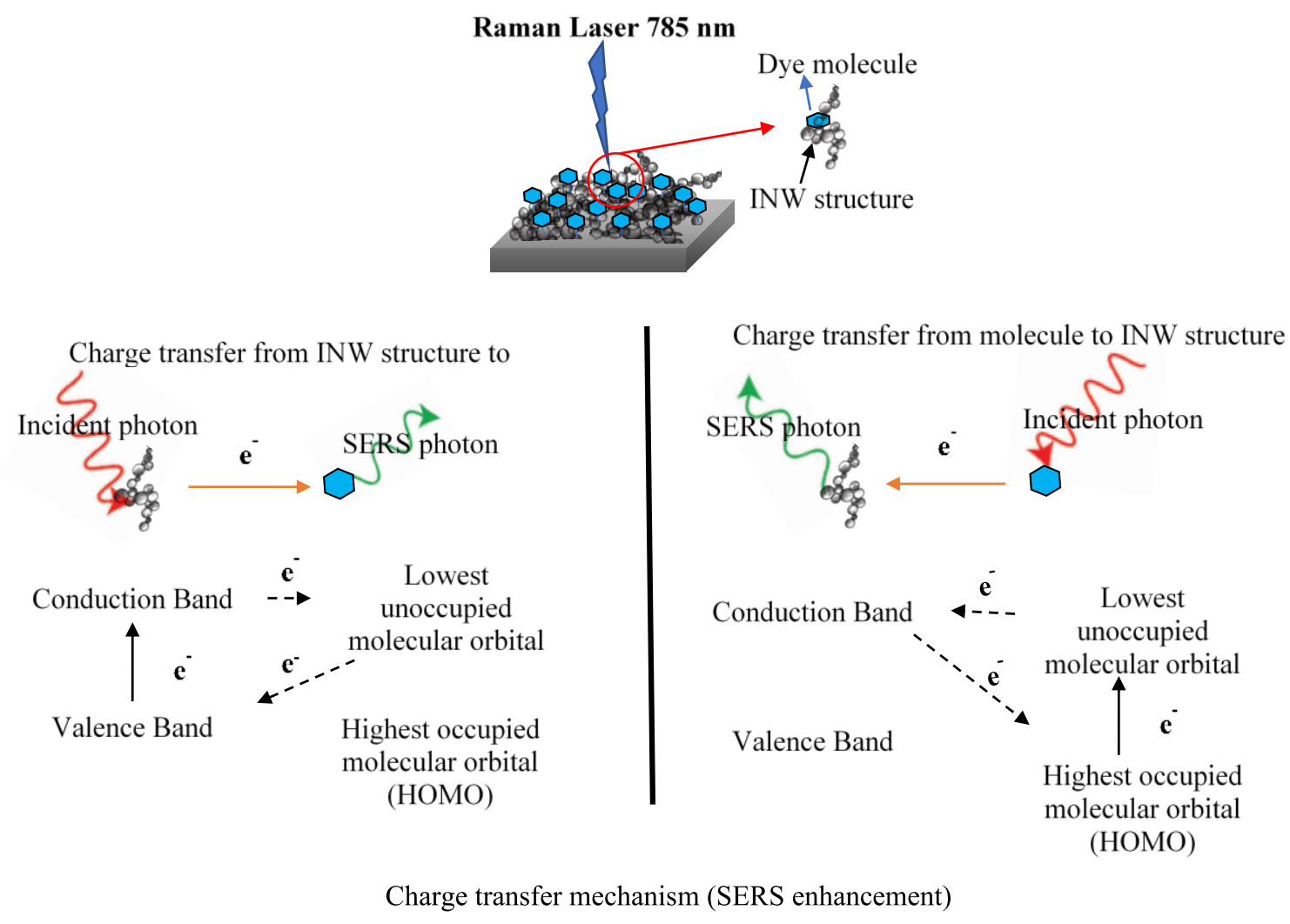

Figure 4-6. Schematic of the charge-transfer mechanism principle.

The SERS efficiency of 3D INW platform might be explained with CM enhancement. The schematics in figure 4-6 showed the principle of the charge-transfer mechanism for $\mathrm{CM}$ enhancement. $\mathrm{C}-\mathrm{C}$ bond is the core structure of the created INW platform. The CM mechanism requires charge transfer between the molecule and the substrate and the distance between the molecule and the substrate need to be below $0.2 \mathrm{~nm}^{[4-53,4-54]}$. The core C-C bond of the INW platform might induce charge transfer which was similar to graphene ${ }^{[4-55]}$. When an analyte molecule is in close proximity to an INW structure surface and is excited by a photon source the charge-transfer resonance happens ${ }^{[4-56]}$. The valence band $(\mathrm{VB})$ and conduction band $(\mathrm{CB})$ energy levels of the INW structure should be relatively comparable to the lowest unoccupied molecular 
orbital (LUMO) and the highest occupied molecular orbital (HOMO) of the dye molecule respectively. Electrons can travel along the showed path in the figure thermodynamically from either the INW structure to the molecule or vice versa if these energy levels are within the range of each other and the incident light has enough energy. When a photon excites an electron from the $\mathrm{VB}$ to the $\mathrm{CB}$, it is transferred to the LUMO through resonant tunneling because they are neighboring each other and have comparable energy level. This electron transfers energy to a vibrational state of the molecule and causes a SERS photon to be emitted from the molecule. Then it decays back to the $\mathrm{CB}$. Likewise, through resonant tunneling an electron might be excited by a photon from the HOMO energy level to the LUMO energy level and transferred to the CB of the INW structure. After transferring energy to the vibrational state of the molecule the electron will decay to the ground energy level of the molecule and thus causes emission of SERS photon.

\subsubsection{INW cluster uptake}

Cellular uptake provides necessary local optical fields for ultrasensitive intracellular probing ${ }^{[4-57]}$. Figure 4-7 portrayed INW cluster uptake process by both HeLa and fibroblast cells during the period of incubation. The results of SEM, fluorescence and EDX mapping were based on the high C-O concentration INW platform. The results for other two conditions were included in the supporting information section. Both SEM and fluorescence micrographs (figure 4-7 A) evidenced the presence of INW clusters inside the fibroblast and HeLa cells. When seeded, both fibroblast and HeLa cells had an affinity to attach to the areas of higher protein adsorption sites. Bio-sensitive INW platform being a higher protein adsorption area ${ }^{[4-58,4-59,4-60]}$ attracted the cells and primary attachment took place. It might be hypothesized that the internalization of INW clusters, a constructive components of the interwoven 3D INW platform, was occurred through endocytic uptake due to the contiguity of cells to the nanocarbon platform. The endocytic process of taking 
up INW nanoclusters by cells was determined by the nanoparticle size, shape, surface charge as well as type of cells ${ }^{[4-61,4-62]}$.

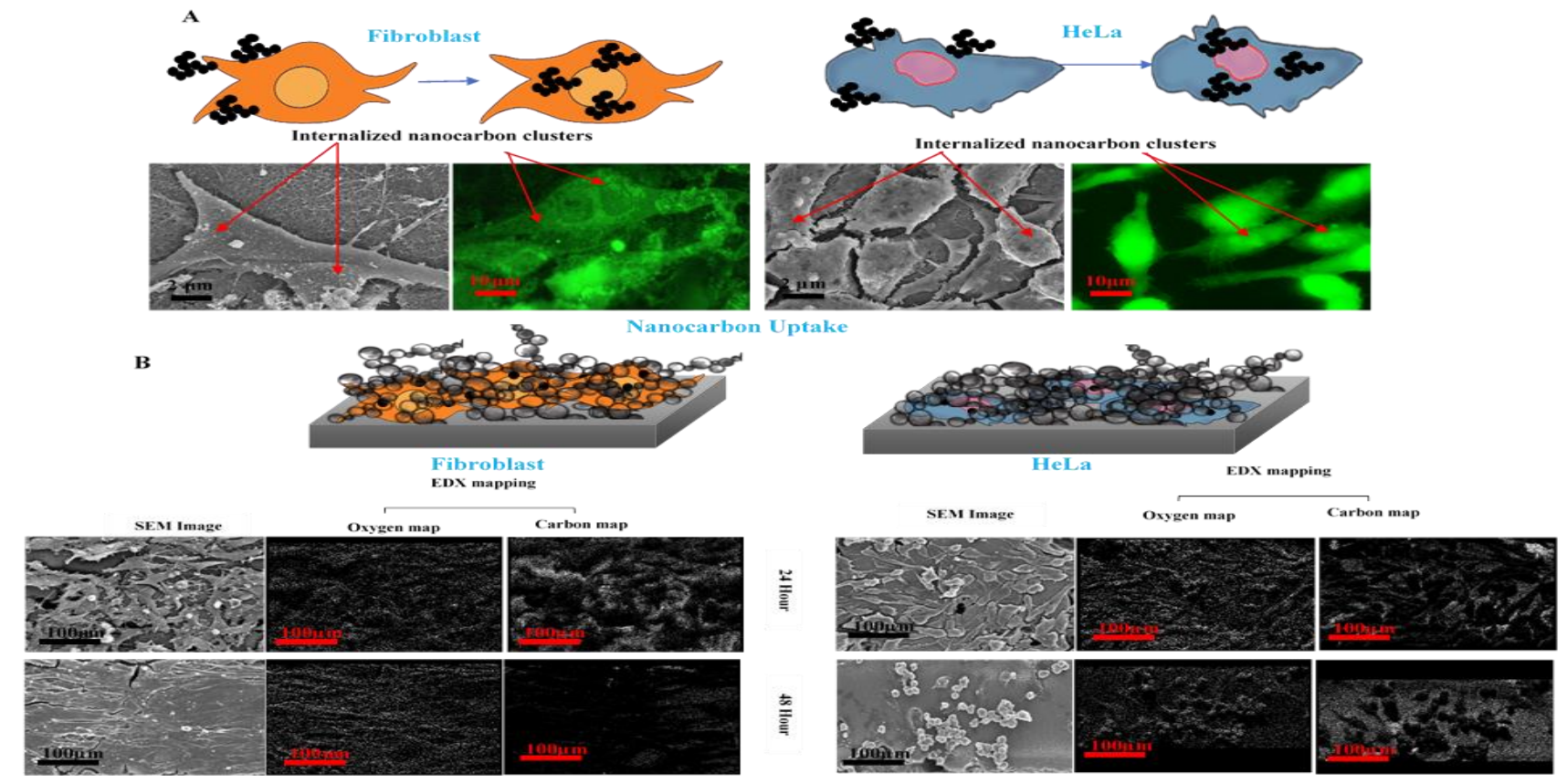

Figure 4-7 (A) INW cluster uptake by the fibroblast and HeLa cytoskeletons attached to the nanocarbon platform and (B) EDX mapping images of the Fibroblasts and HeLa cytoskeletons attached to the nanocarbon platform showed internalized INW clusters.

The presence of internalized INW clusters inside fibroblast and HeLa cells (figure 4-7A) evidenced INW clusters uptake by the endocytosis process ${ }^{[4-63,4-64]}$. EDX elemental mapping (Figure 4-7B) further elucidate the presence of INW clusters in the cell cytoskeleton. The spreading pattern of both carbon and oxygen mapping identified the presence of $\mathrm{C}-\mathrm{O}$ and $\mathrm{C}-\mathrm{C}$ bonds structures, the nano-chemistry of the nanocarbon platform, and thereby evident that there was internalization of INW clusters in both the fibroblast and HeLa cells.

\subsubsection{SERS Raman spectra of the live fibroblast and Hela cells}

An insight into the biochemical composition of fibroblast and HeLa cells could be achieved through acquiring Raman spectra from proteins, nucleic acids (DNA/RNA) and lipids which are 
the intracellular components of cells. Raman spectra of live cells (both fibroblast and HeLa) seeded on the INW platform were collected during the incubation period of 24 hours at an interval of 6 hours (Figure 4-8). The cells were seeded covering the entire sample which constitutes both INW structure containing graphite substrate and the native graphite substrate. The Raman spectra collected outside the nanostructure zone along with seeded cells was termed as native spectra. The Raman spectra after 24-hour incubation on high C-O concentration nanocarbon platform were chosen for spectral analysis.

\subsubsection{SERS spectra of live fibroblast cells}

During the first 6 hours of incubation the cells were looking for suitable site for adhesion. After 6hour, live cells response to Raman signal was significant. There was variation in peaks over time (24 hours of incubation) as the live cells undergone morphological changes over the mentioned period. The peak variation was dominant for the High C-O concentration INW structure containing SERS substrate. A tentative assignment of Raman peaks was obtained for both live fibroblast on high C-O concentration nanocarbon platform after 24 hours with reference to the earlier studies ${ }^{[4-}$ 65, 4-66] (Table S1 in Apendix-4a). Only the peaks related to the biological molecules were tabulated. From the Table S1, different intracellular molecules such as DNA $\left(680 \mathrm{~cm}^{-1}, 792 \mathrm{~cm}^{-1)}, 1292 \mathrm{~cm}^{-}\right.$

$\left.{ }^{1}\right)$, proteins $\left(1232 \mathrm{~cm}^{-1}, 1600 \mathrm{~cm}^{-1}, 1696 \mathrm{~cm}^{-1}\right)$, lipids $\left(1464 \mathrm{~cm}^{-1}, 2328 \mathrm{~cm}^{-1}, 2440 \mathrm{~cm}^{-1}\right)$, lipids $\left(1700 \mathrm{~cm}^{-1}-2900 \mathrm{~cm}^{-1}\right)$ and $\mathrm{CH}_{2}$ asymmetric stretch of lipids and proteins $\left(2876 \mathrm{~cm}^{-1}\right)$ were identified.

\subsubsection{SERS spectra of live HeLa cells}

In case of HeLa, the live cell response to Raman signal was also significant after 6-hour incubation period in comparison to other time intervals. Though there were peak variations over the specified 


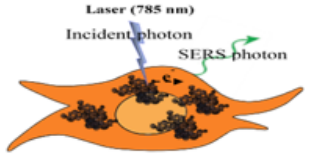

Fibroblasts
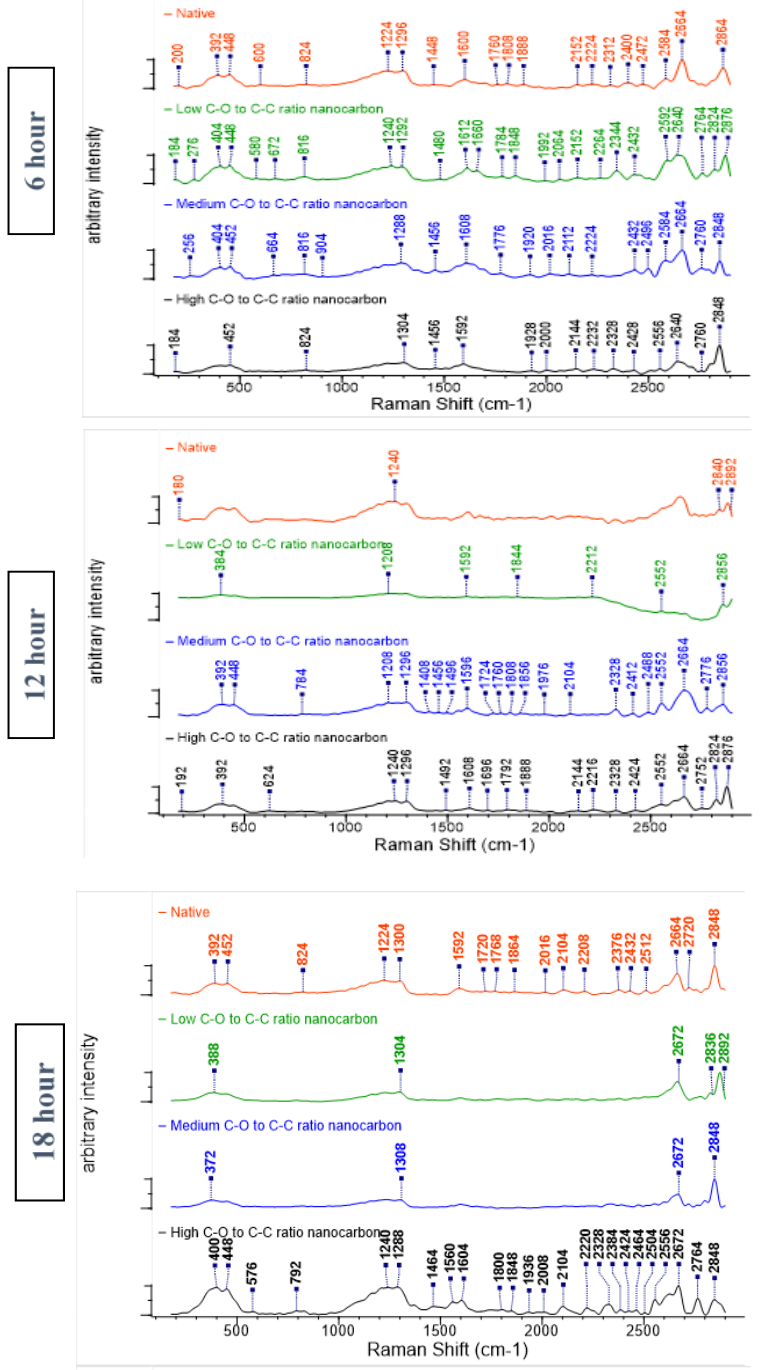

- Native

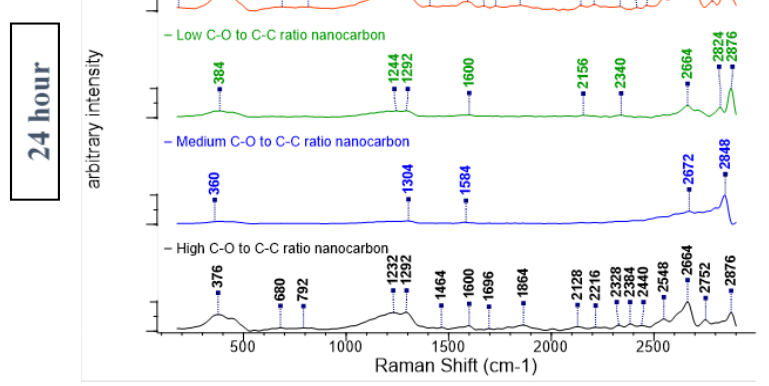

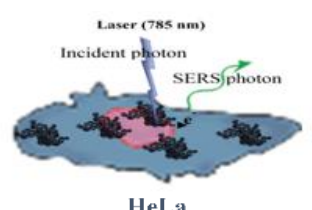

HeLa

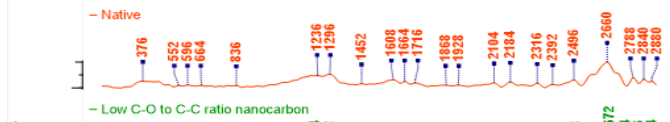

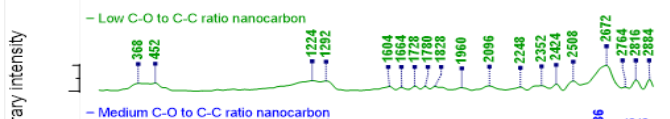

- Medium C-O to C-C ratio nanocarbon

] [U [L]

- High C-O to C-C ratio nanocarbon

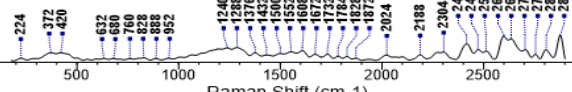

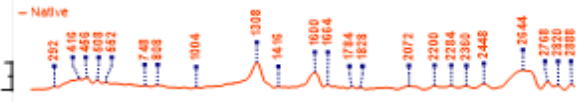
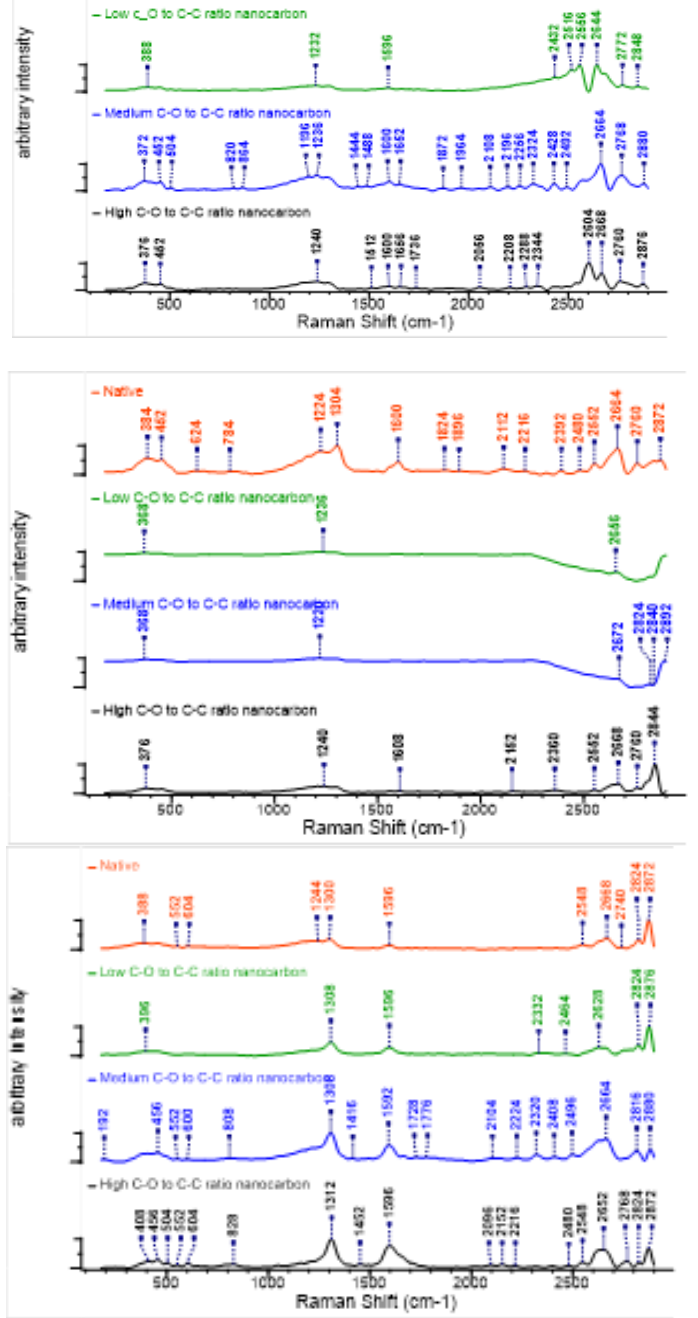

Figure 4-8 SERS Raman spectra of live fibroblast and HeLa cells seeded on different C-O concentrations INW platform for 24 hours incubation period with 6 hours intervals. 
time (24 hours incubation) for different $\mathrm{C}-\mathrm{O}$ concentrations nanocarbon structures containing substrate the peak was steady and prominent for high C-O concentration INW structure containing substrate. A similar tentative assignments of Raman peaks was also obtained for live HeLa cells on high C-O concentration nanocarbon platform after 24 hours (Table S2 in Apendix 4b). According to Table S2, the identified intracellular molecules were DNA/RNA $\left(456 \mathrm{~cm}^{-1}, 502 \mathrm{~cm}^{-}\right.$ $\left.{ }^{1}, 552 \mathrm{~cm}^{-1}, 604 \mathrm{~cm}^{-1)}, 828 \mathrm{~cm}^{-1}\right)$, proteins $\left(1452 \mathrm{~cm}^{-1} 1596 \mathrm{~cm}^{-1}\right)$, lipids $\left(408 \mathrm{~cm}^{-1}, 1700 \mathrm{~cm}^{-1}-\right.$ $\left.2900 \mathrm{~cm}^{-1}\right)$ and $\mathrm{CH}_{2}$ asymmetric stretch of lipids and proteins $\left(2872 \mathrm{~cm}^{-1}\right)$.

\subsubsection{Identification of Fibroblast and HeLa cells from live SERS Raman spectra}

Figure 4-9 depicted the gradual changes in SERS based Raman spectra of live HeLa and fibroblast cells for 24 hours incubation period with 6 hours interval. The Raman spectra after 6 hours incubation contained the peaks for DNA/RNA, Protein and lipids for both HeLa and Fibroblasts. There were no prominent changes in lipid peaks over the period for both the cells. However, both DNA/RNA and protein peaks were changes and HeLa and Fibroblast showed a significant variance in spectral profile (Figure 4-9). Two distinct dissimilarities were observed for both fibroblasts and HeLa cells. After 24 hours incubation DNA/RNA peaks were more prominent $\left(400 \mathrm{~cm}^{-1}\right.$ to 610 $\mathrm{cm}^{-1}$ range) for HeLa in comparison to Fibroblast. Conversely, the bundle of protein peaks of fibroblast (1200 $\mathrm{cm}^{-1}$ to $1700 \mathrm{~cm}^{-1}$ range) was dominant over HeLa cells. Therefore, based on the peak profile, DNA/RNA peaks for HeLa and protein peaks for fibroblasts might be the identifying features for differentiation for these cells.

\subsubsection{SERS effect on live Fibroblast and HeLa cells}

The Raman signal enhancement factor for the intracellular components like DNA/RNA, protein and lipids of both fibroblasts and HeLa cells was calculated to identify the effect of INW clusters 
on the Raman spectral quality. The Raman spectra acquired for live fibroblasts and HeLa cells after 24-hour incubation on high $\mathrm{C}-\mathrm{O}$ concentration nanocarbon platform and the corresponding

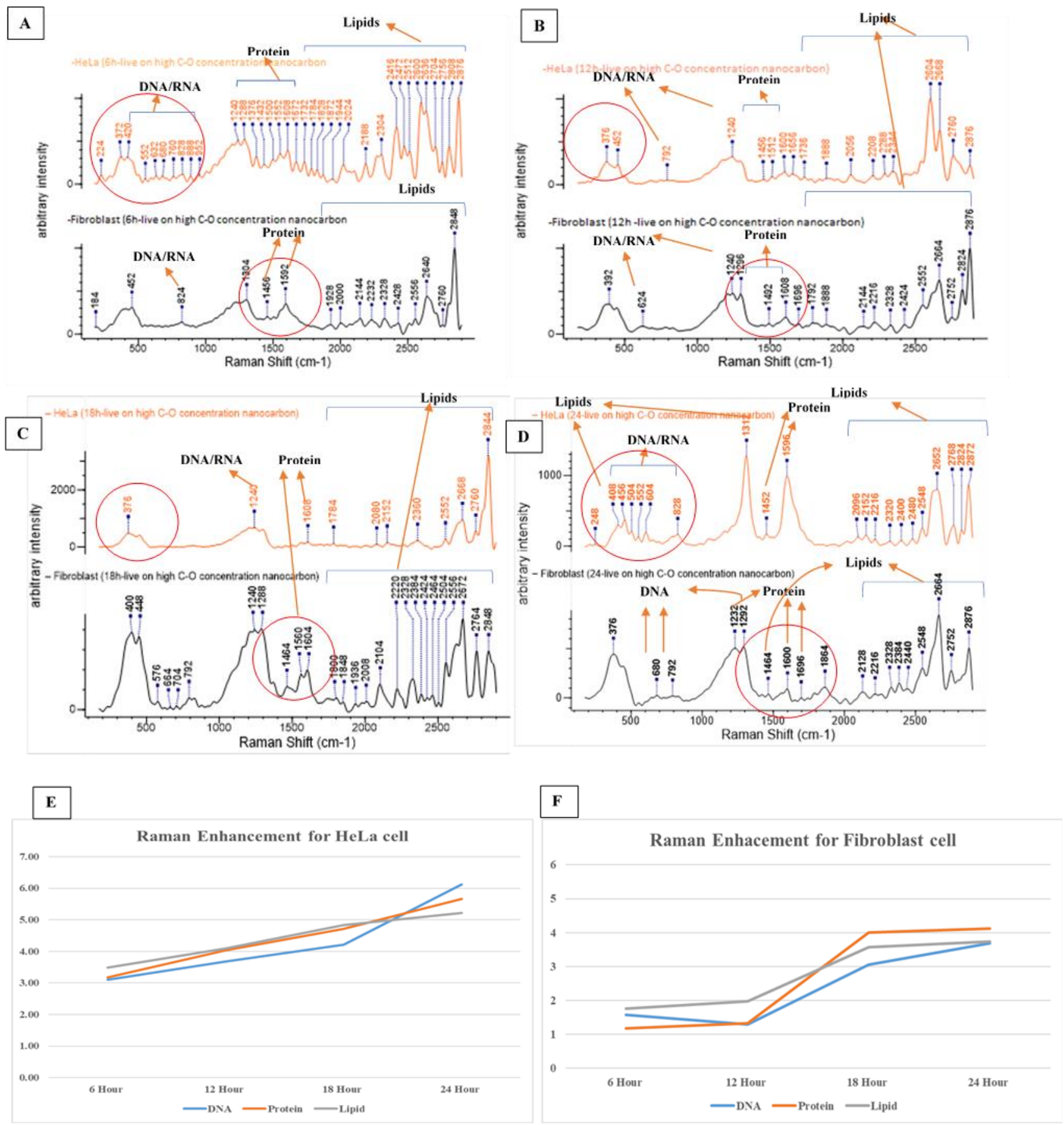

Figure 4-9 (A-D) Gradual changes in SERS Raman spectra for live Hela and Fibroblast cells on high C-O concentration INW platform acted as basis for cell differentiation (E) SERS based 
Raman signal enhancement of intracellular components of HeLa cells (F) SERS based Raman signal enhancement of intracellular components of Fibroblast cells

native spectra were taken for this purpose. The figure 4-8 (E, F) showed overall Raman enhancement factor graph for DNA, protein and lipid for HeLa and Fibroblast respectively. The results were tabulated in supporting information (Table S3 and Table S4 in Apendix 4c and d). For HeLa DNA showed highest Raman enhancement which was more than 5-fold. In case of fibroblast the protein showed 4-fold Raman enhancement. Therefore, there was a significant Raman signal enhancement achieved in presence of INW clusters.

\subsubsection{SERS Raman spectra of the immobilized fibroblast and Hela cells}

An in-depth study of the Raman spectroscopy of the immobilized fibroblasts and HeLa cells that adhered on the INW platform was carried out and analyzed along with the SEM and fluorescence micrograph of the same (Figure 4-10 A, B). Both fibroblast and HeLa cells containing samples were fixed after 24 and 48 hours and Raman spectra of these samples were carried out. The Raman spectra of native (the area outside the INW platform zone containing cell) after 24 hours showed very week signal with a few peaks. The Raman spectra of immobilized fibroblast cells adhered on the INW platform showed a lot of peaks with moderately stronger signals and with little variation for different C-O concentrations nanocarbon platforms. The Raman spectra of fibroblast after 48 hours was more prominent in comparison to the spectra obtained for 24 hours. Both the cases, the peaks were assigned as DNA/RNA, Protein, lipid and glycogen peaks. From the SEM micrograph, it was evident that the fibroblast cells were well-adhered, stretched, and had an extended cellular morphology with overextended filopodia and actin filament. The fluorescence micrograph is also in agreement with it. After 48 hours, these adhered fibroblasts turned to a tissue like structure 
because of growth enhancement. As the fibroblast cells turned to an interconnected overlapped tissue like body, the Raman response in presence of SERS active INW clusters was more dominant which was evident from the peaks of DNA/RNA, Protein, lipid and glycogen. Variation of C-O concentrations in the nanocarbon platform had little or no effect on the resultant spectra.

On the other hand, the native Raman spectra of the 24-hour and 48-hour samples of HeLa cells was too weak though some peaks were present in the spectra. The 24-hour spectra of HeLa cells adhered on the INW structure containing substrates of varying C-O concentrations was more prominent and had a handsome number of peaks of different intracellular biological molecules like DNA/RNA, protein, lipid and glycogen. However, a significant difference was observed for HeLa cell Raman spectra after the 48-hour incubation. Though there was a little spectral variance for low $\mathrm{C}-\mathrm{O}$ to $\mathrm{C}-\mathrm{C}$ ratio nanocarbon platform in terms of spectral peak but with the increment of $\mathrm{C}$ O to C-C bond ratio in the INW platform there was variation in both the strength of the signals and amount of Raman peaks. With the increment of C-O concentrations and nanocarbon width there was completed disappearance of DNA peaks. SEM micrograph of HeLa cells after 24 hours showed well adhered, well-spread and elongated cells with short filopodial extension and it was supported by the fluorescence micrograph. But from the 48-hour SEM micrograph, it was evident that the number of cells on the nanocarbon platform was reduced and the elongated HeLa cells stopped growing and seemingly decreased in size, forming a round shaped cocoonlike structure. The reduction in the number of cells and the morphological change of Hela cells overtime resulted in the change in Raman spectra.

Subsequently, a comparative Raman shift range wise analysis of spectral profile of immobilized fibroblast and HeLa cells for 24 and 48 hours was performed to help detection of cell health of the fibroblast and HeLa cell population with internalized INW clusters with the passage of time. The 
basis for this analysis was the assigned Raman peaks for immobilized fibroblasts and HeLa cells for 24 and 48-hour samples along with the SEM and fluorescence micrographs.

Kurt W. et al ${ }^{[4-67]}$ in their research detected biochemical changes due to proliferation in mammalian cell cultures with Raman spectroscopy. The assigned Raman peaks for both live and immobilized fibroblast cells (Table S1 in Apendix 4) represented the chemical signatures of Protein, lipid, DNA/RNA and glycogen. 

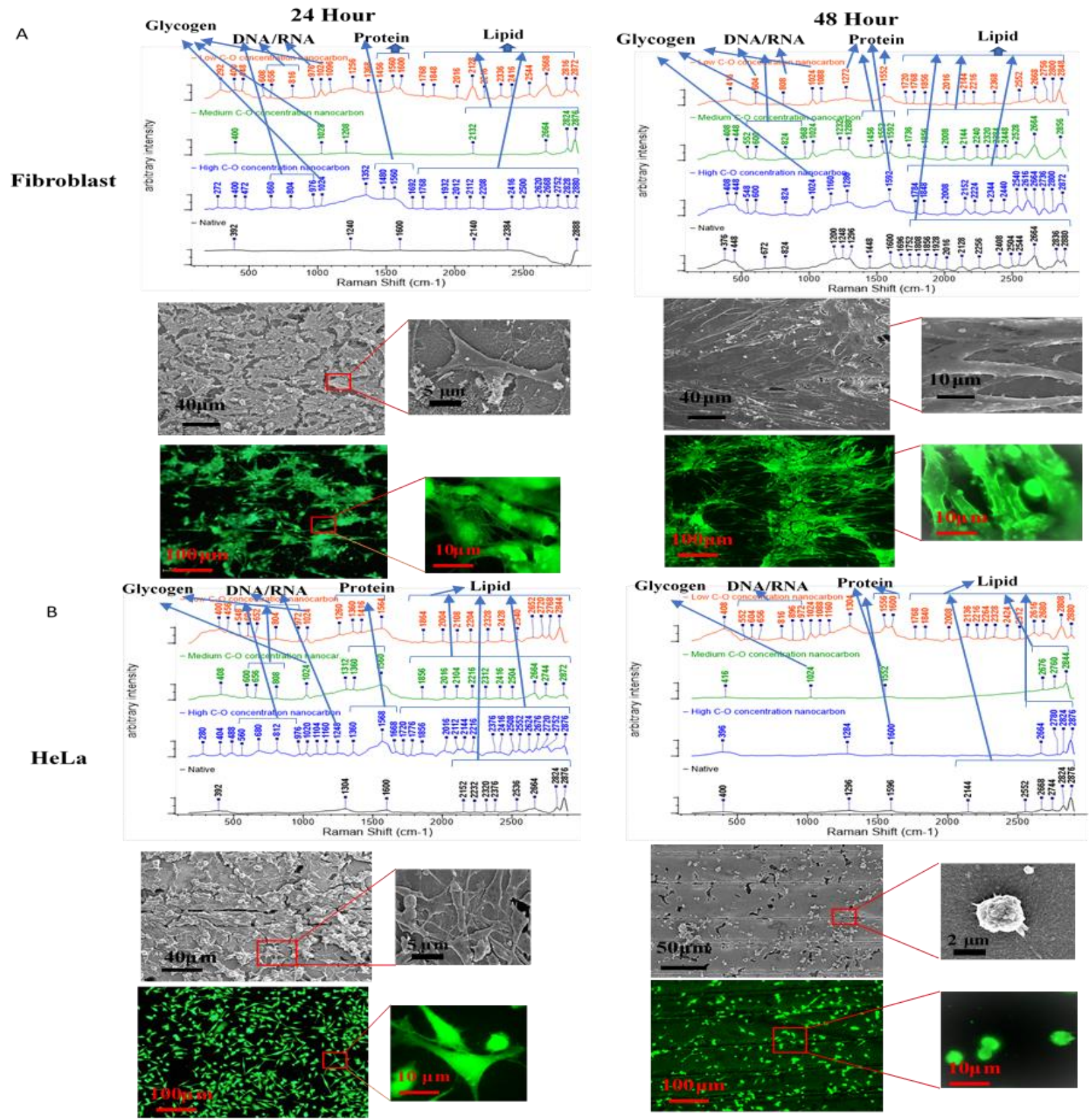

Figure 4-10 (A)Variation in different intracellular components peaks of immobilized fibroblast cells based on SERS Raman spectra obtained from 24 and 48 hours samples containing different C-O concentrations INW platforms along with SEM and fluorescence micrograph and (B) Variation in different intracellular components peaks of immobilized fibroblast cells based on SERS Raman spectra obtained from 24 and 48 hours samples containing different C-O concentrations INW platforms along with SEM and fluorescence micrograph. 
The relative change in the peaks of these intracellular biomolecular components over the 6 hours interval for the live fibroblast cells might shed light on probable growth/proliferation behavior of fibroblast cell during incubation period. For example, if the amide III (proteins) peak (Raman shift at $1235 \mathrm{~cm}^{-1}$ ) for high $\mathrm{C}-\mathrm{O}$ concentration nanocarbon was compared over the 6 hours time interval, it was revealed that after 6 hours of incubation there was no such peak. However, this peak appeared with some variation (1232-1240 $\left.\mathrm{cm}^{-1}\right)$ afterwards and became prominent after 24 hours (Figure 4-8). The Raman spectral profile for both 24 and 48 hours for fibroblasts cells fixed on the nanostructures might indicate the presence of healthy cells on the nanostructure and after 48 hours the peaks showed moderately stronger signals (Figure 4-10). The SEM and fluorescence micrograph also confirmed this hypothesis.

The Raman spectral profile for both live and immobilized HeLa cells depicted similar tendency as of fibroblast cell with some unique peaks. However, considering the similarity of profile it might be hypothesized that after 24-hour of culture, there was healthy HeLa cells on the nanostructure. The comparative analysis of the Raman spectra obtained for HeLa cells fixed on the nanostructures of varying C-O concentrations nanocarbon platform after 24 and 48 -hour culture depicted a huge difference in peak appearance. The peaks related to different intracellular biochemical components such as protein, DNA and lipid that appeared in the low $\left(300-1000 \mathrm{~cm}^{-1}\right)$ and medium (1000-2000 $\mathrm{cm}^{-1}$ ) Raman shift range were disappeared in the 48-hour Raman spectral profile. Based on such change it might be hypothesized that there was a change in growth of the adhered HeLa cell and they might show nonproliferating behavior. This hypothesis was supported by the SEM micrograph of HeLa cells after 48 hours of culture (Figure 4-10). 


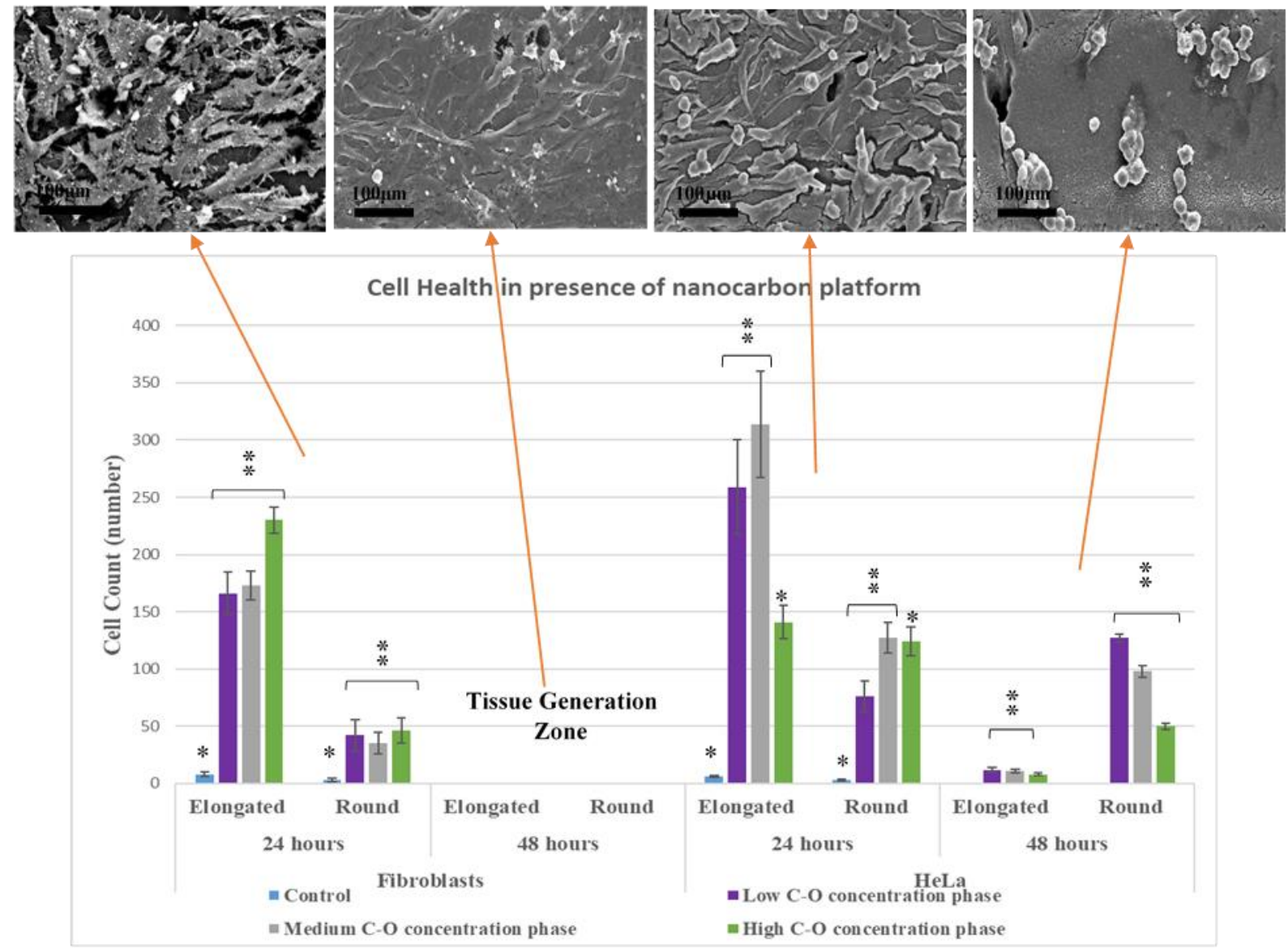

Figure 4-11 Quantitative analysis of different types of fibroblast and HeLa cells on nanocarbon platform with varying $\mathrm{C}-\mathrm{O}$ concentrations. The error bars show the standard error of the mean; experiments were repeated with $\mathrm{n}=3$. Statistical significance is shown with $* \mathrm{p}<0.05$ and $* * \mathrm{p}<$ 0.01 .

To support the Raman spectral profile measurement, a comparative quantitative study of the divergent shape of the fibroblasts and HeLa cells on the nanocarbon platform (Figure 4-11) was carried out. A well-spread elongated polygonal cell might be indicative of the healthy cells, whereas a non-flattened round cell shape revealed non proliferating/apoptotic cells ${ }^{[4-68,4-69]}$. Both the elongated and round cells that adhered on the nanocarbon platform of varying C-O concentrations were counted to identify the cell health in presence of nanocarbon structure. From the graph in Figure 4-9, the number of adhered elongated fibroblasts with a gradual positive 
increment for varying $\mathrm{C}-\mathrm{O}$ concentrations nanocarbon indicated healthy cells on the platform. The further growth of fibroblast cells and the formation of tissue like structure after 48 hours limited the possibility of individual counting of fibroblasts. In contrast, the number of elongated HeLa cells in comparison to the corresponding number of round cells adhered on the different $\mathrm{C}-\mathrm{O}$ concentrations nanocarbon indicated healthy attachment and growth of HeLa cells. After 48 hours of culture, there was a huge increment of round nonproliferating cells in comparison to minimal number of elongated cell which indicated nonproliferating scenario of the attached HeLa cells. These results commensurate with the Raman spectra profile obtained for both fibroblast and HeLa cells.

\subsubsection{SERS Raman spectra of the single fibroblast and Hela cell (immobilized)}

A further analysis was performed to identify the effect of the INW clusters in achieving SERS Raman spectra in individual cell level. The SERS Raman spectra of fibroblast and HeLa cells at the single cell level that adhered on the nanostructure or on the plain graphite substrate outside the nanostructure zone (termed as native) was collected through Renishaw confocal Raman microscope which allows spatially resolved spectroscopy to be carried out in a confocal manner at high speed. In comparison to native cell spectra the spectral profile that obtained from high C-O concentration nanocarbon was prominent and peaks of intracellular biochemical components peaks were prominent. The HeLa cells showed more prominence for different DNA/RNA, protein and lipid peaks in comparison to fibroblast cells. At the single cell level, the characteristic peaks of INW platform (D, G and G' bands) showed the highest intensity. 

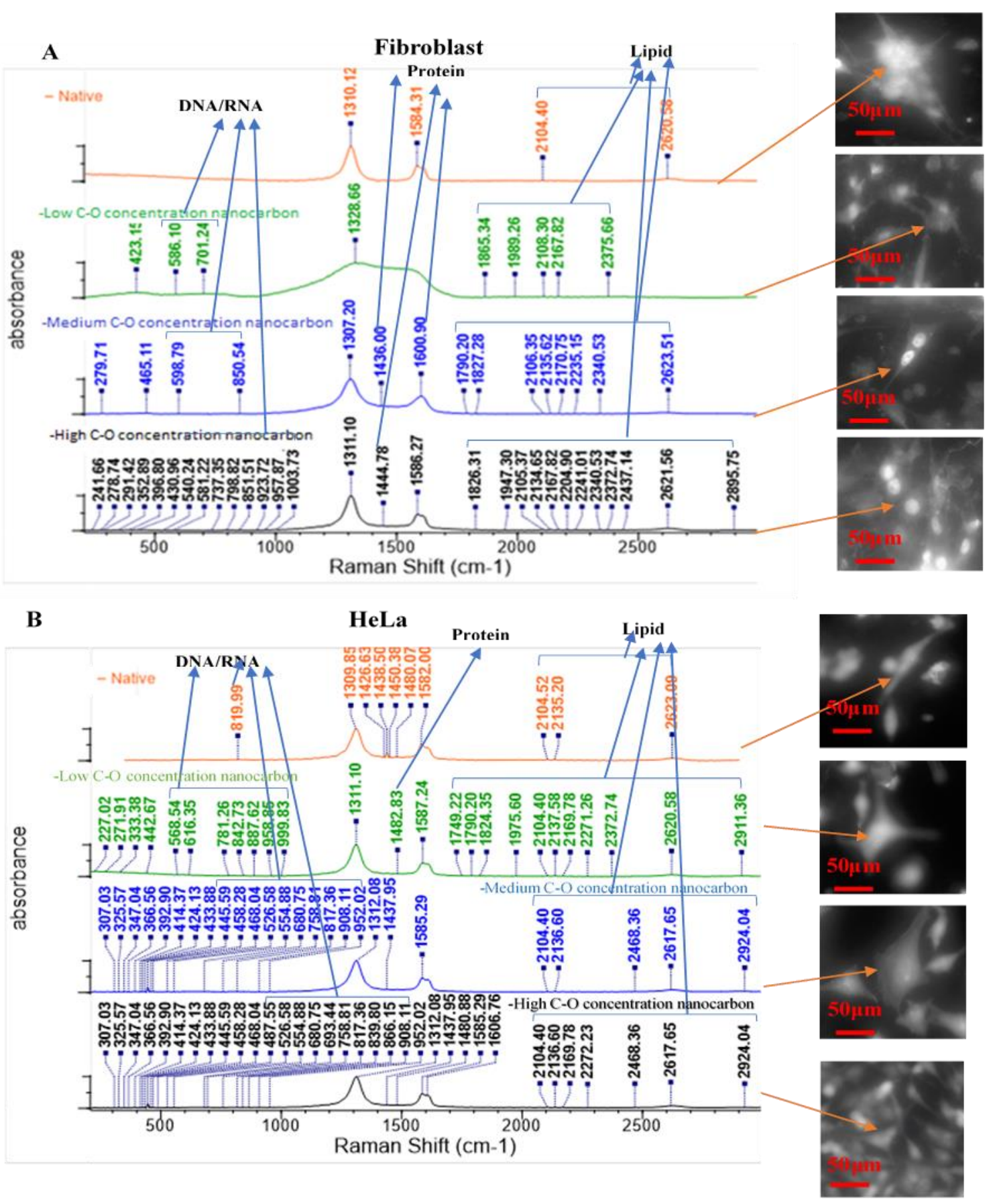

Figure 4-12 (A)SERS Raman spectra of immobilized single fibroblast cell along with confocal image for 24-hour sample containing different C-O concentrations nanocarbon platforms and (B) SERS Raman spectra of immobilized single HeLa cell along with confocal image for 24-hour sample containing different C-O concentrations nanocarbon platforms. 
However, for both fibroblast and HeLa with the increment of C-O concentrations in the INW structure and the decrease in nanocarbon width distribution more peak prominence was observed. The peaks that were appeared here for the single cell level for both the cells were in the same region of those peaks earlier appeared for live cells as well as immobilized cells and based upon which the tentative Raman peak assignment made in Table S1 and Table S2 for fibroblast and HeLa cells. These peaks also appeared due to the presence of intracellular biochemical components like protein, DNA/RNA, and lipid etc.

\subsection{Discussions}

\subsubsection{D interconnected nanocarbon web formation}

Raman spectroscopic approach along with nanostructure/nanoparticle based SERS platform allows the study of the chemical composition of complex bio-sample like cells and shed light on their molecular makeup in health and disease ${ }^{[4-57]}$. Fine tuning of morphology as well as nanochemistry of nanofabricated SERS-active substrate enables sensitive detections of cells ${ }^{[4-70]}$. In this study, the bio-sensitive INW platform on the graphite substrate was achieved with the aid of ultrashort femtosecond laser processing in an ambient atmosphere. The ablation of the graphite surface was attained through mechanical fragmentation, homogeneous nucleation and vaporization when it was irradiated using femtosecond laser pulses ${ }^{[4-71]}$. The multiphoton ionization create a reactive high-temperature plasma plume which contains a combination of neutral carbon (C), carbon radicals $\left(\mathrm{C}_{2}, \mathrm{C}_{3}\right)$ and carbon ions $\left(\mathrm{C}^{+}, \mathrm{C}^{2+}\right)^{[4-41]}$. At the bottom of this expanding plasma plume the heavier radicals and the neutral carbon reside whereas carbon ions lie on the upper part of the plume. The component mixture of the plume varies with the change in ionization energy and creates impact on the morphology variation and the change in nano-chemistry of the created 3D INW platform. When these carbon nano species of the plume condensed and aggregated shaped 
into an interwoven nanocarbon structure. The nanocarbon width varies with the change in ionization energy from low to high and average width decreases with the increase in ionization energy. Oxygen atom which is one of the components of the ambient atmospheric gas come across the boundary of the expanding plume and had a chemical contact with the carbon ions resulting in the creation of $\mathrm{C}-\mathrm{O}$ molecular bonds. Therefore, both $\mathrm{C}-\mathrm{C}$ and $\mathrm{C}-\mathrm{O}$ comprise the chemical structure of created nanocarbon as they deposited at the time of condensation process. The nanochemistry in terms of C-O concentration increases with the increase in ionization energy. The FESEM micrograph analysis (Figure 4-2 C) and XPS analysis (Figure 4-2(D-E)) showed the variation in the topography and chemical properties of the created nanocarbon platform.

\subsubsection{SERS active nanocarbon based detection of fibroblast and HeLa}

The SERS based Raman sensing of fibroblast and HeLa cells might be explained from the interaction among the fibroblast, the HeLa cells and the INW clusters, the constructive components of the interwoven 3D INW platform. Upon adhered, uptake mechanism of the fibroblast and HeLa cells allowed the INW clusters to merge with the membrane and pass through it without

encapsulation. Roiter et. al. ${ }^{[4-72]}$ reported that the curvature of lipid bilayers dropped onto smooth nanoparticles between 22 to $200 \mathrm{~nm}$ diameter does not promote membrane break. The nanocarbon width distribution, in this study, varied between 5.27 and $6.67 \mathrm{~nm}$ (Figure 4-3). The sub-10 nanometer structure of the INW platform helped smooth uptake of the INW clusters by the cells. Again, researchers showed that particles of sizes up to $100 \mathrm{~nm}$ could be taken up by active endocytosis ${ }^{[4-73,4-74,4-75]}$.

Upon internalization, the INW clusters were neighboring intracellular components of the cells. C$\mathrm{C}$ bond of the INW clusters induced charge transfer. Strong charge transfer interaction between INW clusters and intracellular biomolecular components like proteins, nucleic acids (DNA/RNA) 
and lipids might include an increase of the electron transition probability and consequently there is an increment in Raman signal. The presence of SERS active INW clusters inside the cells therefore helped Raman spectroscopic signal enhancement of intracellular biochemical components of cells and hence detection of fibroblasts and HeLa cells.

\subsubsection{Cell health}

Both Raman spectral profile and quantitative analysis of dissimilar cell shape that adhered on INW platform of varying $\mathrm{C}-\mathrm{O}$ concentrations suggested cell specific time dependent opposite nature cell health. For fibroblast, the tissue like structure is an indication of positive/no impact of INW cluster on fibroblast over time. Conversely, the INW cluster had affected negatively on HeLa cells with the passage of time and resulted in the nonproliferating round shape apoptotic cells. These results are in agreement with our previous study ${ }^{[4-76]}$. The presence of the $\mathrm{C}-\mathrm{O}$ chemical bond in the nanocarbon clusters that was internalized into the cell body through endocytic uptake might contribute in positive response of both fibroblasts and HeLa cells after 24-hour culture and helped cell survival, growth and proliferation. These facts were supported by the recent research on cell survival and proliferation in an oxygenated environment ${ }^{[4-77,4-78]}$. Again, the nanocarbon clusters presence inside the cytoplasm might create de-regulation of adhesion-associated genes and corresponding adhesive proteins and affect the energy metabolism of the HeLa cells over the passage of time ${ }^{[4-76]}$. With elapses of time nanocarbon clusters could produce cytotoxicity and might create stress to the HeLa cells ${ }^{[4-79]}$. All these facts together result in cell rounding i.e. apoptotic cell structure. 


\subsection{Summary}

There is an exponential growth in the development of noninvasive label free analytical techniques for studying cells in-vitro. Surface enhanced Raman scattering technique has gained researchers attention as it provides an intrinsic contrast mechanism for identifying components in cells. So far, pure noble metal (gold, silver) based SERS substrate or noble metal assisted carbon nano-platform were used for limited scale bio-sensing of cells and detection of bio molecules only. Carbon nanomatrials/nanostructures were conjugated with noble particles due to their low/no SERS ability and toxicity. However, the carbon nanomaterials were not explored individually so far as nonplasmonic SERS active platform for in vitro cancer/normal cells detection. In this paper, we have reported creation of a novel SERS active non-plasmonic bio-sensing platform based on selffunctionalized biocompatible 3D interconnected nanocarbon web structure for effective detection of HeLa and fibroblast cells along with a guidance regarding cell health. Sub-10 nanometer structure of the INW platform formed the self-internalization capacity and were uptaken by the live cells during incubation. Label-free non-plasmonic SERS functionality attribute of INW platform allowed SERS based Raman sensing of live cells. There was a significant achievement

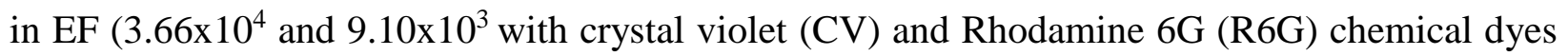
respectively) for the INW platform in comparison to Raman enhancement factors on the surface of monolayer graphene (2 to 17). The SERS based Raman spectra of the time-based Raman spectroscopic monitoring of both live HeLa and fibroblast cells revealed chemical fingerprints of intracellular components like DNA/RNA, protein and lipids. A guideline has been introduced to specify each cell based on the spectroscopic differences of DNA/RNA and protein peaks. The highest Raman signal enhancement achieved for intracellular components for fibroblasts was 4fold and for HeLa was more than 5-fold. Additionally, a procedure for identification of cell health 
might be possible based on the spectral difference of the SERS based Raman spectra of the immobilized cell after 24 and 48 hours. The creation of the self-assembled 3D INW platform containing $\mathrm{C}-\mathrm{O}$ and $\mathrm{C}-\mathrm{C}$ bond mixture which contain all the mentioned unique attributes was done through employment of one step femtosecond laser processing on the non-bio responsive C-C bond containing a graphite plate in ambient conditions. Both morphology and physicochemical properties of created 3D INW platform varies with the change in ionization energy. Nanocarbon width shows gradual reduction with the increment of ionization energy. Mixture of C-O and C-C bonds comprise nanochemistry of the created nanostructure platform and C-O concentration variation was achieved by controlling the ionization energy of the incident laser beam.

This in-vitro study recognized the distinct functionality of self-functionalized 3D INW platform as non-plasmonic SERS active bio-sensing nanoprobe for identification of both HeLa and Fibroblast cells through chemical fingerprints of intracellular components like DNA/RNA, protein and lipids without the need of conjugation of any noble metals. Overall, the 3D INW structure can be used as a viable and versatile SERS active sensing platform for probing intracellular compounds of HeLa and fibroblasts cells and thereby detection of each cell separately and at the same time, shed light on the health of cells. 


\section{Chapter 5}

\section{SERS active Nitrogen-Enriched 3-D Carbon Nanoprobe for Cancerous HeLa Cells Diagnosis.}

\section{Submitted for Review in Nanoscale, March 2018}

Carbonaceous nanostructures based surface-enhanced Raman spectroscopy (SERS) substrates introduce a new edge in the field of SERS despite their individual application as SERS substrates is impeded by their low SERS enhancement and inferior detection sensitivity. Here, we introduce a nitrogen incorporation-assisted approach to create a new nitrogen enriched 3D carbon nanoprobe (N-CNP) sensor platform and demonstrate a label free non-plasmonic SERS based unique technique of early detection of HeLa cancer cells by intracellular sensing. The N-CNP sensor platform yields an enhancement factor of $2.10 \times 10^{4}$ with the crystal violet dye which is in the higher side in comparison to Raman enhancement factors of monolayer graphene (2 to 17). The N-CNP sensor platform demonstrated favorable microenvironment for cell adhesion and SERS hot spots were created inside the HeLa and fibroblast cells through internalization of N-CNP units having physical morphology below ten nanometer nanoprobe width and thereby achieved SERS based live cells Raman sensing. In situ in vitro Raman spectroscopic monitoring of live HeLa and fibroblast cells suggested that the SERS functionality of the N-CNPs not only reveals the chemical fingerprint of the intracellular components (protein, DNA, RNA etc.) within a cell but also guides early detection of cancerous HeLa cells. The highest Raman signal enhancement achieved for intracellular molecules, like DNA, was almost 6-fold in comparison to carbon sensor spectra. The 
femtosecond laser induced self-assembly process resulted in SERS active N-CNP sensor platform for HeLa cell (cancer) diagnostics.

\subsection{Introduction}

Cancer, a leading cause of death worldwide, is defined as an uncontrolled division of abnormal cells in a part of the body. Cervical cancer is the fourth most common cancer in women and the mortality associated to it can be reduced if this disease is detected at the early stage ${ }^{[5-1,5-2,5-3]}$. Since early detection plays a key role in boosting the survival of cancer, the development of a sensitive technique for early discovery is one of the major focuses of cancer research. Therefore, recent efforts have been focused on the development and testing of new conceptual or technological methods for early diagnosis of the disease. Raman spectroscopy enhanced with nanomaterials/nanostructures platform gained much attention due to its promising in terms of implementation and efficiency. Raman spectroscopy, one of the current diagnostic techniques, is progressively explored for early cancer diagnosis ${ }^{[5-4]}$. Molecular changes in cells, tissues or biofluids can be detected and quantified by Raman spectroscopy. The medical diagnostic applications of Raman spectroscopy have recently escalated due to the ability to use advanced optical technologies in the visible or near-infrared spectral range, easy sample preparation and high chemical specificity ${ }^{[5-5,5-6,5-7]}$.

The Raman spectra of cells and tissues that contain many biochemical components, including DNA, RNA, proteins, lipids, and carbohydrates, are a superposition of the contributions from each individual biochemical component. However, Raman spectroscopy often requires high power lasers due to its low scattering efficiency ${ }^{[5-8]}$ and that may cause damage to biological samples. Thus, surface enhanced Raman scattering (SERS) technique utilizing nanostructured surfaces ${ }^{55-9,}$ ${ }^{5-10]}$ improve the sensitivity of traditional Raman spectroscopy while reducing the laser power and 
collection time $^{[5-11]}$. With single molecular sensitivity, SERS can be widely applied to chemical molecule/ion detection, clinical discrimination of cancer tissues etc. ${ }^{[5-12,5-13]}$.

Recently, different researchers used carbon based nanostructures/nanomaterials for diagnostics and bio-sensing ${ }^{[5-14,5-15]}$. Carbonaceous nanostructures due to their different anisotropies and surface properties are good candidate to act as a bio-sensor. Pristine carbon nanotubes (CNTs) contain cytotoxicity due to its synthesis procedure ${ }^{[5-16]}$ and require functionalization to gaining suitability for bio-sensing ${ }^{[5-17]}$. Although CNTs are SERS active, capability CNTs alone is not always sufficient to provide adequate detection of cancer cells due to poor Raman enhancement ${ }^{[5-}$ ${ }^{18]}$. With noble metal (Au, Ag) nanoparticle conjugation, CNTs achieved detection, differentiation and therapy of cancer cells ${ }^{[5-19]}$. In line with CNTs, only 2 to 17-fold Raman enhancement was observed on graphene film ${ }^{[5-20]}$. Therefore, graphene was conjugated with gold nanoparticles and differentiation of human breast normal, cancer and cancer stem cells was achieved ${ }^{[5-9]}$. Graphene quantum dots, another branch of carbonaceous nanomaterials, showed higher Raman enhancement than conventional graphene sheet ${ }^{[5-21]}$. However, it is not used alone as SERS sensor for in-vitro live cancer cell detection. Individual use of carbonaceous nanoparticles as SERS substrate requires high power laser irradiation and long acquisition times to achieve a significant spectrum recording. This process can cause heat damage in cells and tissues. Therefore, the application of individual carbonaceous nanoparticles in Raman spectroscopy-related techniques for biomedical applications is limited.

Thus far, the researchers used carbon nanomaterials as a component of hybridized bio-sensing platform along with noble metal nanoparticles (Ag and $\mathrm{Au}$ ) and attained SERS active detection of various normal and cancer cells. Overall, Carbonaceous nanostructures based surface-enhanced Raman spectroscopy (SERS) substrates introduce a new edge in the field of SERS despite that 
their individual application as SERS substrates is impeded by their low SERS enhancement and inferior detection sensitivity. There is a need for biocompatible carbon nanomaterials/nanostructures that can be applied individually as a label free SERS based platform for in-vitro detection and differentiation of cancer cells.

Herein, we demonstrate a nitrogen incorporation-assisted approach to create a new nitrogen enriched 3D carbon nanoprobe (N-CNP) sensor platform that lead to significant increase in SERS enhancement, probably due to the enhanced charge-transfer resonance ${ }^{[5-17]}$ as well as exciton resonance arising from the judicious control of nitrogen admission in N-CNPs sensor platform. The N-CNPs sensor platform yields reproducible SERS signals with an enhancement factor of $2.10 \times 10^{4}$ with the crystal violet $(\mathrm{CV})$ dye , which is in the higher side in comparison to Raman enhancement factors on the surface of monolayer graphene (2 to 17$)$ obtained in other literature ${ }^{[5-}$ 20]. The created platform showed enhanced microenvironment favorability in terms of both fibroblast and HeLa cell attraction and subsequent adhesion which ultimately reflects its biocompatibility. The microenvironment favorability showed increasing trend with increment in nitrogen enrichment on N-CNPs sensor platform. With the aid of the N-CNPs sensor platform, in the present research, we introduce a label free non-plasmonic SERS based unique technique for potential point-of-care screening and early detection of HeLa cancer cells by intracellular sensing. SERS hot spots were created inside the HeLa and fibroblast cells through internalization of NCNPs unit structures smaller than ten nanometers and thereby achieved SERS based Raman sensing of live cells. The Raman spectroscopic monitoring of live HeLa and fibroblast cells seeded on N-CNP containing platform was done during the incubation period of 24 hours at an interval of 6 hours with handheld nano Raman system. The results depicted peaks related to intracellular biochemical components like proteins, DNA and RNA. The highest Raman signal enhancement 
achieved for intracellular molecules such as DNA was almost 6-fold in comparison to carbon sensor spectra. Dominance of specific peaks in the Raman spectra profile and the significant relative intensity changes for fibroblast and HeLa for live condition suggested a guideline in detecting and differentiating the HeLa cells in comparison to fibroblast and thereby early diagnosis of Hela cells. Femtosecond laser induced self-assembly process results in variation of nitrogen enrichment in the created 3D carbon nanoprobe and exhibited enhanced SERS activity. The simplicity and versatility of the proposed SERS based N-CNPs sensor platform aided Raman detection technique for HeLa (cancer) in comparison to fibroblast (mammalian) cell can be used for cancer diagnostics and/or as a tool for evaluating new cancer therapy.

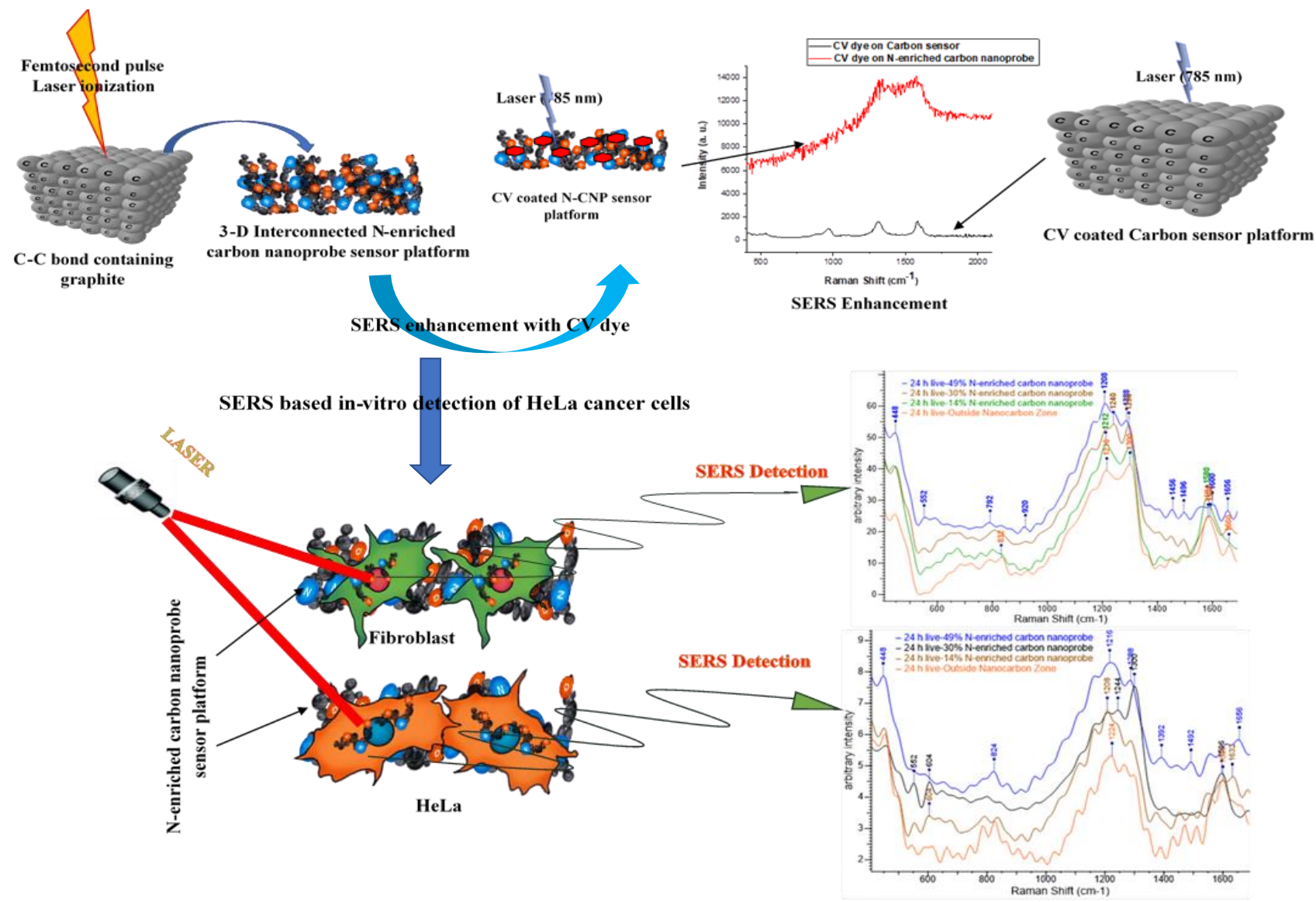


Figure 5.1: Graphical abstract showing formation of 3D interconnected Nitrogen enriched carbon nanoprobe sensor platform, SERS activation with CV dye and subsequent SERS based early detection of HeLa and Fibroblast cells

\subsection{Materials and Method}

\subsubsection{Nitrogen-enriched 3D carbon nanoprobe sensor fabrication}

The generation of the N-enriched 3D carbon nanoprobe (N-CNP) sensor platform was achieved through ionization of an isomolded, very fine grain, high strength graphite plate (3 $\mathrm{mm}$ thickness) (Graphtek LLC, USA) using a Clark-MXR IMPULSE pulsed Yb-doped fibre amplified femtosecond laser system. To prepare the graphite plate ready for ionization processing, each 4 $\mathrm{cm}^{2}$ square plate was polished with sand papers (3M Canada, 1000 grit, 2000 grit and 3000 grit), ultrasonically cleaned (Cole-Parmer 8890 ultrasonic cleaner) with acetone and ethanol and then dried. The consistency of the ultrafast pulsed laser formation mechanism was maintained with the laser wavelength $(1030 \mathrm{~nm})$, the average laser power (15W), the laser pulse width (214fs), the laser beam scanning speed $(1 \mathrm{~mm} / \mathrm{s})$ and irradiation focal spot area $\left(84.62 \times 10^{-8} \mathrm{~cm}^{2}\right)$. An array of lines with varying space designed by EzCAD software was machined on the graphite sample substrate mounted on a fixed stage with computer controlled high precision 2-D translation scanner guided the incident laser beam. The nano topography and chemistry of the created N-CNPs sensor platform was altered with the variation of ultrashort pulse laser fluence (the pulse energy transferred to the substrate per unit area) to $4.43,2.22$ and $0.68 \mathrm{~J} / \mathrm{cm}^{2}$ (referred to as high, medium and low fluence). To facilitate the interaction of the carbon ion with the gaseous species of $\mathrm{N}_{2}$ towards formation of the $\mathrm{N}$-enriched 3D carbon nanoprobe, gaseous species of $\mathrm{N}_{2}$ was introduced into the ion-plume formed by the pulsed laser. The $\mathrm{N}_{2}$ gas was injected at a flow rate of $0.2 \mathrm{MPa}$ 
into the laser-sample substrate interaction zone through nozzles which evenly surround the ionization area.

\subsubsection{Morphological characterization}

The surface morphology of the self-assembled N-CNPs sensor platform was evaluated initially with field emission scanning electron microscope (FE SEM) (Hitachi S-5200). Following SEM, EDX was carried out to determine the elemental composition of irradiated N-CNPs sensor platform. The physical morphology of the resulting N-CNPs sensor platform was imaged with a Leo 912 transmission electron microscope (TEM) on copper mesh grids. The size distribution of created nanostructure was determined through the analysis of the images using Image $\mathbf{J}$ (image processing software).

\subsubsection{Physicochemical characterization}

X-ray photoelectron spectroscopy (XPS) was employed to identify the physicochemical properties of the fabricated N-CNPs sensor platform. XPS analysis deduced the chemical composition of the nanostructures. The XPS data was collected with a Thermo Fisher K-Alpha XPS system using a monochromated Al Ka X-ray source with a 2:1 ellipse spot size of $400 \mathrm{~mm}$ as the major axis. 1 $\mathrm{cm}^{2}$ samples were irradiated with the laser system and used for XPS analysis. To give better energy resolution and peak shape definition regional scans were performed at $50 \mathrm{eV}$ pass energy and 0.1 $\mathrm{eV}$ point spacing. Elemental quantification of the created nanocarbon structure was performed with the Avantage software.

B\&W Tek, Inc NanoRam® handheld Raman system (wavelength $785 \mathrm{~nm}$ at a power of $350 \mathrm{~m} \mathrm{~W}$ ) was engaged for the SERS analysis of the N-CNPs sensor platform with Crystal Violet dye and the SERS enhancement factor was determined. The CV dye due to its large Raman cross-section is popular for SERS analysis. The CV dye was coated onto individual nanostructure areas at 
concentration of $1 \times 10^{-6} \mathrm{M}$ prior to Raman analysis. Each resultant Raman spectra was obtained at 10 s collection time and repeated in triplicate then averaged.

\subsubsection{Cell culture}

Both human cervical cancer cells (HeLa, ATCC, American Type Culture Collection, ATCC No. CCL-2) and Fibroblasts (NIH-3T3, ATCC, USA) were employed in cell experiments to ascertain the differences of mammalian and cancer cell lines in response to their biochemical fingerprints. These cells were grown in Dulbecco's modified Eagle's medium (DMEM)/F12 supplemented with $10 \%$ fetal bovine serum (FBS) and $1 \%$ penicillin/streptomycin, and the cultures were incubated at $37{ }^{\circ} \mathrm{C}$ in a humidified $5 \% \mathrm{CO}_{2}-95 \%$ air atmosphere.

\subsubsection{Fibroblast and HeLa Cells seeding on N-CNPs sensor Platform}

Prior to addition of cell culture medium, N-CNPs sensor platforms were exposed to UV light for $20 \mathrm{~min}$ for sterilization. Subsequently, the platforms were placed inside Petri dishes containing 3 $\mathrm{ml}$ of DMEM/F12 medium and 10\% FBS per dish, and the cells were seeded at a density of $10^{5}$ cells $/ \mathrm{ml}$. The Petri dishes were placed in an incubator for $24 \mathrm{~h}$. During the incubation period, Raman spectra of the live cells along with the nanostructures were collected at the interval of every 6 hours $(6,12,18$ and 24 hours) using the B\&W Tek, Inc NanoRam® handheld Raman system.

\subsubsection{Fluorescence microscopy of Cells.}

The samples were first fixed in methanol-free paraformaldehyde and then, incubated in skimmed milk to prevent nonspecific binding. Further incubation of the samples was then performed using Alexa Fluor 488 phalloidin (Life Technologies) to stain the actin and the cytoskeleton, followed by DAPI (Life Technologies) to stain the nucleus. The samples were studied using an epifluorescent Nikon E-400 microscope with a fluorescein isothiocyanate (FITC) and DAPI filter. The data were recorded using a DS-5M-U1 color digital camera (Nikon, Canada). 


\subsubsection{Statistics}

Experiments were carried out in triplicate, and the data represented the mean \pm standard deviation, unless otherwise mentioned. The cell counting was carried out using FM images and image processing software. One-way analysis of variance (ANOVA) was used to evaluate the statistical significance, with $* \mathrm{p}<0.05$ and $* * p<0.01$ suggesting significant difference.

\subsection{Results and Discussion}

\subsubsection{N-CNP sensor platform formation}

$\mathrm{N}$-CNP sensor platform was synthesized by femtosecond laser processing of the graphite plate, as depicted in the schematic in Figure 5-2 (A). The laser induced ionization kinetically controlled the self-assembly synthesis of N-CNP sensor platform and introduced unique attributes to its nanostructure. With the precise guidance of a computerized galvano scanner, the $2 \mathrm{D}(\mathrm{X}-\mathrm{Y})$ movement of laser beam transferred a predesigned array of lines onto the surface of the graphite sample. Femtosecond laser processing of the graphite sample, assisted with megahertz pulse repetition rate, caused surface ablation, nanoparticle evaporation and formation of vapor plume. In the dense vapor plume, there are different forms of carbon, namely carbon ions, carbon radicals and neutral carbon ${ }^{[5-22]}$ and air molecules containing oxygen ions. The gaseous species of $\mathrm{N}_{2}$ was introduced in the ion plume through nozzles. The ion plume expanded into the surrounding air and its temperature dissipated and thus process of nucleation occurred and created self-assembled nanostructure containing $\mathrm{C}-\mathrm{C}, \mathrm{C}-\mathrm{N}$ and $\mathrm{C}-\mathrm{O}$ bonds in the plume. Later upon collided and aggregated of these nano-species, $\mathrm{N}$-enriched 3D interconnected carbon nanoprobe was formed. Complex dynamics of plume expansion as well as substrate property and ionization energy influence the nanotopography and the altered nanochemistry of the fabricated structure on the substrate. For this study, we used three different ultrashort pulse laser fluence of 4.43, 2.22 and 
$0.68 \mathrm{~J} / \mathrm{cm}^{2}$ (referred to high, medium and low ionization energy), along with beam scanning speed of $1 \mathrm{~mm} / \mathrm{s}$ to achieve variation in the nano-topography and chemistry of the created nanocarbon structure.

Femtosecond laser pulses irradiated the graphite surface and the ablation thereof attained through mechanical fragmentation, homogeneous nucleation and vaporization ${ }^{[5-23]}$. A reactive hightemperature plasma plume created with the multiphoton ionization contained a combination of neutral carbon $(\mathrm{C})$, carbon radicals $\left(\mathrm{C}_{2}, \mathrm{C}_{3}\right)$ and carbon ions $\left(\mathrm{C}^{+}, \mathrm{C}^{2+}\right)^{[5-22]}$. At the bottom of this expanding plasma plume the heavier radicals and the neutral carbon reside whereas carbon ions lie on the upper part of the plume. The component mixture of the plume varies with the change in ionization energy and creates impact on the morphology variation and the change in nanochemistry of the created 3D N-CNP sensor platform. The width of the carbon nanoprobe varies with the change in ionization energy from low to high and average width decreases with the increase in ionization energy. The gaseous nitrogen ion species supplied through nozzle into the plume and Oxygen atom which is one of the components of the ambient atmospheric gas had a chemical contact with the carbon ions resulting in the creation of $\mathrm{C}-\mathrm{N}$ and $\mathrm{C}-\mathrm{O}$ molecular bonds. Therefore, $\mathrm{C}-\mathrm{C}, \mathrm{C}-\mathrm{N}$ and $\mathrm{C}-\mathrm{O}$ comprise the chemical structure of created carbon nanoprobe as they deposited at the time of condensation process. The nano-chemistry in terms of C-N concentration increases with the increase in ionization energy. 
A

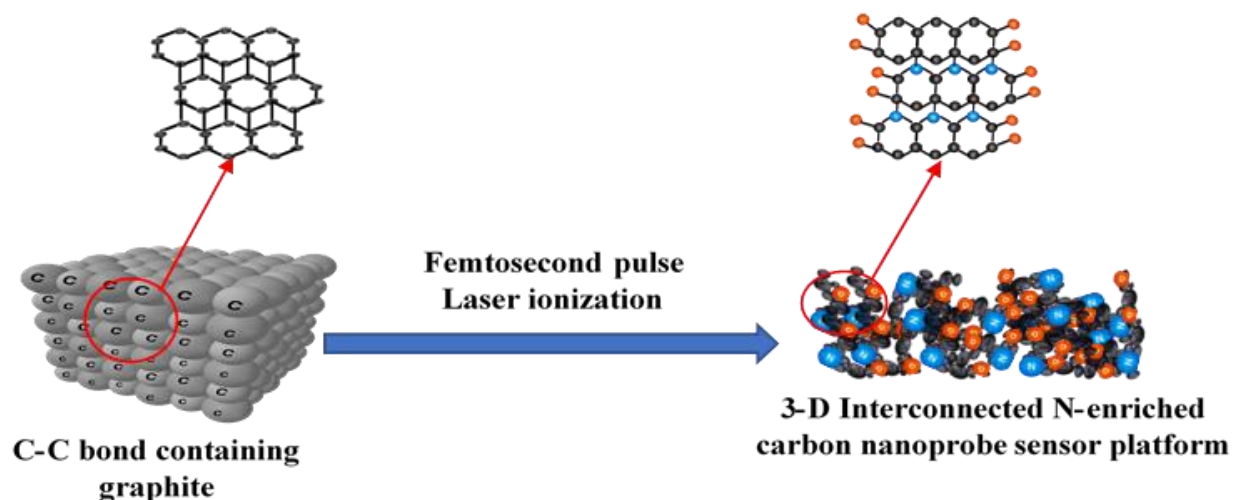

B
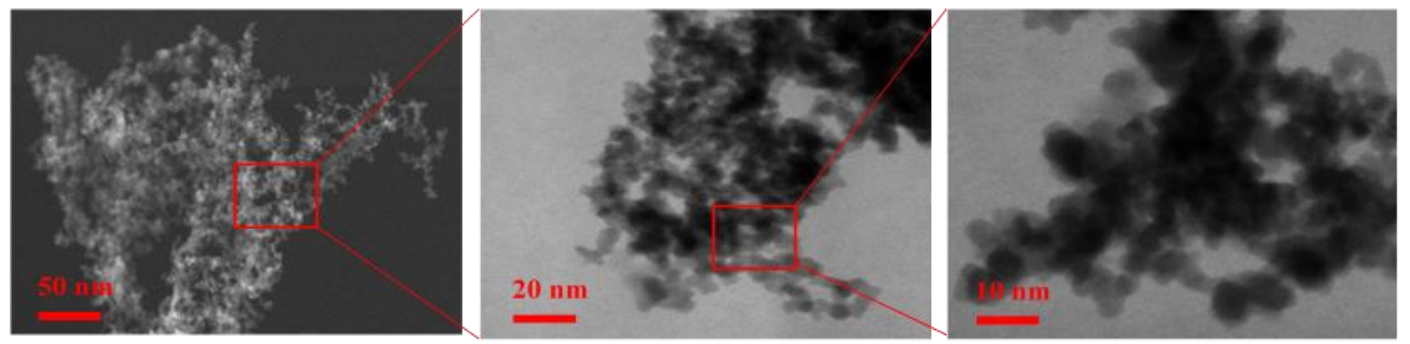

Figure 5-2. A) Schematic illustration depicting the one-step femtosecond laser synthesis of the Nenriched 3D interconnected carbon nanoprobe B) FESEM and TEM images showing physical morphology of the created $\mathrm{N}$-enriched 3D interconnected carbon nanoprobe.

\subsubsection{Morphology and physicochemical characterization of N-CNP sensor platform}

The FE-SEM EDX elemental mapping of the created N-CNP sensor platform (Figure 5-3 A) patterns confirmed that the N-CNP structures mainly consisted of carbon, nitrogen and oxygen. Careful adjustment of laser processing parameters changed the graphite plate surface that contains basically $\mathrm{C}-\mathrm{C}$ bonds and created 3D interconnected nitrogen enriched carbon nanoprobe with $\mathrm{C}$ $\mathrm{C}, \mathrm{C}-\mathrm{N}$ and $\mathrm{C}-\mathrm{O}$ bonds. The N-CNP width describe the physical morphology of the 3D N-CNP sensor platform (Figure 5-3 B). With the increment of ionization energy from low to high, there is a gradual reduction of the N-CNP width. A denser plume generated from higher ionization energy leads to a more compact N-CNP nanostructure. The average size of the carbon nanoprobe width 
varied from 6.10 to $5.40 \mathrm{~nm}$ with the increase in the ionization energy from 0.68 to $4.43 \mathrm{~J} / \mathrm{cm}^{2}$. The frequency histograms of the carbon nanoprobe width distribution (Figure 5-3 B) showed that higher ionization energy tends to have a narrower width distribution. This observation agrees with our previous observation of the femtosecond laser synthesis of the Si nanostructure ${ }^{[5-24]}$. The characteristic XPS spectra of the graphite substrate and the created 3D interconnected N-CNP sensor platform for different $\mathrm{C}-\mathrm{N}$ concentration phases on the graphite substrate along with a quantitative assessment of the major elements are presented in Figure 5-3 C, D. XPS spectra show three characteristic peaks at 285.08, 400.08 and $533.08 \mathrm{eV}$ for the N-CNP sensor platform and the native carbon sensor, which can be ascribed to C 1s, N1s and O 1s, respectively (Figure 5-3 C). Both the reactive gases, such as nitrogen and oxygen, presented during the interaction of lasermater in the surrounding atmosphere. Hence, $\mathrm{C}-\mathrm{N}$ and $\mathrm{C}-\mathrm{O}$ bonds formed as both nitrogen and oxygen took part in the chemical transformation with the carbon ions. Therefore, $\mathrm{C}-\mathrm{C}, \mathrm{C}-\mathrm{N}$ and $\mathrm{C}-\mathrm{O}$ bonds, three major chemical bonds present in the created N-CNP sensor platform. The presence of nitrogen in the N-CNP sensor platform increases with the increase in the ionization energy. 

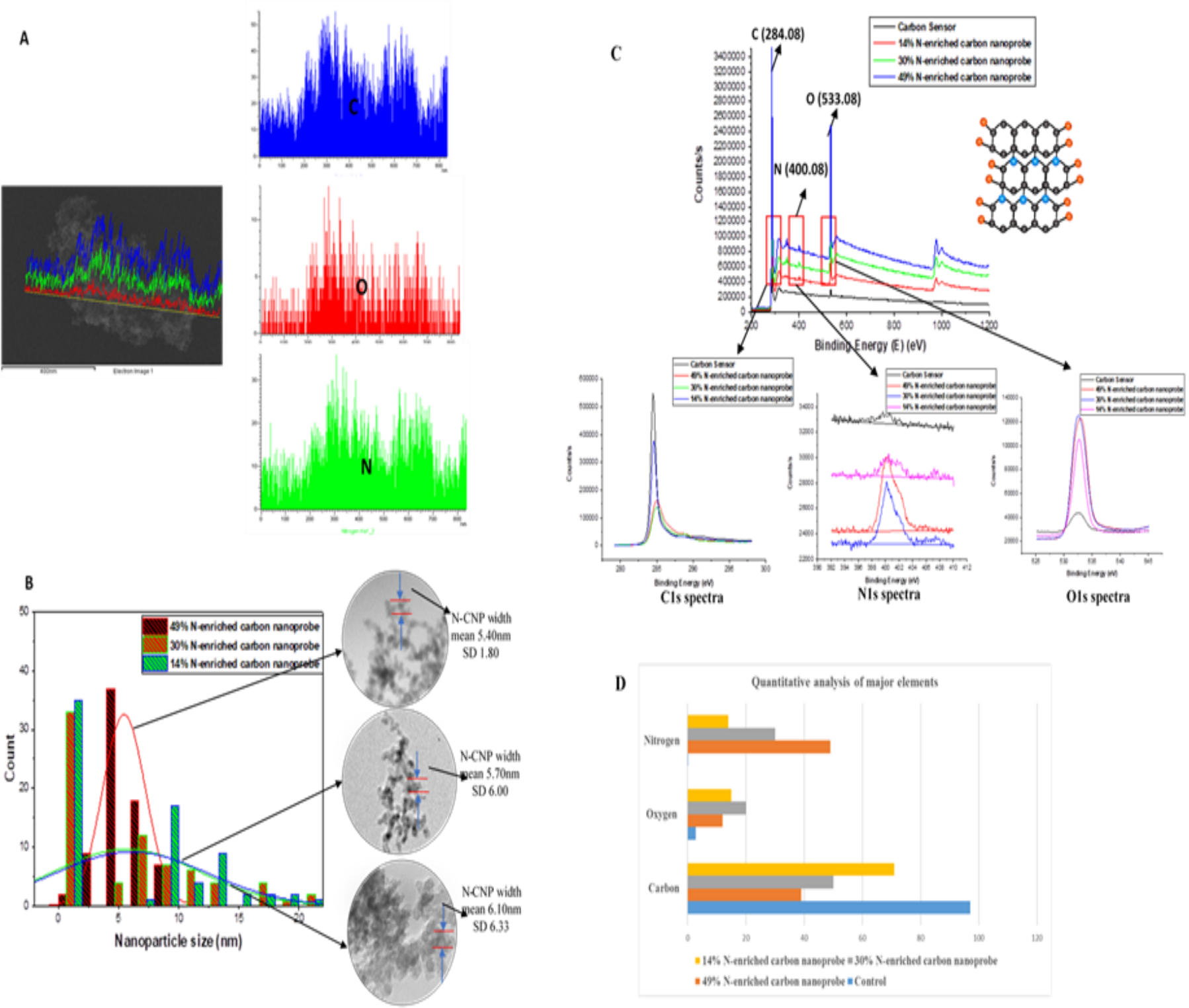

Figure 5-3. (A) FSEM-EDX elemental mapping of the created N-CNPs sensor platform (B) Nenriched carbon nanoprobe width frequency histogram; (C) XPS spectra of the N-CNP structures with different $\mathrm{C}-\mathrm{N}$ percentage phases along with the extended $\mathrm{C} 1 \mathrm{~s}, \mathrm{~N} 1 \mathrm{~s}$ and $\mathrm{O} 1 \mathrm{~s}$ spectra and the (D) corresponding quantitative analysis of the major elements in the created N-CNPs sensor platform.

From the quantitative assessment of the major elements (Figure 5-3 D) the nitrogen percentage are measured as $14 \%, 30 \%$, and $49 \%$ at ionization energies of $0.68,2.22$, and $4.43 \mathrm{~J} / \mathrm{cm}^{2}$, respectively. Therefore, the created different $\mathrm{C}-\mathrm{N}$ concentration bearing $\mathrm{N}-\mathrm{CNP}$ sensor platforms are termed as 
$49 \%, 30 \%$ and $14 \% \mathrm{~N}-\mathrm{CNP}$ sensor platform for this study. The first peak position, which is at $285.08 \mathrm{eV}$ is interpreted as the $\mathrm{sp}^{3}$ hybridized carbon ${ }^{[5-25]}$. The second peak position at $400.08 \mathrm{eV}$ is interpreted as $-\mathrm{C} \equiv \mathrm{N}$ bond of three fold nitrogen ${ }^{[5-26]}$. On the other hand, the peak position at $533.08 \mathrm{eV}$ interpreted as the $\mathrm{C}-\mathrm{O}$ bonds that correspond to the chemisorbed oxygen ${ }^{[5-27]}$.

\subsubsection{SERS efficiency of N-CNP sensor platform and EF calculation}

Crystal violet dye was used to recognize the SERS enhancement factor of the 3D N-CNP sensor platform (Figure 5-4 A). This is a popular dye for SERS analysis due to its large cross section.

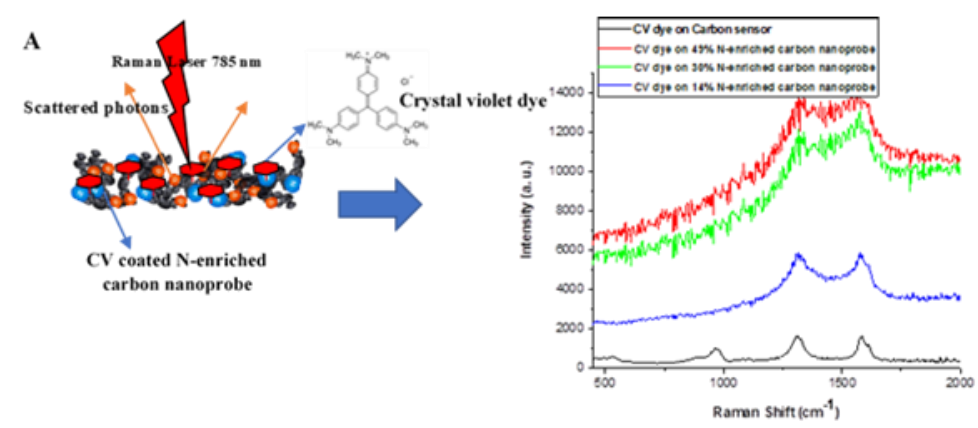

B

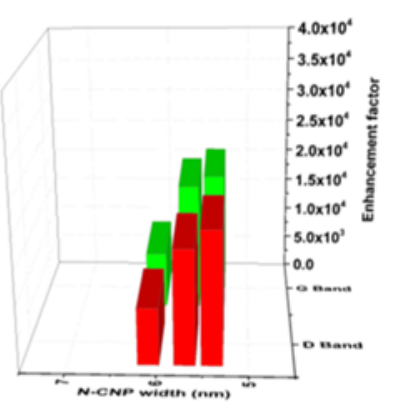

C

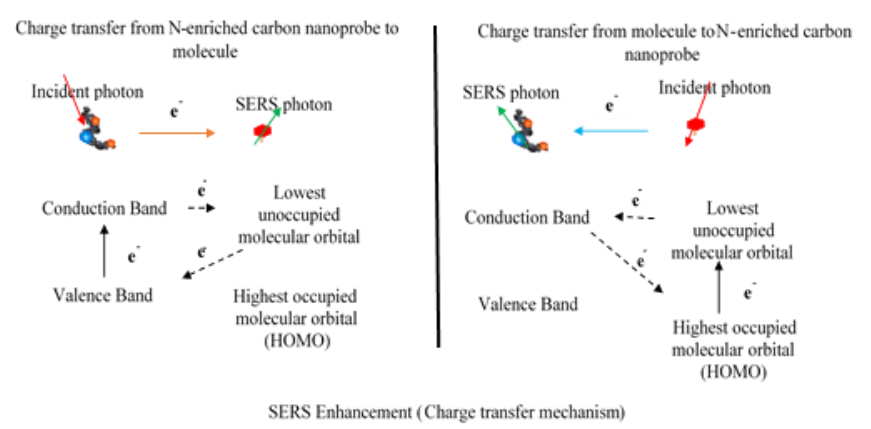

Figure 5-4. (A) SERS Raman enhancement of N-CNP sensor platform with CV dye (B) Raman EF values with CV dye for D and G band at different N-CNP width (C) Schematic of the chargetransfer mechanism principle for SERS enhancement.

The details of the procedure used to determine the enhancement factor (EF) value of the N-CNP sensor platform is described in the Apendix 2. A significant enhancement in the intensity of the characteristic G band and D band Raman peaks of CV dye on the N-CNP structure of varies C-N 
percentage in comparison to that of the bulk carbon sensor was observed, which was evident from both the Raman spectra and EF values (Figure 5-4 A, B). It is detected that the C-C bond containing carbon sensor (the surface layer of the bulk graphite plate having C-C bond) spectra have no or minimum response to the presence of the $\mathrm{CV}$ dye. However, when $\mathrm{CV}$ dye is coated onto the NCNP sensor platform, the characteristic peaks are clear and well defined. We observed a maximum enhancement of $2.10 \times 10^{4}$ for the $\mathrm{CV}$ dye at $10^{-6} \mathrm{M}$ concentration. Highest $\mathrm{EF}$ values were achieved for lower N-CNP width which might be due to higher cross section area associated with lower NCNP width. Both electromagnetic mechanism (EM) and Chemical mechanism (CM), are considered for SERS enhancement phenomena ${ }^{[5-9,5-28]}$. The basis for EM is the enhancement of the local electromagnetic field that results in a significant increase in the Raman scattering cross section. The surface plasmons excited by the incident light is the main contributor to the electromagnetic enhancement ${ }^{[5-29]}$. The enhancement factor for the EM enhancement can reach to $10^{10}$ or more ${ }^{[5-30]}$. In $\mathrm{CM}$ enhancement ${ }^{[5-31]}$, a new resonance state is generated through a charge transfer between the substrate and absorber. Here the cross section of the Raman scattering increases as the positive and negative charge in the molecule become more separated. $\mathrm{CM}$ enhancement offer an enhancement factor of 10 to $10^{2[5-20]}$. In our study, it is presumed that $\mathrm{CM}$ mechanism employed for Raman signal enhancement with an enhancement factor of $10^{4}$ which is on the higher side in comparison to Raman enhancement factors on the surface of monolayer graphene (2 to 17) obtained in other literature ${ }^{[5-20]}$. Therefore, the N-CNP sensor platform is highly SERS active and can detect an analyte much more readily than the carbon sensor.

The SERS efficiency of N-CNP sensor platform might be explained with CM enhancement mechanism. The schematics in Figure 5-4 C explains the principle of the charge-transfer mechanism for $\mathrm{CM}$ enhancement. This mechanism requires charge transfer between the molecule 
and the substrate and the distance between the molecule and the substrate need to be below $0.2 \mathrm{~nm}$ [5-32, 5-33]. The core C-C bond of the N-CNP sensor platform might induce charge transfer which was similar to graphene ${ }^{[5-34]}$. When an analyte molecule is in close proximity to an N-CNP sensor surface and is excited by a photon source the charge-transfer resonance happens ${ }^{[5-35]}$. The valence band (VB) and conduction band (CB) energy levels of the N-CNP structure should be relatively comparable to the lowest unoccupied molecular orbital (LUMO) and the highest occupied molecular orbital (HOMO) of the dye molecule respectively. Electrons can travel along the showed path in the figure thermodynamically from either the N-CNP structure to the molecule or vice versa if these energy levels are within the range of each other and the incident light has enough energy. When a photon excites an electron from the VB to the $\mathrm{CB}$, it is transferred to the LUMO through resonant tunneling because they are neighboring each other and have comparable energy level. This electron transfers energy to a vibrational state of the molecule and causes a SERS photon to be emitted from the molecule. Then it decays back to the CB. Likewise, through resonant tunneling an electron might be excited by a photon from the HOMO energy level to the LUMO energy level and transferred to the CB of the N-CNP structure. After transferring energy to the vibrational state of the molecule the electron will decay to the ground energy level of the molecule and thus causes emission of SERS photon. 


\subsubsection{Favorable micro environment by N-CNP sensor platform}

\subsubsection{Favorable micro environment}

Micro-environment favorability towards attraction and subsequent cell adhesion is important for Raman spectroscopic bio sensing of both HeLa and fibroblast cells. Figure 5-5 (A, B and D) explained the process for identification and quantitative measurement of Micro-environment favorability. Quantitative analysis of the fibroblasts and HeLa cells adhered on the N-CNP sensor platform with different percentage of $\mathrm{C}-\mathrm{N}$ phase (Figure 5-5 A) indicating favorable microenvironment for SERS assessment. 49\% C-N phase containing N-NCP sensor platform showed highest cell adhesion for both fibroblast and HeLa cells. There was gradual decrement as the percentage $\mathrm{C}-\mathrm{N}$ dominant phase decreases. A highest 14 -fold favorability ratio was achieved for $\mathrm{C}-\mathrm{N}$ phase containing N-NCP sensor platform in comparison to the control substrate (figure 5$5 \mathrm{D})$.

A

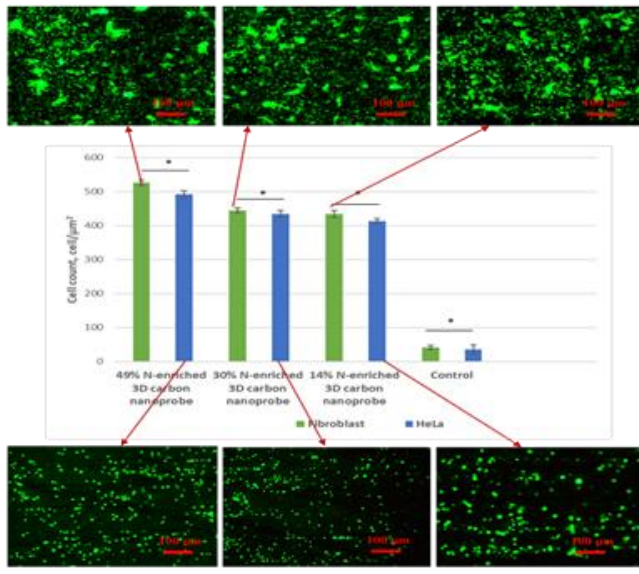

C

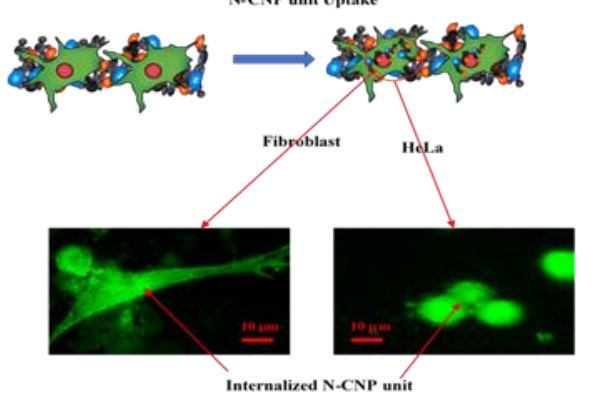

B Micro-environment favorability of N-CNP sensor platform for cell adhesion
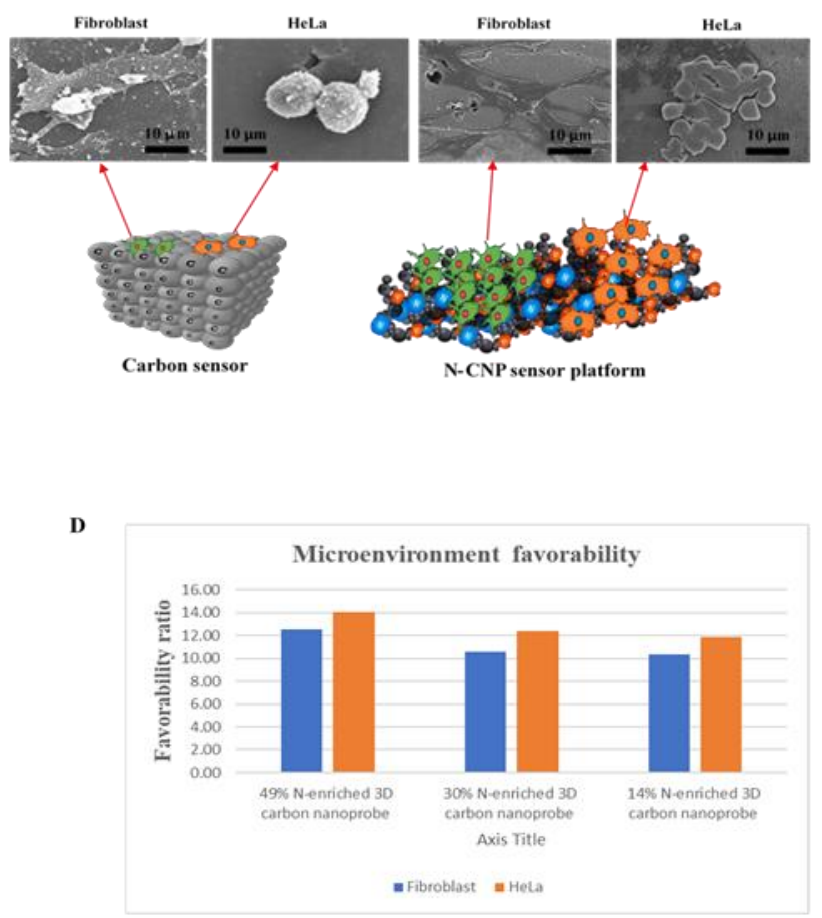
Figure 5-5. (A) Quantitative analysis of the fibroblasts and HeLa cells adhered on the N-CNP sensor platform with different percentage $\mathrm{C}-\mathrm{N}$ dominant phases indicating favorable microenvironment for SERS. The error bars show the standard error of the mean; experiments were repeated with $\mathrm{n}=3$. Statistical significance is shown with $* \mathrm{p}<0.05$ and $* * \mathrm{p}<0.01(\mathrm{~B}) \mathrm{N}-$ CNP sensor platform showed higher microenvironment favorability for cell adhesion (C) Schematics showing N-CNP unit structure uptake by the fibroblast and HeLa cytoskeletons attached to the N-CNP sensor platform and (D) The favorability ratio showed an increasing trend with the increment of C-N enrichment in the N-CNP sensor platform.

Upon seeding, both fibroblast and HeLa cells had an affinity to attach to the areas of higher protein adsorption sites. In comparison to carbon sensor plate the N-CNP sensor platform has a higher protein adsorption area ${ }^{[5-36,5-37,5-38]}$ and hence attracted the cells and primary attachment and subsequent cell adhesion took place.

\subsubsection{N-CNP unit structure uptake}

Local optical fields necessary for intracellular probing is provided through cellular uptake ${ }^{[5-39]}$. NCNP unit structure uptake process by both HeLa and fibroblast cells during the period of incubation was portrayed in figure 5-5 C. The fluorescence micrographs (figure 5-5 C) evidenced the presence of N-CNP unit structure inside the fibroblast and HeLa cells. It might be hypothesized that the internalization of N-CNP unit structure, a constructive components of the interwoven 3D N-CNP sensor platform, was happened through endocytic uptake due to the contiguity of cells to the carbon nanoprobe platform. The endocytic process of taking up N-CNP unit structure by cells was determined by the nanoparticle size, shape, surface charge as well as type of cells ${ }^{[5-40,5-41]}$. Upon adhered, uptake mechanism of the fibroblast and HeLa cells allowed the N-CNP unit structure to merge with the membrane and pass through it without encapsulation. Roiter et. al. ${ }^{5-}$

${ }^{42]}$ mentioned that the curvature of lipid bilayers dropped onto smooth nanoparticles between 22 to $200 \mathrm{~nm}$ diameter does not promote membrane break. The carbon nanoprobe width distribution, 
in this study, varied between 5.40 and $6.10 \mathrm{~nm}$ (Figure 5-3). This said structure of the N-CNP containing platform helped smooth uptake of the N-CNP unit structure by the cells. Again, researchers showed that particles of sizes up to $100 \mathrm{~nm}$ could be taken up by active endocytosis ${ }^{[5-}$ 43, 5-44, 5-45]. The presence of internalized N-CNP unit structure inside fibroblast and HeLa cells (figure 4C) evidenced N-CNP unit structure uptake by the endocytosis process ${ }^{[5-46,5-47]}$.

\subsubsection{SERS Raman spectra of the live Hela and fibroblast cells}

The Raman spectroscopic monitoring of live HeLa and fibroblast cells seeded on N-CNP sensor platform was done during the incubation period of 24 hours at an interval of 6 hours with handheld nano Raman system. Figure 6-6 A, B showed the live Raman spectra of both cell lines. The cells were seeded covering the entire sample which constitutes both N-CNP sensor platform and the native carbon sensors. The Raman spectra collected outside the nanostructure zone have C-C containing surface along with seeded cells.

\subsubsection{SERS spectra of live fibroblast cells}

Suitable microenvironment created by the N-CNPs containing platform attracted the live fibroblast cells toward it and cell adhesion took place. Figure 6-6 A depicted the Raman response of live fibroblast cell on different C-N percentage N-CNP sensor platform for 24 hours incubation period. A decent intracellular component response of fibroblast cells to Raman signal was recorded after 6 hours of incubation. This response was varied over the next every 6 hours-time within total 24 hours incubation period. A tentative assignment of Raman peaks was tabulated in Table S3 in Apendix 5 for live fibroblast cells with reference to the earlier studies ${ }^{48,49}$. Different intracellular molecular fingerprints such as protein $\left(1212 \mathrm{~cm}^{-1}, 1232 \mathrm{~cm}^{-1}, 1472 \mathrm{~cm}^{-1}, 1600 \mathrm{~cm}^{-1}, 1640 \mathrm{~cm}^{-1}\right)$, DNA $\left(800 \mathrm{~cm}^{-1}, 808 \mathrm{~cm}^{-1}, 816 \mathrm{~cm}^{-1}, 1296 \mathrm{~cm}^{-1}\right)$ and RNA $\left(656 \mathrm{~cm}^{-1}, 776 \mathrm{~cm}^{-1}, 780 \mathrm{~cm}^{-1}\right)$ were identified. 


\subsubsection{SERS spectra of live HeLa cells}

Figure 6-6 B depicted the Raman response of live HeLa cell on different C-N percentage N-CNP sensor platform for 24 hours incubation period with 6 hours intervals along with Raman signal enhancement factor variation within the said period. A significant response for HeLa live cell to Raman signal was recorded after 6 hours of incubation in comparison to fibroblast. It was obvious as HeLa showed more affinity toward the N-CNP sensor platform because of higher favorability ratio than fibroblast (figure 6.5D). This response was continued upto 12 hours of incubation. However, both peak intensity and number of peaks appearance reduced next two 6-hour period within the total 24-hour incubation time. A similar tentative assignments of Raman peaks was tabulated in Table S4 (Apendix 5) for live HeLa cells on different C-N concentration N-CNP sensor platform. The identified intracellular molecular fingerprints were DNA $\left(752 \mathrm{~cm}^{-1}, 800 \mathrm{~cm}^{-}\right.$

$\left.{ }^{1}, 804 \mathrm{~cm}^{-1}, 812 \mathrm{~cm}^{-1}, 1288 \mathrm{~cm}^{-1}\right)$, RNA $\left(668 \mathrm{~cm}^{-1}, 764 \mathrm{~cm}^{-1}, 784 \mathrm{~cm}^{-1}, 824 \mathrm{~cm}^{-1}\right)$, proteins (1200 $\left.\mathrm{cm}^{-1}, 1280 \mathrm{~cm}^{-1}, 1596 \mathrm{~cm}^{-1}, 1656 \mathrm{~cm}^{-1}\right)$.

\subsubsection{SERS Raman spectra of the immobilized fibroblast and Hela cells}

An in-depth study of the Raman spectroscopy of the immobilized fibroblasts and HeLa cells that adhered on the N-CNP sensor platform was also carried out and analyzed along with the fluorescence micrograph of the same (Figure 6-6 C, D). The Raman spectra was collected after 24 hours of incubation after fixing the cells on the N-CNP sensor platform. The SERS based Raman spectral fingerprint of DNA, RNA and protein was distinctive for fibroblast rather than HeLa cells. This analysis was in line with the result received earlier in terms of live cell SERS based Raman sensing. 


\subsubsection{SERS effect on live Fibroblast and HeLa cells}

The Raman signal enhancement factor for the intracellular components like DNA, RNA and protein of both fibroblasts and HeLa cells was calculated to identify the effect of N-CNP sensing platform on the Raman spectral quality. DNA showed a highest Raman signal enhancement of almost 6-fold in comparison to native spectra for both the cell lines. The Raman enhancement factor varied over the 24 hours incubation period. However, with the increment of the C-N percentage phase of N-CNP sensor platform there is an increment of Raman enhancement factor.

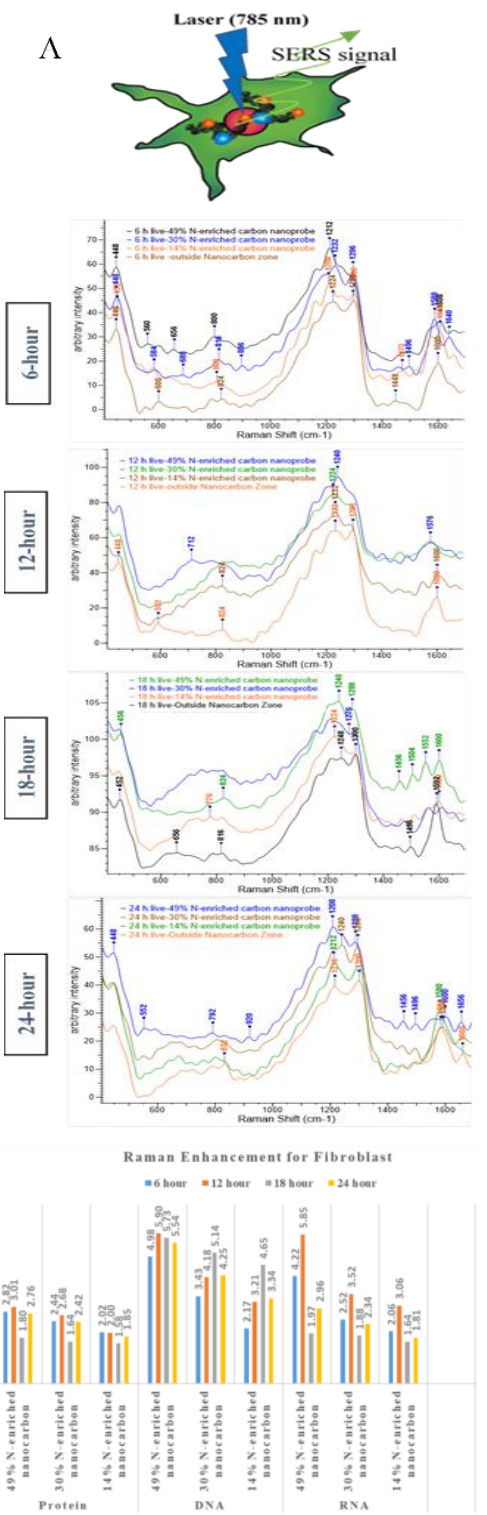

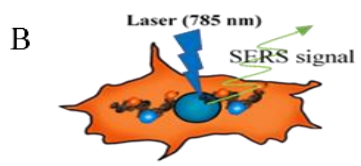
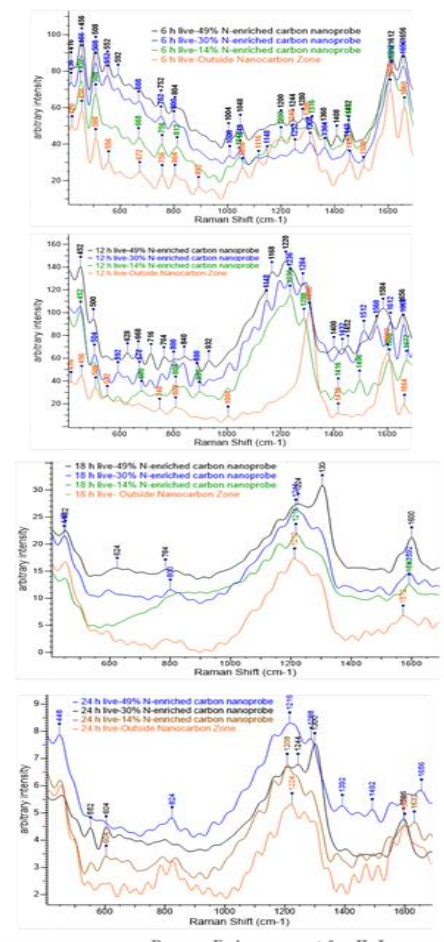

Raman Enhancement for HeLa

-6 6our "12 hour $\quad=18$ hour $=24$ hour

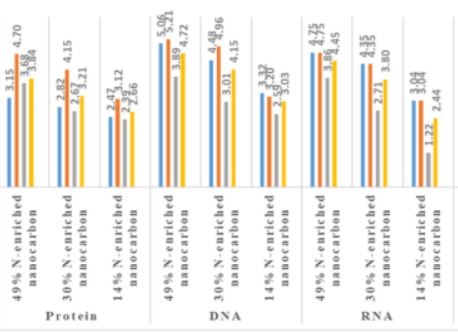

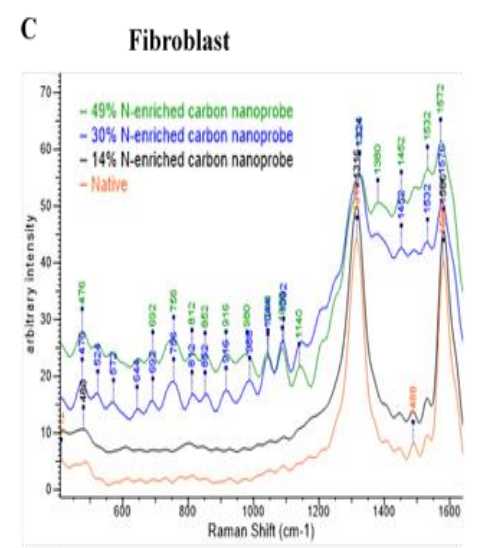
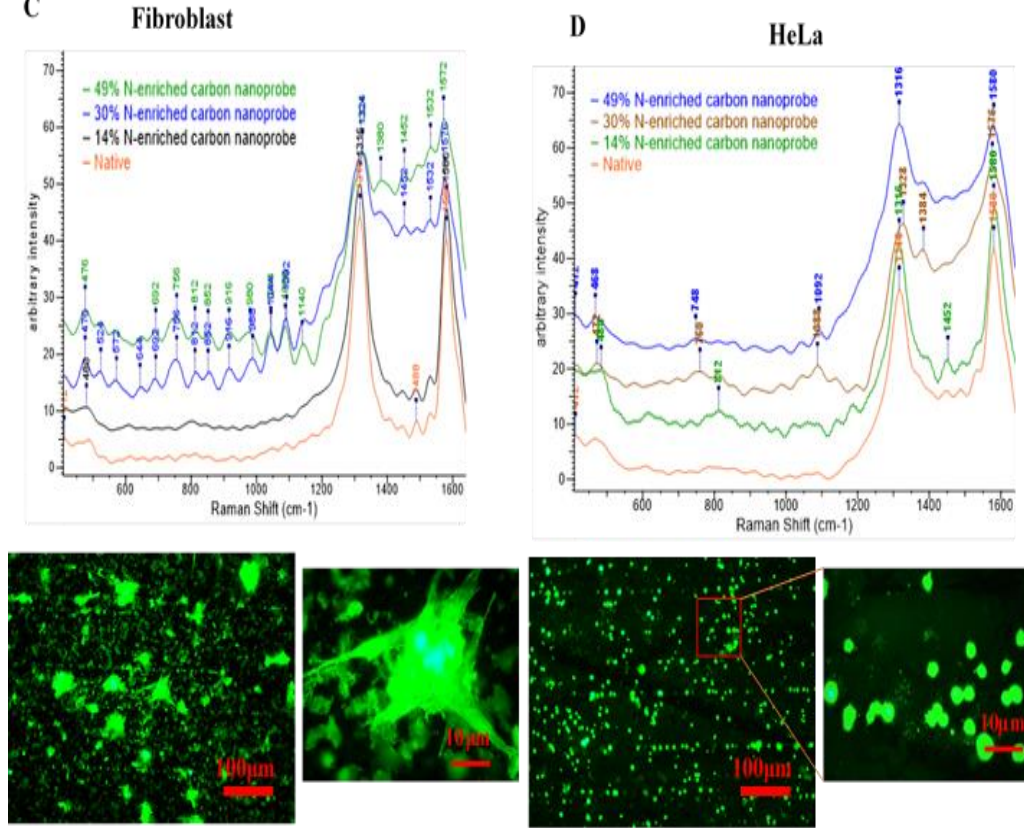

E
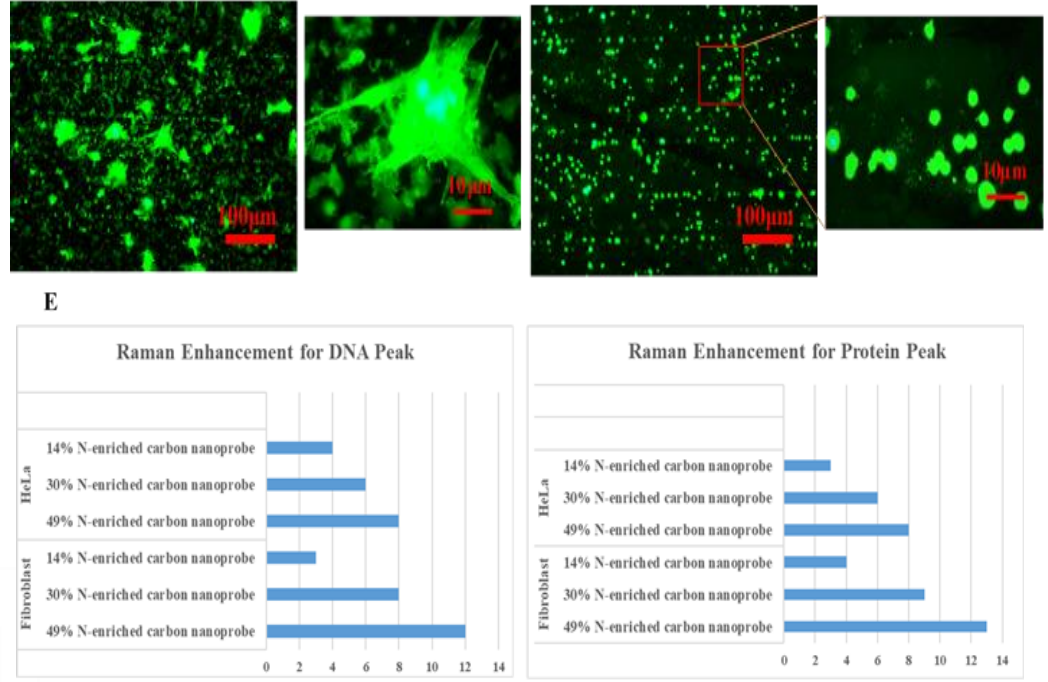

Raman Enhancement for Protein Peak

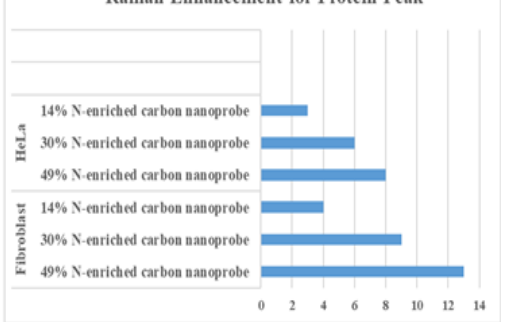


Figure 5-6 SERS Raman spectra of (A) live fibroblast cell and (B)HeLa cells seeded on different C-N percentage N-CNP sensor platform for 24 hours incubation period with 6 hours intervals along with Raman signal enhancement factor variation within the said period $(\mathrm{C})$ Variation in different intracellular components peaks of immobilized fibroblast cells based on SERS Raman spectra obtained from 24 hours samples containing different $\mathrm{C}-\mathrm{N}$ percentage N-CNP sensor platforms along with fluorescence micrograph and (D) Variation in different intracellular components peaks of immobilized HeLa cells based on SERS Raman spectra obtained from 24 hours samples containing different $\mathrm{C}-\mathrm{N}$ percentage N-CNP sensor platforms along fluorescence micrograph with (E) Raman enhancement for DNA peak and protein peak.

Therefore, a significant Raman signal enhancement achieved in presence of N-CNP sensor platforms.

\subsubsection{HeLa cells diagnosis from live SERS Raman spectra}

Relative intensity change of different intracellular components such as protein, DNA and RNA of HeLa cells over the 6-hour interval over the 24 hours incubation period recorded through SERS based Raman signal in comparison to the same intracellular components of fibroblast cells might be the guideline for detection and differentiations of both the cell lines (Figure 6-7). There was an increment of relative intensity of Protein, DNA and RNA peaks of HeLa cells initially from 6-hour to 12 -hour of the incubation period. With the passage of time, the relative intensity showed declining trend for all the intracellular components. However, characteristic opposite behavior was observed for fibroblast cells. The distinctive difference of cell shapes of HeLa and fibroblast cells depicted in fluorescence micrographs (figure 6-7) might shed light on this opposite behavior. Over the passage of time the N-CNP sensor platform might create inhibitive cues for HeLa cells towards growth and proliferate and ended up with rounded structure for Hela. This inhibitive function restricts the regular activities of DNA, RNA and protein of HeLa and resulted in reduced Raman intensity. However, as there were no such activities for fibroblast the growth as well as 
proliferative behavior was positive and resulted in increased relative intensity of the corresponding intracellular components.

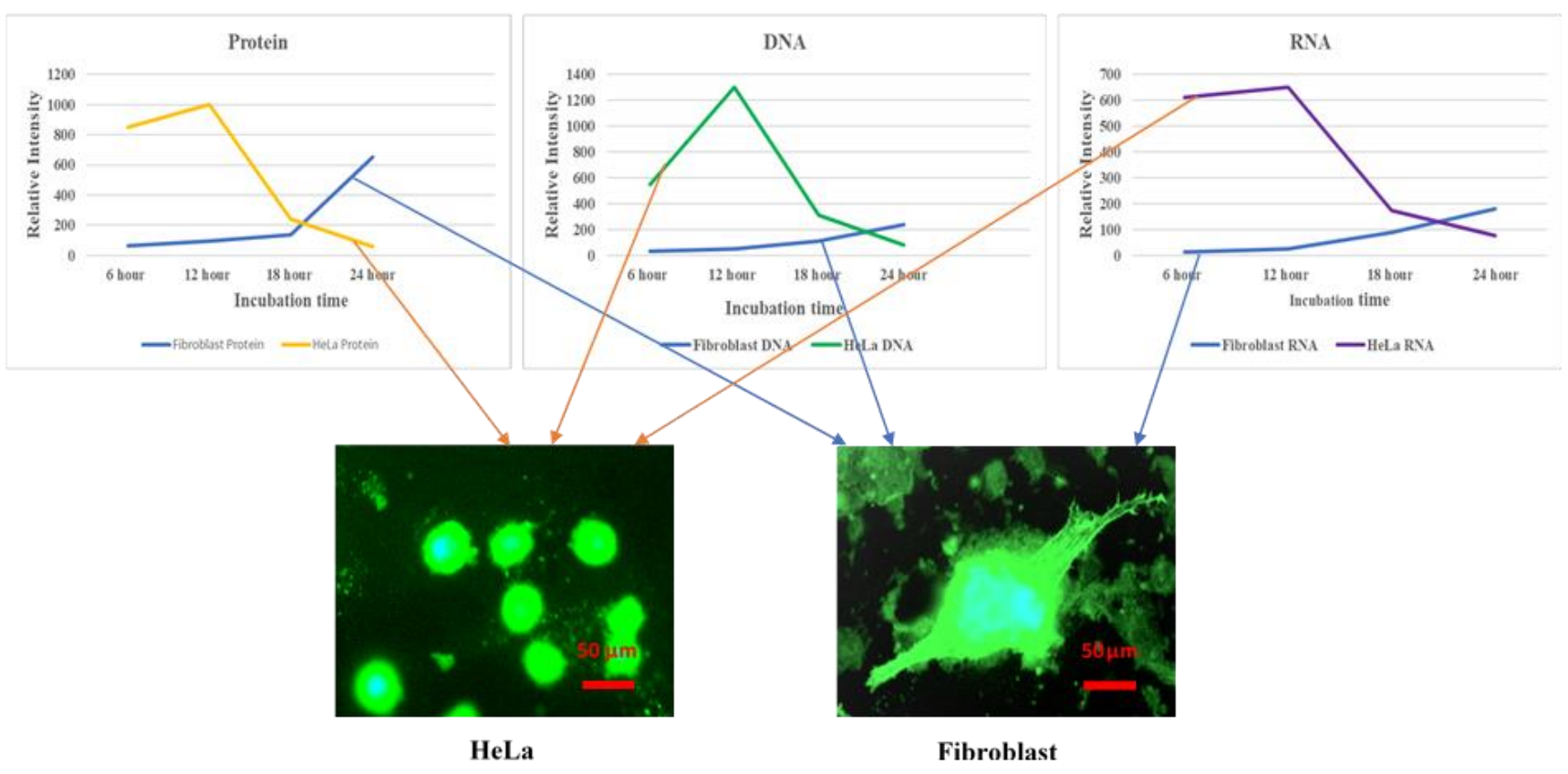

Figure 5-7 Diagnosis of HeLa cells through relative intensity change of protein, DNA and RNA peaks of both HeLa and fibroblast cells from live Raman spectra analysis for 24 hours incubation period with 6 hours intervals.

\subsection{Summary}

SERS based cancer diagnostic approach with Raman spectroscopy supported by carbonaceous nanostructures is a new analytical platform to researchers. However, their individual application as SERS substrates is hindered by their low SERS enhancement and inferior detection sensitivity. This in vitro study exhibited the development of a new nitrogen enriched 3D carbon nanoprobe SERS sensor platform for label free early detection of HeLa cancer cells. The SERS active N-CNP sensor platform showed a great potential for the investigation of intracellular structure components and thereby diagnosis of HeLa cancer cells. This SERS based spectroscopy provided intracellular 
molecular information noninvasively and without any labeling. The N-CNP sensor platform yields reproducible SERS signals with an enhancement factor of $2.10 \times 10^{4}$ with the crystal violet (CV) dye which is in the higher side in comparison to Raman enhancement factors on the surface of monolayer graphene (2 to 17). The created platform showed enhanced microenvironment favorability in terms of both fibroblast and HeLa cell attraction and subsequent adhesion which ultimately reflects its biocompatibility. SERS hot spots were created inside the HeLa and fibroblast cells through internalization of N-CNP unit having physical morphology below ten nanometer NCNP width and thereby achieved SERS based Raman sensing of live cells. The live HeLa and fibroblast cells seeded on N-CNP containing platform was monitored with handheld nano Raman system during the incubation period of 24 hours at an interval of 6 hours. The results depicted peaks related to intracellular biochemical components like proteins, DNA and RNA. The highest Raman signal enhancement achieved for intracellular molecules such as DNA and was almost 6fold in comparison to carbon sensor spectra. Dominance of specific peaks in the Raman spectra profile and the significant relative intensity changes obtained for fibroblast and HeLa for live condition suggested a guideline in detecting and differentiating the HeLa cells in comparison to fibroblast and thereby early diagnosis of Hela cells achieved. The creation of the self-assembled 3D N-CNP sensor platform was done through employment of one step femtosecond laser processing on the non-bio responsive $\mathrm{C}-\mathrm{C}$ bond containing a graphite plate. Both morphology and physicochemical properties of created 3D N-CNP sensor platform varies with the change in ionization energy. N-CNP width shows gradual reduction with the increment of ionization energy. 


\section{Chapter 6}

\section{Summary and Future Research}

\subsection{Summary}

In this dessertation, the formation of a carbon-based nanomaterials in the form of a three dimensional (3D) interwoven nanostructure that exhibits therapeutic and diagnostic application is presented. The unique structure, the physical morphology and physicochemical properties of this carbon nanostrucutre are aided by using the femtosecond pulsed laser ionization with precise control of laser parameter such as laser power, repetation rate, laser pulse width, scanning speed, atmospheric conditions and introduction of $\mathrm{N}_{2}$ gas species to the ion plume. Wide range of novel attributes like bio-active phase, cytophilic-cytotoxic dual functionality, bio-sensitive SERS activeness has been achieved from the synthesized nanocarbons.

Transformation of non-responsive carbon phase of graphite surface to bio-active carbon phase without the post-biological or chemical treatment is presented. Created nanocarbon structures is biocompatible and encourages fibroblast cell adhesion and subsequent vigorous prolefiration. Cell attraction capability irrespective of cell lines along with cell selective citotoxicity lead to high efficiency of cancer therapy and at the same time prolefirative tissue generation for fibroblast indicate the possibility of utilizing the created nanocarbon as scafhold for tissue engineering.

While exploring the diagnostic applications of the nanocarbon materials, the SERS activeness of the interconnected nanocarbon web is identified and hence used as a unique non-plasmonic SERS based bio-sensing platform for in vitro detection and differntiation of HeLa and fibroblast cells. Early diagnosis of cancer is important for saving lives. With a invasion to early stage detection of cancerous HeLa cell in vitro, nitrogen is introduced to the nanocarbon structure. Therefore, the 
nitrogen enriched nanocarbon employs SERS based technique for intracellular sesning. The results reveal the chemical fingurprints of intracellular components both HeLa and fibroblast cells and by which cell differentiation and early diagnosis of HeLa cell has made possible.

The main contribution of this dissertation can be highlighted as follows:

- The development of a new biocompatible 3D carbon nano-network with a bioactive phase that significantly promotes cell adhesion. This cytophilic bio-nanocarbon does not require any subsequent biological/chemical treatments and considerably stimulates cell adhesion and subsequent cell proliferation.

- The carbon nanoribbon network platform having a combination of C-C and C-O bond architecture has dual functionality i.e. cytophilic to fibroblasts but cytotoxic to HeLa cells. The said platform acts as a favorable attractor and a proliferation-promoting substrate for both fibroblasts and HeLa cells. With the passage of time it induces cell-specific anticancer cytotoxicity on HeLa cells and lead to cell death (apoptosis) but no harm on mammalian fibroblast cells and develops tissue like structures.

- SERS active 3D interconnected nanocarbon web is introduced as a unique non-plasmonic SERS based bio-sensing platform for in-vitro detection and differentiation of HeLa and fibroblast cells. The sub-10 nanometer structure of the said nanocarbon has selfinternalization capacity which results in live cell Raman sensing. Individual detection of HeLa and fibroblast cells from chemical fingerprints of intracellular components like DNA/RNA, protein and lipids of both cell lines have been revealed through Raman spectroscopic peak differences. Additionally, there is a guideline for identification of each cell health from spectral responses. 
- Finally, nitrogen enrichment to the 3D interconnected nanocarbon structure introduce exceptional attributes to nitrogen enriched 3D carbon nanoprobe platform and demonstrate a label free non-plasmonic SERS based unique technique of early detection of HeLa cancer cells by intracellular sensing. This platform demonstrates favorable microenvironment for cell adhesion and SERS hot spots is created inside the HeLa and fibroblast cells through internalization of nitrogen enriched 3D carbon nanoprobe unit and thereby achieves SERS based live cells Raman sensing. The revealed fingerprints of the intracellular components (protein, DNA, RNA etc.) of HeLa and fibroblasts cells guide early detection of HeLa cells and thereby cancer diagnosis.

\subsection{Future Research}

The created carbonaceous nanostructures exhibited cytophilic-cytotoxic dual functionality and SERS enhancement. The versatility of these nanocarbon can be envisioned with a different cancer cell lines such as breast cancer cells. Nitrogen enriched nanocarbon platform has been explored as SERS based cancer detection probe. Some preliminary results suggest that the said nanostructure might be acted as "anti-cancer" nano-therapeutic agents. The scopes of the SERS based Raman spectroscopic sensing method may broden in the detection of bacterial infection, specifically E. Coli Detection. 


\section{Appendices}

\section{Appendix 1}

\section{Raman Enhancement Factor calculation:}

The Raman enhancement factor $(\mathrm{EF})$ for the interconnected nanocarbon web is calculated experimentally using the below mentioned equation which is in accordance with most accepted literature ${ }^{1-3}$.

$\mathrm{EF}=\frac{I_{\text {nanostructure }} / N_{\text {surface }}}{I_{\text {substrate }} / N_{\text {bulk }}}$

I $_{\text {nanostructure }}$ represent the Raman SERS intensity on the interconnected nanocarbon web, I substrate represent the Raman intensity on graphite substrate. $\mathrm{N}_{\text {bulk }}$ represent the number of molecules under the confocal volume and $\mathrm{N}_{\text {surface }}$ represents the number of molecules responsible for he SERS response. A $10 \mu$ l volume of both dyes is added to the interconnected nanocarbon web and graphite substrate for assessing SERS response. $\mathrm{I}_{\text {nanostructure }}$ and $\mathrm{I}_{\text {substrate }}$ are measured at $\mathrm{D}$ band and $\mathrm{G}$ band as both the dyes molecule showed strong vibrational mode at D band and $\mathrm{G}$ band and is used for EF calculations. $\mathrm{N}_{\text {bulk }}$ is calculated using the following equation

$N_{\text {bulk }}=\pi r^{2} h c N_{A}$

Here, $\mathrm{N}_{\mathrm{A}}$ is Avogadro's constant, $\mathrm{r}$ represents the Raman laser spot radius $(1.5 \mu \mathrm{m}), \mathrm{h}$ is the half of the depth of field (DOF) of a 50X magnified laser used in this study $(2 \mu \mathrm{m})$, and $\mathrm{c}$ is the dye concentration $\left(1 \times 10^{-3} \mathrm{M}\right)$. To calculate $\mathrm{N}_{\text {surface }}$ the following equation is used

$N_{\text {surface }}=S A_{\text {eff }}\left(C_{\text {ads }} N_{A} \frac{1000 L}{1 m^{3}}\right)^{2 / 3}$

Where, $\mathrm{SA}_{\text {eff }}$ is the effective surface area of nanocarbon structure within the Raman laser interaction volume, $\mathrm{C}_{\mathrm{ads}}$ is the surface adsorption of the dye molecule and $\mathrm{N}_{\mathrm{A}}$ is Avogadro's constant. Calculation of both surface area and dye absorption on nanocarbon structure are required 
to calculate $\mathrm{N}_{\text {surface. }}$ Based on the previous works of Maznichenko et al. ${ }^{4}$ and Powell J.A. et al. ${ }^{5}$ some conservative estimates for nanonetwork were made in calculating $\mathrm{N}_{\text {surface }}$. The nanocarbon network density is assumed to be identical to bulk graphite $\rho=1.82 \mathrm{~g} / \mathrm{m}^{3}$ (manufacture's data sheet Graphtec LLC) and BET surface area as $32.4 \mathrm{~m}^{2} / \mathrm{g}$ from literature ${ }^{6}$. The SERS experiments were performed instantly after application of both the dyes on interconnected nanocarbon web and on graphite substrate. There is a requirement of identifying the number of dye molecules adsorb onto the surface of nanonetwork to estimate the number of molecules that contribute to Raman enhancement from the nanocarbon structure. A similarity relationship is established as it is not possible to weigh cubic micrometer volumes. It is assumed that only the molecules present within the interaction volume of the Raman laser contribute to Raman enhancement for both $\mathrm{N}_{\text {bulk }}$ and $\mathrm{N}_{\text {surface }}$ calculation.

\section{Reference}

(1) Martinez-Garcia, M. M.; Cardoso-Avila, P. E.; Pichardo-Molina, J. L. Concave Gold Nanocubes on Al-6063 Alloy as a Simple and Efficient SERS Substrate. Colloids Surf. Physicochem. Eng. Asp. 2016, 493, 66-73.

(2) Yan, Z.; Du, W.; Tu, L.; Gu, P.; Huang, Z.; Zhan, P.; Liu, F.; Wang, Z. A Facile HighPerformance SERS Substrate Based on Broadband near-Perfect Optical Absorption. J. Raman Spectrosc. 2015, 46 (9), 795-801.

(3) Le Ru, E. C.; Blackie, E.; Meyer, M.; Etchegoin, P. G. Surface Enhanced Raman Scattering Enhancement Factors: A Comprehensive Study. J. Phys. Chem. C 2007, 111 (37), 1379413803.

(4) Maznichenko, D.; Venkatakrishnan, K.; Tan, B. Stimulating Multiple SERS Mechanisms by a Nanofibrous Three-Dimensional Network Structure of Titanium Dioxide (TiO2). J. Phys. Chem. C 2013, 117 (1), 578-583.

(5) Powell, J. A.; Venkatakrishnan, K.; Tan, B. Programmable SERS Active Substrates for Chemical and Biosensing Applications Using Amorphous/crystalline Hybrid Silicon Nanomaterial. Sci. Rep. 2016, 6, 19663. 
(6) He, Z.; Liu, L.; Gao, C.; Zhou, Z.; Liang, X.; Lei, Y.; He, Z.; Liu, S. Carbon Nanofibers Grown on the Surface of Graphite Felt by Chemical Vapour Deposition for Vanadium Redox Flow Batteries. RSC Adv. 2013, 3 (43), 19774-19777. 


\section{Appendix 2}

a.

Nanocarbon uptake for medium C-O concentration interconnected nanocarbon

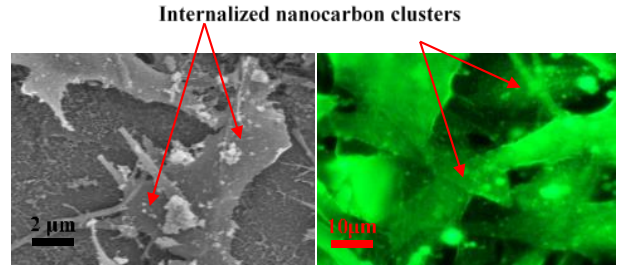

Fibroblast

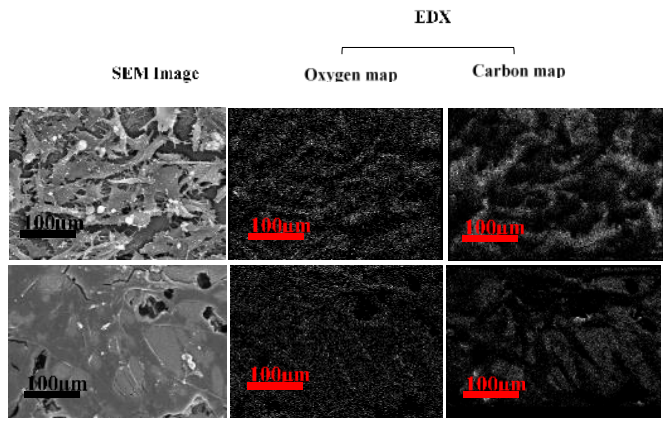

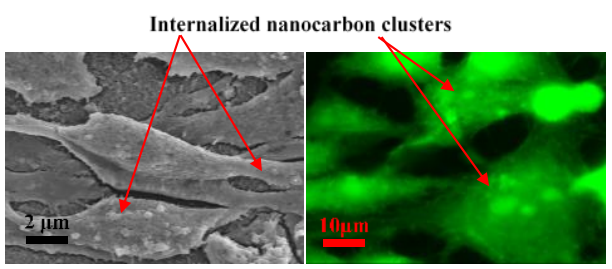

Hel aa

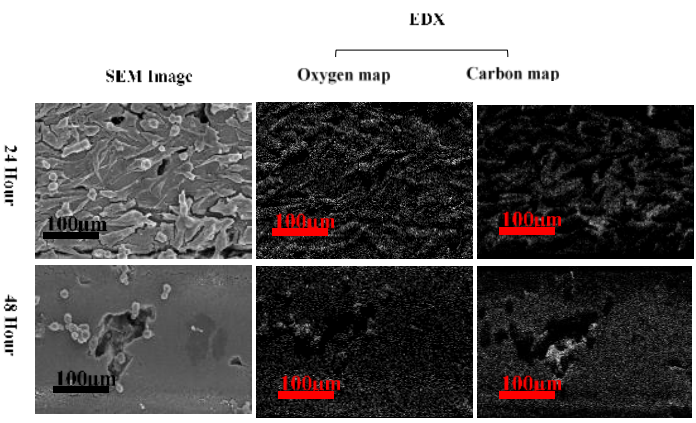

b.

Nanocarbon uptake for Low C-O concentration interconnected nanocarbon web

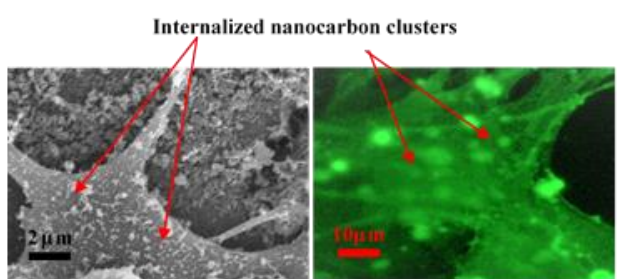

Fibroblast

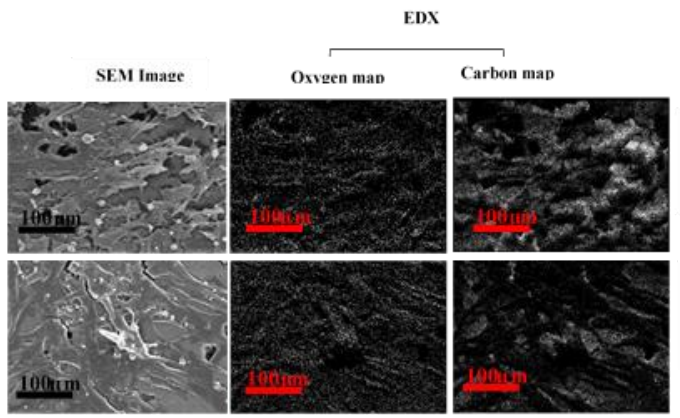

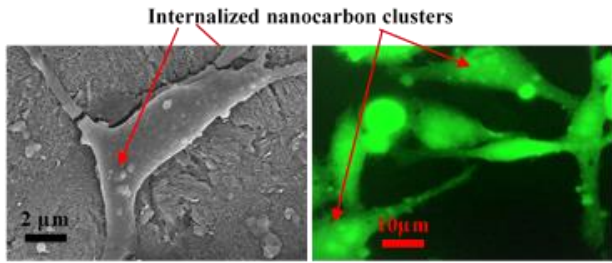

HeLa

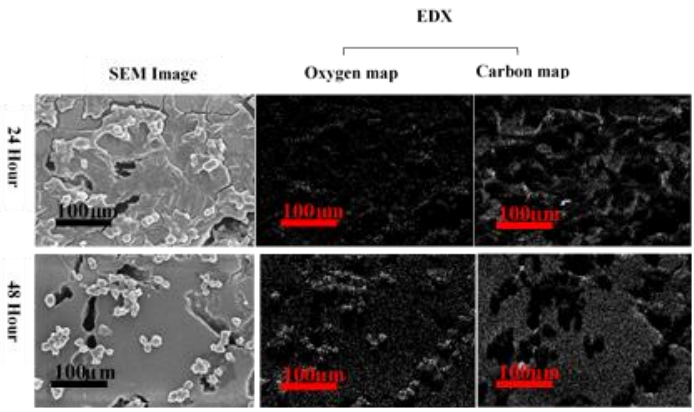




\section{Appendix 3}

a. Table S1: Peak assignments for Raman spectra data recorded for fibroblast cells

\begin{tabular}{|c|c|c|c|}
\hline $\begin{array}{l}\text { Wave number } \\
\qquad\left(\mathrm{cm}^{-1}\right)\end{array}$ & Raman Peak assignment & $\begin{array}{l}\text { Wave number }\left(\mathrm{cm}^{-1}\right) \\
\text { (for live cell on high } \mathrm{C}-\mathrm{O} \\
\text { concentration } \\
\text { nanocarbon platform } \\
\text { after } 24 \text { hours) }\end{array}$ & $\begin{array}{l}\text { Wave number }\left(\mathrm{cm}^{-1}\right) \\
\text { (for cells fixed on high } \\
\text { C-O concentration } \\
\text { nanocarbon platform } \\
\text { after } 24 \text { hours) }\end{array}$ \\
\hline 415 & Phosphatidylinositol (lipid) & - & 400 \\
\hline 477 & Polysaccharides, amylose & - & 472 \\
\hline 662 & $\begin{array}{l}\text { C-S stretching mode of } \\
\text { cystine (collagen type I) }\end{array}$ & - & 660 \\
\hline 678 & $\begin{array}{l}\text { Ring breathing modes in the } \\
\text { DNA bases }\end{array}$ & 680 & \\
\hline 788 & DNA & 792 & \\
\hline 802 & $\begin{array}{l}\text { Uracil-based ring breathing } \\
\text { mode }\end{array}$ & - & 804 \\
\hline 974 & RNA & - & 976 \\
\hline 1023 & Glycogen & - & 1024 \\
\hline 1235 & Amide III (Proteins) & 1232 & - \\
\hline 1291 & Cytosine (DNA) & 1292 & - \\
\hline 1465 & Lipids & 1464 & - \\
\hline 1480 & Amide II (Proteins) & - & 1480 \\
\hline 1600 & Amide I (Proteins) & 1600 & - \\
\hline 1697 & Amide I (Proteins) & 1696 & 1692 \\
\hline 1756 & Lipids & - & 1768 \\
\hline 2095 & $\mathrm{C}-\mathrm{N}$ stretch & 1864 & - \\
\hline \multirow[t]{9}{*}{$2300-3800$} & $\begin{array}{l}\text { Region of the } \mathrm{OH}-\mathrm{NH}-\mathrm{CH} \\
\text { stretching vibrations (Lipids) }\end{array}$ & 2328 & - \\
\hline & & 2384 & \\
\hline & & & 2416 \\
\hline & & 2440 & \\
\hline & & & 2500 \\
\hline & & 2548 & \\
\hline & & & 2620 \\
\hline & & 2752 & 2752 \\
\hline & & & 2828 \\
\hline 2876 & $\begin{array}{l}\mathrm{CH} 2 \text { asymmetric stretch of } \\
\text { lipids and proteins }\end{array}$ & 2876 & 2880 \\
\hline
\end{tabular}


b. Table S2: Peak assignments for Raman spectra data recorded for HeLa cells

\begin{tabular}{|c|c|c|c|}
\hline $\begin{array}{l}\text { Wave number } \\
\qquad\left(\mathrm{cm}^{-1}\right)\end{array}$ & Raman Peak assignment & $\begin{array}{l}\text { Wave number }\left(\mathrm{cm}^{-1}\right) \\
\text { (for live cell on high C-O } \\
\text { concentration } \\
\text { nanocarbon platform } \\
\text { after } 24 \text { hours) }\end{array}$ & $\begin{array}{l}\text { Wave number }\left(\mathrm{cm}^{-1}\right) \\
\text { (for cells fixed on high } \\
\text { C-O concentration } \\
\text { nanocarbon platform } \\
\text { after } 24 \text { hours) }\end{array}$ \\
\hline 415 & Phosphatidylinositol (lipid) & 408 & 404 \\
\hline $447 / 54$ & Ring torsion of phenyl (2) & 456 & - \\
\hline 477 & Polysaccharides, amylose & - & 488 \\
\hline 573 & $\begin{array}{l}\text { Tryptophan/cytosine, } \\
\text { guanine (DNA/RNA) }\end{array}$ & 552 & 560 \\
\hline 678 & C, T, G (DNA/RNA) & 604 & 680 \\
\hline $\begin{array}{l}811 \\
827\end{array}$ & $\begin{array}{l}\text { O-P-O stretching RNA } \\
\text { DNA }\end{array}$ & 828 & 818 \\
\hline 974 & $\begin{array}{l}\text { Ribose vibration, one of the } \\
\text { distinct RNA modes }\end{array}$ & - & 976 \\
\hline 1022 & Glycogen & - & 1020 \\
\hline 1104 & Phenylalanine (proteins) & - & 1104 \\
\hline 1155 & $\begin{array}{l}\mathrm{C}-\mathrm{C} \text { and } \mathrm{C}-\mathrm{N} \text { stretching of } \\
\text { proteins }\end{array}$ & - & 1160 \\
\hline $1247 / 8$ & $\begin{array}{l}\text { Guanine, cytosine }\left(\mathrm{NH}_{2}\right) \\
\text { (DNA) }\end{array}$ & - & 1248 \\
\hline 1313 & $\begin{array}{l}\mathrm{CH}_{3} \mathrm{CH}_{2} \text { twisting mode of } \\
\text { collagen/lipid }\end{array}$ & 1312 & \\
\hline 1453 & Proteins & 1452 & - \\
\hline 1593 & $\begin{array}{l}\mathrm{C}=\mathrm{N} \text { and } \mathrm{C}=\mathrm{C} \text { stretching in } \\
\text { quinoid ring }\end{array}$ & 1596 & \\
\hline 1667 & Proteins & - & 1668 \\
\hline $1716-41$ & $\mathrm{C}=\mathrm{O}$ & - & 1720 \\
\hline 1756 & Lipids & - & 1776 \\
\hline 2095 & $\mathrm{C}-\mathrm{N}$ stretch & 2096 & 2016 \\
\hline 2225 & $\mathrm{C}=\mathrm{N}$ & 2216 & 2216 \\
\hline \multirow[t]{9}{*}{$2300-3800$} & $\begin{array}{l}\text { Region of the OH-NH-CH } \\
\text { stretching vibrations (Lipids) }\end{array}$ & - & 2376 \\
\hline & & - & 2416 \\
\hline & & 2480 & - \\
\hline & & - & 2508 \\
\hline & & 2548 & 2552 \\
\hline & & 2652 & 2624 \\
\hline & & - & 2720 \\
\hline & & - & 2752 \\
\hline & & 2824 & - \\
\hline 2876 & $\begin{array}{l}\mathrm{CH}_{2} \text { asymmetric stretch of } \\
\text { lipids and proteins }\end{array}$ & 2872 & 2876 \\
\hline
\end{tabular}


c Table S3: Raman signal enhancement for DNA, protein and lipid peaks for live Fibroblast cells

\begin{tabular}{|c|r|r|r|}
\hline Incubation time & \multicolumn{3}{|c|}{ Raman signal enhancement } \\
\hline 6 Hour & DNA & Protein & Lipid \\
\hline 12 Hour & 2 & 1 & 2 \\
\hline 18 Hour & 1 & 1 & 2 \\
\hline 24 Hour & 3 & 4 & 4 \\
\hline
\end{tabular}

d. Table S4: Raman signal enhancement for DNA, protein and lipid peaks for live HeLa cells

\begin{tabular}{|c|c|c|c|}
\hline Incubation time & \multicolumn{3}{|c|}{ Raman signal enhancement } \\
\hline & DNA & Protein & Lipid \\
\hline 6 Hour & 3.10 & 3.17 & 3.49 \\
\hline 12 Hour & 3.68 & 4.03 & 4.09 \\
\hline 18 Hour & 4.21 & 4.71 & 4.83 \\
\hline 24 Hour & 6.12 & 5.66 & 5.21 \\
\hline
\end{tabular}




\section{Appendix 4}

a. Table S3: Peak assignments for Raman spectra data recorded for fibroblast cells

\begin{tabular}{|c|c|c|c|}
\hline $\begin{array}{l}\text { Wave number } \\
\qquad\left(\mathrm{cm}^{-1}\right)\end{array}$ & Raman Peak assignment & $\begin{array}{l}\text { Wave number }\left(\mathrm{cm}^{-1}\right) \\
\text { (for live fibroblast cell on } \\
\text { N-CNP sensor platform) }\end{array}$ & $\begin{array}{l}\text { Wave number }\left(\mathrm{cm}^{-1}\right) \\
\text { (for fibroblast cells } \\
\text { fixed on } \mathrm{N}-\mathrm{CNP} \\
\text { sensor platform) }\end{array}$ \\
\hline $667 / 9$ & T, G (DNA/RNA) & 656 & - \\
\hline 776 & Phosphatidylinositol (RNA) & 776 & \\
\hline 752 & DNA & - & 756 \\
\hline 780 & $\begin{array}{l}\text { Uracil based ring breathing } \\
\text { mode (RNA) }\end{array}$ & 780 & - \\
\hline 800 & DNA & 800 & - \\
\hline 810 & $\begin{array}{l}\text { Phosphodiester (Z-marker) } \\
\text { (DNA) }\end{array}$ & 808 & \\
\hline 812 & $\begin{array}{l}\text { Phosphodiester (Z-marker) } \\
\text { (DNA) }\end{array}$ & - & 812 \\
\hline 815 & $\begin{array}{l}\text { Proline, hydroxyproline, } \\
\text { tyrosine (DNA) }\end{array}$ & 816 & - \\
\hline 1208 & $\begin{array}{l}\mathrm{n}(\mathrm{C}-\mathrm{C} 6 \mathrm{H} 5), \text { tryptophan, } \\
\text { phenylalanine (protein } \\
\text { assignment) }\end{array}$ & 1212 & - \\
\hline 1230 & Amide III (proteins) & 1232 & - \\
\hline 1296 & CH2 deformation (DNA) & 1296 & - \\
\hline 1470 & C-N stretching (Protein) & 1472 & \\
\hline 1600 & $\begin{array}{l}\text { Amide I band of proteins-Due } \\
\text { to } \mathrm{C}-\mathrm{O} \text { stretching }\end{array}$ & 1596 & 1576 \\
\hline 1640 & Amide I (proteins) & 1640 & - \\
\hline
\end{tabular}


b. Table S4: Peak assignments for Raman spectra data recorded for HeLa cells

\begin{tabular}{|c|c|c|c|}
\hline $\begin{array}{l}\text { Wave number } \\
\qquad\left(\mathrm{cm}^{-1}\right)\end{array}$ & Raman Peak assignment & $\begin{array}{l}\text { Wave number }\left(\mathrm{cm}^{-1}\right) \\
\text { (for live HeLa cell on } \mathrm{N} \text { - } \\
\text { CNP sensor platform) }\end{array}$ & $\begin{array}{l}\text { Wave number }\left(\mathrm{cm}^{-1}\right) \\
\text { (for cells fixed on } \mathrm{N} \text { - } \\
\text { CNP sensor platform) }\end{array}$ \\
\hline $667 / 9$ & T, G (DNA/RNA) & 668 & - \\
\hline 752 & DNA & 752 & 748 \\
\hline 766 & $\begin{array}{l}\text { Pyrimidine ring breathing } \\
\text { mode (RNA }\end{array}$ & 764 & - \\
\hline 785 & $\begin{array}{l}\mathrm{U}, \mathrm{T}, \mathrm{C} \text { (ring breathing modes } \\
\text { in the DNA/RNA bases) }\end{array}$ & 784 & 760 \\
\hline 800 & DNA & 800 & - \\
\hline 802 & $\begin{array}{l}\text { Uracil-based ring breathing } \\
\text { mode (DNA) }\end{array}$ & 804 & \\
\hline 812 & $\begin{array}{l}\text { Phosphodiester } \quad \text { (Z-marker) } \\
\text { (DNA) }\end{array}$ & 812 & 812 \\
\hline 826 & O-P-O stretching DNA/RNA & 824 & - \\
\hline 1200 & Amide III (proteins) & 1200 & - \\
\hline 1280 & Amide III (proteins) & 1280 & - \\
\hline 1288 & $\begin{array}{l}\text { Phosphodiester groups in } \\
\text { nucleic acids }\end{array}$ & 1288 & - \\
\hline 1600 & $\begin{array}{l}\text { Amide I band of proteins-Due } \\
\text { to } \mathrm{C}-\mathrm{O} \text { stretching }\end{array}$ & 1596 & 1576 \\
\hline 1656 & Amide I (proteins) & 1656 & - \\
\hline
\end{tabular}




\section{References}

1-1. Li, R.; Wu, R.; Zhao, L.; Hu, Z.; Guo, S.; Pan, X.; Zou, H. Folate and Iron Difunctionalized Multiwall Carbon Nanotubes as Dual-Targeted Drug Nanocarrier to Cancer Cells. Carbon 2011, $49(5), 1797-1805$.

1-2. Stancu, E. C.; Stanciuc, A.-M.; Vizireanu, S.; Luculescu, C.; Moldovan, L.; Amine Achour;

Dinescu, G. Plasma Functionalization of Carbon Nanowalls and Its Effect on Attachment of Fibroblast-like Cells. J. Phys. Appl. Phys. 2014, 47 (26), 265203.

1-3. Lee, W. C.; Lim, C. H. Y. X.; Shi, H.; Tang, L. A. L.; Wang, Y.; Lim, C. T.; Loh, K. P. Origin of Enhanced Stem Cell Growth and Differentiation on Graphene and Graphene Oxide. ACS Nano 2011, 5 (9), 7334-7341.

1-4. Talukdar, Y.; Rashkow, J. T.; Lalwani, G.; Kanakia, S.; Sitharaman, B. The Effects of Graphene Nanostructures on Mesenchymal Stem Cells. Biomaterials 2014, 35 (18), 4863-4877.

1-5. Harrison, B. S.; Atala, A. Carbon Nanotube Applications for Tissue Engineering. Biomaterials 2007, 28 (2), 344-353.

1-6. Kaya, C.; Singh, I.; Boccaccini, A. R. Multi-Walled Carbon Nanotube-Reinforced Hydroxyapatite Layers on Ti6Al4V Medical Implants by Electrophoretic Deposition (EPD). Adv. Eng. Mater. 2008, 10 (1-2), 131-138.

1-7. Jang, H.; Kim, Y.-K.; Kwon, H.-M.; Yeo, W.-S.; Kim, D.-E.; Min, D.-H. A Graphene-Based Platform for the Assay of Duplex-DNA Unwinding by Helicase. Angew. Chem. 2010, 122 (33), $5839-5843$.

1-8. De La Zerda, A.; Zavaleta, C.; Keren, S.; Vaithilingam, S.; Bodapati, S.; Liu, Z.; Levi, J.;

Smith, B. R.; Ma, T.-J.; Oralkan, O.; et al. Carbon Nanotubes as Photoacoustic Molecular Imaging Agents in Living Mice. Nat. Nanotechnol. 2008, 3 (9), 557-562. 
1-9. Liu, Z.; Robinson, J. T.; Sun, X.; Dai, H. PEGylated Nanographene Oxide for Delivery of Water-Insoluble Cancer Drugs. J. Am. Chem. Soc. 2008, 130 (33), 10876-10877.

1-10. Hu, W.; Peng, C.; Luo, W.; Lv, M.; Li, X.; Li, D.; Huang, Q.; Fan, C. Graphene-Based Antibacterial Paper. ACS Nano 2010, 4 (7), 4317-4323.

1-11. Baughman, R. H.; Zakhidov, A. A.; Heer, W. A. de. Carbon Nanotubes--the Route Toward Applications. Science 2002, 297 (5582), 787-792.

1-12. Allen, M. J.; Tung, V. C.; Kaner, R. B. Honeycomb Carbon: A Review of Graphene. Chem. Rev. 2010, $110(1), 132-145$.

1-13. Namgung, S.; Kim, T.; Baik, K. Y.; Lee, M.; Nam, J.-M.; Hong, S. Fibronectin-CarbonNanotube Hybrid Nanostructures for Controlled Cell Growth. Small 2011, 7 (1), 56-61.

1-14. Ryoo, S.-R.; Kim, Y.-K.; Kim, M.-H.; Min, D.-H. Behaviors of NIH-3T3 Fibroblasts on Graphene/Carbon Nanotubes: Proliferation, Focal Adhesion, and Gene Transfection Studies. ACS Nano 2010, 4 (11), 6587-6598.

1-15. Aryaei, A.; Jayatissa, A. H.; Jayasuriya, A. C. The Effect of Graphene Substrate on Osteoblast Cell Adhesion and Proliferation. J. Biomed. Mater. Res. A 2014, 102 (9), 3282-3290.

1-16. Prato, M.; Kostarelos, K.; Bianco, A. Functionalized Carbon Nanotubes in Drug Design and Discovery. Acc. Chem. Res. 2007, 41 (1), 60-68.

1-17. Mendes, R. G.; Bachmatiuk, A.; Büchner, B.; Cuniberti, G.; Rümmeli, M. H. Carbon Nanostructures as Multi-Functional Drug Delivery Platforms. J Mater Chem B 2013, 1 (4), 401428.

1-18. Jesion, I.; Skibniewski, M.; Skibniewska, E.; Strupiński, W.; Szulc-Dąbrowska, L.; Krajewska, A.; Pasternak, I.; Kowalczyk, P.; Pińkowski, R. Graphene and Carbon 
Nanocompounds: Biofunctionalization and Applications in Tissue Engineering. Biotechnol. Biotechnol. Equip. 2015, 29 (3), 415-422.

1-19. Xu, Y.; Mahmood, M.; Li, Z.; Dervishi, E.; Trigwell, S.; Zharov, V. P.; Ali, N.; Viney Saini; Biris, A. R.; Lupu, D.; et al. Cobalt Nanoparticles Coated with Graphitic Shells as Localized Radio Frequency Absorbers for Cancer Therapy. Nanotechnology 2008, 19 (43), 435102.

1-20. Batra, B.; Lata, S.; Pundir, C. Construction of an Improved Amperometric Acrylamide

Biosensor Based on Hemoglobin Immobilized onto Carboxylated Multi-Walled Carbon Nanotubes/iron Oxide Nanoparticles/chitosan Composite Film. Bioprocess Biosyst. Eng. 2013, 36 (11), 1591-1599.

1-21. Shan, C.; Yang, H.; Han, D.; Zhang, Q.; Ivaska, A.; Niu, L. Graphene/AuNPs/chitosan Nanocomposites Film for Glucose Biosensing. Biosens. Bioelectron. 2010, 25 (5), 1070-1074.

1-22. Kailashiya, J.; Singh, N.; Singh, S. K.; Agrawal, V.; Dash, D. Graphene Oxide-Based Biosensor for Detection of Platelet-Derived Microparticles: A Potential Tool for Thrombus Risk Identification. Biosens. Bioelectron. 2015, 65, 274-280.

1-23. Basu, P. K.; Indukuri, D.; Keshavan, S.; Navratna, V.; Vanjari, S. R. K.; Raghavan, S.;

Bhat, N. Graphene Based E. Coli Sensor on Flexible Acetate Sheet. Sens. Actuators B Chem. 2014, $190,342-347$.

1-24. Tiwari, I.; Singh, M.; Pandey, C. M.; Sumana, G. Electrochemical Genosensor Based on Graphene Oxide Modified Iron Oxide-chitosan Hybrid Nanocomposite for Pathogen Detection. Sens. Actuators B Chem. 2015, 206, 276-283.

1-25. Huang, H.; Bai, W.; Dong, C.; Guo, R.; Liu, Z. An Ultrasensitive Electrochemical DNA Biosensor Based on graphene/Au Nanorod/polythionine for Human Papillomavirus DNA Detection. Biosens. Bioelectron. 2015, 68, 442-446. 
1-26. Bhirde, A. A.; Liu, G.; Jin, A.; Iglesias-Bartolome, R.; Sousa, A. A.; Leapman, R. D.; Gutkind, J. S.; Lee, S.; Chen, X. Combining Portable Raman Probes with Nanotubes for Theranostic Applications. Theranostics 2011, 1, 310.

1-27. Manikandan, M.; Abdelhamid, H. N.; Talib, A.; Wu, H.-F. Facile Synthesis of Gold Nanohexagons on Graphene Templates in Raman Spectroscopy for Biosensing Cancer and Cancer Stem Cells. Biosens. Bioelectron. 2014, 55, 180-186.

1-28. Sengupta, R.; Bhattacharya, M.; Bandyopadhyay, S.; Bhowmick, A. K. A Review on the Mechanical and Electrical Properties of Graphite and Modified Graphite Reinforced Polymer Composites. Prog. Polym. Sci. 2011, 36 (5), 638-670.

1-29. Szabó, A.; Perri, C.; Csató, A.; Giordano, G.; Vuono, D.; Nagy, J. B. Synthesis Methods of Carbon Nanotubes and Related Materials. Materials 2010, 3 (5), 3092-3140.

1-30. Al-Hamaoy, A.; Chikarakara, E.; Jawad, H.; Gupta, K.; Kumar, D.; Rao, M. R.; Krishnamurthy, S.; Morshed, M.; Fox, E.; Brougham, D. Liquid Phase-Pulsed Laser Ablation: A Route to Fabricate Different Carbon Nanostructures. Appl. Surf. Sci. 2014, 302, 141-144.

1-31. Radhakrishnan, G.; Adams, P. M.; Bernstein, L. S. Room-Temperature Deposition of Carbon Nanomaterials by Excimer Laser Ablation. Thin Solid Films 2006, 515 (3), 1142-1146.

1-32. Azami, T.; Kasuya, D.; Yoshitake, T.; Kubo, Y.; Yudasaka, M.; Ichihashi, T.; Iijima, S. Production of Small Single-Wall Carbon Nanohorns by CO2 Laser Ablation of Graphite in NeGas Atmosphere. Carbon 2007, 45 (6), 1364-1367.

2-1. Harrison, B. S. \& Atala, A. Carbon nanotube applications for tissue engineering. Biomaterials 28, 344-353 (2007).

2-2. Talukdar, Y., Rashkow, J. T., Lalwani, G., Kanakia, S. \& Sitharaman, B. The effects of graphene nanostructures on mesenchymal stem cells. Biomaterials 35, 4863-4877 (2014). 
2-3. Kaya, C., Singh, I. \& Boccaccini, A. R. Multi-walled Carbon Nanotube-Reinforced Hydroxyapatite Layers on Ti6Al4V Medical Implants by Electrophoretic Deposition (EPD). Adv. Eng. Mater. 10, 131-138 (2008).

2-4. Jang, H. et al. A Graphene-Based Platform for the Assay of Duplex-DNA Unwinding by Helicase. Angew. Chem. 122, 5839-5843 (2010).

2-5. De La Zerda, A. et al. Carbon nanotubes as photoacoustic molecular imaging agents in living mice. Nat. Nanotechnol. 3, 557-562 (2008).

2-6. Liu, Z., Robinson, J. T., Sun, X. \& Dai, H. PEGylated Nanographene Oxide for Delivery of Water-Insoluble Cancer Drugs. J. Am.Chem. Soc. 130, 10876-10877 (2008).

2-7. Hu, W. et al. Graphene-Based Antibacterial Paper. ACS Nano 4, 4317-4323 (2010).

2-8. Cai, N. et al. Modulating cell adhesion dynamics on carbon nanotube monolayer engineered with extracellular matrix proteins. ACS Appl. Mater. Interfaces 2, 1038-1047 (2010).

2-9. Luo, N., Ni, D., Yue, H., Wei, W. \& Ma, G. Surface-engineered graphene navigate divergent biological outcomes toward macrophages. ACS Appl. Mater. Interfaces 7, 5239-5247 (2015).

2-10. Smart, S. K., Cassady, A. I., Lu, G. Q. \& Martin, D. J. The biocompatibility of carbon nanotubes. Carbon 44, 1034-1047 (2006).

2-11. Kalbacova, M., Broz, A., Kong, J. \& Kalbac, M. Graphene substrates promote adherence of human osteoblasts and mesenchymal stromal cells. Carbon 48, 4323-4329 (2010).

2-12. Jesion, I. et al. Graphene and carbon nanocompounds: biofunctionalization and applications in tissue engineering. Biotechnol. Biotechnol. Equip. 29, 415-422 (2015).

2-13. A. F. ISMAIL, P. S. G. A review of purification techniques for carbon nanotubes. Nano 3, (2008). 
2-14. Liu, Y., Zhao, Y., Sun, B. \& Chen, C. Understanding the toxicity of carbon nanotubes. Acc. Chem. Res. 46, 702-713 (2012).

2-15. Mottaghitalab, F. et al. The effect of fibronectin on structural and biological properties of single walled carbon nanotube. Appl. Surf. Sci. 339, 85-93 (2015).

2-16. Correa-Duarte, M. A. et al. Fabrication and Biocompatibility of Carbon Nanotube-Based 3D Networks as Scaffolds for Cell Seeding and Growth. Nano Lett. 4, 2233-2236 (2004).

2-17. Ryoo, S.-R., Kim, Y.-K., Kim, M.-H. \& Min, D.-H. Behaviors of NIH-3T3 Fibroblasts on Graphene/Carbon Nanotubes: Proliferation, Focal Adhesion, and Gene Transfection Studies. ACS Nano 4, 6587-6598 (2010).

2-18. Aryaei, A., Jayatissa, A. H. \& Jayasuriya, A. C. The effect of graphene substrate on osteoblast cell adhesion and proliferation. J. Biomed. Mater. Res. A 102, 3282-3290 (2014).

2-19. Depan, D., Girase, B., Shah, J. S. \& Misra, R. D. K. Structure-process-property relationship of the polar graphene oxide-mediated cellular response and stimulated growth of osteoblasts on hybrid chitosan network structure nanocomposite scaffolds. Acta Biomater. 7, 3432-3445 (2011). 2-20. Girase, B., Shah, J. S. \& Misra, R. D. K. Cellular mechanics of modulated osteoblasts functions in graphene oxide reinforced elastomers. Adv. Eng. Mater. 14, B101-B111 (2012).

2-21. Shan, C. et al. Water-soluble graphene covalently functionalized by biocompatible poly-Llysine. Langmuir 25, 12030-12033 (2009).

2-22. Zhang, Y. et al. Cytotoxicity Effects of Graphene and Single-Wall Carbon Nanotubes in Neural Phaeochromocytoma-Derived PC12 Cells. ACS Nano 4, 3181-3186 (2010).

2-23. Goenka, S., Sant, V. \& Sant, S. Graphene-based nanomaterials for drug delivery and tissue engineering. J. Controlled Release 173, 75-88 (2014). 
2-24. Kumar, S. et al. Chemical functionalization of graphene to augment stem cell osteogenesis and inhibit biofilm formation on polymer composites for orthopedic applications. ACS Appl. Mater. Interfaces 7, 3237-3252 (2015).

2-25. Tavangar, A., Tan, B. \& Venkatakrishnan, K. Study of the formation of 3-D titania nanofibrous structure by MHz femtosecond laser in ambient air. J. Appl. Phys. 113, 23102 (2013). 2-26. Pimenta, M. A. et al. Studying disorder in graphite-based systems by Raman spectroscopy. Phys. Chem. Chem. Phys. 9, 1276-1290 (2007).

2-27. Dallas, T., Holtz, M., Ahn, H. \& Downer, M. C. Structural phase of femtosecond-lasermelted graphite. Phys. Rev. B 49, 796-801 (1994).

2-28. Reich, S. \& Thomsen, C. Raman spectroscopy of graphite. Philos. Trans. R. Soc. Lond. Math. Phys. Eng. Sci. 362, 2271-2288 (2004).

2-29. Tuinstra, F. \& Koenig, J. L. Raman Spectrum of Graphite. J. Chem. Phys. 53, 1126-1130 (1970).

2-30. Zhang, Y. et al. Direct imprinting of microcircuits on graphene oxides film by femtosecond laser reduction. Nano Today 5, 15-20 (2010).

2-31. Cancado, L. G. et al. General equation for the determination of the crystallite size La of nanographite by Raman spectroscopy. Appl. Phys. Lett. 88, 163106-163106 (2006).

2-32. Deng, S. et al. Chemical forms of the fluorine, chlorine, oxygen and carbon in coal fly ash and their correlations with mercury retention. J. Hazard. Mater. 301, 400-406 (2016).

2-33. Wang, Z. et al. Functionalized N-doped interconnected carbon nanofibers as an anode material for sodium-ion storage with excellent performance. Carbon 55, 328-334 (2013).

2-34. Marcu, A. et al. Spatially resolved nanostructural transformation in graphite under femtosecond laser irradiation. Appl. Surf. Sci. 355, 477-483 (2015). 
2-35. Ranella, A., Barberoglou, M., Bakogianni, S., Fotakis, C. \& Stratakis, E. Tuning cell adhesion by controlling the roughness and wettability of 3D micro/nano silicon structures. Acta Biomater. 6, 2711-2720 (2010).

2-36. Dolatshahi-Pirouz, A. et al. Cell shape and spreading of stromal (mesenchymal) stem cells cultured on fibronectin coated gold and hydroxyapatite surfaces. Colloids Surf. B Biointerfaces $\mathbf{8 4}$, $18-25$ (2011).

2-37. Tojkander, S., Gateva, G. \& Lappalainen, P. Actin stress fibers-assembly, dynamics and biological roles. J. Cell Sci. 125, 1855-1864 (2012).

2-38. Gregory, T. R. Genome size evolution in animals. Evol. Genome 1, 4-87 (2005).

2-39. Webster, M., Witkin, K. L. \& Cohen-Fix, O. Sizing up the nucleus: nuclear shape, size and nuclear-envelope assembly. J. Cell Sci. 122, 1477-1486 (2009).

2-40. Walters, A. D., Bommakanti, A. \& Cohen-Fix, O. Shaping the nucleus: factors and forces. J. Cell. Biochem. 113, 2813-2821 (2012).

2-41. Gamaly, E. G. The physics of ultra-short laser interaction with solids at non-relativistic intensities. Phys. Rep. (2011).

2-42. Perez, D. \& Lewis, L. J. Ablation of Solids under Femtosecond Laser Pulses. Phys. Rev. Lett. 89, 255504 (2002).

2-43. Bourquard, F., Tite, T., Loir, A.-S., Donnet, C. \& Garrelie, F. Control of the Graphite Femtosecond Ablation Plume Kinetics by Temporal Laser Pulse Shaping: Effects on Pulsed Laser Deposition of Diamond-Like Carbon. J. Phys. Chem. C 118, 4377-4385 (2014).

2-44. Ionin, A. A. et al. Ultrafast femtosecond laser ablation of graphite. Laser Phys. Lett. 12, 75301 (2015). 
2-45. Yang, G. W. Laser ablation in liquids: applications in the synthesis of nanocrystals. Prog. Mater. Sci. 52, 648-698 (2007).

2-46. Lenardi, C. et al. Adhesion and Proliferation of Fibroblasts on Cluster-Assembled Nanostructured Carbon Films: The Role of Surface Morphology. J. Nanosci. Nanotechnol. 6, 3718-3730 (2006).

2-47. Tavangar, A., Premnath, P., Tan, B. \& Venkatakrishnan, K. Noble Hybrid Nanostructures as Efficient Anti-Proliferative Platforms for Human Breast Cancer Cell. ACS Appl. Mater. Interfaces 8, 10253-10265 (2016).

2-48. Bačáková, L., Filova, E., Rypáček, F., Švorčík, V. \& Starỳ, V. Cell adhesion on artificial materials for tissue engineering. Physiol Res 53, S35-S45 (2004).

2-49. Bacakova, L. et al. Carbon nanoparticles as substrates for cell adhesion and growth. Nanoparticles New Res. 39-107 (2008).

2-50. Woo, K. M., Chen, V. J. \& Ma, P. X. Nano-fibrous scaffolding architecture selectively enhances protein adsorption contributing to cell attachment. J. Biomed. Mater. Res. A 67A, 531537 (2003).

2-51. Nel, A. E. et al. Understanding biophysicochemical interactions at the nano-bio interface. Nat. Mater. 8, 543-557 (2009).

2-52. Tavangar, A., Tan, B. \& Venkatakrishnan, K. The Influence of Laser-Induced 3-D Titania Nanofibrous Platforms on Cell Behavior. J. Biomed. Nanotechnol. 9, 1837-1846 (2013). 2-53. Sanchez, V. C., Jachak, A., Hurt, R. H. \& Kane, A. B. Biological interactions of graphenefamily nanomaterials: an interdisciplinary review. Chem. Res. Toxicol. 25, 15-34 (2011). 
3-1. Li, R.; Wu, R.; Zhao, L.; Hu, Z.; Guo, S.; Pan, X.; Zou, H. Folate and Iron Difunctionalized Multiwall Carbon Nanotubes as Dual- Targeted Drug Nanocarrier to Cancer Cells. Carbon 2011, 49, 1797-1805.

3-2. Stancu, E. C.; Stanciuc, A.-M.; Vizireanu, S.; Luculescu, C.; Moldovan, L.; Achour, A.; Dinescu, G. Plasma Functionalization of Carbon Nanowalls and Its Effect on Attachment of Fibroblast-Like Cells. J. Phys. D: Appl. Phys. 2014, 47, 265203.

3-3. Lee, W. C.; Lim, C. H. Y. X.; Shi, H.; Tang, L. A. L.; Wang, Y.; Lim, C. T.; Loh, K. P. Origin of Enhanced Stem Cell Growth and Differentiation on Graphene and Graphene Oxide. ACS Nano $2011,5,7334-7341$.

3-4. Talukdar, Y.; Rashkow, J. T.; Lalwani, G.; Kanakia, S.; Sitharaman, B. The Effects of Graphene Nanostructures on Mesenchymal Stem Cells. Biomaterials 2014, 35, 4863-4877.

3-5. Feng, L.; Wu, L.; Wang, J.; Ren, J.; Miyoshi, D.; Sugimoto, N.; Qu, X. Detection of a Prognostic Indicator in Early-Stage Cancer Using Functionalized Graphene-Based Peptide Sensors. Adv. Mater.2012, 24, 125-131.

3-6. Qin, X. C.; Guo, Z. Y.; Liu, Z. M.; Zhang, W.; Wan, M. M.; Yang, B. W. Folic AcidConjugated Graphene Oxide for Cancer Targeted Chemo-Photothermal Therapy. J. Photochem. Photobiol., B 2013, 120, 156-162.

3-7. Jung, H. S.; Lee, M.-Y.; Kong, W. H.; Do, I. H.; Hahn, S. K. Nano Graphene Oxide-hyaluronic Acid Conjugate for Target Specific Cancer Drug Delivery. RSC Adv. 2014, 4, 14197.

3-8. De La Zerda, A.; Zavaleta, C.; Keren, S.; Vaithilingam, S.; Bodapati, S.; Liu, Z.; Levi, J.; Smith, B. R.; Ma, T.-J.; Oralkan, O.; Cheng, Z.; Chen, X.; Dai, H.; Khuri-Yakub, B. T.; Gambhir, 
S. S. Carbon Nanotubes as Photoacoustic Molecular Imaging Agents in Living Mice. Nat. Nanotechnol. 2008, 3, 557-562.

3-9. Watanabe, H.; Kondo, H.; Okamoto, Y.; Hiramatsu, M.; Sekine, M.; Baba, Y.; Hori, M. Carbon Nanowall Scaffold to Control Culturing of Cervical Cancer Cells. Appl. Phys. Lett. 2014, $105,244105$.

3-10. Ion, R.; Vizireanu, S.; Stancu, C. E.; Luculescu, C.; Cimpean, A.; Dinescu, G. Surface Plasma Functionalization Influences Macrophage Behavior on Carbon Nanowalls. Mater. Sci. Eng., C 2015, 48, 118-125.

3-11. Jesion, I.; Skibniewski, M.; Skibniewska, E.; Strupiński, W.; Szulc-Dąbrowska, L.; Krajewska, A.; Pasternak, I.; Kowalczyk, P.; Pińkowski, R. Graphene and Carbon Nanocompounds: Biofunctionalization and Applications in Tissue Engineering. Biotechnol. Biotechnol. Equip. 2015, 29, 415-422.

3-12. Zhang, X.; Wang, X.; Lu, Q.; Fu, C. Influence of Carbon Nanotube Scaffolds on Human Cervical Carcinoma HeLa Cell Viability and Focal Adhesion Kinase Expression. Carbon 2008, $46,453-460$.

3-13. Ryoo, S.-R.; Kim, Y.-K.; Kim, M.-H.; Min, D.-H. Behaviors of NIH-3T3 Fibroblasts on Graphene/Carbon Nanotubes: Proliferation, Focal Adhesion, and Gene Transfection Studies. ACS Nano 2010, 4, 6587-6598.

3-14. Aryaei, A.; Jayatissa, A. H.; Jayasuriya, A. C. The Effect of Graphene Substrate on Osteoblast Cell Adhesion and Proliferation. J. Biomed. Mater. Res., Part A 2014, 102, 3282-3290. 3-15. Zhang, Y.; Ali, S. F.; Dervishi, E.; Xu, Y.; Li, Z.; Casciano, D.; Biris, A. S. Cytotoxicity Effects of Graphene and Single-Wall Carbon Nanotubes in Neural Phaeochromocytoma-Derived PC12 Cells. ACS Nano 2010, 4, 3181-3186. 
3-16. Goenka, S.; Sant, V.; Sant, S. Graphene-Based Nanomaterials for Drug Delivery and Tissue Engineering. J. Controlled Release 2014, 173,75-88.

3-17. Kenry; Chaudhuri, P. K.; Loh, K. P.; Lim, C. T. Selective Accelerated Proliferation of Malignant Breast Cancer Cells on Planar Graphene Oxide Films. ACS Nano 2016, 10, 3424-3434.

3-18. Chowdhury, S. M.; Lalwani, G.; Zhang, K.; Yang, J. Y.; Neville, K.; Sitharaman, B. Cell Specific Cytotoxicity and Uptake of Graphene Nanoribbons. Biomaterials 2013, 34, 283-293.

3-19. Zhou, H.; Zhang, B.; Zheng, J.; Yu, M.; Zhou, T.; Zhao, K.; Jia, Y.; Gao, X.; Chen, C.; Wei, T. The Inhibition of Migration and Invasion of Cancer Cells by Graphene via the Impairment of Mitochondrial Respiration. Biomaterials 2014, 35, 1597-1607.

3-20. Markovic, Z. M.; Harhaji-Trajkovic, L. M.; Todorovic-Markovic, B. M.; Kepić, D. P.; Arsikin, K. M.; Jovanović, S. P.; Pantovic, A. C.; Dramićanin, M. D.; Trajkovic, V. S. In Vitro Comparison of the Photothermal Anticancer Activity of Graphene Nanoparticles and Carbon Nanotubes. Biomaterials 2011, 32, 1121-1129.

3-21. Sengupta, R.; Bhattacharya, M.; Bandyopadhyay, S.; Bhowmick, A. K. A Review on the Mechanical and Electrical Properties of Graphite and Modified Graphite Reinforced Polymer Composites. Prog. Polym. Sci. 2011, 36, 638-670.

3-22. Throckmorton, J.; Palmese, G. Direct Preparation of Few Layer Graphene Epoxy Nanocomposites from Untreated Flake Graphite. ACS Appl. Mater. Interfaces 2015, 7, $14870-14877$.

3-23. Bai, R. G.; Muthoosamy, K.; Zhou, M.; Ashokkumar, M.; Huang, N. M.; Manickam, S. Sonochemical and Sustainable Synthesis of Graphene-Gold (G-Au) Nanocomposites for Enzymeless and Selective Electrochemical Detection of Nitric Oxide. Biosens. Bioelectron. 2017, $87,622-629$. 
3-24. Bourquard, F.; Tite, T.; Loir, A.-S.; Donnet, C.; Garrelie, F. Control of the Graphite Femtosecond Ablation Plume Kinetics by Temporal Laser Pulse Shaping: Effects on Pulsed Laser Deposition of Diamond-Like Carbon. J. Phys. Chem. C 2014, 118, 4377-4385.

3-25. Tavangar, A.; Tan, B.; Venkatakrishnan, K. Study of the Formation of 3-D Titania Nanofibrous Structure by MHz Femtosecond Laser in Ambient Air. J. Appl. Phys. 2013, 113, 23102.

3-26. Tan, B.; Venkatakrishnan, K. Synthesis of Fibrous Nanoparticle Aggregates by Femtosecond Laser Ablation in Air. Opt. Express 2009, 17, 1064.

3-27. Wal, R. L. V.; Bryg, V. M.; Hays, M. D. XPS Analysis of Combustion Aerosols for Chemical Composition, Surface Chemistry, and Carbon Chemical State. Anal. Chem. 2011, 83, 1924-1930. 3-28. Estrade-Szwarckopf, H. XPS Photoemission in Carbonaceous Materials: A "defect" Peak beside the Graphitic Asymmetric Peak. Carbon 2004, 42, 1713-1721.

3-29. Hontoria-Lucas, C.; López-Peinado, A. J.; López-González, J. de D.; Rojas-Cervantes, M. L.; Martín-Aranda, R. M. Study of Oxygen-Containing Groups in a Series of Graphite Oxides: Physical and Chemical Characterization. Carbon 1995, 33, 1585-1592.

3-30. Tuinstra, F.; Koenig, J. L. Raman Spectrum of Graphite. J. Chem. Phys. 1970, 53, 1126-1130. (31) Ferrari, A. C.; Robertson, J. Interpretation of Raman Spectra of Disordered and Amorphous Carbon. Phys. Rev. B: Condens. Matter Mater. Phys. 2000, 61, 14095-14107.

3-32. Pimenta, M. A.; Dresselhaus, G.; Dresselhaus, M. S.; Cançado, L. G.; Jorio, A.; Saito, R. Studying Disorder in Graphite-Based Systems by Raman Spectroscopy. Phys. Chem. Chem. Phys. 2007, 9, 1276-1290.

3-33. Reich, S.; Thomsen, C. Raman Spectroscopy of Graphite. Philos. Trans. R. Soc., A 2004, 362, 2271-2288. 
3-34. Cançado, L. G.; Takai, K.; Enoki, T.; Endo, M.; Kim, Y. A.; Mizusaki, H.; Jorio, A.; Coelho, L. N.; Magalhães-Paniago, R.; Pimenta, M. A. General Equation for the Determination of the Crystallite Size La of Nanographite by Raman Spectroscopy. Appl. Phys. Lett. 2006, 88, 163106.

3-35. Mahmood, A. S.; Sivakumar, M.; Venkatakrishnan, K.; Tan, B. Enhancement in Optical Absorption of Silicon Fibrous Nanostructure Produced Using Femtosecond Laser Ablation. Appl. Phys. Lett. 2009, 95, 034107.

3-36. Tojkander, S.; Gateva, G.; Lappalainen, P. Actin Stress Fibers-Assembly, Dynamics and Biological Roles. J. Cell Sci. 2012, 125, 1855-1864.

3-37. Dolatshahi-Pirouz, A.; Jensen, T. H. L.; Kolind, K.; Bünger, C.; Kassem, M.; Foss, M.; Besenbacher, F. Cell Shape and Spreading of Stromal (Mesenchymal) Stem Cells Cultured on Fibronectin Coated Gold and Hydroxyapatite Surfaces. Colloids Surf., B 2011, 84, 18-25.

3-38. McBeath, R.; Pirone, D. M.; Nelson, C. M.; Bhadriraju, K.; Chen, C. S. Cell Shape, Cytoskeletal Tension, and RhoA Regulate Stem Cell Lineage Commitment. Dev. Cell 2004, 6, 483-495.

3-39. Santos, P. A.; Rocha, C. S.; Baptista, M. S. Adhesion and Proliferation of HeLa and Fibroblast Cells on Chemically-Modified Gold Surfaces. Colloids Surf., B 2014, 123, 429-438. 3-40. Hong, Y.; Fan, H.; Zhang, X. Synthesis and Protein Adsorption of Hierarchical Nanoporous Ultrathin Fibers. J. Phys. Chem. B 2009,113, 5837-5842.

3-41. Lamour, G.; Eftekhari-Bafrooei, A.; Borguet, E.; Souès, S.; Hamraoui, A. Neuronal Adhesion and Differentiation Driven by Nanoscale Surface Free-Energy Gradients. Biomaterials $2010,31,3762-3771$. 
3-42. Kunzmann, A.; Andersson, B.; Thurnherr, T.; Krug, H.; Scheynius, A.; Fadeel, B. Toxicology of Engineered Nanomaterials: Focus on Biocompatibility, Biodistribution and Biodegradation. Biochim. Biophys. Acta, Gen. Subj. 2011, 1810, 361-373.

3-43. Lesniak, A.; Salvati, A.; Santos-Martinez, M. J.; Radomski, M. W.; Dawson, K. A.; Åberg, C. Nanoparticle Adhesion to the Cell Membrane and Its Effect on Nanoparticle Uptake Efficiency. J. Am.Chem. Soc. 2013, 135, 1438-1444.

3-44. Dykman, L. A.; Khlebtsov, N. G. Uptake of Engineered Gold Nanoparticles into Mammalian Cells. Chem. Rev. 2014, 114, 1258-1288.

3-45. Zhu, M.; Nie, G.; Meng, H.; Xia, T.; Nel, A.; Zhao, Y. Physicochemical Properties Determine Nanomaterial Cellular Uptake, Transport, and Fate. Acc. Chem. Res. 2013, 46, $622-631$.

3-46. Zhang, S.; Gao, H.; Bao, G. Physical Principles of Nanoparticle Cellular Endocytosis. ACS Nano 2015, 9, 8655-8671.

3-47. Dalal, C.; Saha, A.; Jana, N. R. Nanoparticle Multivalency Directed Shifting of Cellular Uptake Mechanism. J. Phys. Chem. C 2016, 120, 6778-6786.

3-48. Slowing, I.; Trewyn, B. G.; Lin, V. S.-Y. Effect of Surface Functionalization of MCM-41Type Mesoporous Silica Nanoparticles on the Endocytosis by Human Cancer Cells. J. Am. Chem. Soc. 2006, 128, 14792-14793.

3-49. Villanueva, A.; de la Presa, P.; Alonso, J. M.; Rueda, T.; Martínez, A.; Crespo, P.; Morales, M. P.; Gonzalez-Fernandez, M. A.; Valdés, J.; Rivero, G. Hyperthermia HeLa Cell Treatment with Silica-Coated Manganese Oxide Nanoparticles. J. Phys. Chem. C 2010, 114, 1976-1981.

3-50. Solon, J.; Levental, I.; Sengupta, K.; Georges, P. C.; Janmey, P. A. Fibroblast Adaptation and Stiffness Matching to Soft Elastic Substrates. Biophys. J. 2007, 93, 4453-4461. 
3-51. Kim, K. S.; Cho, C. H.; Park, E. K.; Jung, M.-H.; Yoon, K.-S.; Park, H.-K. AFM-Detected Apoptotic Changes in Morphology and Biophysical Property Caused by Paclitaxel in Ishikawa and HeLa Cells. PLoS One 2012, 7, No. e30066.

3-52. Albanese, A.; Tang, P. S.; Chan, W. C. W. The Effect of Nanoparticle Size, Shape, and Surface Chemistry on Biological Systems. Annu. Rev. Biomed. Eng. 2012, 14, 1-16.

3-53. Perez, D.; Lewis, L. J. Ablation of Solids under Femtosecond Laser Pulses. Phys. Rev. Lett. $2002,89,255504$.

3-54. Bourquard, F.; Tite, T.; Loir, A.-S.; Donnet, C.; Garrelie, F.Control of the Graphite Femtosecond Ablation Plume Kinetics by Temporal Laser Pulse Shaping: Effects on Pulsed Laser Deposition of Diamond-Like Carbon. J. Phys. Chem. C 2014, 118, 4377-4385.

3-55. Łopacińska, J. M.; Grădinaru, C.; Wierzbicki, R.; Købler, C.; Schmidt, M. S.; Madsen, M. T.; Skolimowski, M.; Dufva, M.; Flyvbjerg, H.; Mølhave, K. Cell Motility, Morphology, Viability and Proliferation in Response to Nanotopography on Silicon Black. Nanoscale 2012, 4, 3739-3745.

3-56. Zhang, F.; Jiang, Y.; Liu, X.; Meng, J.; Zhang, P.; Liu, H.; Yang, G.; Li, G.; Jiang, L.; Wan, L.-J.; Hu, J.-S.; Wang, S. Hierarchical Nanowire Arrays as Three-Dimensional Fractal Nanobiointerfaces for High Efficient Capture of Cancer Cells. Nano Lett. 2016, 16, 766-772.

3-57. Chen, W.; Weng, S.; Zhang, F.; Allen, S.; Li, X.; Bao, L.; Lam, R. H. W.; Macoska, J. A.; Merajver, S. D.; Fu, J. Nanoroughened Surfaces for Efficient Capture of Circulating Tumor Cells without Using Capture Antibodies. ACS Nano 2013, 7, 566-575.

3-58. Kalbacova, M.; Broz, A.; Kalbac, M. Influence of the Fetal Bovine Serum Proteins on the Growth of Human Osteoblast Cells on Graphene. J. Biomed. Mater. Res., Part A 2012, 100, 3001-3007. 
3-59. Nel, A. E.; Mädler, L.; Velegol, D.; Xia, T.; Hoek, E. M. V.; Somasundaran, P.; Klaessig, F.; Castranova, V.; Thompson, M. Understanding Biophysicochemical Interactions at the Nano-bio

3-60. Interface. Nat. Mater. 2009, 8, 543-557. (60) Hynes, R. O. Integrins: Bidirectional, Allosteric Signaling Machines. Cell 2002, 110, 673-687.

3-61. Arima, Y.; Iwata, H. Effect of Wettability and Surface Functional Groups on Protein Adsorption and Cell Adhesion Using Well-Defined Mixed Self-Assembled Monolayers. Biomaterials 2007, 28, 3074-3082.

3-62. Tavangar, A.; Premnath, P.; Tan, B.; Venkatakrishnan, K. Noble Hybrid Nanostructures as Efficient Anti-Proliferative Platforms for Human Breast Cancer Cell. ACS Appl. Mater. Interfaces $2016,8,10253-10265$.

3-63. Bacakova, L.; Grausova, L.; Vandrovcova, M.; Vacik, J.; Frazcek, A.; Blazewicz, S.; Kromka, A.; Rezek, B.; Vanecek, M.; Nesladek, M.; et al. Carbon Nanoparticles as Substrates for Cell Adhesion and Growth. In Nanoparticles: New Research; Nova Science, 2008; pp 39-107.

3-64. Woo, K. M.; Chen, V. J.; Ma, P. X. Nano-Fibrous Scaffolding Architecture Selectively Enhances Protein Adsorption Contributing to Cell Attachment. J. Biomed. Mater. Res., Part A 2003, 67, 531-537.

3-65. Humphries, J. D.; Wang, P.; Streuli, C.; Geiger, B.; Humphries, M. J.; Ballestrem, C. Vinculin Controls Focal Adhesion Formation by Direct Interactions with Talin and Actin. J. Cell Biol. 2007, 179, 1043-1057.

3-66. Benjamin, S.; Sheyn, D.; Ben-David, S.; Oh, A.; Kallai, I.; Li, N.; Gazit, D.; Gazit, Z. Oxygenated Environment Enhances Both Stem Cell Survival and Osteogenic Differentiation. Tissue Eng., Part A 2013, 19, 748-758. 
3-67. Wang, K.; Ruan, J.; Song, H.; Zhang, J.; Wo, Y.; Guo, S.; Cui, D. Biocompatibility of Graphene Oxide. Nanoscale Res. Lett 2010, 6, 8.

3-68. Gollavelli, G.; Ling, Y.-C. Multi-Functional Graphene as an in Vitro and in Vivo Imaging Probe. Biomaterials 2012, 33, 2532-2545.

3-69. Liao, K.-H.; Lin, Y.-S.; Macosko, C. W.; Haynes, C. L. Cytotoxicity of Graphene Oxide and Graphene in Human Erythrocytes and Skin Fibroblasts. ACS Appl. Mater. Interfaces 2011, 3, $2607-2615$.

4-1. Hong, G.; Diao, S.; Antaris, A. L.; Dai, H. Carbon Nanomaterials for Biological Imaging and Nanomedicinal Therapy. Chem. Rev. 2015, 115 (19), 10816-10906.

4-2. Bartelmess, J.; Quinn, S. J.; Giordani, S. Carbon Nanomaterials: Multi-Functional Agents for Biomedical Fluorescence and Raman Imaging. Chem Soc Rev 2015, 44 (14), 4672-4698.

4-3. Yagur-Kroll, S.; Schreuder, E.; Ingham, C. J.; Heideman, R.; Rosen, R.; Belkin, S. A Miniature Porous Aluminum Oxide-Based Flow-Cell for Online Water Quality Monitoring Using Bacterial Sensor Cells. Biosens. Bioelectron. 2015, 64 (Supplement C), 625-632.

4-4. Selvakumar, R.; Aravindh, S.; Kaushik, C.; Katarani, V.; Thorat, V.; Gireesan, P.; Jayavignesh, V.; Swaminathan, K.; Raj, K. Screening of Silver Nanoparticles Containing Carbonized Yeast Cells for Adsorption of Few Long-Lived Active Radionuclides. J. Radioanal. Nucl. Chem. 2011, 288 (2), 629-633.

4-5. Lauterbach, T.; Manna, M.; Ruhnow, M.; Wisantoso, Y.; Wang, Y.; Matysik, A.; Oglęcka, K.; Mu, Y.; Geifman-Shochat, S.; Wohland, T.; Kraut, R. Weak Glycolipid Binding of a Microdomain-Tracer Peptide Correlates with Aggregation and Slow Diffusion on Cell Membranes. PLoS ONE 2012, 7 (12). 
4-6. Bagó, J. R.; Aguilar, E.; Alieva, M.; Soler-Botija, C.; Vila, O. F.; Claros, S.; Andrades, J. A.; Becerra, J.; Rubio, N.; Blanco, J. In Vivo Bioluminescence Imaging of Cell Differentiation in Biomaterials: A Platform for Scaffold Development. Tissue Eng. Part New Rochelle 2013, 19 (56), 593-603.

4-7. Antonio, K. A.; Schultz, Z. D. Advances in Biomedical Raman Microscopy. Anal. Chem. 2014, 86 (1), 30-46.

4-8. Matousek, P.; Stone, N. Recent Advances in the Development of Raman Spectroscopy for Deep Non-invasive Medical Diagnosis. J. Biophotonics 2013, 6 (1), 7-19.

4-9. Notingher, I.; Hench, L. L. Raman Microspectroscopy: A Noninvasive Tool for Studies of Individual Living Cells in Vitro. Expert Rev. Med. Devices 2006, 3 (2), 215-234.

4-10. Huang, Y.-S.; Karashima, T.; Yamamoto, M.; Hamaguchi, H. Molecular-Level Investigation of the Structure, Transformation, and Bioactivity of Single Living Fission Yeast Cells by Time- and Space-Resolved Raman Spectroscopy. Biochemistry (Mosc.) 2005, 44 (30), 10009-10019.

4-11. Notingher, I.; Verrier, S.; Haque, S.; Polak, J. M.; Hench, L. L. Spectroscopic Study of Human Lung Epithelial Cells (A549) in Culture: Living Cells versus Dead Cells. Biopolymers 2003, 72 (4), 230-240.

4-12. Mahadevan-Jansen, A.; Richards-Kortum, R. R. Raman Spectroscopy for the Detection of Cancers and Precancers. J. Biomed. Opt. 1996, 1 (1), 31.

4-13. Manikandan, M.; Abdelhamid, H. N.; Talib, A.; Wu, H.-F. Facile Synthesis of Gold Nanohexagons on Graphene Templates in Raman Spectroscopy for Biosensing Cancer and Cancer Stem Cells. Biosens. Bioelectron. 2014, 55, 180-186. 
4-14. Gopinath, A.; Boriskina, S. V.; Premasiri, W. R.; Ziegler, L.; Reinhard, B. M.; Dal Negro, L. Plasmonic Nanogalaxies: Multiscale Aperiodic Arrays for Surface-Enhanced Raman Sensing. Nano Lett. 2009, 9 (11), 3922-3929.

4-15. Maznichenko, D.; Venkatakrishnan, K.; Tan, B. Stimulating Multiple SERS Mechanisms by a Nanofibrous Three-Dimensional Network Structure of Titanium Dioxide (TiO2). J. Phys. Chem. C 2013, 117 (1), 578-583.

4-16. Colomban, P.; Tournié, A.; Maucuer, M.; Meynard, P. On-Site Raman and XRF Analysis of Japanese/Chinese Bronze/brass Patina - the Search for Specific Raman Signatures. J. Raman Spectrosc. 2012, 43 (6), 799-808.

4-17. Rodríguez-Lorenzo, L.; Krpetic, Z.; Barbosa, S.; Alvarez-Puebla, R. A.; Liz-Marzán, L. M.; Prior, I. A.; Brust, M. Intracellular Mapping with SERS-Encoded Gold Nanostars. Integr. Biol. 2011, 3 (9), 922-926.

4-18. Jain, P. K.; Huang, X.; El-Sayed, I. H.; El-Sayed, M. A. Review of Some Interesting Surface Plasmon Resonance-Enhanced Properties of Noble Metal Nanoparticles and Their Applications to Biosystems. Plasmonics 2007, 2 (3), 107-118.

4-19. Register, J.; Fales, A.; Wang, H.-N.; Norton, S.; Cho, E.; Boico, A.; Pradhan, S.; Kim, J.; Schroeder, T.; Wisniewski, N.; Klitzman, B.; Vo-Dinh, T. In Vivo Detection of SERS-Encoded Plasmonic Nanostars in Human Skin Grafts and Live Animal Models. Anal. Bioanal. Chem. 2015, 407 (27), 8215-8224.

4-20. Xu, W.; Ling, X.; Xiao, J.; Dresselhaus, M. S.; Kong, J.; Xu, H.; Liu, Z.; Zhang, J. Surface Enhanced Raman Spectroscopy on a Flat Graphene Surface. Proc. Natl. Acad. Sci. 2012, 109 (24), $9281-9286$. 
4-21. Zhang, Y.; Wang, C.; Wang, J.; Chen, L.; Li, J.; Liu, Y.; Zhao, X.; Wang, Y.; Yang, J. Nanocap Array of Au: Ag Composite for Surface-Enhanced Raman Scattering. Spectrochim. Acta. A. Mol. Biomol. Spectrosc. 2016, 152, 461-467.

4-22. Kang, Z.; Gu, Y.; Yan, X.; Bai, Z.; Liu, Y.; Liu, S.; Zhang, X.; Zhang, Z.; Zhang, X.; Zhang, Y. Enhanced Photoelectrochemical Property of ZnO Nanorods Array Synthesized on Reduced Graphene Oxide for Self-Powered Biosensing Application. Biosens. Bioelectron. 2015, 64, 499-504.

4-23. Li, W.; Zamani, R.; Rivera Gil, P.; Pelaz, B.; Ibáñez, M.; Cadavid, D.; Shavel, A.; AlvarezPuebla, R. A.; Parak, W. J.; Arbiol, J.; others. CuTe Nanocrystals: Shape and Size Control, Plasmonic Properties, and Use as SERS Probes and Photothermal Agents. J. Am. Chem. Soc. 2013, 135 (19), 7098-7101.

4-24. Mao, Z.; Song, W.; Chen, L.; Ji, W.; Xue, X.; Ruan, W.; Li, Z.; Mao, H.; Ma, S.; Lombardi, J. R.; others. Metal-semiconductor Contacts Induce the Charge-Transfer Mechanism of SurfaceEnhanced Raman Scattering. J. Phys. Chem. C 2011, 115 (37), 18378-18383.

4-25. Wang, X.; Shi, W.; She, G.; Mu, L. Surface-Enhanced Raman Scattering (SERS) on Transition Metal and Semiconductor Nanostructures. Phys. Chem. Chem. Phys. 2012, 14 (17), $5891-5901$.

4-26. Batra, B.; Lata, S.; Pundir, C. Construction of an Improved Amperometric Acrylamide Biosensor Based on Hemoglobin Immobilized onto Carboxylated Multi-Walled Carbon Nanotubes/iron Oxide Nanoparticles/chitosan Composite Film. Bioprocess Biosyst. Eng. 2013, 36 (11), 1591-1599.

4-27. Shan, C.; Yang, H.; Han, D.; Zhang, Q.; Ivaska, A.; Niu, L. Graphene/AuNPs/chitosan Nanocomposites Film for Glucose Biosensing. Biosens. Bioelectron. 2010, 25 (5), 1070-1074. 
4-28. Kailashiya, J.; Singh, N.; Singh, S. K.; Agrawal, V.; Dash, D. Graphene Oxide-Based Biosensor for Detection of Platelet-Derived Microparticles: A Potential Tool for Thrombus Risk Identification. Biosens. Bioelectron. 2015, 65, 274-280.

4-29. Kumar, S.; Ahlawat, W.; Kumar, R.; Dilbaghi, N. Graphene, Carbon Nanotubes, Zinc Oxide and Gold as Elite Nanomaterials for Fabrication of Biosensors for Healthcare. Biosens. Bioelectron. 2015, 70, 498-503.

4-30. Liu, Y.; Zhao, Y.; Sun, B.; Chen, C. Understanding the Toxicity of Carbon Nanotubes. Acc. Chem. Res. 2013, 46 (3), 702-713.

4-31. Sun, Y.; Liu, K.; Miao, J.; Wang, Z.; Tian, B.; Zhang, L.; Li, Q.; Fan, S.; Jiang, K. Highly Sensitive Surface-Enhanced Raman Scattering Substrate Made from Superaligned Carbon Nanotubes. Nano Lett. 2010, 10 (5), 1747-1753.

4-32. Lee, S.; Hahm, M. G.; Vajtai, R.; Hashim, D. P.; Thurakitseree, T.; Chipara, A. C.; Ajayan, P. M.; Hafner, J. H. Utilizing 3D SERS Active Volumes in Aligned Carbon Nanotube Scaffold Substrates. Adv. Mater. 2012, 24 (38), 5261-5266.

4-33. Bhirde, A. A.; Liu, G.; Jin, A.; Iglesias-Bartolome, R.; Sousa, A. A.; Leapman, R. D.; Gutkind, J. S.; Lee, S.; Chen, X. Combining Portable Raman Probes with Nanotubes for Theranostic Applications. Theranostics 2011, 1, 310.

4-34. Alwarappan, S.; Erdem, A.; Liu, C.; Li, C.-Z. Probing the Electrochemical Properties of Graphene Nanosheets for Biosensing Applications. J. Phys. Chem. C 2009, 113 (20), 8853-8857. 4-35. He, S.; Liu, K.-K.; Su, S.; Yan, J.; Mao, X.; Wang, D.; He, Y.; Li, L.-J.; Song, S.; Fan, C. Graphene-Based High-Efficiency Surface-Enhanced Raman Scattering-Active Platform for Sensitive and Multiplex DNA Detection. Anal. Chem. 2012, 84 (10), 4622-4627. 
4-36. Basu, P. K.; Indukuri, D.; Keshavan, S.; Navratna, V.; Vanjari, S. R. K.; Raghavan, S.; Bhat, N. Graphene Based E. Coli Sensor on Flexible Acetate Sheet. Sens. Actuators B Chem. 2014, $190,342-347$.

4-37. Tiwari, I.; Singh, M.; Pandey, C. M.; Sumana, G. Electrochemical Genosensor Based on Graphene Oxide Modified Iron Oxide-chitosan Hybrid Nanocomposite for Pathogen Detection. Sens. Actuators B Chem. 2015, 206, 276-283.

4-38. Huang, H.; Bai, W.; Dong, C.; Guo, R.; Liu, Z. An Ultrasensitive Electrochemical DNA Biosensor Based on graphene/Au Nanorod/polythionine for Human Papillomavirus DNA Detection. Biosens. Bioelectron. 2015, 68, 442-446.

4-39. Chowdhury, A. R. H.; Tavangar, A.; Tan, B.; Venkatakrishnan, K. Biofunctionalized 3-D Carbon Nano-Network Platform for Enhanced Fibroblast Cell Adhesion. Sci. Rep. 2017, 7.

4-40. Ling, X.; Xie, L.; Fang, Y.; Xu, H.; Zhang, H.; Kong, J.; Dresselhaus, M. S.; Zhang, J.; Liu, Z. Can Graphene Be Used as a Substrate for Raman Enhancement? Nano Lett. 2009, 10 (2), $553-561$.

4-41. Bourquard, F.; Tite, T.; Loir, A.-S.; Donnet, C.; Garrelie, F. Control of the Graphite Femtosecond Ablation Plume Kinetics by Temporal Laser Pulse Shaping: Effects on Pulsed Laser Deposition of Diamond-Like Carbon. J. Phys. Chem. C 2014, 118 (8), 4377-4385.

4-42. Tavangar, A.; Tan, B.; Venkatakrishnan, K. Study of the Formation of 3-D Titania Nanofibrous Structure by MHz Femtosecond Laser in Ambient Air. J. Appl. Phys. 2013, 113 (2), 23102.

4-43. Tan, B.; Venkatakrishnan, K. Synthesis of Fibrous Nanoparticle Aggregates by Femtosecond Laser Ablation in Air. Opt. Express 2009, 17 (2), 1064. 
4-44. Estrade-Szwarckopf, H. XPS Photoemission in Carbonaceous Materials: A “defect” Peak beside the Graphitic Asymmetric Peak. Carbon 2004, 42 (8-9), 1713-1721.

4-45. Hontoria-Lucas, C.; López-Peinado, A. J.; López-González, J. de D.; Rojas-Cervantes, M. L.; Martín-Aranda, R. M. Study of Oxygen-Containing Groups in a Series of Graphite Oxides: Physical and Chemical Characterization. Carbon 1995, 33 (11), 1585-1592.

4-46. Tuinstra, F.; Koenig, J. L. Raman Spectrum of Graphite. J. Chem. Phys. 1970, 53 (3), $1126-1130$.

4-47. Ferrari, A. C.; Robertson, J. Interpretation of Raman Spectra of Disordered and Amorphous Carbon. Phys. Rev. B 2000, 61 (20), 14095-14107.

4-48. Reich, S.; Thomsen, C. Raman Spectroscopy of Graphite. Philos. Trans. R. Soc. Lond. Math. Phys. Eng. Sci. 2004, 362 (1824), 2271-2288.

4-49. Aroca, R. Surface-Enhanced Vibrational Spectroscopy; John Wiley \& Sons, 2006.

4-50. Schatz, G.; Young, M.; Van Duyne, R. Electromagnetic Mechanism of SERS. Surf.Enhanc. Raman Scatt. 2006, 19-45.

4-51. Le Ru, E.; Etchegoin, P. Principles of Surface-Enhanced Raman Spectroscopy: And Related Plasmonic Effects; Elsevier, 2008.

4-52. Persson, B. N. J.; Zhao, K.; Zhang, Z. Chemical Contribution to Surface-Enhanced Raman Scattering. Phys. Rev. Lett. 2006, 96 (20), 207401.

4-53. Otto, A. The "chemical” (Electronic) Contribution to Surface-enhanced Raman Scattering. J. Raman Spectrosc. 2005, 36 (6-7), 497-509.

4-54. Lu, Y. H.; Chen, W.; Feng, Y. P.; He, P. M. Tuning the Electronic Structure of Graphene by an Organic Molecule. J. Phys. Chem. B 2009, 113 (1), 2-5. 
4-55. Ling, X.; Fang, W.; Lee, Y.-H.; Araujo, P. T.; Zhang, X.; Rodriguez-Nieva, J. F.; Lin, Y.; Zhang, J.; Kong, J.; Dresselhaus, M. S. Raman Enhancement Effect on Two-Dimensional Layered Materials: Graphene, H-BN and MoS2. Nano Lett. 2014, 14 (6), 3033-3040.

4-56. Powell, J. A.; Venkatakrishnan, K.; Tan, B. Hybridized Enhancement of the SERS Detection of Chemical and Bio-Marker Molecules through Au Nanosphere Ornamentation of Hybrid Amorphous/crystalline Si Nanoweb Nanostructure Biochip Devices. J. Mater. Chem. B 2016, 4 (34), 5713-5728.

4-57. Kneipp, J. Nanosensors Based on SERS for Applications in Living Cells. Surf.-Enhanc. Raman Scatt. 2006, 335-349.

4-58. Santos, P. A.; Rocha, C. S.; Baptista, M. S. Adhesion and Proliferation of HeLa and Fibroblast Cells on Chemically-Modified Gold Surfaces. Colloids Surf. B Biointerfaces 2014, 123, $429-438$.

4-59. Hong, Y.; Fan, H.; Zhang, X. Synthesis and Protein Adsorption of Hierarchical Nanoporous Ultrathin Fibers. J. Phys. Chem. B 2009, 113 (17), 5837-5842.

4-60. Lamour, G.; Eftekhari-Bafrooei, A.; Borguet, E.; Souès, S.; Hamraoui, A. Neuronal Adhesion and Differentiation Driven by Nanoscale Surface Free-Energy Gradients. Biomaterials 2010, 31 (14), 3762-3771.

4-61. Kunzmann, A.; Andersson, B.; Thurnherr, T.; Krug, H.; Scheynius, A.; Fadeel, B. Toxicology of Engineered Nanomaterials: Focus on Biocompatibility, Biodistribution and Biodegradation. Biochim. Biophys. Acta BBA - Gen. Subj. 2011, 1810 (3), 361-373.

4-62. Lesniak, A.; Salvati, A.; Santos-Martinez, M. J.; Radomski, M. W.; Dawson, K. A.; Åberg, C. Nanoparticle Adhesion to the Cell Membrane and Its Effect on Nanoparticle Uptake Efficiency. J. Am. Chem. Soc. 2013, 135 (4), 1438-1444. 
4-63. Dykman, L. A.; Khlebtsov, N. G. Uptake of Engineered Gold Nanoparticles into Mammalian Cells. Chem. Rev. 2014, 114 (2), 1258-1288.

4-64. Zhu, M.; Nie, G.; Meng, H.; Xia, T.; Nel, A.; Zhao, Y. Physicochemical Properties Determine Nanomaterial Cellular Uptake, Transport, and Fate. Acc. Chem. Res. 2013, 46 (3), 622631.

4-65. Ochsenkühn, M. A.; Jess, P. R. T.; Stoquert, H.; Dholakia, K.; Campbell, C. J. Nanoshells for Surface-Enhanced Raman Spectroscopy in Eukaryotic Cells: Cellular Response and Sensor Development. ACS Nano 2009, 3 (11), 3613-3621.

4-66. Movasaghi, Z.; Rehman, S.; Rehman, I. U. Raman Spectroscopy of Biological Tissues. Appl. Spectrosc. Rev. 2007, 42 (5), 493-541.

4-67. Short, K. W.; Carpenter, S.; Freyer, J. P.; Mourant, J. R. Raman Spectroscopy Detects Biochemical Changes Due to Proliferation in Mammalian Cell Cultures. Biophys. J. 2005, 88 (6), 4274-4288.

4-68. Dolatshahi-Pirouz, A.; Jensen, T. H. L.; Kolind, K.; Bünger, C.; Kassem, M.; Foss, M.; Besenbacher, F. Cell Shape and Spreading of Stromal (Mesenchymal) Stem Cells Cultured on Fibronectin Coated Gold and Hydroxyapatite Surfaces. Colloids Surf. B Biointerfaces 2011, 84 (1), 18-25.

4-69. McBeath, R.; Pirone, D. M.; Nelson, C. M.; Bhadriraju, K.; Chen, C. S. Cell Shape, Cytoskeletal Tension, and RhoA Regulate Stem Cell Lineage Commitment. Dev. Cell 2004, 6 (4), $483-495$.

4-70. Luo, S.-C.; Sivashanmugan, K.; Liao, J.-D.; Yao, C.-K.; Peng, H.-C. Nanofabricated SERS-Active Substrates for Single-Molecule to Virus Detection in Vitro: A Review. Biosens. Bioelectron. 2014, 61, 232-240. 
4-71. Perez, D.; Lewis, L. J. Ablation of Solids under Femtosecond Laser Pulses. Phys. Rev. Lett. 2002, 89 (25), 255504.

4-72. Roiter, Y.; Ornatska, M.; Rammohan, A. R.; Balakrishnan, J.; Heine, D. R.; Minko, S. Interaction of Nanoparticles with Lipid Membrane. Nano Lett. 2008, 8 (3), 941-944.

4-73. Kneipp, J.; Kneipp, H.; McLaughlin, M.; Brown, D.; Kneipp, K. In Vivo Molecular Probing of Cellular Compartments with Gold Nanoparticles and Nanoaggregates. Nano Lett. 2006, $6(10), 2225-2231$.

4-74. Shukla, R.; Bansal, V.; Chaudhary, M.; Basu, A.; Bhonde, R. R.; Sastry, M. Biocompatibility of Gold Nanoparticles and Their Endocytotic Fate inside the Cellular Compartment: A Microscopic Overview. Langmuir 2005, 21 (23), 10644-10654.

4-75. Chithrani, B. D.; Ghazani, A. A.; Chan, W. C. Determining the Size and Shape Dependence of Gold Nanoparticle Uptake into Mammalian Cells. Nano Lett. 2006, 6 (4), 662-668.

4-76. Chowdhury, A. R. H.; Tan, B.; Venkatakrishnan, K. Fibroblast-Cytophilic and HeLaCytotoxic Dual Function Carbon Nanoribbon-Network Platform. ACS Appl. Mater. Interfaces 2017.

4-77. Chaudhuri, P. K.; Loh, K. P.; Lim, C. T. Selective Accelerated Proliferation of Malignant Breast Cancer Cells on Planar Graphene Oxide Films. ACS Nano 2016, 10 (3), 3424-3434.

4-78. Benjamin, S.; Sheyn, D.; Ben-David, S.; Oh, A.; Kallai, I.; Li, N.; Gazit, D.; Gazit, Z. Oxygenated Environment Enhances Both Stem Cell Survival and Osteogenic Differentiation. Tissue Eng. Part A 2013, 19 (5-6), 748-758.

4-79. Gollavelli, G.; Ling, Y.-C. Multi-Functional Graphene as an in Vitro and in Vivo Imaging Probe. Biomaterials 2012, 33 (8), 2532-2545. 
5-1. $\quad$ Lyng, F. M.; Faolain, E.; Conroy, J.; Meade, A. D.; Knief, P.; Duffy, B.; Hunter, M. B.; Byrne, J. M.; Kelehan, P.; Byrne, H. J. Vibrational Spectroscopy for Cervical Cancer Pathology, from Biochemical Analysis to Diagnostic Tool. Exp. Mol. Pathol. 2007, 82 (2), 121-129.

5-2. Parkin, D. M.; Bray, F.; Ferlay, J.; Pisani, P. Global Cancer Statistics, 2002. CA. Cancer J. Clin. 2005, 55 (2), 74-108.

5-3. Siegel, R. L.; Miller, K. D.; Jemal, A. Cancer Statistics, 2015. CA. Cancer J. Clin. 2015, $65(1), 5-29$.

5-4. Wallace, G. Q.; Zuin, M. S.; Tabatabaei, M.; Gobbo, P.; Lagugné-Labarthet, F.; Workentin, M. S. Gold Nanosponges (AuNS): A Versatile Nanostructure for Surface-Enhanced Raman Spectroscopic Detection of Small Molecules and Biomolecules. Analyst 2015, 140 (21), 72787282.

5-5. Bizzarri, A. R.; Cannistraro, S. SERS Detection of Thrombin by Protein Recognition Using Functionalized Gold Nanoparticles. Nanomedicine Nanotechnol. Biol. Med. 2007, 3 (4), 306-310.

5-6. Convertino, A.; Mussi, V.; Maiolo, L. Disordered Array of Au Covered Silicon Nanowires for SERS Biosensing Combined with Electrochemical Detection. Sci. Rep. 2016, 6, 25099.

5-7. Wang, H.-N.; Fales, A. M.; Vo-Dinh, T. Plasmonics-Based SERS Nanobiosensor for Homogeneous Nucleic Acid Detection. Nanomedicine Nanotechnol. Biol. Med. 2015, 11 (4), 811814.

5-8. Mahadevan-Jansen, A.; Richards-Kortum, R. R. Raman Spectroscopy for the Detection of Cancers and Precancers. J. Biomed. Opt. 1996, 1 (1), 31.

5-9. Manikandan, M.; Abdelhamid, H. N.; Talib, A.; Wu, H.-F. Facile Synthesis of Gold Nanohexagons on Graphene Templates in Raman Spectroscopy for Biosensing Cancer and Cancer Stem Cells. Biosens. Bioelectron. 2014, 55, 180-186. 
5-10. Gopinath, A.; Boriskina, S. V.; Premasiri, W. R.; Ziegler, L.; Reinhard, B. M.; Dal Negro, L. Plasmonic Nanogalaxies: Multiscale Aperiodic Arrays for Surface-Enhanced Raman Sensing. Nano Lett. 2009, 9 (11), 3922-3929.

5-11. Maznichenko, D.; Venkatakrishnan, K.; Tan, B. Stimulating Multiple SERS Mechanisms by a Nanofibrous Three-Dimensional Network Structure of Titanium Dioxide (TiO2). J. Phys. Chem. C 2013, 117 (1), 578-583.

5-12. Colomban, P.; Tournié, A.; Maucuer, M.; Meynard, P. On-Site Raman and XRF Analysis of Japanese/Chinese Bronze/brass Patina - the Search for Specific Raman Signatures. J. Raman Spectrosc. 2012, 43 (6), 799-808.

5-13. Rodríguez-Lorenzo, L.; Krpetic, Z.; Barbosa, S.; Alvarez-Puebla, R. A.; Liz-Marzán, L. M.; Prior, I. A.; Brust, M. Intracellular Mapping with SERS-Encoded Gold Nanostars. Integr. Biol. 2011, 3 (9), 922-926.

5-14. Hong, G.; Diao, S.; Antaris, A. L.; Dai, H. Carbon Nanomaterials for Biological Imaging and Nanomedicinal Therapy. Chem. Rev. 2015, 115 (19), 10816-10906.

5-15. Bartelmess, J.; Quinn, S. J.; Giordani, S. Carbon Nanomaterials: Multi-Functional Agents for Biomedical Fluorescence and Raman Imaging. Chem Soc Rev 2015, 44 (14), 4672-4698.

5-16. Liu, Y.; Zhao, Y.; Sun, B.; Chen, C. Understanding the Toxicity of Carbon Nanotubes. Acc. Chem. Res. 2013, 46 (3), 702-713.

5-17. Kumar, S.; Ahlawat, W.; Kumar, R.; Dilbaghi, N. Graphene, Carbon Nanotubes, Zinc Oxide and Gold as Elite Nanomaterials for Fabrication of Biosensors for Healthcare. Biosens. Bioelectron. 2015, 70, 498-503. 
5-18. Sun, Y.; Liu, K.; Miao, J.; Wang, Z.; Tian, B.; Zhang, L.; Li, Q.; Fan, S.; Jiang, K. Highly

Sensitive Surface-Enhanced Raman Scattering Substrate Made from Superaligned Carbon Nanotubes. Nano Lett. 2010, 10 (5), 1747-1753.

5-19. Bhirde, A. A.; Liu, G.; Jin, A.; Iglesias-Bartolome, R.; Sousa, A. A.; Leapman, R. D.;

Gutkind, J. S.; Lee, S.; Chen, X. Combining Portable Raman Probes with Nanotubes for Theranostic Applications. Theranostics 2011, 1, 310.

5-20. Ling, X.; Xie, L.; Fang, Y.; Xu, H.; Zhang, H.; Kong, J.; Dresselhaus, M. S.; Zhang, J.; Liu, Z. Can Graphene Be Used as a Substrate for Raman Enhancement? Nano Lett. 2009, 10 (2), $553-561$.

5-21. Liu, D.; Chen, X.; Hu, Y.; Sun, T.; Song, Z.; Zheng, Y.; Cao, Y.; Cai, Z.; Cao, M.; Peng, L. Raman Enhancement on Ultra-Clean Graphene Quantum Dots Produced by Quasi-Equilibrium Plasma-Enhanced Chemical Vapor Deposition. Nat. Commun. 2018, 9 (1), 193.

5-22. Bourquard, F.; Tite, T.; Loir, A.-S.; Donnet, C.; Garrelie, F. Control of the Graphite Femtosecond Ablation Plume Kinetics by Temporal Laser Pulse Shaping: Effects on Pulsed Laser Deposition of Diamond-Like Carbon. J. Phys. Chem. C 2014, 118 (8), 4377-4385.

5-23. Perez, D.; Lewis, L. J. Ablation of Solids under Femtosecond Laser Pulses. Phys. Rev. Lett. 2002, 89 (25), 255504.

5-24. Tan, B.; Venkatakrishnan, K. Synthesis of Fibrous Nanoparticle Aggregates by Femtosecond Laser Ablation in Air. Opt. Express 2009, 17 (2), 1064.

5-25. Estrade-Szwarckopf, H. XPS Photoemission in Carbonaceous Materials: A "defect" Peak beside the Graphitic Asymmetric Peak. Carbon 2004, 42 (8-9), 1713-1721.

5-26. Matsuoka, M.; Isotani, S.; Mansano, R. D.; Sucasaire, W.; Pinto, R. A.; Mittani, J. C.; Ogata, K.; Kuratani, N. X-Ray Photoelectron Spectroscopy and Raman Spectroscopy Studies on 
Thin Carbon Nitride Films Deposited by Reactive RF Magnetron Sputtering. World J. Nano Sci. Eng. 2012, 2 (2), 92.

5-27. Hontoria-Lucas, C.; López-Peinado, A. J.; López-González, J. de D.; Rojas-Cervantes, M. L.; Martín-Aranda, R. M. Study of Oxygen-Containing Groups in a Series of Graphite Oxides: Physical and Chemical Characterization. Carbon 1995, 33 (11), 1585-1592.

5-28. Aroca, R. Surface-Enhanced Vibrational Spectroscopy; John Wiley \& Sons, 2006.

5-29. Schatz, G.; Young, M.; Van Duyne, R. Electromagnetic Mechanism of SERS. Surf.Enhanc. Raman Scatt. 2006, 19-45.

5-30. Le Ru, E.; Etchegoin, P. Principles of Surface-Enhanced Raman Spectroscopy: And Related Plasmonic Effects; Elsevier, 2008.

5-31. Persson, B. N. J.; Zhao, K.; Zhang, Z. Chemical Contribution to Surface-Enhanced Raman Scattering. Phys. Rev. Lett. 2006, 96 (20), 207401.

5-32. Otto, A. The "chemical" (Electronic) Contribution to Surface-enhanced Raman Scattering. J. Raman Spectrosc. 2005, 36 (6-7), 497-509.

5-33. Lu, Y. H.; Chen, W.; Feng, Y. P.; He, P. M. Tuning the Electronic Structure of Graphene by an Organic Molecule. J. Phys. Chem. B 2009, 113 (1), 2-5.

5-34. Ling, X.; Fang, W.; Lee, Y.-H.; Araujo, P. T.; Zhang, X.; Rodriguez-Nieva, J. F.; Lin, Y.; Zhang, J.; Kong, J.; Dresselhaus, M. S. Raman Enhancement Effect on Two-Dimensional Layered Materials: Graphene, H-BN and MoS2. Nano Lett. 2014, 14 (6), 3033-3040.

5-35. Powell, J. A.; Venkatakrishnan, K.; Tan, B. Hybridized Enhancement of the SERS Detection of Chemical and Bio-Marker Molecules through Au Nanosphere Ornamentation of Hybrid Amorphous/crystalline Si Nanoweb Nanostructure Biochip Devices. J. Mater. Chem. B 2016, 4 (34), 5713-5728. 
5-36. Santos, P. A.; Rocha, C. S.; Baptista, M. S. Adhesion and Proliferation of HeLa and Fibroblast Cells on Chemically-Modified Gold Surfaces. Colloids Surf. B Biointerfaces 2014, 123, 429-438.

5-37. Hong, Y.; Fan, H.; Zhang, X. Synthesis and Protein Adsorption of Hierarchical Nanoporous Ultrathin Fibers. J. Phys. Chem. B 2009, 113 (17), 5837-5842.

5-38. Lamour, G.; Eftekhari-Bafrooei, A.; Borguet, E.; Souès, S.; Hamraoui, A. Neuronal Adhesion and Differentiation Driven by Nanoscale Surface Free-Energy Gradients. Biomaterials 2010, 31 (14), 3762-3771.

5-39. Kneipp, J. Nanosensors Based on SERS for Applications in Living Cells. Surf.-Enhanc. Raman Scatt. 2006, 335-349.

5-40. Kunzmann, A.; Andersson, B.; Thurnherr, T.; Krug, H.; Scheynius, A.; Fadeel, B. Toxicology of Engineered Nanomaterials: Focus on Biocompatibility, Biodistribution and Biodegradation. Biochim. Biophys. Acta BBA - Gen. Subj. 2011, 1810 (3), 361-373.

5-41. Lesniak, A.; Salvati, A.; Santos-Martinez, M. J.; Radomski, M. W.; Dawson, K. A.; Åberg, C. Nanoparticle Adhesion to the Cell Membrane and Its Effect on Nanoparticle Uptake Efficiency. J. Am. Chem. Soc. 2013, 135 (4), 1438-1444.

5-42. Roiter, Y.; Ornatska, M.; Rammohan, A. R.; Balakrishnan, J.; Heine, D. R.; Minko, S. Interaction of Nanoparticles with Lipid Membrane. Nano Lett. 2008, 8 (3), 941-944.

5-43. Kneipp, J.; Kneipp, H.; McLaughlin, M.; Brown, D.; Kneipp, K. In Vivo Molecular Probing of Cellular Compartments with Gold Nanoparticles and Nanoaggregates. Nano Lett. 2006, 6 (10), 2225-2231. 
5-44. Shukla, R.; Bansal, V.; Chaudhary, M.; Basu, A.; Bhonde, R. R.; Sastry, M. Biocompatibility of Gold Nanoparticles and Their Endocytotic Fate inside the Cellular Compartment: A Microscopic Overview. Langmuir 2005, 21 (23), 10644-10654.

5-45. Chithrani, B. D.; Ghazani, A. A.; Chan, W. C. Determining the Size and Shape Dependence of Gold Nanoparticle Uptake into Mammalian Cells. Nano Lett. 2006, 6 (4), 662-668.

5-46. Dykman, L. A.; Khlebtsov, N. G. Uptake of Engineered Gold Nanoparticles into Mammalian Cells. Chem. Rev. 2014, 114 (2), 1258-1288.

5-47. Zhu, M.; Nie, G.; Meng, H.; Xia, T.; Nel, A.; Zhao, Y. Physicochemical Properties Determine Nanomaterial Cellular Uptake, Transport, and Fate. Acc. Chem. Res. 2013, 46 (3), 622631.

5-48. Ochsenkühn, M. A.; Jess, P. R. T.; Stoquert, H.; Dholakia, K.; Campbell, C. J. Nanoshells for Surface-Enhanced Raman Spectroscopy in Eukaryotic Cells: Cellular Response and Sensor Development. ACS Nano 2009, 3 (11), 3613-3621.

5-49. Movasaghi, Z.; Rehman, S.; Rehman, I. U. Raman Spectroscopy of Biological Tissues. Appl. Spectrosc. Rev. 2007, 42 (5), 493-541. 